University of Louisville

ThinkIR: The University of Louisville's Institutional Repository

Electronic Theses and Dissertations

$5-2013$

\title{
Genetic mapping and mechanism of action of rat mammary carcinoma susceptibility quantitative trait locus Mcs1b.
}

Aaron D. denDekker

University of Louisville

Follow this and additional works at: https://ir.library.louisville.edu/etd

\section{Recommended Citation}

denDekker, Aaron D., "Genetic mapping and mechanism of action of rat mammary carcinoma susceptibility quantitative trait locus Mcs1b." (2013). Electronic Theses and Dissertations. Paper 330. https://doi.org/10.18297/etd/330

This Doctoral Dissertation is brought to you for free and open access by ThinkIR: The University of Louisville's Institutional Repository. It has been accepted for inclusion in Electronic Theses and Dissertations by an authorized administrator of ThinkIR: The University of Louisville's Institutional Repository. This title appears here courtesy of the author, who has retained all other copyrights. For more information, please contact thinkir@louisville.edu. 


\title{
GENETIC MAPPING AND MECHANISM OF ACTION OF RAT MAMMARY
} CARCINOMA SUSCEPTIBILITY QUANTITATIVE TRAIT LOCUS MCSIB

\section{By}

Aaron D. denDekker

B.S. University of Louisville, 2001

M.S. University of Louisville, 2012

\author{
A Dissertation \\ Submitted to the Faculty of the \\ School of Medicine of the University of Louisville \\ in Partial Fulfillment of the Requirements \\ for the Degree of
}

Doctor of Philosophy

Department of Biochemistry and Molecular Biology

University of Louisville

Louisville, KY

May 2013 
Copyright 2013 by Aaron David denDekker

All rights reserved 
GENETIC MAPPING AND MECHANISM OF ACTION OF RAT MAMMARY CARCINOMA SUSCEPTIBILITY QUANTITATIVE TRAIT LOCUS MCS1B

By

Aaron David denDekker

B.S. University of Louisville, 2001

M.S. University of Louisville, 2012

A Dissertation Approved on

January 9, 2013

by the following Dissertation Committee:

David Samuelson, Dissertation Director

Ronald Gregg

Carolyn M. Klinge

David Hein

Russell A. Prough 


\section{DEDICATION}

This dissertation is dedicated to my friend and colleague

Michael Wayne Gordon

who was taken from this world too soon.

Vita mutatur, non tollitur. 


\section{ACKNOWLEDGEMENTS}

I would like to express gratitude to all of the people who helped me along my way, both academically and personally. I would like to thank my mentor, Dr. David Samuelson, for his patience and guidance. As his first graduate student there were some growing pains for us both, but his door was always open and I've learned so much from him over the past few years. I want to thank my dissertation committee, Drs. Carolyn Klinge, Russell Prough, Ronald Gregg, and David Hein, for their support and assistance. I would like to extend a special thanks to Ron, as well as Dr. Jill Suttles, for giving me the opportunity to work in their respective labs prior to entering graduate school. The skills and lessons I learned from them helped prepare me for what was to come. I would like to thank the department of Biochemistry and Molecular Biology and its faculty for affording me the opportunity to pursue this degree. I would like to especially thank Drs. William Dean, Steven Ellis, and Barbara Clark for their friendship and stewardship through the years. You were all also such excellent teachers and had a huge impact on my growth and development as a scientist as well as a person.

I want to thank Joe Moore for his friendship and support. Without him graduate school would not have been the same. I want to thank Traci Kruer for being my veep, helping me format my dissertation, and just listening and being a good friend... I really appreciate everything you've done for me. I want to thank Alan Kerr and Huy Le for their thought-provoking and informed discussions and for just being great friends. I want to give special recognition to Mike Gordon. Mike, you were so bright and such a good 
person and you had such potential to do great things in the world. I am lucky to have had you as such a close friend and confidant. I miss you. You will always live in our hearts. I would also like to thank all of my other fellow graduate students, former and current, for their support and willingness to listen to my presentations.

Last, but not least, I want to thank my lovely wife, Tara, for her unwavering support and encouragement through everything. She was working full-time and working on her own graduate degree in nursing, but still found the strength and the words to help keep me going when I didn't think I could go any further. I love her and my wonderful children, Paul and Bowie, more than I can express. I could not have done any of this without all of them. They make it all so much more worth it. 


\title{
ABSTRACT \\ GENETIC MAPPING AND MECHANISM OF ACTION OF RAT MAMMARY CARCINOMA SUSCEPTIBILITY QUANTITATIVE TRAIT LOCUS $M C S 1 B$
}

\author{
Aaron D. denDekker
}

January 9,2013

Breast cancer is a complex disease that involves genetic, epigenetic, and environmental components. High and moderate penetrant genes have been identified that affect risk to developing breast cancer; however, these risk alleles are present in a small percentage of breast cancer cases. Low penetrant modifier genes have risk-associated alleles that are common in the population. Although these genes have lower penetrance, it is expected that the majority of genetic risk to developing breast cancer is controlled by common genetic variation. Studying mechanisms of common genetic variants on breast cancer risk is difficult due to their small individual effects and overlapping contribution of other risk factors; thus, animal models are commonly used. The rat mammary carcinoma susceptibility quantitative trait locus (QTL) Mcs $1 b$ was identified between mammary carcinoma-resistant Copenhagen (COP) and susceptible Wistar Furth (WF) rats on chromosome 2. This rat QTL is an ortholog of a human breast cancer-associated locus identified on human chromosome $5 q$; therefore, the rat $M c s 1 b$ model can be used to identify mechanisms and causative factors contributing to breast cancer risk associated with human breast cancer-associated locus $5 q$. 
The goal of the work presented in this dissertation is to identify quality candidate breast cancer risk genetic elements associated with the rat $M c s l b$ locus. This project utilized a well-defined rat mammary carcinogenesis system and congenic rat model to fine map and characterize the rat $M c s l b$ locus. My studies reduced the number of candidate genes by narrowing the rat $M c s 1 b$ locus from a 13 megabase (Mb) to a $1 \mathrm{Mb}$ containing nine annotated transcripts. I determined that $M c s I b$-conferred mammary carcinoma resistance is being controlled by a cell type within the mammary gland. This is an important finding because mammary carcinogenesis is dependent on both mammary gland-extrinsic and -intrinsic factors. I also found that the transcript Mier3 is differentially expressed between resistant and susceptible rat mammary glands with or without carcinogen exposure providing genetic evidence that Mier 3 is a strong mammary carcinoma susceptibility gene. Taken together, these results provide insight into the mechanism by which Mier3 controls mammary carcinogenesis and implicate human MIER3 as a potential target for breast cancer prevention. 


\section{TABLE OF CONTENTS}

\section{PAGE}

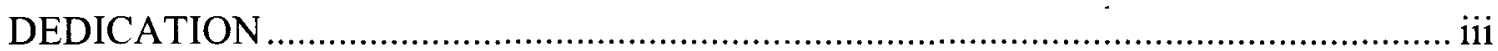

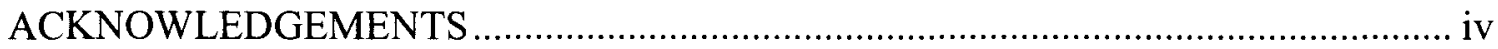

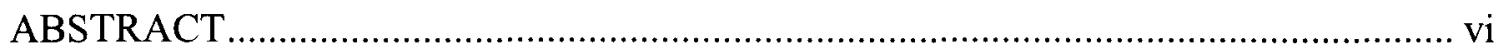

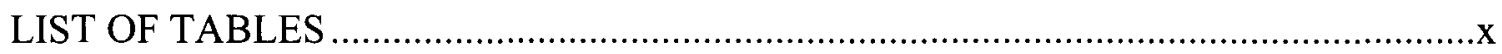

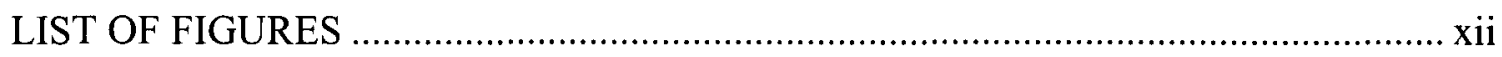

\section{CHAPTER}

I. GENES, ENVIRONMENT, AND BREAST CANCER

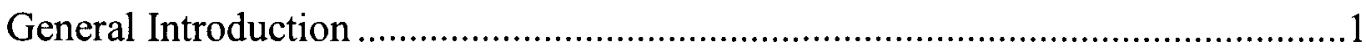

Breast Cancer Risk Factors: Breast Cancer as a Complex Disease ............................5

Animal Models of Breast Cancer Susceptibility....................................................24

Mammary Carcinoma Susceptibility Quantitative Trait Loci ................................32

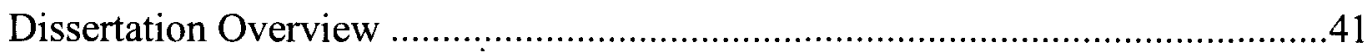

II. RAT MCSIB IS WITHIN A 1 MB REGION OF RAT CHROMOSOME $2 \ldots \ldots \ldots . . .43$

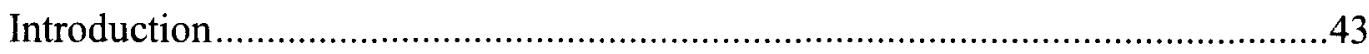

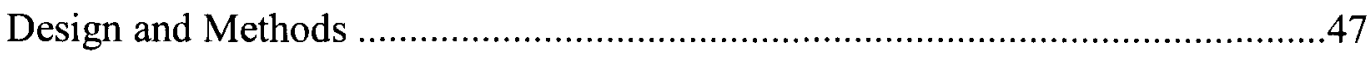

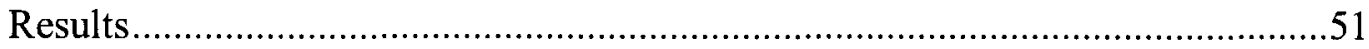

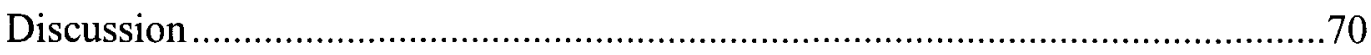

III. RAT MCSIB ACTS IN A MAMMARY GLAND CELL-AUTONOMOUS MANNER TO CONTROL MAMMARY CARCINOMA SUSCEPTIBILITY .......82

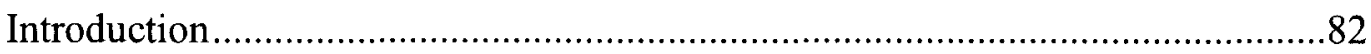

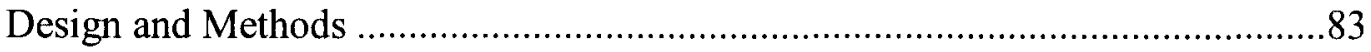




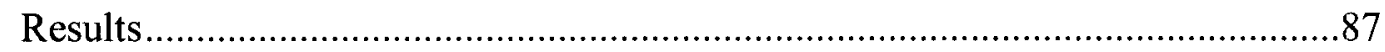

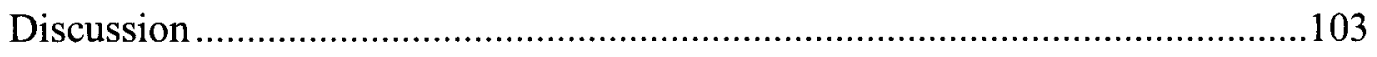

IV. MIER3 IS A CANDIDATE BREAST CANCER-ASSOCIATED GENE .............110

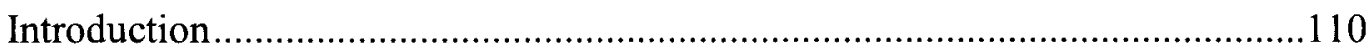

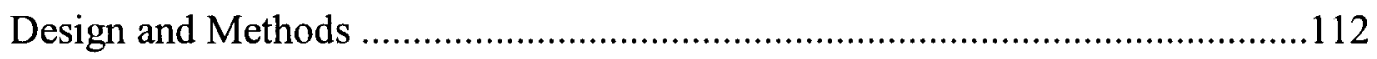

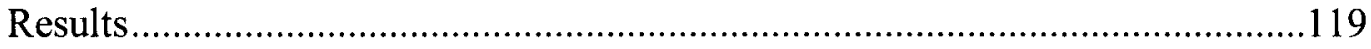

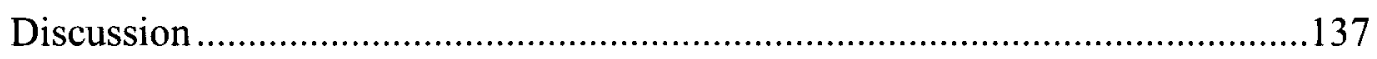

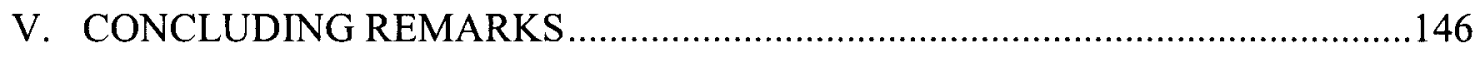

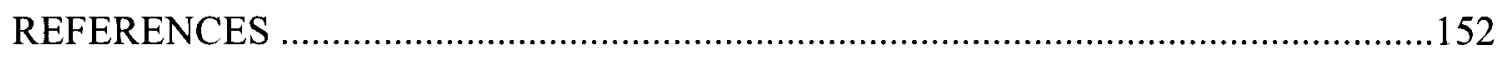

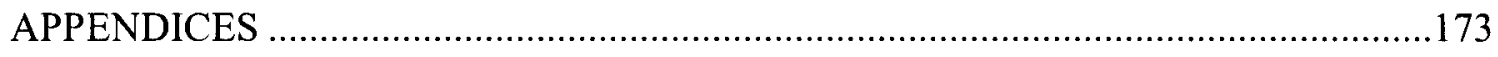

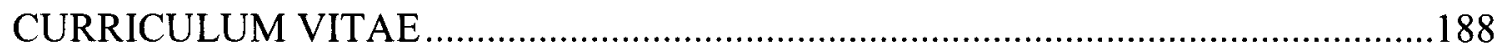




\section{TABLE}

\section{LIST OF TABLES}

1. Informative microsatellite markers used to narrow $M c s 1 b$ locus to $1.8 \mathrm{Mb} \ldots \ldots . .50$.

2. Mammary carcinoma multiplicity phenotypes (mean mammary carcinomas per rat \pm SD) by genotype for WF.COP Chr 2 congenic lines used to map Mcslb to 1 $\mathrm{Mb}$

3. Primer Sequences for Microsatellites in $0.66 \mathrm{Mb}$ region of $M c s 1 b$ locus ..............57

4. SNP markers tested on WF and COP rats based on published data of SNPs in the $M c s 1 b$ interval. 58

5. Regions sequenced between $\mathrm{Rat} \mathrm{Chr} 2: 43125940$ and $\mathrm{Chr} 2: 43632545$ to identify potential polymorphisms between WF and COP in the Mcslb locus. 64

6. Graft site MG development outcome (dependent) and Mcs $1 b$ donor and recipient genotypes (independent)

7. MG graft site tumor outcome (dependent) and $M c s l b$ donor and recipient genotypes (independent)

8. Mammary gland outcome in enzymatically-dissociated versus mechanicallydissociated mammary gland cell preparations

9. Primers for resequencing ORFs and 3 ' untranslated regions of transcripts in 1.8 $\mathrm{Mb} M c s 1 b$ region 114

10. Rat Mcs $1 b$ Target and Rplp2 Primers and Probe Sequences Used for QPCR.....117 
11. BLAT alignment of ENSRNOG00000034909 sequence against UCSC Genome Browser Rat DNA build (Baylor 3.4/rn4 assembly) ........................................123

12. Analysis and statistics of $M c s I b$ potential candidate gene mammary gland transcript levels in Mcs1b-resistant and susceptible genotypes at 12 weeks of age

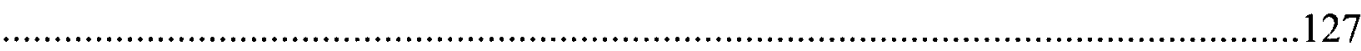

13. Primer and Probe Sequences Used for Analysis of Rat Mier3 Variants. 133 


\section{LIST OF FIGURES}

\section{FIGURE}

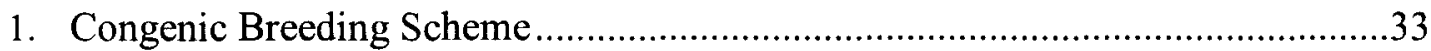

2. Chromosome 2 genetic map of $M c s l$-congenic and -recombinant rat lines..........37

3. $280 \mathrm{~Kb}$ haplotype block containing breast cancer-associated SNP $r s 889312 \ldots \ldots . . .39$

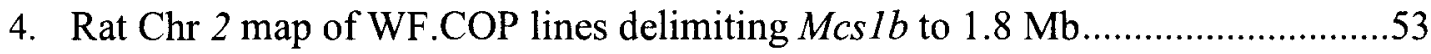

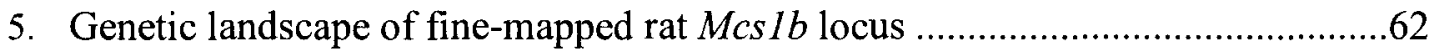

6. Endogenous mammary tumors formed in congenic line $1 b-11$ and homozygous WF animals .66

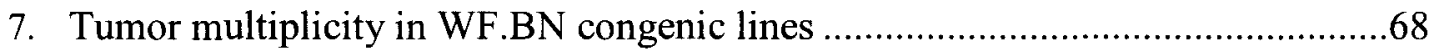

8. Map of human $M c s 1 b$ orthologous locus on human chromosome 5 .................... 71

9. Additional breast cancer-associated SNPs identified in GWAS with rat

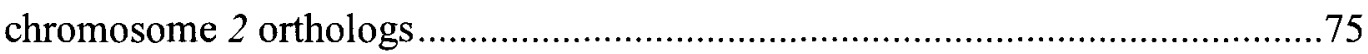

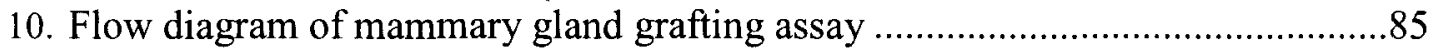

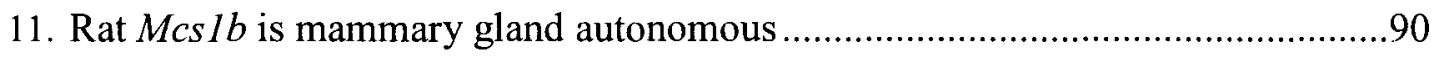

12. Representative whole mounted interscapular fat pad from MEC pilot study ........95

13. Tumor outcome in ectopic mammary glands derived from MECs.....................97

14. Tumor outcome in ectopic mammary glands derived from MECs or MECs

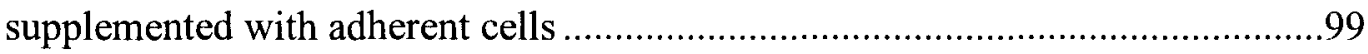

15. Results of DMBA susceptibility window studies with MEC injections..............102

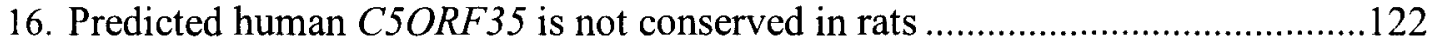


17. Mier 3 is not differentially expressed between WF susceptible and Mcs $1 b$ resistant WF.COP congenic females in rat ovary, brain, spleen, or thymus tissues 129

18. Three different Mier 3 variants are expressed in the rat mammary gland 131

19. Rat Mcslb-resistant genotype is associated with higher body weight 136

20. Rat Mier3 transcript levels were significantly higher in DMBA-induced mammary carcinomas compared to non-diseased mammary gland tissue and human MIER3 was significantly higher in breast carcinomas compared with pathologically normal breast tissues 139 


\section{CHAPTER I}

\section{GENES, ENVIRONMENT, AND BREAST CANCER}

\section{General Introduction}

\section{Breast Cancer Statistics}

Breast cancer is the leading cause of cancer death in the United States in women aged 20-59 and is the second most diagnosed cancer in women in the US (Siegel et al., 2012). Additionally, with the exception to skin cancer, breast cancer is the most common cancer diagnosed in women worldwide (Mathers et al., 2008). In the US, it was estimated that near 230,000 new cases of invasive breast cancer were diagnosed and that almost 40,000 women died of breast cancer in 2012 (American Cancer Society, 2011). Breast cancer deaths in women rose $0.4 \%$ per year from 1975 to 1990 . However, in recent years, early detection, increased awareness, better treatments, and decreased use of hormone replacement therapies have led to a decrease in breast cancer death rates with a 2.2\% decrease per year from 1990 to 2007 (American Cancer Society, 2011). Still, breast cancer remains a major health concern for many women and current risk estimates are that 1 out of 8 women in the US will develop breast cancer (Altekruse SF, 2010).

Breast cancer also affects men; however, male breast cancer only accounts for approximately $1 \%$ of breast cancers diagnosed in the US (American Cancer Society, 2011). Due to its rarity, much less is known about the male form of breast cancer. Incidence of male breast cancer has risen from 1 in 100,000 men being diagnosed with 
breast cancer in the late 1970 's to 1.2 in 100,000 from 2000-2004 (Onami et al., 2010). However, breast cancer death rates in men have fallen $3.3 \%$ since 2000 ; likely due to increased awareness and better diagnoses (American Cancer Society, 2011; Anderson and Devesa, 2005).

\section{Breast Cancer Risk}

Breast cancer is a complex disease and the risk of developing it has environmental, genetic, and epigenetic components. The most important factors affecting risk to developing breast cancer are age and female gender (American Cancer Society, 2011). As noted, approximately $12 \%$ ( 1 in 8 ) of US women are predicted to be diagnosed with breast cancer based on lifetime risk, and this probability increases as a woman gets older (Altekruse SF, 2010; American Cancer Society, 2011). This estimate is based on epidemiology of population incidence. However, these estimates may not be accurate for many individual women. Risk for an individual may be higher or lower depending on various risk factors including age, family history and reproductive history as well as other heritable and non-heritable factors (Gail et al., 1989).

Radiation exposure is one of the most potent exogenous factors known to increase chances for developing breast cancer (Land et al., 2003; Ronckers et al., 2005). Although exposure to high doses of ionizing radiation is less common, many women are exposed to low-dose radiation through mammograms and routine low-dose exposure has been associated with an increase in breast cancer risk especially in women already with an increased familial risk (Pijpe et al., 2012). Additionally, other non-heritable risk factors have been identified e.g., living near nuclear power sites (chronic radiation exposure) and 
shift work cycles (prolonged exposure to light at night) but these risks aren't fully understood (Boice et al., 2003; Bonde et al., 2012; Hill et al., 2011).

Inherited genetic susceptibility to cancer is now considered an established fact (Fletcher and Houlston, 2010). Mutations in several high-risk genes have been identified, e.g. $B R C A 1$ and $B R C A 2$, that increase a woman's chances of developing breast cancer by $51-75 \%$ and 33-54\%, respectively (Antoniou et al., 2003). Screening for mutations in these genes is, under certain circumstances, more routinely conducted; however, mutations in these genes account for approximately $3-5 \%$ of female breast cancer cases, leaving the majority of alleles contributing to breast cancer susceptibility unknown (Campeau et al., 2008; Narod and Salmena, 2011). Conversely, common genetic modifiers have a small independent effect but can act cumulatively to exert a greater influence on disease development (Jostins and Barrett, 2011; Pharoah et al., 2008). Therefore, the majority of genetic risk to breast cancer development is likely attributable to common genetic variation (Fletcher and Houlston, 2010; Lee et al., 2011).

\section{Predicting Breast Cancer Risk}

Accurate prediction of an individual's breast cancer risk is vital to developing better prevention and treatment strategies. The ability to predict the development of disease in an individual has been useful in improving strategies for breast cancer prevention; e.g. increased frequency of mammograms for high risk breast cancer patients (Jostins and Barrett, 2011). However, breast cancer etiology is complex, which makes accurate individual risk assessment problematic for many women. Individual risk for developing breast cancer is currently assessed using the Gail model. Gail and colleagues 
developed a risk-assessment model based on a set of risk factors: age at menarche, age at first live birth, number of previous biopsies, and number of first-degree relatives with breast cancer (Costantino et al., 1999; Gail et al., 1989). An interactive tool has been developed by the National Cancer Institute (NCI) and the National Surgical Adjuvant Breast and Bowel Project (NSABP) based on the Gail model. This tool is used by physicians to assess risk for an individual with a limited family history (http://www.cancer.gov/bcrisktool/).

The Gail model provides an efficient method to project the likelihood to develop breast cancer for most individual women; however, it has limitations. First, the Gail model only accounts for first degree relatives with breast cancer potentially underestimating risk in the $50 \%$ of families with cancer in the paternal lineage (Euhus et al., 2002; Evans and Howell, 2007). Also, the Gail model does not take into account the age of onset of breast cancer of the affected relative, thereby, possibly overestimating risk in women with an affected relative who developed breast cancer late in life. Last, although it has been modified to account for ethnic background, the Gail model focuses primarily on non-genetic risk factors. Heritable factors are recognized to have significant roles in complex disease risk and this is underscored by the importance of accounting for family history in risk assessment (Bevier et al., 2011; Costantino et al., 1999; Gail et al., 1989; Lalloo and Evans, 2012).

At present, the effects of risk-predisposing genes are difficult to evaluate; thus, a better understanding of genetics and the molecular mechanisms that influence breast cancer susceptibility is necessary to better predict an individual's risk for developing breast cancer. Several low- to moderate-penetrance breast cancer risk alleles have been 
identified (Easton et al., 2007; Turnbull et al., 2010). Relative risk conferred by alleles at individual loci is small, but risk alleles are hypothesized to act multiplicatively. It has been estimated that risk of developing breast cancer is approximately six times as great among women carrying 14 risk alleles as among those carrying no risk alleles at these loci (Pharoah et al., 2008). Although there is little clinical use for single, low-penetrance genes, the cumulative effects of these alleles may be useful to separate high risk

individual women from those at lower risk (Pharoah et al., 2008). As previously mentioned, the genetic contribution to breast cancer development is not fully understood. However, new technologies and approaches are available that will allow for the discovery of common genetic risk alleles and it is believed that assessing individual breast cancer risk based on genetic factors will be achieved in the near future (Jostins and Barrett, $2011)$.

\section{Breast Cancer Risk Factors: Breast Cancer as a Complex Disease}

\section{Estrogen Exposure and Breast Cancer}

As stated earlier, the most widely recognized risk factors to breast cancer development are female gender and increasing age (American Cancer Society, 2011). Women develop breast cancer at a rate one hundred times that of men and these rates increase as women age. This is interpreted as a representation of accumulated exposure to ovarian hormones in the form of estrogens and progesterone (Pike et al., 1993). It is thought that women exposed longer to estrogen due to early menarche, late menopause or hormone replacement therapy exhibit an increase in breast cancer risk (Kelsey et al., 1993; Pike et al., 1993). It was first discovered in 1896 that oophrectomy could 
effectively regress breast carcinomas (Beatson, 1896). Later observations indicated that women who experienced early menarche or late menopause exhibited higher incidence of breast cancer (Kelsey et al., 1993). Also, women who had their first child early (before age 18) had a lower incidence of breast cancer than women who had children later in life (35 years of age) (Kelsey et al., 1993; MacMahon et al., 1970; Pike et al., 1983). More recently, it has been shown that oophrectomy in women before 35 years of age reduces breast cancer risk by $75 \%$ (Kronenberg and Williams, 2008). Taken together, this suggests a pivotal role for endogenous estrogens in the development of breast cancer.

Estrogen exposure is now considered one of the most important factors determining breast cancer risk. Estrogens primarily function to control the estrous cycle but have roles in a variety of other processes. There are three forms of estrogen produced in the body: estrone (E1), estradiol (E2), and estriol (E3). These are made and secreted primarily by the ovary but can also be made in other tissues. Of these E2 is the most potent as $E 1$ and $E 3$ bind estrogen receptors $\alpha$ and $\beta(E R \alpha$ and $E R \beta)$ with lower affinity compared to E2. However, E1 and E3 can be converted to E2. Significantly, E2 is secreted by the breast epithelium and adipose and serum E2 levels have been correlated with an increase in breast cancer incidence (Kronenberg and Williams, 2008). During the 1940s, hormone replacement therapy (HRT) was instituted into clinical practice for the treatment of menopausal symptoms by administration of exogenous estrogens. Subsequent studies of groups of women receiving HRT demonstrated that the relative risk (RR) of developing breast cancer for women receiving therapy was elevated $(R R=2.0)$ compared to that of the general population $(R R=1.3)$ (Hoover et al., 1976). Breast cancer risk due to HRT was debated for many years citing contradictory studies; 
however, studies conducted by the Women's Health Initiative (WHI) and the Million Women Study (MWS) indicate that continued HRT increases breast cancer risk (Beral, 2003; Rossouw et al., 2002). Data from the MWS showed the effect of combined progesterone-estrogen therapy was greater $(\mathrm{RR}=2.00,[95 \%$ Confidence Interval $(\mathrm{CI})$ 1.88-2.12], $\mathrm{P}<0.0001)$ compared to estrogen alone (1.30 [1.21-1.40], $\mathrm{P}<0.0001)$ when compared to women never receiving HRT (Beral, 2003). Women in the WHI randomized control study receiving combined estrogen-progesterone therapy also had an increase incidence in breast cancer compared to those receiving a placebo (Hazard Ratio $(\mathrm{HR})=1.62[95 \%$ CI 1.00-1.59], $\mathrm{P}<0.05)$ (Rossouw et al., 2002).

Estrogens have been extensively studied to understand the action by which they influence breast cancer. Estrogens promote rapid proliferation of mammary epithelial cells, which increases the probability that mutations will become fixed and propagated, thereby promoting tumor formation (Preston-Martin et al., 1993). The "canonical" estrogen signaling pathway occurs through estrogens binding ER $\alpha$ and ER $\beta$. Estrogens diffuse passively through cell and nuclear membranes and bind to $\operatorname{ER} \alpha$ and $\operatorname{ER} \beta$ (Kronenberg and Williams, 2008). Once an estrogen receptor is bound to an estrogen ligand, it undergoes a conformational change and binds to specific DNA sequences called estrogen response elements (EREs) to drive transcription of target genes. ERE-bound ERs interact with basal transcription factors and co-activator proteins, which stabilize basal transcription factor binding and initiate transcription (Klinge, 2000). The products of these genes act to promote cell growth and differentiation.

$\mathrm{ER} \alpha$ and $\mathrm{ER} \beta$ exhibit similar binding affinities for E2, the predominate estrogen in premenopausal women, and both ER subtypes have been shown to bind to EREs 
similarly (Kuiper et al., 1997; Paech et al., 1997). Both receptors form homodimers in response to ligand binding; however, studies have shown that they can also form heterodimers in vitro and in vivo, and that $\mathrm{ER} \beta$ reduces $\mathrm{ER} \alpha$ transcriptional activity (Hall and McDonnell, 1999; Pettersson et al., 2000; Pettersson et al., 1997). Also, recent findings have shown that $\operatorname{ER} \beta$ can recruit a corepressor complex to the ER $\alpha$ gene promoter resulting in reduced expression of $E R \alpha$ (Bartella et al., 2012). This implies that $\mathrm{ER} \beta$ is acting as an internal regulator of $\mathrm{ER} \alpha$ activity. Indeed, $\mathrm{ER} \alpha$ expression is often higher compared to ER $\beta$ in invasive mammary tumors (Leygue et al., 1998).

ERs are the active components controlling estrogen signaling and have, thus, become popular targets for breast cancer treatment. There are several classes of hormone treatment options for breast cancer therapy. Selective estrogen-receptor modulators (SERMs), such as tamoxifen and raloxifene, are synthetic agonist/antagonists of the ER. In the mammary gland, both of these drugs bind ER as antagonists to prevent ERmediated transcription. Alternatively, tamoxifen is an ER agonist in uterus and bone while raloxifene acts as an agonist in bone only (Dutertre and Smith, 2000). Other chemotherapeutics also act on the ER, such as fulvestrant, an ER antagonist that abolishes estrogen-specific gene transcription by degrading the ER (Flemming et al., 2009). Aromatase inhibitors (AI), on the other hand, operate indirectly by inhibiting the activity of aromatase, the enzyme responsible for converting androgens into estrogens, thereby reducing estrogen availability for ER binding (Mokbel, 2002).

Tamoxifen is the most widely used treatment for ER-positive breast cancers in pre- and post-menopausal women. Although tamoxifen is an effective therapeutic, it has some disadvantages and limitations. For one, tamoxifen has been shown to increase the 
chance of endometrial cancer by acting as an agonist in the uterus and endometrium (Fisher et al., 1994; Gottardis et al., 1988). Also, many breast tumors are unresponsive to tamoxifen as they have lost the ability to express ER $\alpha$ (Ring and Dowsett, 2004). One of the biggest problems clinicians face when treating with tamoxifen is that many patients develop resistance to the drug and relapse (Cui et al., 2012; Osborne et al., 2005). Often, tumors in these patients continue to express a functionally normal ER yet they grow independently of estrogen action. Amplification and over-expression of the growth factor receptor HER2 is thought to be a major mechanism contributing to endocrine resistance in many cases (Osborne et al., 2005). Additionally, it is believed that tamoxifen resistance in some breast cancers may is due to changes in expression of co-activators and co-repressors (Dobrzycka et al., 2003). For example, the co-repressor Metastatic tumor antigen 1 (MTAl) interacts directly with ER and histone deacetylases (HDACs) to inhibit ERE transcriptional activity and promote hormone-independent growth. Further, MTA1 over-expression correlates with a reduced response to tamoxifen (O'Malley and Kumar, 2009). In addition, mis-expression of many of these co-regulators is associated . with many cancer types. It is not clear whether this dysregulation is a cause or consequence of the pathology; however, co-regulators of ER action appear to be important factors in breast cancer pathogenesis.

Estrogens also function to promote breast carcinogenesis independent of the canonical ER-signaling pathway. Several mechanisms have been identified that are independent of estrogen receptor activity. For example, Liehr et al. have shown that 4hydroxylated estrogen metabolites play a central role in the genotoxic activity of estrogen via generation of free radicals, which correlates with increased cancer risk (Liehr, 1990; 
Liehr and Roy, 1990). Second, estrogen metabolites, catechol estrogen quinones, are formed via cytochrome P450 activation. These electrophilic quinine metabolites form depurinating adducts on DNA thereby inducing point mutations (Cavalieri et al., 2002). Normally, metabolism of these catechol estrogens by $O$-methylation, glutathionation, glucuronidation, or sulfation renders these inactive; however, studies have shown that when levels of catechol estrogen metabolites are increased they cannot be sufficiently metabolically inactivated, and this associates with an increase in breast cancer incidence (Rogan et al., 2003). In addition, Barrett and colleagues demonstrated that diethylstilbestrol (DES), a synthetic estrogen previously used for the treatment of menopause, interferes with microtubule organization at low doses (Tsutsui et al., 1983). Their data indicate that DES does not prevent cell division but, rather, interferes with microtubule organization sufficiently to cause non-dysjunction at mitosis resulting in aneuploidy (Tsutsui et al., 1983). Therefore, endogenous estrogens may also be affecting carcinogenesis through this pathway.

Various genetic modifiers have been identified that affect estrogen action in the context of breast cancer susceptibility. Common variants have been identified in, ESR2, the gene encoding ER $\beta$ (Maguire et al., 2005). Gene targeting association studies along with in vitro studies suggest these variants may be involved in increasing risk for breast cancer. Interestingly, an ultra-rapid metabolizing allele for $C Y P 2 C 19, C Y P 2 C 19 * 17$, has been identified that associates with a lower risk for breast cancer (Justenhoven et al, 2009). Additionally, deletion alleles of CYP2C19, were identified at an increased frequency in familial breast cancer cases from a cohort of Northern Finnish women (Pylkas et al., 2012). CYP2C19 is a gene encoding an enzyme involved in the catabolism 
of E2 implying that increased catabolism of E2 reduces estrogen levels and subsequently reduces breast cancer risk. Correspondingly, haploinsufficiency of $C Y P 2 C 19$ may retard estrogen catabolism and, thereby, increase risk for breast cancer. What is more, copy number variants (CNVs) have recently been discovered in familial breast cancer cases that affect genes in estrogen signaling pathways (Pylkas et al., 2012). Taken together, the effects of estrogen signaling in breast cancer development are very complex. Unraveling these mechanisms will be useful in determining the role of estrogens in breast cancer and may lead to better diagnoses and treatments.

\section{Progesterone and Breast Cancer}

Progesterone is another steroid hormone involved in mammary gland development (Kronenberg and Williams, 2008). Like estrogens, progesterone passively diffuses into the cell and binds to either of its cognate receptors, A or B (PR-A or PR-B, respectively) and is able to activate gene transcription (Kronenberg and Williams, 2008). It is believed that estrogens and progesterone act in concert to promote ductal branching and development of mammary terminal end buds (TEBs), while estrogen is responsible for overall growth of mammary ducts emanating from the nipple (Ruan et al., 2005; Singletary and McNary, 1992). Although progesterone is associated with mammary duct outgrowth, studies have also shown that PR-deficient animals develop normal mammary glands (Kleinberg et al., 1990; Lydon et al., 1995). This suggests that progesterone may be involved in ductal morphogenesis by acting through an alternative mechanism. Although the mechanism by which progesterone is acting is not fully understood, it is 
held that progesterone plays a role in promoting mammary gland development (Lydon et al., 1995; Ruan et al., 2005).

Progesterone is also known to attenuate estrogen action via three separate mechanisms (Kronenberg and Williams, 2008). First, progesterone can reduce synthesis of ER $\alpha$ (Hsueh et al., 1976; Tseng and Gurpide, 1975). Second, progesterone can activate transcription of the gene coding for $17 \beta$-hydroxysteroid dehydrogenase (17 $\beta$ HSD), an enzyme involved in the enzymatic inactivation of E2 to the weakly estrogenic E1 (Casey et al., 1994). Third, progesterone induces transcription of estrogen sulfotransferases; enzymes that catalyze the conjugation of sulfate groups to E2 and E1 rendering the estrogens inactive (Falany and Falany, 1996). Therefore, although progesterone promotes mammary gland development, it could play a role in reducing the deleterious effects of estrogen action.

Based on these observations, one may expect progesterone to reduce the protumorigenic effects of estrogen. Not surprisingly, breast tumors that express ER and PR are much more sensitive to endocrine chemotherapies compared to ER- and PR-negative tumors (American Cancer Society, 2011). However, the role of progesterone in breast cancer risk is confounding. The WHI and the MWS Study found that women taking estrogen plus progestin replacement therapy exhibited higher breast cancer incidence than those taking estrogen alone (Chlebowski et al., 2010; Chlebowski et al., 2003; Travis et al., 2010). Additionally, postmenopausal women with prior hysterectomy that received equine conjugated estrogen alone had a lower incidence of breast cancer compared to the placebo group (Anderson et al., 2012). This implicates progesterone with a role in promoting breast cancer development. 
The mechanism by which progesterone and PR affect mammary cell proliferation and cancer development is not fully understood. In vitro studies have shown that progesterone induces cell proliferation by activating protein kinases such as MAPK, Akt/PI3K, and c-Src (Boonyaratanakornkit et al., 2001; Migliaccio et al., 1998; Saitoh et al., 2005); however, the role this plays in human (i.e. whole animal) physiology is not clear and further studies are needed to determine how it effects mammary carcinogenesis. What is more, $\mathrm{PR}$ expression is driven by $\mathrm{ER} \alpha$ but can also be expressed independently of ER $\alpha$ (Horwitz et al., 1982; Lange, 2008). Therefore, it is difficult to ascertain the effects of progesterone alone from those of estrogen (Lange, 2008). Regardless, while the distinct role of progesterone and PR in breast carcinogenesis is unclear, breast tumor PR status is routinely used as a prognostic indicator for response to adjuvant chemotherapy and PR-targeted therapies are in use to treat breast cancer (Lange, 2008).

\section{Exogenous Environmental Effects on Breast Cancer Development}

As described, breast cancer is influenced by both exogenous and endogenous environmental chemical carcinogens. The prime example of a physical carcinogen is irradiation. Less common than estrogen exposure, ionizing radiation is the strongest known exogenous environmental factor affecting breast cancer development (Ronckers et al., 2005). This is based on breast cancer incidence rates in atomic bomb survivors and patients receiving prolonged radiotherapy (Land et al., 2003; Ronckers et al., 2005). The effects of living near nuclear power plants and exposure to low-level ionizing radiation sources remains a concern, but has not been extensively studied (Boice et al., 2003). Unrelated to radiation, there has been increasing interest in "light at night" as a risk factor 
since exposure to light at night due to night shift work correlates with both disruption in melatonin secretion and increased breast cancer incidence (Bonde et al., 2012; Hill et al., 2011). Also, exposure to radiofrequency/microwave radiation emissions (RF) from wireless communications has become a concern due to the increasing use of mobile communication devices. This is thought to play a role in numerous pathologies including breast cancer development; however, these areas are relatively new and are not fully understood (Hardell and Sage, 2008).

Many different classes of exogenous chemical carcinogens have been identified that associate with breast cancer risk. Some of these act though the estrogen signaling pathway. Xenoestrogens, such as polychlorinated biphenyls (PCBs), bisphenol A (BPA) and phthalates are structurally different from endogenous estrogens but are able to bind ERs and activate mitotic events (Darbre and Charles, 2010; Fernandez and Russo, 2010; Kester et al., 2000; Tsutsui et al., 2000). Phytoestrogens, such as genestein, are natural plant-derived xenostrogens. They are structurally similar to endogenous mammalian estrogens and are able to act in a similar manner through the canonical signaling pathway (Martin et al., 1978). Yet, reported effects on phytoestrogens on breast cancer risk are conflicting due to studies that have shown protective as well as detrimental effects (Darbre and Charles, 2010; Martin et al., 1978; Safe, 1997; Zhang and Chen, 2011).

Other chemical carcinogens are able to act to promote breast cancer development through estrogen-independent means. Some of the most prominent are polycyclic aromatic hydrocarbons (PAHs) and their nitrated metabolites (nitro-PAHs), which cause genotoxic effects (Mersch-Sundermann et al., 1993). For example, PAHs such as benzo(a)pyrene (B[a]P) and 7,12-dimethylbenz(a)anthracene (DMBA) are metabolized to 
form reactive diol epoxide intermediates that directly bind DNA and induce mutations (Todorovic et al., 1997). B[a]P is one of the most well-studied PAHs and it is found in many places because it is a byproduct of incomplete combustion of organic matter, e.g., cigarette smoke, car exhaust, and industrial smoke stack exhaust (Lawther and Waller, 1976; Phillips, 1999). DMBA is a synthetic organ-specific PAH also used as a laboratory carcinogen (Christou et al., 1995; Miyata et al., 2001). DMBA induces expression of CYPIBI in a mammary gland-specific manner (Christou et al., 1987). Thus, CYP1B1, is the primary P450 enzyme metabolizing DMBA in the mammary gland. CYP1B1, along with epoxide hydrolase, converts DMBA to its carcinogenic diol-epoxide form, which makes it ideal to induce carcinogenesis in the mammary gland (Christou et al., 1987; Christou et al., 1995; Miyata et al., 1999). DMBA is routinely used as an experimental mutagen for research purposes because it models PAH-induced carcinogenesis (Modi et al., 2012).

\section{Lifestyle-based Factors and Breast Cancer Risk}

Many different lifestyle-based environmental factors are also contributors to breast cancer risk. Tobacco smoke has long been accepted as modifiable lifestyle risk factor for many different cancer types although there is limited evidence for its role in breast cancer (American Cancer Society, 2012). Obesity and alcohol consumption are two other factors that have been identified as risk predictors for developing breast cancer (American Cancer Society, 2011; Carmichael, 2006; Hankinson et al., 1995; Pelucchi et al., 2011). 
In post-menopausal women, obesity is positively associated with an increased incidence of breast cancer and has also been shown to confer a poorer prognosis (Carmichael, 2006; Hankinson et al., 1995). However, studies on pre-menopausal women have had conflicting results as some data suggest an inverse relationship between weight and breast cancer outcome. Regardless, weight gain and obesity associate with poor outcomes in both pre- and post-menopausal women (Carmichael, 2006). Adipocytes exhibit increased aromatase expression, the enzyme responsible for converting androgens to estrogen (Bulun et al., 2012; Santen et al., 2009). Thus, it has been postulated that increased estrogen is produced in the breast adipose tissue thereby promoting breast cancer development (Bulun et al., 2012).

Alcohol consumption increases endogenous estrogen levels and positively correlates with breast density and breast cancer incidence (Boyd et al., 1995; Hankinson et al., 1995). Although alcohol consumption associates with increased incidence of breast and other types of cancers, there are also data that suggest beneficial effects from alcohol, especially in red wine (de Lorimier, 2000). However, the mechanisms for this have not been extensively studied and are currently inconclusive.

Additionally, the consumption of "well-done" or charred meat has been identified as a risk factor to a variety of cancers, including the breast (Zheng and Lee, 2009). Heterocyclic amines (HCAs) are the most abundant mutagen found in overcooked meat. Studies have identified associations between high-temperature cooked meat intake, HCA exposure and breast cancer incidence (De Stefani et al., 1997; Sinha et al., 2000; Zheng et al., 1998; Zheng and Lee, 2009). Women who consistently eat overcooked meat have a 4.6-fold higher $\mathrm{RR}(95 \% \mathrm{CI}=1.36-15.70)$ of developing breast cancer compared to 
women who consume rare or medium-done meat. (Zheng et al., 1998). Further studies found increased levels of 2-amino-1-methyl-6-phenylimidazo(4,5-b)pyridine (PhIP), the most common HCA found in over-cooked meat, to correlate with an increase in breast cancer incidence; however, there was no significant effect associated with other HCAs (Sinha et al., 2000). In addition to HCAs, overcooked meat may also contain other mutagens such as PAHs including the ubiquitous $\mathrm{B}[\mathrm{a}] \mathrm{P}$ (Zheng and Lee, 2009). Other studies suggest that breast cancer risk associated with HCA exposure may be modified by common polymorphisms in genes coding for enzymes responsible for metabolizing HCAs, thereby strengthening the role of genetics in breast cancer risk (Deitz et al., 2000; Zheng et al., 1999; Zheng et al., 2002).

\section{Breast Cancer Genetics and Susceptibility Genes}

The etiology of breast cancer is driven by multiple components that include environmental factors, physiological host factors, and inherited genetic components. Genes influencing complex diseases are inherited according to Mendelian principles. However, while Mendelian disorders are monogenic, complex diseases are controlled by multiple alleles (Badano and Katsanis, 2002). Genes associated with disease provide a genetic predisposition to development of the disease; however, the outcome is determined by gene-gene interactions and gene-environment interactions (Hunter, 2005; Marian, 2012). At present, the interplay between hereditary and environmental factors is not fully understood (Hunter, 2005). Although it remains difficult to quantify the magnitude of the effect of each of the components on breast cancer etiology, as outlined above, progress is being made to begin to estimate the contribution of the genetic components. 
Women with a family history of breast cancer have an increased breast cancer risk (American Cancer Society, 2011; Bevier et al., 2011; Collaborative Group on Hormonal Factors in Breast Cancer, 2001). RR increases with increasing numbers of first degree relatives diagnosed with breast cancer with $\mathrm{RR}=1.80(99 \% \mathrm{Cl}=1.69-1.91), 2.93(2.36$ 3.64) and $3.90(2.03-7.49)$ for one, two, or three affected first degree relatives, respectively (Collaborative Group on Hormonal Factors in Breast Cancer, 2001). Having an affected second-degree relative increases risk to a lesser degree, with $\mathrm{RR}=1.27(95 \%$ $\mathrm{CI}=1.09-1.47)$ and $1.26(1.05-1.50)$ for either a maternal or paternal grandmother affected, respectively. Further, there is a $R R=1.60(1.24-2.07)$ for two affected second degree female relatives (Bevier et al., 2011). Recent epidemiological studies have revealed that having a brother diagnosed with breast cancer increases a woman's RR to $2.48(95 \% \mathrm{CI}=1.44-4.27)$, which is more than having an affected sister $(\mathrm{RR}=1.87,1.80-$ 1.95). This may suggest that male breast cancer has a higher genetic basis than female breast cancer (Bevier et al., 2011). Clearly, the inherited component affecting breast cancer risk is significant. Moreover, estimates based on breast cancer studies in twins suggest that the inherited genetic component in the etiology of breast cancer accounts for at least $30 \%$ of risk (Lichtenstein et al., 2000). However, Peto (2000) contested that this is an underestimate and that the actual contribution is much higher .

The inherited genetic component of breast cancer in a population consists of both highly penetrant genes at a low frequency and those genes that occur at a high frequency, but have a low penetrance. High risk alleles have been identified in three genes; $B R C A 1$, BRCA2 and TP53 (Lalloo and Evans, 2012). Mutations in these genes are highly penetrant, conferring a $40-85 \%$ increase in lifetime risk for developing breast cancer. 
Additionally, moderately penetrant alleles have been identified in genes such as $P A L B 2$, BRIP1, ATM, and CHEK2 (Meijers-Heijboer et al., 2002; Rahman et al., 2007; Seal et al., 2006; Vahteristo et al., 2002). Variants in these moderately-penetrant alleles confer a 2040\% increase in breast cancer susceptibility (Lalloo and Evans, 2012).

Many mutations and variants in highly- and moderately-penetrant genes are characteristic of specific ethnic backgrounds; therefore, frequencies of these alleles vary across populations due to founder effects and population sizes. For example, mutations in CHEK2 exist in $\sim 1 \%$ of Dutch, Finnish and Ashkanazi Jewish populations, while PALB2 mutations have been identified in Finnish and French-Canadian populations (Erkko et al., 2007; Foulkes et al., 2007; Nevanlinna and Bartek, 2006). In addition, BRCAl/2 mutations have been identified in Ashkanazi Jewish women at frequencies 1050 times higher than those in the general population (Neuhausen et al., 1996; Oddoux et al., 1996; Roa et al., 1996; Struewing et al., 1995). Although variation in these highlyand moderately-penetrant alleles contribute significantly to breast cancer susceptibility, the total population frequency is low with combined frequencies of approximately $0.4 \%$ for $B R C A 1 / B R C A 2 / T P 53$ and $<0.6 \%$ for $P A L B 2 / B R I 1 P / A T M / C H E K 2$ (Lalloo and Evans, 2012). Furthermore, less than half of familial breast cancer patients exhibit predisposing mutations in these genes (Ford et al., 1995; Ford et al., 1998; Smith et al., 2006).

Population-based estimates indicate mutations in high-penetrance genes account for $25 \%$ or less of the heritable component of breast cancer susceptibility (Easton, 1999). This suggests that the majority of heritable risk of developing breast cancer is attributable to additive, dominant, and interactive effects of low-penetrance genes. However, the number and properties of these genes are not known. Much of the work performed to 
identify low-penetrance breast cancer genes has focused primarily on case-control studies targeting potential candidate genes involved in cancer related pathways (de Jong et al., 2002; Nathanson and Weber, 2001). Unfortunately, results of these studies have yet to be validated in larger population-based studies (Wooster and Weber, 2003).

Although useful, risk models that include family history, such as the Gail model, are limited in power. It has been calculated, accounting for no genetic factors, that $62 \%$ of breast cancer cases can be predicted to occur in $50 \%$ of the population at high risk and $15 \%$ can be predicted in the $10 \%$ of the population at highest risk. However, if all of the low-penetrance alleles were known, the ability to predict breast cancer in $50 \%$ of the population at high risk would increase from $62 \%$ to $\sim 90 \%$. This would present the opportunity to detect breast cancer earlier (Pharoah et al., 2002). Therefore, identifying and characterizing common, low-penetrance breast cancer genes will strengthen breast cancer screening and prevention programs.

\section{Common Human Genetic Variation and Breast Cancer}

Genetic variation between individuals occurs at a myriad of sites across the human genome. These variants fall into two broad classes based on their nucleotide composition: single nucleotide polymorphisms (SNPs) and structural variants (Frazer et al., 2009). As the name implies, SNPs are variants at a single base position. To date, more than 12 million SNPs have been catalogued (2005). Structural variants are insertion-deletions (indels), inversions, block substitutions, and copy number variants (Frazer et al., 2009). The ability to effectively detect structural variants has been lacking; 
therefore, much is still not known about the frequency of these variants and their association with human disease. However, current estimates point toward structural variants accounting for $20 \%$ of all genetic variation (Frazer et al., 2009).

Human genetic variants are defined by their minor allele frequency (MAF), which is, simply, the lowest allele frequency that a particular variant is observed in a population. Common variants are defined as having a MAF of $1 \%$ or higher. SNPs are observed more often than structural variants among individuals and it is estimated that approximately 7 million SNPs have a MAF of $5 \%$ or higher with the rest being between $1 \%$ and 5\% (Barrett and Cardon, 2006; Frazer et al., 2009). These SNPs can be used to mark common variation that is hypothesized to underlie genetic susceptibility to developing breast cancer (Fletcher and Houlston, 2010; Frazer et al., 2009; Lee et al., 2011).

Identifying common genetic variants that contribute to disease requires the ability to screen and analyze thousands of variants in a large cohort of individuals in diseased and non-diseased populations. With the advent of high throughput genotyping technologies, population-based genome-wide association studies (GWAS), which assess thousands of SNPs in thousands of individuals, is possible. In another technological advancement, next-generation sequencing techniques that allow for sequencing of large regions of genomic DNA in large sample populations can be incorporated into GWASs. By comparing the genotypes of diseased to non-diseased members of a population, novel genetic determinants may be identified that associate with disease risk. GWASs are now employed to identify novel common genetic variants that associate with an increased susceptibility or resistance to developing breast cancer. Since 2008, 23 breast cancer risk 
GWAS reports have been published using libraries of common polymorphisms to assay genetic variation in human populations (Hindorff et al., 2009). Several common SNPs have been identified in GWAS that associate with increased incidence of breast cancer and these SNPs are located in regions containing novel potential breast cancer susceptibility genes (Easton et al., 2007; Turnbull et al., 2010). Particularly, a GWAS by Easton et al. identified five independent loci that showed a strong correlation with increased breast cancer incidence $\left(\mathrm{P}<10^{-7}\right)$ (Easton et al., 2007). Of these five loci, four contain plausible potential causative genes, i.e. FGFR2, MAP3K1, TNRC9, and LSP1. Although these genes are plausible candidates, further study is necessary to confirm genes contributing to differences in susceptibility.

Further, additional SNPs are often in linkage disequilibrium (LD) with variants that show a positive association to risk. Put differently, particular SNP alleles will frequently be found together in LD and one of these SNPs can be genotyped to "tag" the other SNPs in LD. As a consequence, SNPs used in genome-wide screens may only mark a region carrying an allele associated with a disease phenotype while the causative SNP may be unknown. In other words, the causal variant may not be the one tested in the GWAS. Moreover, tagging SNPs tested in a GWAS may also be in LD with common structural variants, the majority of which have not yet been identified. This underscores one of the limitations of GWAS: there is no simple way of moving beyond statistical association to understanding the functional relationship between a genetic locus and a complex disease phenotype (Frazer et al., 2009).

Another limitation of GWAS is the prospective lack of sufficient statistical power. Many GWAS have been conducted using sample sizes of 2,000 to 5,000 individuals and 
have the statistical power to identify common variants an odds ratio (OR) of 1.5 or higher (Altshuler et al., 2008). To detect variants with an OR of 1.1, studies would require 60,000 individuals to be tested. Although, some of the common genetic variants already identified have per allele OR of $<1.30$ (Ahmed et al., 2009; Antoniou et al., 2010; Cox et al., 2007; Easton et al., 2007; Garcia-Closas et al., 2008b; Milne et al., 2009; Stacey et al., 2008; Thomas et al., 2009; Turnbull et al., 2010) it is likely that many low-penetrance alleles may have been missed due to the small affect on susceptibility. Additionally, novel or rare variants may also be missed by GWAS. Thus, although GWAS have been useful in identifying some common genetic variants associated with breast cancer susceptibility, new approaches are needed to effectively study common, low-penetrant alleles and their effect on disease susceptibility.

\section{Gene-Environment Interactions}

Further complicating studies of breast cancer susceptibility is the combined action of risk-predisposing genes and environmental factors. Aside from studies on genetic variants in detoxifying metabolic enzymes, relatively little is known about the majority of genetic risk alleles and their potential interactions with the environment (Masson et al., 2005). Recent studies have implicated potential interactions between $F G F R 2$ variants and hormone replacement therapy; however, these data are inconsistent (Prentice et al., 2009; Travis et al., 2010). Additionally, a study was published on potential interactions between ten environmental risk factors (age at menarche, parity, age at first birth, breastfeeding, menopausal status, age at natural menopause, hormone replacement therapy, body-mass index, height, alcohol consumption) and twelve breast cancer 
susceptibility-associated SNPs (Travis et al., 2010). No strong interaction was identified between any of the 120 possibilities. However, the majority of women in this study were post-menopausal, and therefore, these data may miss gene-environment interactions occurring in younger women. Also, moderate modulations may be occurring that are not being detected. Detecting a gene-environment interaction requires a four-fold larger sample size than does a main effect; thus, for a $R R=\sim 1.5$ thousands of cases and controls or tens of thousands of GWAS cases would need to be assessed (Thomas, 2010). This study examined 7610 women with breast cancer and 10,196 controls; although a large sample size, the authors acknowledge it may be insufficient to identify moderate effects (Travis et al., 2010). Furthermore, these studies are somewhat limited in that they exclude certain environmental components; i.e. pollutants such as BPA, PHCs and PCBs. Exposure to these environmental pollutants is becoming more common and it is relevant to assess the effect of exogenous chemical exposure in the environment on breast cancerpredisposing alleles.

\section{Animal Models of Breast Cancer Susceptibility}

\section{Mouse Models for Breast Cancer Research}

Human studies provide the most direct way of studying breast cancer susceptibility. However, different approaches are needed to identify the effects of lowpenetrant alleles. Human cell lines are routinely used to study breast cancer development, progression and metastasis (Burdall et al., 2003). They have a number of advantages; they are easy to handle and represent a potential unlimited self-replicating source that can be grown in almost infinite quantities. In addition, they exhibit a 
relatively high degree of homogeneity and are easily replaced from frozen stocks if lost through contamination. There are disadvantages though, as cell lines are prone to genotypic and phenotypic drift due to continual culture. This occurs as subpopulations may arise over time by the selection of specific, more rapidly growing clones within a population leading to phenotypic changes (Bahia et al., 2002; Osborne et al., 1987). In addition to cell growth rate, changes have been observed in hormone receptor content, karyotype and clonogenicity, despite the cells appearing morphologically identical. However, the most important weakness for susceptibility studies is that cell lines are usually derived from tumors and have adapted to growth in culture. Although cell culture tries to create a close-to-physiology milieu by adding appropriate amounts of salt, glucose, amino acids, vitamins, and serum, the lack of tissue architecture and heterogeneous population of cell types often abolishes cell-cell interaction, secretion, and other functions based on tissue context (Pan et al., 2009). As stated, cells in culture are prone to genotypic and phenotypic drifting. Thereby cell lines can lose tissue-specific functions and acquire a molecular phenotype quite different from cells in vivo. Thus, cell lines are limited in scope and cannot fully replicate the disease phenotype. Although they can be used to study specific molecular targets in transformed cells they are not an effective model to study breast cancer susceptibility.

Use of animal models has been an effective approach for studying human diseases. Various model organisms have been successfully used to study aspects of different human diseases in vivo. The most widely used animal models of breast cancer are the mouse and the rat. Mouse models have the benefit that more genetic manipulation techniques exist for them compared to the rat. Many transgenic models have been 
developed taking advantage of the long terminal repeat (LTR) of the mouse mammary tumor virus (MMTV) (Hutchinson and Muller, 2000). The MMTV-LTR is active in the mammary gland and was shown to be able to promote expression of genes in a mammary gland-specific manner (Cardiff and Kenney, 2007). The MMTV-Polyoma virus middle T antigen MMTV/PyV mT mouse model is one of the most widely used experimental animal models used to study mammary tumor development and metastasis. In this model, the mT antigen derived from PyV was placed under the transcriptional control of the MMTV-LTR (Guy et al., 1992a). The mT antigen was identified to induce multifocal tumors in mammary glands through activation of various signaling molecules such as Src family kinases and phosphatidylinositol 3' kinase (PI3K) in mice (Guy et al., 1992a). This mouse model develops polyclonal tumors aggressively within 7-8 weeks with a high degree of lung metastasis (Marcotte and Muller, 2008). The MMTV/PyV mT mouse model is often used in combination with gene knockouts to examine the influence they may have during mammary carcinogenesis or metastasis.

Another widely used transgenic mouse model is the $H E R 2 /$ neu or ErbB2 mouse. $H E R 2$ codes for a tyrosine kinase growth factor receptor and is the human ortholog of the rat neu oncogene that was identified to increase neuroblastoma development (Coussens et al., 1985; Schechter et al., 1985). Since it was identified in humans, HER2 has been identified to be overexpressed in $20-30 \%$ of human breast cancers and has become a prime therapy target for treating breast cancer (Wang et al., 2000). The mouse ortholog of $H E R 2 / n e u$ is $E r b B 2$. ErbB2 was identified to be an oncogene in studies using transgenic mice that overexpressed activated neu under the control of an MMTV promoter. While mammary epithelial expression of activated neu is sufficient to induce 
mammary tumorigenesis, few activating mutations in HER2 have been identified in humans, suggesting it exerts oncogenic effects through overexpression of the wild-type Her2 receptor (Lemoine et al., 1990). The ErbB2 transgenic mouse model of breast cancer was developed with a transgene carrying the wild-type $\operatorname{ErbB2}$ proto-oncogene under the control of the MMTV promoter (Guy et al., 1992b). Overexpression of wildtype $E r b B 2$ results in multifocal tumors, but they occur with a longer latency compared to the MMTV-PyV mouse model of breast cancer. Further, MMTV-ErbB2-induced mammary tumors are less metastatic than MMTV-PvV-induced tumors (Guy et al., 1992b). Regardless, the ErbB2 transgenic mouse model has been used extensively to examine the role of the ErbB2 proto-oncogene in mammary tumor development. In addition, the MMTV system has been extensively used to study other proto-oncogenes, e.g., c-myc and cyclin D1 (Stewart et al., 1984; Wang et al., 1994). Overall, the use of the MMTV transgenic mouse system has led to a greater understanding of the genetic machinery of mammary carcinogenesis.

In addition to transgenic mouse models, gene knockout mice are regularly used and these have been combined with 'knock-in' and conditional tissue-specific gene targeting technologies allowing for a wide range of approaches to study human disease (Hutchinson and Muller, 2000; Maddison and Clarke, 2005). These studies involve deleting or inserting specific genes of interest into a targeted region using targeted recombination. Moreover, the development of the Cre-Lox system has allowed for conditional and/or temporal deletion of target genes (Maddison and Clarke, 2005). With this method, specific regions of DNA, e.g. whole genes, exons, promoters, are flanked by specific pieces of DNA termed loxP sites oriented in the same direction. When crossed 
with mice carrying the Cre recombinase gene, Cre expression carries out loxP-specific recombination in a topoisomerase-like manner; thereby cleaving out the intervening sequence between the two loxP sites (Maddison and Clarke, 2005). When Cre is under the control of a tissue- or development-specific promoter, the knockout is directed to specific sites and/or at specific points in development. Also, inducible systems have been developed to control expression of genes when administered a specific inducer. Gossen and Bujard developed the tetracycline (tet)-dependent transcriptional activation system allowing spatial and temporal control of effector gene expression through the use of a tissue-specific transactivator (Gossen and Bujard, 1992). When an effector gene is under the control of the tet system, expression is activated only when the mouse is administered tetracycline in the diet. Therefore, gene expression can be easily turned on or off. Moreover, Cre can be placed within a tet-dependent system making it inducible (Gossen and Bujard, 1992; Maddison and Clarke, 2005). This way, the knockout only occurs once the mouse has been administered tetracycline. However, the Cre-Lox system has been found to have "off target" effects where Cre acts on lox-like sites causing inadvertent deletion (Maddison and Clarke, 2005). Regardless, the Cre-Lox and the tetinducible systems have been useful in dissecting pathways of genes involved in a host of diseases.

A genetic "tool box" has been developed for the mouse making it a useful model to study the genetic and molecular aspects of human disease. The mouse has been instrumental in identifying mechanisms of specific genes involved in mammary carcinogenesis and this has increased our knowledge of these genes in human breast cancer. There are additional models and technologies not mentioned that are being 
employed to study many human diseases including breast cancer. Furthermore, new technologies for using the mouse continue to be developed.

\section{Rat Models of Breast Cancer Susceptibility}

Although mice are widely used to study mammary carcinogenesis, there are some key differences between mice and humans in the pathology of mammary and breast tumors that each species develops. First, $\sim 50 \%$ of breast carcinomas arising in humans are $\mathrm{ER} \alpha$ positive and thus are hormonally responsive while mouse mammary tumors are almost exclusively ER $\alpha$ negative and hormone independent (Marcotte and Muller, 2008). Also, human mammary gland tissue is primarily made up of connective tissue, while mouse mammary gland stroma consists mainly of adipocytes (Marcotte and Muller, 2008). Thus, mouse model studies on mammary carcinogenesis are lacking in their ability to translate to human breast cancer.

The laboratory rat is preferable for the study of breast cancer susceptibility because the pathology of rat mammary cancer is more similar to human breast cancer. Although mouse models clearly have merit in understanding human disease, rats are more similar to humans than mice in their normal physiology and pathogenesis. Both rats and humans develop tumors that arise in epithelial cells lining the mammary gland duct have similar histopathology, and both develop the same proportion of hormonedependent and endocrine therapy, e.g., tamoxifen, -responsive carcinomas (Gould, 1995). This is contrary to the mouse where most mammary tumors are hormonally refractive and many tumors are of endothelial cell origin (Gould, 1995). Additionally, while a majority of mammary tumors that form in mice are induced by MMTV, rats and humans do not form mammary tumors with a known viral etiology (Gould, 1995). Finally, and most 
importantly for mammary gland susceptibility studies, rats and humans both have a natural genetic variation in mammary and breast carcinoma susceptibility (Dunning and Curtis, 1952; Dunning et al., 1947; Gould, 1995; Isaacs, 1986; Isaacs, 1988). These similarities make the rat an ideal model to study natural variation in development of mammary carcinogenesis.

Various rat models have been developed to study breast cancer susceptibility. Age and gender are the most widely accepted risk factors affecting breast cancer development and this has been attributed to ovarian hormone exposure. Dunning et al. discovered that the ACI rat strain was susceptible to estrogen-induced mammary cancers (Dunning and Curtis, 1952; Dunning et al., 1947; Dunning et al., 1953; Shull et al., 1997). Conversely, Copenhagen (COP) rats are almost completely resistant to developing estrogen-induced mammary tumors. In this regard, $\mathrm{ACl}$ and $\mathrm{COP}$ rats are commonly used together to study estrogen action in the rat. Specifically, ACI female X COP male and $\mathrm{COP}$ female $\mathrm{X} \mathrm{ACI}$ female intercrosses were performed to generate $\mathrm{F} 1 \mathrm{a}$ and $\mathrm{F} 1 \mathrm{~b}$ progeny, respectively. F1 progeny were administered E2 to induce carcinogenesis and tested for mammary carcinoma susceptibility. Data indicated no significant difference in tumor incidence between Fla, Flb or homozygous ACI rats. However, latency to appearance of the first E2-induced mammary tumor was significantly prolonged in both F1 populations compared to ACI homozygous rats (Shull et al., 2001). Further, siblings from $\mathrm{F} 1 \mathrm{a}$ and $\mathrm{F} 1 \mathrm{~b}$ progeny were mated to each other to generate $\mathrm{F} 2 \mathrm{a}$ and $\mathrm{F} 2 \mathrm{~b}$ progeny, respectively, and F1a and F1b males were mated back to ACI females to generate backcrosses a ( $\mathrm{BCa})$ and $\mathrm{BCb}$ progeny, respectively. Using these lines a locus was mapped on chromosome 5 that conferred susceptibility to estrogen-induced mammary 
carcinoma development. This method was further utilized to identify multiple quantitative trait loci (QTLs) in the ACI rat responsible for estrogen-induced mammary carcinoma susceptibility (Gould et al., 2004; Kurz et al., 2008; Shull et al., 2001).

Other rat lines have varying propensities for developing exogenous non-estrogen carcinogen-induced mammary carcinomas. The Wistar Furth (WF) rat strain was identified to be susceptible to developing spontaneous, radiation-, oncogene-, and carcinogen-induced mammary carcinomas (Gould, 1986; Moore et al., 1983). Conversely, the COP rat strain is susceptible to ionizing radiation and oncogene-induced mammary carcinogenesis, but is almost completely resistant to developing chemical carcinogen-induced mammary tumors (Isaacs, 1988). Studies using ionizing radiation on (WF X COP) F1 rats detected random allelic imbalances throughout the genome leading to development of mammary tumors with no preferential loss for either the WF or COP parental alleles (Haag et al., 1996). This indicates that neither the WF nor COP allele confer resistance to radiation-induced mammary carcinogenesis. Additionally, both WF and COP females treated with a v-H-ras oncogene-containing retrovirus directly infused into the mammary gland develop similar tumor incidences (Wang et al., 1991). As previously mentioned, COP rats are resistant to estrogen-induced tumors; however, hormonal promotion enhanced the penetrance of the ras oncogene on mammary tumor formation in both COP and WF female rats in a similar manner (Wang et al., 1991). Although the COP and WF rats showed similar tumor incidence from ras oncogene induction, COP tumors were more differentiated and less invasive than WF tumors (Wang et al., 1991). This suggests that although oncogene activation in situ was able to 
ultimately overcome the resistance phenotype, COP rats still exhibit a less malignant phenotype compared to WF rats.

\section{Mammary Carcinoma Susceptibility Ouantitative Trait Loci}

\section{Mammary Carcinoma Susceptibility Alleles}

The rat is not as easily genetically manipulated as the mouse. Until recently, chemical or random-insertional mutagenesis screens had been employed in the rat to identify causative genes for specific phenotypes (Aitman et al., 2008). Now, transgenic rat models are available and it is possible to generate knockout lines (Aitman et al., 2008; Geurts et al., 2009). However, these approaches are biased towards particular genes of interest or involve disrupting the genetic architecture by random insertion of foreign DNA into the chromosome. Although these methods may be used for validating or studying the effects of specific genes, they are not suited for forward genetics studies focused on identifying genotypes responsible for disease susceptibility phenotypes. Instead, congenic rat lines have been developed that allow one to study mammary carcinoma susceptibility in a "natural" context. Congenic lines take advantage of varying susceptibility phenotypes between different rat strains. Congenic rat lines for mammary carcinoma susceptibility studies are developed by introgressing alleles from a resistant strain onto a susceptible strain by first identifying an allele of interest in an $\mathrm{F} 1$ population and then continuously backcrossing to a recipient strain. By genotyping and selecting for the donor allele of interest, the donor allele is retained and introgressed onto the genotype of the recipient background strain (Figure 1) (Markel et al., 1997). Lines with different segments of the donor allele can be tested to map a genetic locus to define the allele of interest. 


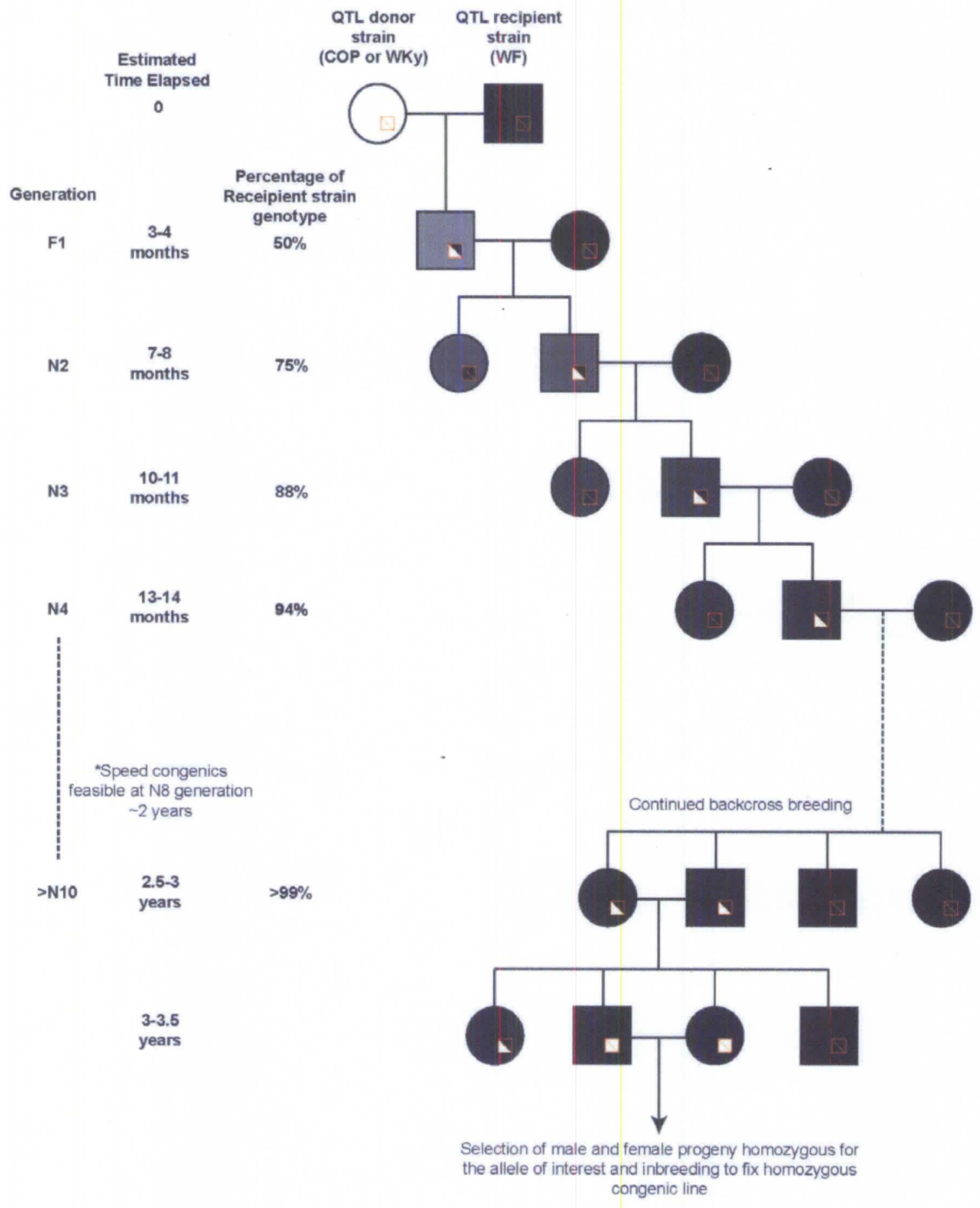


Figure 1: Congenic Breeding Scheme. Homozygous susceptible male rats (WF) are bred to female resistant rats $(\mathrm{COP} / \mathrm{WKy} / \mathrm{BN})$ to create an $\mathrm{F} 1$ heterozygous population. F1 progeny are genotyped for the allele of interest. F1 males containing the allele of interest are backcrossed to WF females to produce the N2 population. At each subsequent generation the litters are genotyped and males carrying the allele of interest are backcrossed again to WF females. At the N8 generation, speed congenics are potentially viable to use for mapping as the background genotype is $>99 \%$. However, backcrossing to $\mathrm{N} 10$ is common. At N10 (or N8) heterozygous male and female littermates are inbred and the offspring are genotyped to identify pups homozygous for the target allele. These homozygotes are inbred continuously to fix the line for the allele of interest. $\bigcirc$ and represent resistant female, susceptible male, and susceptible female rats, respectively. The gray shade of a circle or square depicts the genotype percentage of the background genome in heterozygous offspring; i.e. as the percentage of the background genome becomes more WF, the shade becomes darker gray. $\mathbf{\Delta}, \nabla$, and I inside a circle or square represent the target allele when it is homozygous WF, homozygous COP, and heterozygous, respectively. 
Work by our group employs congenic strains developed with resistant alleles COP and Wistar Kyoto (WKy) donor strains on a WF background denoted as WF.COP and WF.WKy congenic lines, respectively. In contrast to WF rats, COP and WKy rats have been shown to be almost completely resistant to developing mammary carcinomas induced by the chemical carcinogens DMBA and $N$-Nitroso- $N$-methylurea (NMU) (Haag et al., 1992; Isaacs, 1986).

WF.WKy and WF.COP have been used to identify novel independent loci affecting mammary carcinoma susceptibility (Cotroneo et al., 2006; Haag et al., 2003; Lan et al., 2001; Samuelson et al., 2003). These QTLs have been denoted as mammary carcinoma susceptibility $(M c s)$ loci. The first $M c s$ locus, $M c s 1$, was found in a DMBAinduced carcinogenesis study on female progeny from a (WF X COP)F1 X WF backcross (Hsu et al., 1994). Since then, WF.COP and WF.WKy congenic lines have been used to identify other Mcs loci: Mcs2, Mcs3, and Mcs4, all found in WF.COP congenics, and $M c s 5, M c s 6, M c s 7$ and $M c s 8$, found in WF.WKy congenics (Lan et al., 2001; Shepel et al., 1998). Each strain, COP or WKy, has three QTLs that increase and one that decreases susceptibility to developing DMBA-induced mammary tumors. Only one QTL in each strain overlaps with a QTL at the same genetic locus in the other strain. These are COP Mcs2 and WKy Mcs6. This illustrates the genetic diversity of mammary carcinoma genetic susceptibility as both of these strains are highly resistant to developing DMBA-induced mammary carcinomas.

\section{Mammary Carcinoma Susceptibility Locus 1 b (Mcs1b)}


As stated above, (WF X COP)F1 X WF backcrosses were used to identify the Mcs 1 locus. Rats were divided into groups based on susceptibility: resistant (R), undetermined $(\mathrm{U})$, and susceptible $(\mathrm{S})$. Rats from R and S groups were genotyped using microsatellite markers and the locus conferring resistance was determined to be on the proximal end of chromosome 2 (Hsu et al., 1994). Resistant congenic F1 rats were backcrossed to WF rats and progeny were genotyped to identify shorter regions of the interval to fine map the $M c s I$ locus. Results of phenotyping showed that the Mcs $I$ locus contained 3 independent loci that each reduced DMBA-induced tumor incidence by $\sim 60 \%$ compared to homozygous WF controls (Haag et al., 2003). These were termed $M c s l a, M c s l b$, and Mcslc (Figure 2). Interestingly, one of these loci, Mcs $1 b$, contains a region orthologous to a human locus identified in a GWAS of breast cancer susceptibility (Easton et al., 2007).

Easton et al. (2007) reported the identification and validation of five novel breast cancer susceptibility loci in a 3-stage breast cancer GWAS (Easton et al., 2007). One of these SNPs, $r s 889312$, has MAF of 0.38 with an allelic OR of $1.13(95 \% \mathrm{CI}=1.10-1.16)$ and associates with an increase in breast cancer incidence $\left(\mathrm{P}=7 \times 10^{-20}\right)$. Additionally, SNP rs 889312 localizes to a region on human chromosome 5 and is in LD with 6 other SNPs contained within a $280 \mathrm{~kb}$ haplotype block (Figure 3)(Barrett et al., 2005). Importantly, this human haplotype block is orthologous to a region within the rat $M c s l b$ locus. There are three transcripts annotated to the human locus delineated by the $280 \mathrm{~kb}$ haplotype block; $M A P 3 K 1, M I E R 3$, and C5ORF35 (Figure 3). These transcripts are also annotated to the rat $M c s I b$ locus. In addition there are 7 more transcripts annotated that lie nearby, but outside the $280 \mathrm{~kb}$ haplotype block on the human locus, that are also 


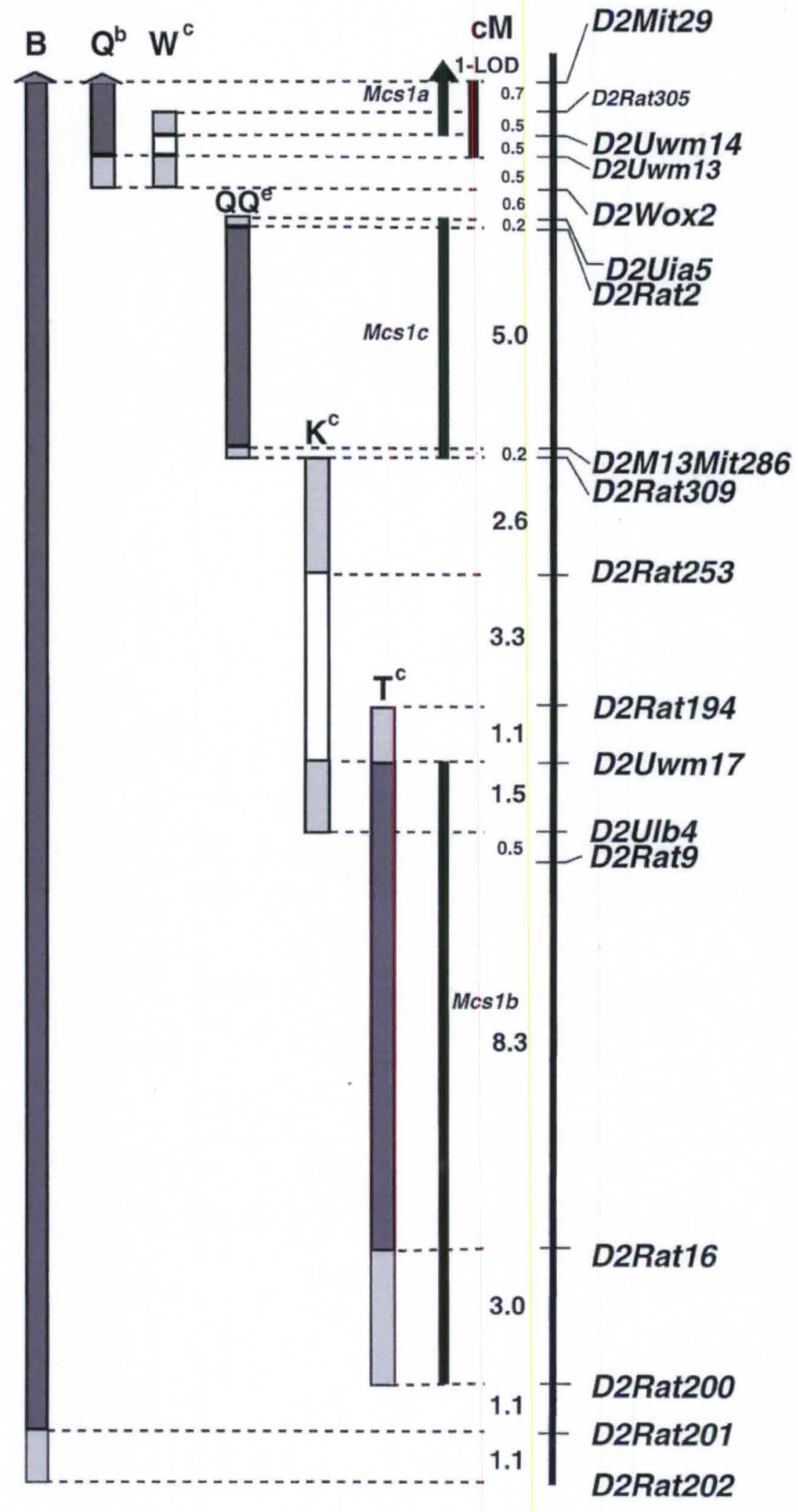

Haag et al. Cancer Research, 2003 
Figure 2: Chromosome 2 genetic map of Mcs 1-congenic and -recombinant rat lines. Rat lines are designated with capital letters. $\square$, indicates the presence of two COP alleles for congenic lines resulting in reduced mammary tumor development (one to four carcinomas/rat); $\square$ indicates the presence of two COP alleles for congenic lines incapable of conferring resistance to tumor development (six to eight carcinomas/rat). $\square$, indicates areas of unknown genotype because of recombination. Three independent regions of chromosome 2 were capable of conferring resistance to mammary cancer development in COP-homozygous congenic rats. These genomic regions are shown as Mcsla, Mcs 1b, and Mcslc. The chromosome markers used to identify the congenic rats are listed to the far right of the figure with genetic distances in centiMorgans (cM) to the left of the marker names. The Mcsl QTL 1-LOD interval is also shown between D2Mit29 and D2Uwm13. 


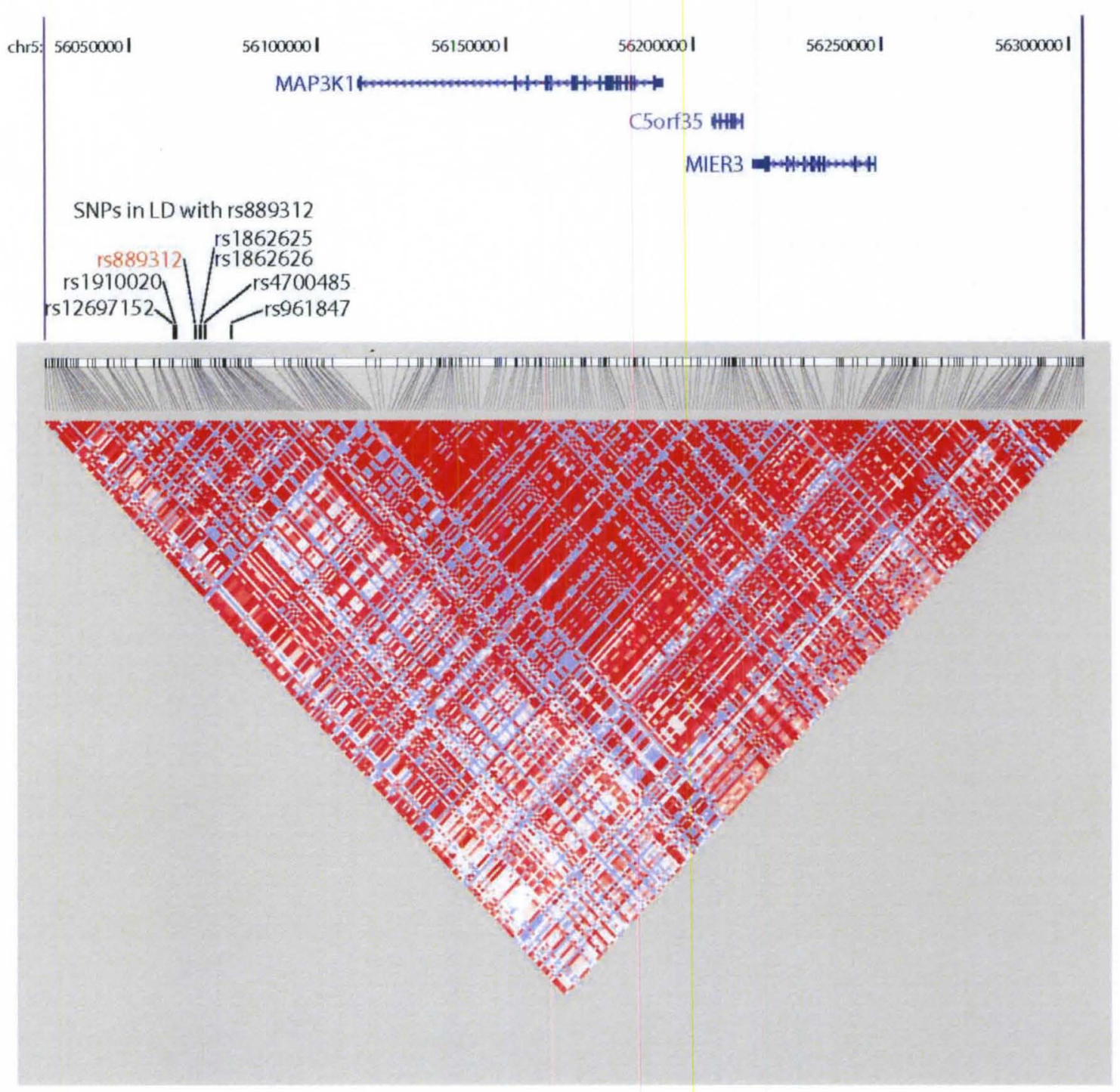

Figure 3: $280 \mathrm{~Kb}$ haplotype block containing breast cancer-associated SNP rs889312. Transcripts are shown in blue with exons designated as vertical bars. Above, the location of the tagging SNP $r s 889312$ is shown in red, other SNPs in LD with $r s 889312$ are shown in black. Below, pairwise $\mathrm{r}^{2}$ values for LD are shown; light blue represents lower disequilibrium, white is intermediate, and red represents strong disequilibrium. An $\mathrm{r}^{2}$ threshold of 0.80 was used to determine SNPs in LD in this block. 
contained in the rat Mcs1b locus; GPBPI, IL3IRA, IL6ST, DDX4, ANKRD55, ACTBL2, and SLC38A9. MAP3K1 has been described as the most likely candidate gene due to its role as a protein kinase and involvement in promoting cell growth and proliferation; still, any of these genes could be involved in affecting breast cancer susceptibility in humans. Furthermore, any of the SNPs or combination of variants in LD with $r s 889312$ could be causative to affect breast cancer susceptibility. Therefore, each SNP must be studied to identify the causative variant. The original study identifying this human locus consisted of an initial two-stage GWAS on 4,398 breast cancer cases and 4,316 controls, followed by a third stage testing 21,860 cases and 22,578 controls from 22 studies (Easton et al., 2007). Testing individual SNPs in LD with $r s 889312$ in this population would offer a better view of the genetic composition associated with this phenotype; however, this provides no insight into their function. Studying the molecular mechanisms by which these SNPs are operating in a human population is not realistic. Therefore, the WF.COP $M c s 1 b$ congenic rat line is a good model to study this locus in the context of breast cancer susceptibility.

There are over 50 transcripts annotated within the rat $M c s 1 b$ locus. It is anticipated that the causative gene is conserved between humans and rats which reduces the number of viable gene candidates to be tested. Nonetheless, all of the transcripts annotated to the current rat $M c s l b$ locus must be treated as potentially causative; thus, all genes must be tested. It is useful, then, to attempt to reduce the number of genes to be tested by narrowing the current rat $M c s I b$ locus by means of positional mapping. The rat genome has been sequenced and many microsatellite and SNP markers are available to effectively map $M c s 1 b$ to a narrower interval. Testing genes in the rat may identify the 
causative gene involved at the human locus. Related to this, the $M c s 1 b$ rat model can also be used to study the cellular and physiological phenotypes affecting mammary carcinogenesis associated with this locus. On the whole, a $M c s 1 b$ rat genetic model could help determine mechanisms controlling human breast cancer that are associated with the locus demarcated by SNP rs889312 and expand our current understanding of breast cancer etiology.

\section{Dissertation Overview}

\section{Overall Goal}

The work presented in this dissertation is focused on using congenic WF.COP $M c s I b$ rat lines to study mammary carcinoma susceptibility. I made use of the varying propensity for developing mammary tumors between WF.COP Mcs $1 b$ congenic female rats and homozygous WF female rats. The overall goal was to narrow the genetic interval and to identify potential gene candidates. Mapping the $M c s l b$ locus to a shorter interval reduces the number of potential gene candidates and focuses on identifying the causative gene(s). Identification of the causative gene(s) could lead to development of novel genetic tests for better diagnosis as well as novel targets for prevention and treatment of breast cancer. In addition, I began to identify the cellular mechanism by which rat Mcs $1 b$ affects mammary carcinogenesis and tumor development. By elucidating the means by which this susceptibility locus is working, we will increase the current understanding of breast cancer etiology.

\section{Hypothesis and Research Aims}


I hypothesized that the rat Mcs $1 b$ locus contains genetic elements controlling molecular and cellular phenotypes that translate into mammary carcinoma susceptibility phenotypes. By completing aim one; I successfully delimited the rat $M c s I b$ locus to a region of $1.1 \mathrm{Mb}$ containing three genes. In aim two, I identified that the mammary carcinogenesis susceptibility allele is acting in a mammary gland cell-autonomous manner. In aim three, I show that the transcript Mier3 is a candidate modifier gene controlling mammary carcinogenesis based on its differential transcript expression between susceptible and resistant rat strains. These data will help us better understand breast cancer etiology and may lead to better diagnoses and treatments of human breast cancer. 


\section{CHAPTER II}

\section{RAT MCSIB IS WITHIN A 1 MB REGION OF RAT CHROMOSOME 2}

\section{Introduction}

The rat Mcs 1 locus was originally identified on the proximal end of chromosome 2 in (WF $x$ COP)F1 $x$ WF backcrosses using DMBA to induce mammary carcinomas (Hsu et al., 1994). Previously, the Mcs 1 locus was physically confirmed and positionally mapped using congenic lines containing unique segments of the Mcs l predicted QTL interval. Female rats from these lines were tested using the same DMBA carcinogenesis protocol and revealed that the locus contained three independent susceptibility loci that conferred resistance to developing mammary carcinomas. These QTLs were termed Mcsla, Mcs $1 b$ and Mcs $1 c$ (Haag et al., 2003)(Figure 2).

The Mcs $1 b$ locus is of particular interest because it is orthologous to a human locus associated with increased breast cancer incidence that was identified in a GWAS (Easton et al., 2007). The human breast cancer risk-associated allele was tagged with SNP rs 889312 which lies within a $280 \mathrm{~kb}$ LD block on human chromosome 5 . This LD block contains three annotated transcripts; MAP3K1, MIER3, and C5ORF35 (Figure 3). The rat ortholog to this locus is contained in the $M c s I b$ congenic line $\mathrm{T}$ interval, which was mapped to a region delimited from marker D2Uwm17 to D2Mco42 (Chr2: 32051319

- 45248161). This locus contains over 50 annotated transcripts, including Map3kl, Mier3, and C5orf35. Mapping rat Mcs $1 b$ to a narrower region will reduce the number of 
candidate genetic elements to be functionally tested and minimize the work required for comparative genetic studies.

Many QTLs are multigenic or affected by more than one genetic element. For that reason, two or more elements may potentially act in concert to affect susceptibility. Narrowing the interval may provide insight into the function of this locus. Accordingly, I attempted to map the $M c s I b$ locus to a tighter interval using a congenic mapping approach previously used to segregate and identify three independent loci contained within the original Mcsl locus (Haag et al., 2003). This approach will reduce the genetic elements to be functionally tested as well as potentially identify complex genetic interactions or additional risk alleles within the line T $M c s l b$ interval.

The advantages of the rat over a mouse model have been noted. However, there are other rat-centric methods that could be employed, e.g., transgenic rat models, rather than the congenic approach used in these studies. Many genes are annotated to the rat $M c s 1 b$ locus, as it is currently defined, and these could be tested in a transgenic rat model by introducing the genes as transgenes under the control of an artificial promoter. However, this approach would be extremely labor-intensive requiring the development of over 50 transgenic lines. Further, gene regulatory elements cannot be cloned into traditional transgenic cassettes. This is critical, since we do not know the transcript profile of the hypothesized candidate gene. In this regard, bacterial artificial chromosome (BAC) transgenic rats may be useful. BAC transgenics allow for up to 400 $\mathrm{kb}$ of foreign DNA to be cloned so that all the regulatory elements may be included. For this reason, BAC transgenes are expressed in a more spatially, temporally, and physiologically accurate manner. The current $M c s I b$ locus is delineated to a $\sim 15 \mathrm{Mb}$ 
interval containing 50 transcripts; thus, much like traditional transgenics, the BAC transgene method would also require many lines to be developed to tile across the region. Moreover, if there are multiple components involved, using a BAC transgenic may "hide" these effects. Another shortcoming of this method is that the BAC transgene is incorporated randomly in the genome. Although regulatory elements and surrounding DNA are included, this technique disregards possible long range effects, e.g., distal enhancers and long range cis regulatory elements, thereby altering the context.

Although laborious and time-consuming, the congenic rat model is the preferred approach. It is powerful in that it is unbiased with regard to the cause of action, i.e., the heritable element(s) controlling the susceptibility phenotype may be non-protein coding regulatory elements that control expression or processing, causative elements may be in cis of secondary element, or they may be multigenic and require multiple factors. Overall, congenics allow one to study the action of the Mcs $l b$ locus in its native physiologically-relevant environment. For these reasons, I continued to use the previously-described congenic approach for these studies.

Until now, the rat $M c s 1 b$ region has been genotyped using microsatellite markers. The genome sequence for the rat is available and groups are attempting to develop SNP maps of the rat genome (Nijman et al., 2008; Saar et al., 2008). However, the annotated sequence is based on the Brown Norway $(\mathrm{BN})$ strain, and the COP and WF genomes were not included in SNP and haplotype studies; therefore, there are no well-documented polymorphisms between the COP and WF lines in the Mcslb region. Identifying new polymorphisms between the WF and COP strains will provide better markers to more accurately map the Mcs $1 b$ locus. Moreover, within the $280 \mathrm{~kb}$ haplotype block 
containing the human Mcslb ortholog, SNP rs889312 is in LD with 6 other annotated SNPs: $r s 12697152, r s 1910020, r s 1862625, r s 1862626, r s 4700485$, and $r s 961847$ (Figure 3). Any of the SNPs lying in the $280 \mathrm{~kb}$ LD block could be causative. However, it is not clear which of these SNPs, if any, is the causative variant responsible for conferring breast cancer susceptibility. Rat orthologs to the 7 human SNPs lying within the LD block are not known. Consequently, identifying novel polymorphisms between the WF and COP rat lines could potentially lead to identifying the causative SNP controlling mammary carcinoma susceptibility and comparative genetics could be used to accurately identify the causative SNP in the human genome.

Overall, identifying novel polymorphisms between the WF and COP strains will add to the current knowledge of these strains and can be used for the mapping studies described herein. These SNPs are potentially useful in that they may shed light on the causative element responsible for the difference in mammary carcinoma susceptibility between these two rat lines and identify the causative variant in the human genome. Fine-mapping this region in the rat will reduce the number of potential gene candidates that must be functionally evaluated. As stated, this approach is powerful in that it is unbiased with regards to the cause of action, i.e., the heritable element(s) controlling the susceptibility phenotype may be a regulatory element(s) controlling expression or processing of a transcript. In addition, these elements may lie within or outside of the protein coding region of the gene. Indeed, all of the SNPs in the human breast cancerassociated haplotype block lie outside of the transcribed regions of any of the annotated transcripts. Furthermore, this approach may uncover complex genetic interactions and/or potential additional mammary carcinoma susceptibility loci that lie within the region. 


\section{Design and Methods}

\section{Resequencing}

WF and COP rats were euthanized and splenectomized using a protocol approved by the University of Louisville Institutional Animal Care and Use Committee (IACUC). Spleens were frozen until DNA was isolated. DNA was isolated using a Gentra Tissue DNA Extraction Kit (Gentra, Minneapolis, MN) and PCR amplified using AccuPrime Taq polymerase (Life Technologies, Grand Island, NY) at specific regions defined in an article by Cuppen and colleagues (Nijman et al., 2008) or compiled in the STAR Consortium SNP database (http://www.snp-star.eu/). These PCR products were purified using a QIAquick PCR Purification Kit (QIAGEN, Valencia, CA) and sequenced using the BigDye Terminator v3.1 Cycle Sequencing Kit (Life Technologies). Sequencing reaction products were purified with $5 \mu$ Agencourt AMPure XP beads and $80 \%$ ethanol. Beads were washed once with $80 \%$ ethanol and subsequently eluted in molecular biology grade water. Sequence products were submitted for analysis to the University of Louisville Center for Genetics \& Molecular Medicine DNA Core using an ABI PRISM 3130XL Sequence Detection System (Life Technologies).

Additionally, random regions across the $M c s l b$ locus were selected to be amplified and sequenced to identify polymorphisms. Using the UCSC genome browser, regions exhibiting stretches of di- and tri-nucleotide repeats were selected and primers were designed using Primer3 (Rozen and Skaletsky, 2000) oligonucleotide design software. Primers were obtained from Integrated DNA Technologies (IDT, Coralville, IA). Using these primers, genomic DNA (gDNA) was PCR amplified and run on highresolution agarose gels to identify potential microsatellite polymorphisms between WF 
and COP sequence. Additionally, long spans were selected randomly across the Mcs $1 b$ locus and PCR amplified and sequenced to identify potential SNPs between WF and COP sequence. Sequence reads were analyzed using DNAStar Sequence Analysis software (DNAStar, Madison, WI).

\section{Congenic Strain Breeding}

The congenic breeding method was detailed above (Figure 1). The rat WF.COP $M c s 1 b$ congenic lines $\mathrm{T}$ and $\mathrm{B}$ were used to generate new recombinant lines and potentially isolate the SNP(s) and/or gene(s) involved in the breast cancer susceptibility phenotype. These lines, containing varying pieces of the rat Mcs $1 b$ locus, will be tested using the DMBA mammary carcinoma susceptibility assay. The line $\mathrm{T} M c s 1 b$ locus was delineated to $\sim 13 \mathrm{Mb}$ interval at D2Uwm17:g2UL2-30 (Chr2:32051320-44932309) and confers a reduction in mammary tumor development (Figure 2) (Haag et al., 2003). Line B was delineated a Chr2 region from marker D2Mit29 to marker D2Rat201 Figure 2. In addition to $M c s l b$, the Line B COP interval contains Mcsla and Mcslc; however, the recombinant lines at the distal end may be used to narrow the $M c s 1 b$ locus.

Inbred WF male and female rats were obtained from Harlan Sprague Dawley, Inc. (Indianapolis, IN). All housing and breeding was performed in the Research Resources Center Animal Facility at the University of Louisville under protocols approved by the University of Louisville Animal Care and Use Committee. Line T male and female rats at the N10 generation or beyond were bred with inbred homozygous WF female or male rats, respectively, at 12 weeks of age. Progeny were genotyped for unique recombinant intervals of the $M c s I b$ locus. Novel recombinant rats were backcrossed with inbred 
homozygous WF rats to obtain heterozygous progeny. Heterozygous male and female progeny were inbred to obtain progeny homozygous for the unique recombinant allele.

WF.BN-RNO2 animals were generated in a similar manner by initially breeding BN female rats (Harlan Sprague Dawley, Indianapolis, IN) to a WF male rat to obtain an F1 generation. An F1 generation male was backcrossed to inbred (homozygous) WF female rats and progeny were screened for the $M c s 1 b$ locus using the markers mentioned. WF.BN animals were bred out to the N6 generation. Mammary carcinoma susceptibility phenotype studies were performed as described.

\section{Genotype Analysis}

Progeny from crosses between congenic and homozygous WF rats were genotyped using an approved protocol. A tail clipping of each pup was taken at 1-2 weeks of age. DNA was extracted from tail clip samples using a Gentra Tissue DNA Extraction Kit, diluted and PCR amplified using primers for informative microsatellite markers. Multiple markers were tested spanning the length of the original line $\mathrm{T}$ locus from D2Rat194 to D2Rat201 (Table 1). Microsatellite markers were analyzed by PCR amplification using the GeneAmp Fast PCR system (Life Technologies) and separation on $3 \%$ high-resolution agarose gels in TBE buffer. SNP markers were analyzed by either PCR amplification followed by sequence analysis by the University of Louisville Center for Genetics \& Molecular Medicine DNA Core.

\section{Phenotype Analysis}

At 50-55 days of age nulliparous female homozygous WF and female congenic rats were administered DMBA (Acros, Pittsburgh, PA) at $65 \mathrm{mg} / \mathrm{kg}$ body weight by gavage. DMBA was suspended in sesame oil at a concentration of $20 \mathrm{mg} / \mathrm{ml}$, heated in 
Table 1:

Informative microsattelite markers used to narrow Mcs $1 \mathrm{~b}$ locus to $1.8 \mathrm{Mb}$ interval

\begin{tabular}{|c|c|c|c|c|}
\hline Samuelson Iab ID & Public ID & Position* & Forward Primer Sequence & Reverse Primer Sequence \\
\hline D2Rat 194 & D2Rat194 & $29237360-29237535$ & TA & GAGTGGATTTGAG $\wedge$ GCAGCTG \\
\hline $\mathrm{D} 2 \mathrm{Jwm} 17$ & D2Uwm17 & $32051319-3205163$ & АAGCTACAATGCCTAGCAAC & CСААCАGGACTTTAGTCATTG \\
\hline $\mathrm{D} 2 \mathrm{Mco} 43$ & $\mathrm{D} 2 \mathrm{Mco} 43$ & $32836549-32836763$ & AACCACTITIAGAATGTAATCAG & CGATCCTICATGGGGCTAACACT \\
\hline D2Gotl1 & D2Got11 & $33838802-33839075$ & CCTGGTCTCTGTCTCTGTCTCA & TCCTTTAGCCTTCCTTTTGG \\
\hline D2Rat 195 & D2Rat 195 & $33957192-33957523$ & TTGCTGTTTCTAGTATGTGCAGG & CCC $\triangle T G C A C A C A \Lambda G T A T G \Lambda A$ \\
\hline D2Rat 12 & D2Rat 12 & $39101957-39102109$ & CСАGTCCCTC $\triangle A \triangle A G G A \Lambda C A$ & GCA $A C C \Lambda C A T T T T C \Lambda G \Lambda \Lambda T T G \Lambda$ \\
\hline $\mathrm{D} 2 \mathrm{Mgh} 2$ & $\mathrm{D} 2 \mathrm{Mgh} 2$ & $39829003-39829053$ & GANATGGGGAGTCAGAGAAGG & TTTCTTGTTTACCTCTGTCTGGG \\
\hline D2Rat 199 & D2Rat199 & $41032096-41032201$ & TCAGGTATCTCCTATGGGGG & GAGCGC TCA TTG CTC TCT CT \\
\hline D2Rat 142 & D2Rat142 & $42318356-42318464$ & CACAAATGCATGTGTGCCTT & CAAAGCCTTTGATTGTGCAA \\
\hline D2Rat 16 & D2Rat 16 & $43376467-43376635$ & CTGCATGTGTTAAATCATTAGTCA & АCTTCАCGATCC $\triangle$ TTCTGGG \\
\hline g2UL1-5 & $12324219 \dagger$ & $4.3485572-43485628$ & AGACAATCCCCCACAGACAT & GAGAAGGTGCATGTCCAAA \\
\hline g2UL2-27 & $12324060 \dagger$ & $44195286-44195382$ & TAAATGTGGTTTCCTTTGCT & TCAACGTAGCTGAAATTGTG \\
\hline g2UL2-29 & NA & $44325512-44325564$ & CATAACAGCAAGAAGCATCA & GGAAAAGAACAACTGTTIGG \\
\hline D2Mco42 & D2Mco 42 & $45247893-45248161$ & GAGGAGTATATIAGTITGGGCTG & ATGGGCTGGCTAGTGAGAAAGIT \\
\hline g2UL2-30 & $12324220 \dagger$ & $44932096-44932309$ & ATTCAATTCCAACAATCCTC & САTTTTCAАGCCTTACАGGT \\
\hline D2Rat 200 & D2Rat200 & $48762858-48762979$ & AGGGTGGTTTGAAGCCAGTT & CAGGATTGAACAGCAAGCAG \\
\hline D2Rat201 & D2Rat201 & $49691463-49691646$ & GCAACCACAAAAGGAGAAGG & GCTAACTAGAATGCATTICAAAATT \\
\hline D2Rat202 & D2Rat202 & $51821000-51821161$ & TGGCTTAGCATAATCTCAGCA & CGCCCAGCTC $\triangle C A T T A \Lambda T T T$ \\
\hline
\end{tabular}

Abbreviations: WF, Wistar Furth; COP, Copenhagen

Genotypes determined using $3 \%$ high-resolution agarose gel

*Position based on Rattus norvegicus Chromosome 2, genome bulid version 3.4

† Marker IDs in NCBI database 
boiling water to dissolve and cooled to room temperature. For each congenic line, 15-25 female rats were used for phenotype analysis. At 15 weeks post DMBA administration, rats were euthanized and the total number of mammary carcinomas measuring $\geq 3 \times 3 \mathrm{~mm}$ in diameter were counted per rat. Spleens were removed to confirm genotype. Statview (SAS Institute, Cary, NC) statistical analysis software was utilized for analysis. Data are presented as means $\pm \mathrm{SD}$. Nonparametric Mann-Whitney tests were performed for analysis within congenic groups.

\section{Haplotype Block Analysis}

Human haplotype blocks and SNPs in LD are based on the GRCh37/hg 19 human genome assembly and were identified using the LD and Tagger functions in Haploview v.4.2 (www.broad.mit.edu/mpg/haploview/), respectively (Barrett et al., 2005). Sequences for human haplotype blocks were identified using the UCSC Genome Browser (genome.ucsc.edu/). Syntenic analysis was performed using the Convert function of the browser to determine the rat ortholog for the human haplotype block associated with each SNP (Kent et al., 2002).

\section{$\underline{\text { Results }}$}

\section{Line T Backcrosses Result in Novel WF.COP Mcs1b Congenic Rat Strains/Lines}

Mapping the $M c s 1 b$ QTL to a shorter syntenic interval reduces future comparative genetics work and abbreviates the list of potential candidate genes to be tested. To fine map the locus five congenic lines were generated by backcrossing $M c s 1 b$ line $\mathrm{T}$ and line B (Figure 2) to homozygous WF rats. These were termed F3, U2, W2, N3, and I4 
(Figure 4). Each of these lines contain a unique COP rat $\mathrm{Chr} 2$ segment of the $M c s 1 b$ candidate region from D2Uwm17:D2Rat201 (Chr2: 32051319 - 49691646) on a susceptible WF genetic background (Table 2, Figure 4). Lines W2 and U2 contained a COP allele spanning from marker $D 2 U w m 17$ to marker D2Rat 142 . Line N3 contained a COP allele spanning from marker $D 2 U w m 17$ to marker g2UL1-5. Line 14 contained a COP allele spanning from marker g2Uwm65-18 to marker $b U w m 15-3$.

When a unique recombinant animal was identified from a backcross, it was bred to a homozygous WF animal to generate additional heterozygous rats. Progeny were genotyped and pups lacking the recombinant allele were not used. Once enough male and female rats carrying the allele of interest had been generated they were inbred to produce homozygous rats. These homozygous rats were inbred from here forth to expand and maintain the line.

Mammary Carcinoma Susceptibility Phenotypes of Congenic Lines F3, U2, W2, I4 and N3 Shorten Mcs1b to a 2 Mb Interval

Mcs phenotypes were determined using tumor multiplicity at 15 weeks following DMBA induction of mammary carcinogenesis. As anticipated, the lines exhibited varying propensities to developing DMBA-induced mammary carcinomas. Rats homozygous for the F3, U2, and W2 COP allele developed $9.6 \pm 4.1(\mathrm{~N}=32), 5.7 \pm$ $3.9(\mathrm{~N}=6)$ and $6.0 \pm 1.8(\mathrm{~N}=18)$ tumors per rat, respectively, while littermate homozygous WF control rats developed $8.8 \pm 3.5(\mathrm{~N}=32), 6.3 \pm 2.2(\mathrm{~N}=12)$ and $5.9 \pm 3.2(\mathrm{~N}=9)$, respectively (Table 2). These numbers suggest that there is no difference between rats carrying the $\mathrm{U} 2$ and $\mathrm{W} 2 \mathrm{COP}$ alleles and rats having the WF allele in this interval. 


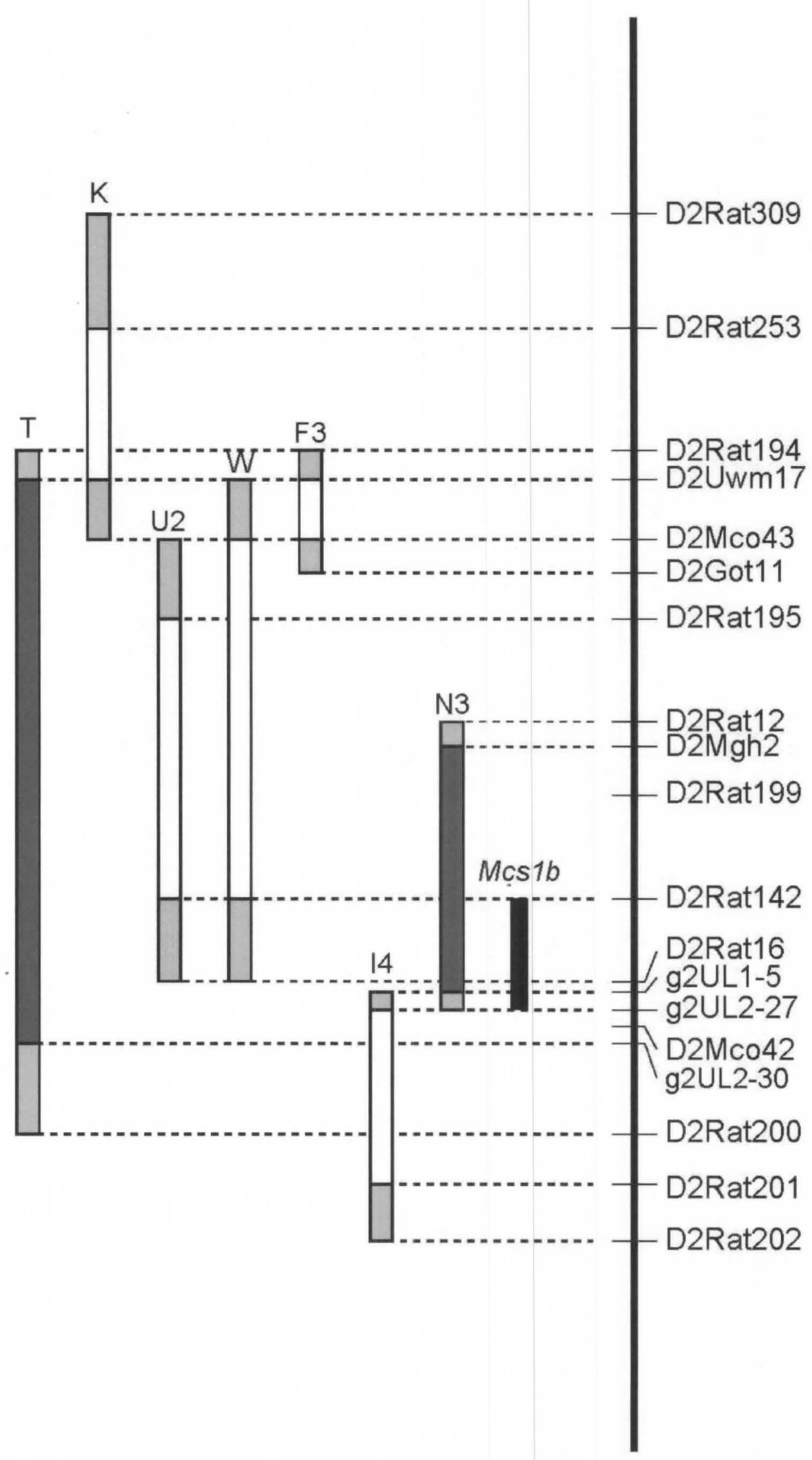


Figure 4: $\underline{\text { Rat } C h r ~} 2$ map of WF.COP lines delimiting $M c s 1 b$ to $1.8 \mathrm{Mb}$. Markers used to genotype WF.COP congenics are listed in relative positions on the $y$-axis. Lines are labeled with letter-number combinations and designated with filled dark-gray bars to indicate $M c s 1 b$-resistant alleles. Lines that are drawn with unfilled bars represent COP intervals incapable of conferring decreased susceptibility or resistance to mammary carcinoma development. The filled light-gray bars at ends of each congenic segment are intervals of unknown genotype. Lines $\mathrm{T}$ and $\mathrm{K}$ are shown again for reference. 
Table 2:

Mammary carcinoma multiplicity phenotypes (mean mammary carcinomas per rat \pm SD) by genotype for WF.COP Chr 2 congenic lines used to map Mes $1 \mathrm{~b}$ to $0.75 \mathrm{Mb}$

\begin{tabular}{|c|c|c|c|c|c|c|}
\hline WF.COP $C h r 2$ region & Line & $\begin{array}{l}\mathrm{COP} / \mathrm{COP} \\
(\mathrm{COP} / \mathrm{WF})\end{array}$ & $\mathrm{N}$ & $\mathrm{WF} / \mathrm{WF}$ & $\mathrm{N}$ & P value ${ }^{*}$ \\
\hline \multirow[t]{2}{*}{$\overline{D 2 U w m 17 / g 2 U L 2-30}$} & $\overline{\mathrm{T} \dagger}$ & $3.5 \pm 2.2$ & 21 & $8.3 \pm 3.3$ & 19 & 0.001 \\
\hline & $\mathbf{T} \dagger$ & $(7.6 \pm 3.4)$ & 18 & & & NS \\
\hline D2Uwm17/D2Ulb4 & F3 & $9.6 \pm 4.1$ & 32 & $8.8 \pm 3.5$ & 32 & 0.8433 \\
\hline \multirow[t]{2}{*}{$D 2 M g h 2 / g 2 U L 1-5$} & N3 & $3.4 \pm 2.0$ & 25 & $7.8 \pm 3.1$ & 25 & 0.0001 \\
\hline & N3 & $(5.5 \pm 3.6)$ & 15 & & & 0.0413 \\
\hline D2Ulb4/ENSRNOSNP 274 & W2 & $6.0 \pm 1.8$ & 18 & $5.9 \pm 3.2$ & 9 & 0.8498 \\
\hline D2Rat1 16/ENSRNOSNP27 & U2 & $5.7 \pm 3.9$ & 6 & $6.3 \pm 3.3$ & 12 & 0.8866 \\
\hline g2UL2-27/D2Rat201 & I4 & $9.3 \pm 3.0$ & 19 & $7.9 \pm 3.7$ & 13 & 0.247 \\
\hline D2Rat16/g2UL2-30 & $1 b-11$ & $5.9 \pm 2.2$ & 12 & $6.4 \pm 1.9$ & 8 & 0.4179 \\
\hline
\end{tabular}

Abbreviations: WF, Wistar Furth; COP, Copenhagen; Chr, chromosome

"P values from Mann Whitney tests

$\dagger$ Line T phenotype published previously by Haag et al. Cancer Research, 63:5808-5812, 2003 
Similarly, female rats homozygous for the I4 COP allele developed $9.3 \pm 3.0(\mathrm{~N}=19)$ tumors per rat compared to $7.9 \pm 3.7(\mathrm{~N}=13)$ for homozygous WF littermate controls again suggesting that the congenic I4 rats have the same mammary carcinoma susceptibility phenotype as homozygous WF rats. Conversely, female rats from line N3 that were homozygous for the $\mathrm{N} 3 \mathrm{COP}$ allele developed $3.4 \pm 2.0$ tumors $(\mathrm{N}=25)$ per rat while rats homozygous for the WF allele developed $7.9 \pm 3.7(\mathrm{~N}=13)$ tumors per rat. This is a $\sim 56 \%$ reduction in tumor multiplicity for animals bearing the N3 COP allele and is similar to the $\sim 58 \%$ reduction exhibited by rats carrying the line $\mathrm{T}$ COP allele. Additionally, line N3 heterozygotes, only carrying one N3 COP allele, developed $5.5 \pm$ $3.6(\mathrm{~N}=15)$ tumors per animal suggesting that the $\mathrm{N} 3 \mathrm{COP}$ resistance allele exhibits no dominance over the WF susceptible allele.

I was unable to define a precise distal end to the $M c s I b$ interval. Comparison of microsatellite DNA and published rat SNPs located in the $0.66 \mathrm{Mb}$ of genomic sequence between the distal and proximal ends of lines N3 and I4 yielded no genetic variation between resistant COP and susceptible WF alleles (Tables 3 and 4). It is possible that polymorphisms between $\mathrm{WF}$ and $\mathrm{COP}$ exist in this $0.66 \mathrm{Mb}$ region and have yet to be found; therefore, there remains a potential area of recombination from marker g2UL1-5 to marker $g 2 U w m 65-18$. Taken together these data delineate the $M c s 1 b$ locus to a 1.7 $\mathrm{Mb}$ region spanning from marker D2Rat142 at position chr2:42318464 to marker g2UL2-29 at chr2: 44325564 .

Targeted Resequencing of the WF and COP McsIb Locus Reveals New Polymorphic Markers and Tightens the Mcs1b Interval 
Table 3:

Primer Sequences for Microsatellites in $0.66 \mathrm{Mb}$ region of Mcsib locus

\begin{tabular}{|c|c|c|c|c|c|c|}
\hline Samuelson Lab ID & Public ID & Ensembl ID & Forward Primer Sequence & Reverse Primer Sequence & Position* & Variant \\
\hline AU 046380 & AU046380 & AU046380 & GCCACCATTGTTATCTGACACA & CTCAGTGTGAGACCATGGTTCA & $43313765-43313989$ & $\mathrm{WF}=\mathrm{COP}^{+}$ \\
\hline g2UL,2-22 & 12324055 & & CAAGCAACTGAGGGAGATAC & AGAGGCAATCAATTTGAAGA & $43531384-43531546$ & WF COP+ \\
\hline g2LL2-13 & 12324048 & & GTGTATGTTTAGGGGGTGAA & CCCAAAGATATGGTGAAGAA & $43536766-43536909$ & $\mathrm{WF}-\mathrm{COP}+$ \\
\hline g2UL2-23 & 12324056 & & GGACACATGAGCCAGTATTT & CTTGGAGACGACTGAAACTC & $43541442-43541637$ & $\mathrm{WF}=\mathrm{COP}+$ \\
\hline g2UL2-1 & 12324044 & & TACACTTGAGCAAGGACACA & ACAAGCCTGTTGTTOTAGGT & $43598558-43598706$ & $W F \mathrm{COP}+$ \\
\hline D2RAT267 & D2RAT267 & & CGCAGGAGAAACGCCTTATA & CCTCACTTTTCATCACICC"TG & $43605867-43605989$ & $W F=C O P \dagger$ \\
\hline D2Rat254 & D2Rat254 & & AGCATGACCAAGACATTCCA & CGCCATGGAGAGAGATGCTA & $43624817-43625068$ & $\mathrm{WF}-\mathrm{COP}+$ \\
\hline g2UL2-14 & 12324049 & & CGCTCTCTCTCTCATACACAC & CCTAAGACACTGGCTGAGAC & $43659242-43659460$ & $W F-C O P \uparrow$ \\
\hline g21:1.2-2 & 12324054 & & GGAGGTTCATTATGTRGTG & 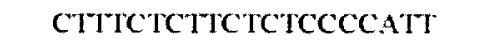 & $43697612-43697849$ & WF COP+ \\
\hline $\mathrm{g} 21112-15$ & 12324050 & & TTGGACCTAACACCTAGCAT & ATAGGTCGAGATGOAAAACA & $43704760-43704927$ & $\mathrm{WF}=\mathrm{COP}+$ \\
\hline g2UL2-16 & 12324051 & & GCCAGATGTAGTGGCATATT & GTTTGACTTTTGGGACAAAT & $43753048-43753283$ & $\mathrm{WF}=\mathrm{COP}+$ \\
\hline $\mathrm{g} 2 \mathrm{~L}$ & 12324061 & & GCAGGCAAAACATCTATACAC & ACTATGGGTAGAAACGCAAA & $43751704-43751942$ & $W F-C O P+$ \\
\hline g2II 2-24 & 12324057 & & CTTCCATACCTGACACACCT & AACATGACAGCTTTGGA AAT & $43840761-43841043$ & $\mathrm{WF} \mathrm{COP}+$ \\
\hline $\mathrm{g}^{2} \mathrm{UL} \cdot 2-4$ & 12324062 & & GTAGGGGCTACAAAGGAAGT & CCCAGGCTACACCATTATTA & $43845450-43845697$ & WF COPt \\
\hline $\mathrm{g} 2 \mathrm{UL.2-25}$ & 12324058 & & GGTOACTGGTGACAAAGATT & GTCAGCTTMGAAGGAACAC & $43911826-43912026$ & WF $\mathrm{COP}^{+}$ \\
\hline $\mathrm{g} 2 \mathrm{UL} 2-5$ & 12324063 & & TCTGGTTCAAAGAAGACCTG & AGGAGTTTTGGAGTGTCCTT & $43934522-43934761$ & $\mathrm{WF}=\mathrm{COP}+$ \\
\hline g2L12-6 & 12324064 & & AATCCCCTTCTCATTCTITC & GCATCTTAAGGTCTGGAGTG & $43935644-43935818$ & $\mathrm{WF} \mathrm{COP}+$ \\
\hline g2U1.2-7 & 12324065 & & TCTGTRCAGTGAGAGATCCA & AGTCAGGACAAAGATACACAAG & $43951932-43951951$ & $\mathrm{WF}=\mathrm{COP}+$ \\
\hline $\mathrm{g} 2 \mathrm{V1}, 2-26$ & 12324059 & & AGCAGCTGGTATAGAAAAGGT & GCACACTTGAGAGTGAGTGA & $43969019-43969339$ & $\mathrm{WF}=\mathrm{COP}+$ \\
\hline g2UL2-9 & 12324066 & & GGGAAGGAAAGACTGACTTC & AGTGCTTGTGATGTCCTCTC & $44024519-44024723$ & $\mathrm{WF}-\mathrm{COP}+$ \\
\hline g2L1.2-10 & 12324045 & & CGGATCTTTTGAGTTTGAAG & GTGGAATGGGGAAACTATTA & $44033098-44033281$ & $\mathrm{WF}=\mathrm{COP}+$ \\
\hline $\mathrm{g} 241 \mathrm{~L} 2-18$ & 12324052 & & TCGAGCTGTCTTCTGACTG & TTCTCCCTTCATCATGTCTC & $44089085-44089302$ & $\mathrm{WF}=\mathrm{COP}^{2+}$ \\
\hline g2UL2-11 & 12324046 & & CGCTAAACCTGTCAACCTAC & AATAAAACCCACCACACAAC & $44118131-44118361$ & $\mathrm{WF}=\mathrm{COP} \uparrow$ \\
\hline g2UL2-19 & 12324053 & & TTCCCACTTCTTCATTCCTA & GTCTGAACCCTACCATGAAA & $44128126-44128291$ & $W F=C O P+$ \\
\hline D2Rat298 & 12324043 & & TGCAACTATCTATGCCAGTTGG & CAGGACAGGCAGAAGATGCT & $44149293-44149181$ & $\mathrm{WF}=\mathrm{COP}+$ \\
\hline g2UL2-12 & 12324047 & & GAGGAGTCATCGACTGGTIA & CCTRGCTCTCTTTCAAATTC & 44164273.44164510 & $W F=C O P+$ \\
\hline g2UL2-27 & 12324060 & & TAAATGTGGTTRCCTTTGCT & TCAACGTAGCTGAAATTGTG & $44195286-44195382$ & $\mathrm{WF} \cdot \mathrm{COP}^{+}$ \\
\hline D2Got 27 & D2Got27 & & ACCGTATGCACTTGATTTACAGAT & CСATTAAGTTGTCCTCTGCCC & $44205860-44205910$ & WF $\times$ COP + \\
\hline
\end{tabular}

BN, Brown Nonway; COP, Copenhagen; WF, Wistar Furth

* Position is Rattus nonegicus.s genome build version 3.4

+ Microsatellite DNA genotypes were determined using $3{ }^{\circ} \%$ high resolution agarose 
Table 4:

SNP markers tested on WF and COP rats based on published datidof SNPs in the Mos 1 b interval

\begin{tabular}{|c|c|c|c|c|c|c|c|c|}
\hline Samuelson lab ID & Publicid & Ensemble ID & Forward Primer Sequence & Reverse Primer Sequence & Position* & $\begin{array}{c}\mathrm{BN} \\
\text { (reference) }\end{array}$ & COP & WF \\
\hline RN02UL $A 12 \pi$ & & & AAAATGCAGACCCATGTGC & TGATGCCCTTTCATTGTCC & 42142131 & $c$ & $\mathrm{c}$ & $T$ \\
\hline RNO21 I,-A12u & & & GCAAAGMTCATCTOTTRT: & GCTACAACACACACACGTCAA & 42170174 & c & c & $A$ \\
\hline RNU21:1-A12v+ & $r \times 63769957$ & ENSRNOSNP2740854 & GACOKGTAGTOKGAA & MGGAOCALATGGCTCAGA & 42364155 & $A$ & $\mathrm{~A}$ & $c$ \\
\hline RNo2UL-A12u & $\mathrm{rs} 64618233$ & & AGTCCCTCACAATOGTAGAA & GOCCAGGAATAATGAGCAA & 42421698 & $A$ & G & G \\
\hline RNo2L I I A $12 \mathrm{x}$ & กs64418578 & FNSRNOSNP2785536 & MTGATTGGCCCCAGTGT & MTG ANCTCATCGGCCTCT & 12514359 & $\therefore$ & G & G \\
\hline RNO21TI-A12Y & $\mathrm{rs} 105808796$ & FNSRNOSNP I 382723 & OCCTTGCTOTTTACATCACC & CAOC AATTACCGAGATGCAC & 42633031 & $\mathrm{~s}$ & $\therefore$ & A \\
\hline RNo21 L.-A12z & $\operatorname{rsc6} 6162544$ & ENSRNUSNP2785538 & TOCATEOTACOATOCATE & AGCTATCCACTEAATICTC & 42679006 & 6 & $\mathrm{~A}$ & $A$ \\
\hline RNO2LL-A12aa & rs 105703830 & LNSRNOSNP1382993 & CTGTACCATITCTGCCATGC & CATCATTGAATTTTGAGAAGCA & 42782209 & $A$ & $\mathbf{T}$ & $\mathbf{T}$ \\
\hline $\mathrm{RN} 02 \mathrm{t} \mathrm{L}-\mathrm{A} 12 \mathrm{bb}$ & rs 105836290 & ENSRNOSNP1383048 & COGNGCTIGCTIGTTTGTT & TOGAGACCCACGCTCTI & 42822772 & $\mathbf{T}$ & c & c \\
\hline RNo2L1- $+12 \mathrm{ec}$ & r\$63938035 & FNSRNOSNP278554! & GCCTATGATGTAATOCCCAOT & CACATCTGTGATCGCATAGC & 42865664 & $\mathbf{T}$ & c & $c$ \\
\hline RNO2LLLAJ2dd & $r \leqslant 107042005$ & LNSRNUSN12785542 & GGCATCITGGACGRGA & TIGGATGGGGCAAAGACTA & 43135289 & $A$ & (; & G \\
\hline RNO2UL-A120e & 18106693272 & LNSRNOSNP2785543 & MTCTGGOATTGAGGACOA & CAAGCCGATCTCACCAGIT & 43252585 & c & $\mathbf{T}$ & $r$ \\
\hline$R N 021 \mathrm{~L}-A 12 \mathrm{I}$ & r\$64339116 & ENSRNOSNP1383915 & CTOCCAGAAGCATCTAAAAACT & AAGTGGOTGCTCTGTCITCA & 43523570 & $\mathrm{~T}$ & $c$ & $c$ \\
\hline RN02UL-N12g & Is 107369228 & ENSRNOSNP2785545 & CTAATTGTT TCTGCTR & ATCCTATCCANCTCTI & 43544727 & c & c & $c$ \\
\hline $\mathrm{RN0211}-\mathrm{A} 12 \mathrm{hh}$ & rs66091881 & FNSRNOSNP1384088 & TGC AAACTGTTACAAAG & CCATGATTACTCCTTTT & 43660982 & $\mathrm{~T}$ & c & $c$ \\
\hline RNO2I L-A12ii & $\mathrm{rs} 65824840$ & ENSKNOSNP2718470 & TIAGACAGAACCOTC & CTGACTAGTTICET & 43699296 & $A$ & $a$ & ( \\
\hline RNO2LI.-A12ii & $\mathrm{r} 8143555$ & & CCAGGAAATTAACACTT & GCTCCATGATACATCC & 43836007 & c & $\mathbf{T}$ & $\mathbf{T}$ \\
\hline RN02KL L $112 \mathrm{kk}$ & 188171129 & ENSRNOSNP2785549 & AC. MANGTCTRCTCAAC & GTCCCATOTTTCTAGTTA & 43838288 & c & $\mathrm{T}$ & $\mathrm{T}$ \\
\hline $\mathrm{RNO} 2 \mathrm{I} \mathrm{I}-\mathrm{A} 12 \mathrm{n}$ & $\pi 107117602$ & FNSRNOSNP2785550 & CITAGCAACATACCTTCT & CRACGATGAGTTGTCTTT & 43918079 & G & $A$ & $A$ \\
\hline RNo2UL-A12mm & 1565844585 & ENS1RNOSNP1 384598 & CATCATTAGICACTGCT & CCAACAGTTMAGTTICT & 43987419 & G & $A$ & $A$ \\
\hline $\mathrm{RN} 021 \mathrm{H}-\mathrm{A} 12 \mathrm{nn}$ & rs 66189322 & ENSRNOSNP273129? & GATGCATGGTATATACTOOT & GATCTTAAANCCATRAG & 44180660 & $c$ & c & $c$ \\
\hline $\mathrm{RN} 021 \mathrm{~L}_{-} \mathrm{A1200^{+ }}$ & $\mathrm{ss} 107066908$ & ENSRNOSNP2785553 & GCATTGACACTTGTTTA & AGCTATCTGTATTGGTTC & 44210175 & c & $\mathrm{c}$ & $\mathrm{G}$ \\
\hline $\mathrm{RN} 02 \mathrm{UL}-\mathrm{A} 12 \mathrm{pp}$ & rs64021638 & ENSRNOSNP1384967 & COTGGTTGTATTCTANT & CAGTAGTCACTCCACATT & 44242105 & c & $\mathrm{T}$ & $\mathrm{T}$ \\
\hline
\end{tabular}


Table 4 continuted

\begin{tabular}{|c|c|c|c|c|c|c|c|c|}
\hline Samuelson tab ID & Publicil & Linsemble $1 \mathrm{D}$ & lorward Primer Sequence & Reverse Primer Sequence & Position* & $\begin{array}{c}\text { BN } \\
\text { (reference) }\end{array}$ & $\mathrm{COP}$ & WI: \\
\hline A48-ENSRNOSNP-1383684 & $n 105214022$ & ENSRNOSNP1383684 & AGCCTGCACTGCCAACGTA & TGCTGGATGCACCGCTAC & 43294777 & $\mathrm{c}$ & $\mathrm{T}$ & $T$ \\
\hline 448-FNSRNOSNP-1.383696 & rs 106710684 & FNSR YOSNP1383696 & CTCTCCGGCAGTCACAGGTT & TGGAGACTGGGAATGCAAATG & 43308266 & $\mathrm{~T}$ & c & c \\
\hline A48-IENSRNOSNP-13838\% & $\mathrm{rs} 6.4839173$ & ENSRNOSNP1383896 & TCAGGGCGAAC TGAGAAAG & GCOLCACACGAACATCTEC & 43507047 & G & $A$ & A \\
\hline A48-ENSRNOSNP-1384351 & rs64812820 & ENSRNOSNP1384351 & GAACCCCTTCCTCGCTTGAT & TGTGGCTGTGGGTCACCA & 43843704 & $A$ & $G$ & G \\
\hline A48-ENSRNOSNP-1384874 & $\mathrm{rs6} 5209285$ & ENSRNOSNP1384874 & CTGTGCCAGCAGGCTAGGT & AGCCTAGGTGGATGTGATGA & 44159855 & c & $\mathrm{T}$ & $\mathrm{T}$ \\
\hline 448-ENSRNOSNP- 1384880 & $\mathrm{rs} 66201784$ & ENSRNOSNPI 384880 & CATGTCOTGCTTCCTGTCCTT & TCAGGAAGTCATOGAGGTGAGAC & 44164060 & $A$ & G & c \\
\hline A48-ENSRNOSNP-2645369 & N107488981 & ENSRNOSNP2645369 & TICAAAATGGCGGAOTGCTT & TCTOGGAGTTAGTOCIOTCTG & 42712802 & $\mathrm{~T}$ & c & $\mathrm{c}$ \\
\hline A48-ENSRNOSNP-2654615 & $\mathrm{rs64619323}$ & ENSRNOSNP2654615 & MTACAGGCGGTGGATATGANOA & AGCCTGGTCCAGTGTCTC UTC & 43669623 & 1 & G & $\mathrm{G}$ \\
\hline A48-FNSRNOSNP-2657379 & $\mathrm{rs65363226}$ & FVIRVOSNP2657370 & 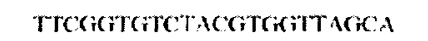 & COTCCAAATATCTOCATITACTIC & 43815443 & $A$ & ci & G \\
\hline A48-1ENSRNOSNP-2685221 & $\operatorname{rs660162549}$ & R.VSRNOSAP2685221 & CATRGCTACETATCCACTIKGGA & GACOTGAOCOCATACAAAC & 42679006 & G & $A$ & $A$ \\
\hline A48-ENSRNOSNP-2686459 & 1s63770151 & ENSRVOSNP2686459 & CAGTGTCTCCACCGACTGACC & CTITGCCCCGATACATCCAC & 43529896 & $c$ & $\mathrm{~T}$ & $\mathrm{r}$ \\
\hline A48-FNSRNOSNP-2705085 & $\operatorname{rs} 6546.3498$ & FNSRNOSNP270508S & AGTGECOCTACCTCAACA & GGCCATTGAGTCGCTCGOTA & 4.3418586 & c & $\mathrm{r}$ & $\mathrm{T}$ \\
\hline A48-ENSRNOSNP-2706005 & $\mathrm{rs} 66268295$ & ENSRNOSNP270G00S & GGAGCTAAGCGQTGTGAGGA & AGCOTRCAAGGATGGGGAAG & 43517914 & $\mathbf{G}$ & $A$ & $A$ \\
\hline A48-ENSRNOSNP-2706295 & rs66174360 & EVSRNOSNP2706295 & AATCCCCAACATCCCTTCCT & CATGCCACACCCAAACATTG & 43377935 & $A$ & $A$ & $A$ \\
\hline A48-ENSRNOS.NP-2708093 & $\mathrm{rs6} \$ 540697$ & FNSRNOSNP2708093 & TAGGCACCGAGAAGCCACAT & AGGGACTCCTGGAAGGGATG & 43418057 & G & c & $c$ \\
\hline 148-ENSRNOSNP-2744110 & $\approx 64996.390$ & ENSR NOSNP2744110 & AOCATGCCOTGACTC RGAAT & CAABGETICTEGITETRCA & $4300601 ?$ & c & $\mathrm{T}$ & $\mathrm{r}$ \\
\hline A48-ENSRNOSNP-2769457 & $r 863803615$ & ENSRNOSNP2769457 & AAGAGICCAGGLAGGGLGGA & GCACTAAGCGATACCCATGA & 43376687 & $c$ & $c$ & $c$ \\
\hline A48-ENSRNOSNP-2753529 & ts64232116 & ENSRNOSNP2753529 & TCITGGGTACACTGCCCACA & ACGGNGGGTTTGCCTGITT & 43753769 & 1 & $\mathrm{~T}$ & $\mathrm{~T}$ \\
\hline A48.FNSRNOSNP-2778924 & $\mathrm{r} \leqslant 106934580$ & FVSR NOSNP2778924 & ACTGGCTTGGCTCTTCOTGT & TGCTGGGCTAAAAGGGAGGT & 43191996 & $\mathrm{~T}$ & c & c \\
\hline
\end{tabular}

Abbreviations: BN. Brown Norway (reference) COP, Copenhagen; W1, Wistar liurth

* Position is based on Rattus nomegicus. Chromosome 2, genome build version 3.4

† Informative markers used in narrowin(iMcslb locus

Sources: RN021L markers. Nijman eTal. RMICGenomics 2008: A48 markers. STAR Constorium database 
Up to this point, all mapping had been performed using microsatellite markers. SNPs between WF and COP have not been tested and new markers were needed in the region to effectively fine-map the $M c s 1 b$ locus. I resequenced targeted regions in the newly delineated Mcs1b interval from D2Rat142 to g2Uwm65-18. Cuppen and colleagues published a panel of SNP markers for the rat genome consisting of 820 different SNP assays tested in 34 different rat strains (Nijman et al., 2008). SNPs assayed in the study were chosen based on being polymorphic between BN, Wistar and Dahl/Salt Sensitive (SS) rats. Of the 820 SNPs tested, 22 lie within the $M c s l b$ locus, making this a good tool to identify polymorphisms between WF and COP alleles (Table 4). Although the Cuppen panel was tested on 34 different rat lines, the COP strain was not included. Further, WF rats were tested but they were not the Harlan (WF/Hsd) strain used in my study. It was therefore, necessary to resequence WF and COP gDNA at these 22 sites to determine if they were polymorphic.

Additionally, a rat SNP map has been developed by the Specific Targeted Research Project (STAR) Consortium (Saar et al., 2008). The consortium sequenced $>100,000$ SNPs in 60 different rat strains, including COP/Hsd; however, WF/Hsd was not included. To identify potential polymorphisms between WF and COP sequences I identified 20 non-redundant SNPs from the STAR Consortium database lying within the rat Mcs $I b$ locus (Table 4). These were tested in WF and COP gDNA samples by Sanger sequencing.

Of the 22 Cuppen SNPs tested, 4 were identified to be polymorphic between WF and COP alleles. These were termed $A 12 t, A 12 u, A 12 v$, and $A 12 o o$. Markers $A 12 t, A 12 u$, and $A 12 v$ lie relatively close to each other at positions $c h r 2: 42142131, c h r 2: 42170174$ 
and chr2:42364155 (Figure 5). Marker A12oo, however, lies much further downstream near the proximal end of line I4 at position chr2:44210175 (Figure 5). Sequence results of SNPs from the STAR Consortium database revealed no polymorphisms between WF and COP at any of these markers. All newly-identified markers will be useful in future mapping studies to narrow down the $M c s 1 b$ locus.

I also attempted to identify new microsatellite markers by amplifying regions of sequence containing di- and tri-nucleotide repeats, which are typical of microsatellite polymorphisms. Results of these studies uncovered no polymorphisms between WF and COP sequences. In addition, large regions of gDNA were resequenced in these areas across the Mcs Ib interval to identify potential SNPs. The results of this study showed no polymorphisms between the two rat strains (Table 5).

The new SNP markers were tested on rats from congenic lines U2, W2, N3 and I4. Lines $\mathrm{U} 2$ and $\mathrm{W} 2$ tested homozygous for the COP allele at $A 12 v$ and homozygous for the WF allele at the more distal SNPs. Line I4 tested homozygous for the COP allele at A120o and homozygous for the WF allele at the proximal SNPs. Line N3 tested homozygous for the WF allele at a12oo. Taken together, these markers slightly shorten the $M c s 1 b$ interval to a region from $A 12 v$ to $A 12 o o(\mathrm{Chr} 2: 42364155-44210175)$.

\section{Backcrossing Line T Generates a Novel WF.COP Congenic Line: 1b-11}

There are 10 annotated transcripts that lie in the newly-defined $M c s l b$ locus (Figure 5). Also, there is a region of potential recombination between markers g2UL1-5 and $A 1200$. Further mapping could eliminate some of these transcripts and reduce the number of potential gene candidates that need to be tested. To do this, I attempted to 


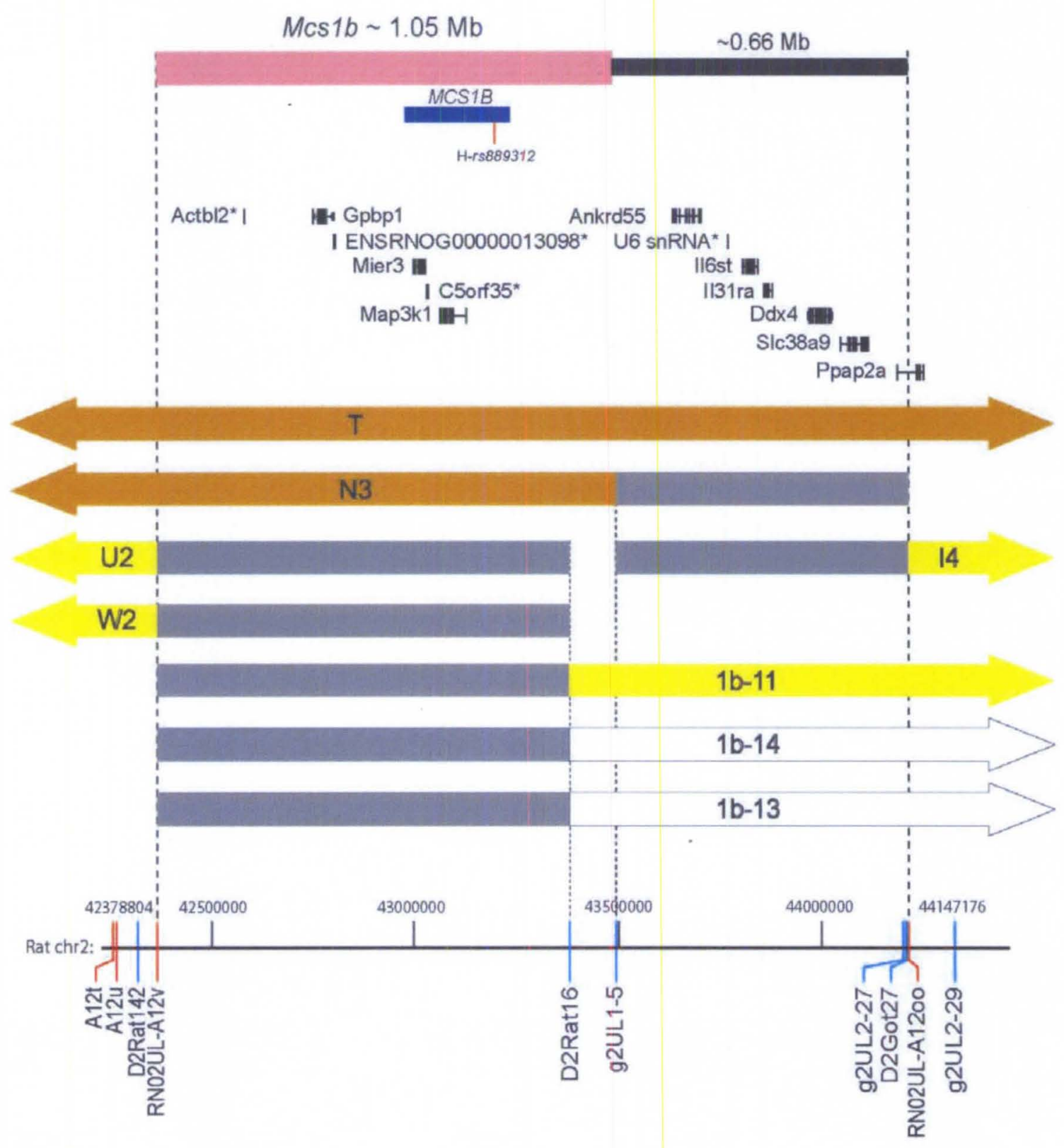


Figure 5: Genetic landscape of fine-mapped rat Mcs $1 b$ locus. $\square$ and $\square$ represent regions with a COP genotype with resistant and susceptible Mcs phenotypes, respectively. $\square$ represents regions with the COP genotype where the Mcs phenotype is not known. $\square$ denotes regions of potential recombination where no markers are currently known. Red and blue lines demarcate the location of informative SNP and STS markers used to genotype the locus. The blue bar depicts the rat interval orthologous to the human breast cancer-associated locus; SNP rs889312 and SNPs in LD are shown. Annotated transcripts obtained from UCSC Genome database shown in black. 


\section{Table 5:}

$\underline{\text { Regions sequenced between Rat } \mathrm{Chr} 2: 43125940 \text { and } \mathrm{Chr} 2: 43632545 \text { to identify potential }}$ polymorphisms between WF and COP in the $M c s 1 b$ locus

Total conserved regions attempted to sequence 194

Regions Successfully Sequenced

Regions successfully sequenced with results that match reference gDNA files

$\%$ Regions sequenced resulting in both WF and COP sequences matching reference

$\%$ Regions sequenced resulting in either WF or COP sequences matching reference

Total potential polymorphic regions identified

Potential Microsatellites

Potential Insertion/Deletions 
generate a new congenic line containing a COP allele from the end of line N3 at microsatellite marker g2UL1-5 and spanning marker $A 120 o$ and beyond. I backcrossed line $\mathrm{T}$ heterozygous rats to homozygous WF rats to generate novel unique recombinants containing this region. 268 progeny were tested from these crosses and generated 3 new recombinants. These recombinant animals contained COP alleles spanning markers g2UL1-5 to g2UL2-30 and were termed lines $1 \mathrm{~b}-11,1 \mathrm{~b}-13$, and 1b-14 (Figure 5). These recombinants were backcrossed to expand the population and progeny were genotyped. Pups containing the recombinant COP allele were inbred to establish a homozygous fixed congenic line. Due to complications with attaining recombinant pups and insufficient litter sizes, only $1 \mathrm{~b}-11$ was established as a new congenic line. This line was subsequently phenotyped as described.

Mammary Carcinoma Susceptibility Phenotype of WF.COP Congenic Line 1b-11 Is Not Different Than Homozygous WF Controls

Female rats homozygous for the $1 \mathrm{~b}-11 \mathrm{COP}$ allele and homozygous WF controls were administered DMBA at 50-55 days of age and euthanized at 15 weeks post-DMBA administration. Female $1 \mathrm{~b}-11$ rats developed $5.9 \pm 2.5(\mathrm{~N}=12)$ tumors per rat compared to $6.4 \pm 1.9(\mathrm{~N}=8)$ tumors for WF controls (Table 2, Figure 6). The difference in tumor multiplicity between the two lines is not statistically significant $(\mathrm{P}=0.4179)$. These data suggest that there is no difference in the mammary carcinoma susceptibility phenotype between $1 b-11$ congenic rats and WF rats.

\section{WF.BN Congenic Rat Strain/Line Is Resistant To Developing Mammary Tumors}




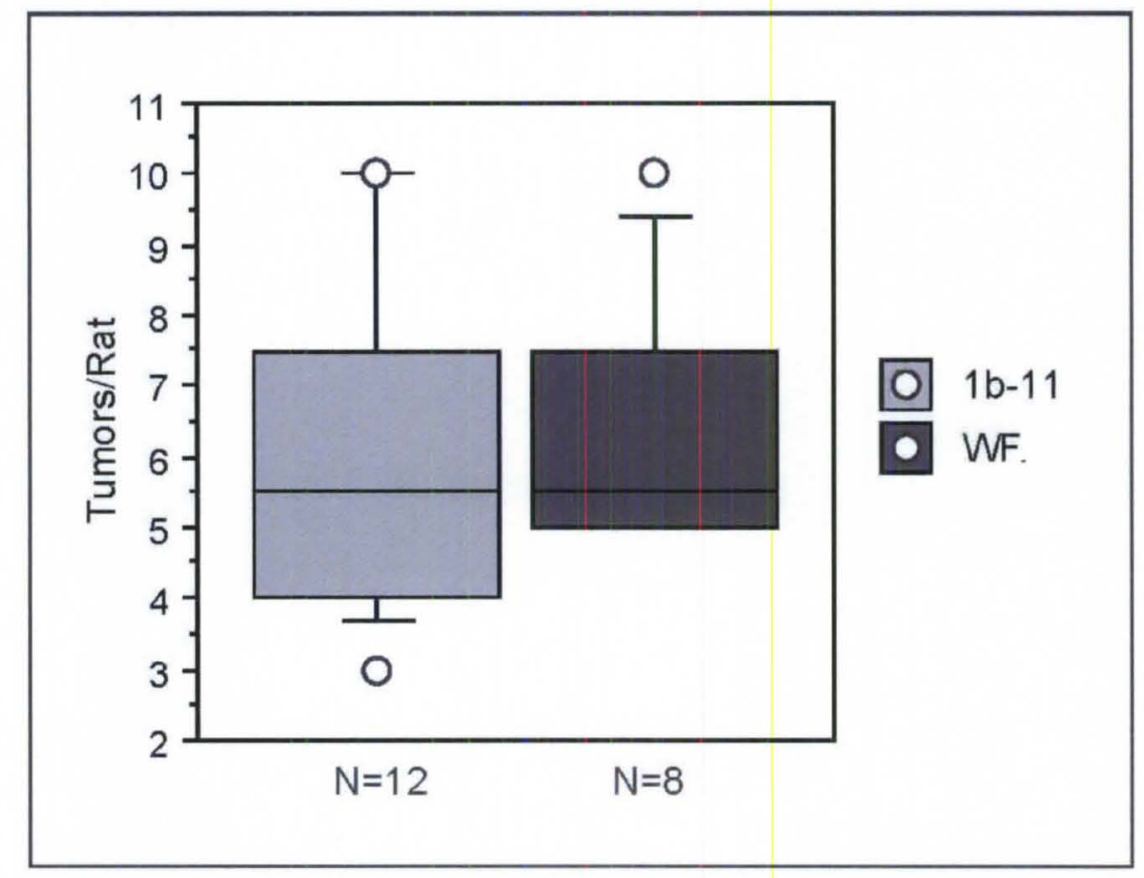

Figure 6: Endogenous mammary tumors formed in congenic line $1 \mathrm{~b}-11$ and homozygous WF animals. Tumors presented as box plots. Circles represent outlier datapoints. $\mathrm{N}$ value represents number of rats tested. 
$\mathrm{BN}$ rats are almost completely resistant to chemical, radiation and oncogeneinduced mammary carcinogenesis. The Mcslb locus was identified between WF and $\mathrm{COP}$ rat strains and it is not known whether this locus is functioning in the $\mathrm{BN}$ rat to reduce mammary carcinoma susceptibility. If it is, the $\mathrm{BN}$ rat may be another useful strain to study the Mcs $1 b$ QTL.

To address this, I used a WF.BN congenic line. WF.BN congenic rats were developed by introgressing a $\mathrm{BN}$ allele at the $M c s 1 b$ locus onto a WF background using the method described previously. Female WF.BN N4F1 homozygous rats were phenotyped along with homozygous WF controls. WF.BN N4F1 homozygous rats developed $2.3 \pm 1.5(\mathrm{~N}=11)$ tumors per rat while WF controls developed $5.4 \pm 2.6$ $(\mathrm{N}=28)$ tumors per rat (Figure $7 \mathrm{~A})$. The difference between these means is significant $(\mathrm{P}=0.0012)$ and it suggests that the mammary carcinoma resistance exhibited by the $\mathrm{BN}$ rat strain may be partly conferred by heritable elements lying within the $M c s 1 b$ locus. To be certain there was no difference between WF.BN N4F1 rats carrying a WF allele at $M c s 1 b$ and inbred homozygous WF rats, these groups were also compared (Figure 7B). There is no statistical difference $(\mathrm{P}=0.2039)$ between congenic WF.BN N4F1 rats homozygous for the WF allele at Mcslb and inbred WF rats; therefore, these could be pooled as susceptible controls.

With the knowledge that there are two other Mcs loci on rat chromosome 2, it was necessary to define the full interval of the BN allele. To determine the length of the $M c s I$ locus within the WF.BN locus, the N4F1 rats were genotyped at markers along the original Mcs1 locus (Figure 2, line B; Table 1). WF.BN N4F1 rats had a BN allele spanning markers D2Uwm17 to D2Rat210 (chr2:32051319-82193231) delineating a 50 
A

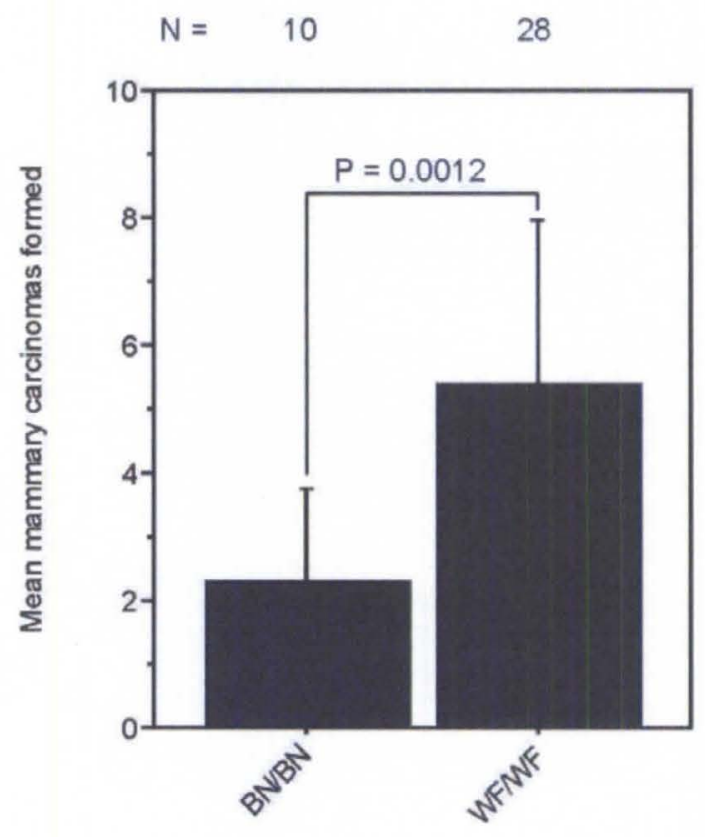

B

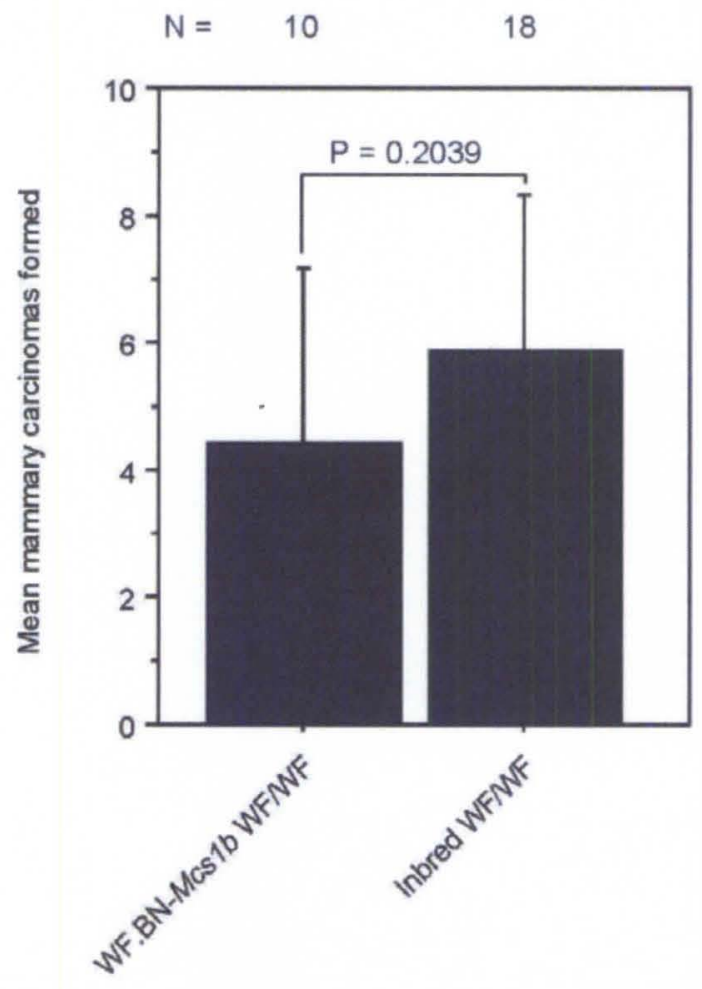

C

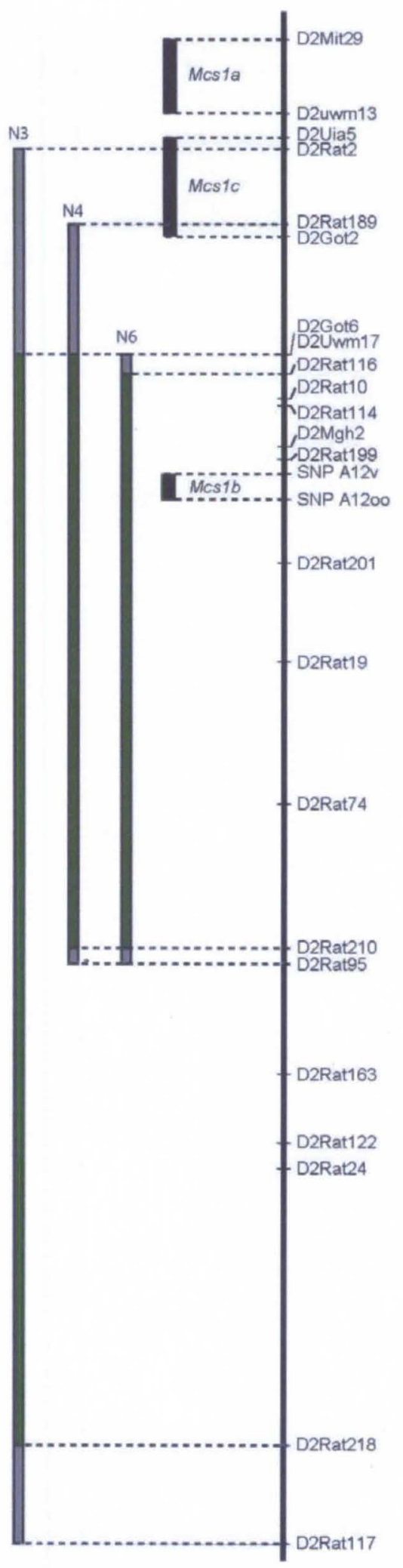


Figure 7: Tumor multiplicity in WF.BN congenic lines. A; Average mammary carcinomas formed in congenic WF.BN N4F1 and homozygous WF control female rats after being administered DMBA. Error bars denote standard deviation. Data was analyzed using Mann Whitney test. B; Average mammary carcinomas formed in congenic WF.BN female rats compared to inbred WF female rats. C; Map of WF.BN congenic lines at N4 and N6 generations. $\square$ denotes a BN genotype with a resistant Mcs phenotype. $\square$ denotes regions of potential recombination. Black bars designate Mcs loci 1a, 1b, and 1c previously identified by Haag et al. 
$\mathrm{Mb}$ region (Figure $7 \mathrm{C}$ ). There is a region of potential recombination at the proximal end of the interval that overlaps with the $M c s l c$ locus; therefore, the $M c s l c$ locus may be contributing to mammary carcinoma resistance in this line. Thus, I again attempted to reduce the interval by backcrossing the WF.BN congenic rats to WF rats up to the N6 generation and these animals were again genotyped to identify the ends of BN allele. The N6 generation contained the $\mathrm{BN}$ allele spanning markers D2Rat116 to D2Rat210 (chr2:33845498-82193231) shortening the interval to $\sim 48 \mathrm{Mb}$ and eliminating overlap with the Mcs Ic locus. I attempted to further breed these animals out to the N10 generation to eliminate the long stretches of $\mathrm{BN}$ genomic sequence flanking the $M c s 1 b$ locus; however, due to complications, the WF.BN congenic line was lost. It was decided that it was not feasible to pursue reestablishing the WF.BN congenic line.

\section{$\underline{\text { Discussion }}$}

The $M c s 1 b$ locus was previously delineated to $\mathrm{a} \sim 15 \mathrm{Mb}$ interval bounded by markers D2Uwm17 and g2UL2-30 on rat chromosome 2 (Haag et al., 2003)(Figure). More than 50 transcripts lie in this region. To shorten this list and reduce subsequent comparative genetics work I sought to fine-map this locus using a combination of WF.COP and WF.BN congenic rats.

Five congenic lines were developed using a similar approach to what was used previously to identify the McsI and its subloci: Mcs Ia, b and $c$. Lines F3, U2, W2, I4 and N3 all contain COP alleles spanning various portions of the original line $\mathrm{T}$ background genome (Figure 4). Testing these lines for mammary carcinoma susceptibility enabled me to narrow the $M c s l b$ locus. The tumor multiplicity data 


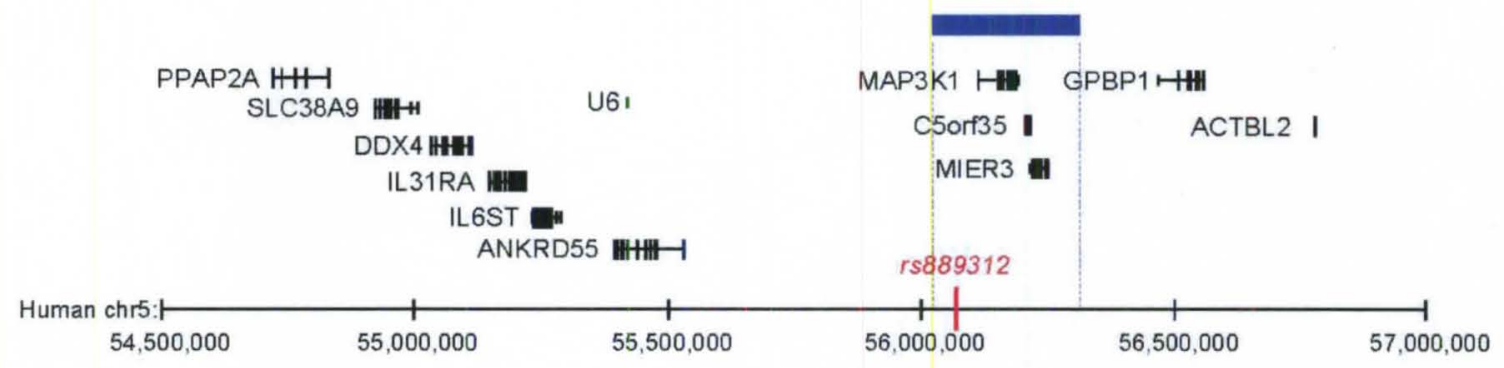

Figure 8: Map of human Mcs $1 b$ orthologous locus on human chromosome 5. Base positions are labeled at the bottom moving proximal to distal from left to right. The blue bar denotes $280 \mathrm{~kb}$ haplotype block containing SNP rs889312. The genomic position of SNP $r s 889312$ is shown in red. Transcripts are shown with exons designated by vertical bars. 
obtained from lines $\mathrm{U} 2, \mathrm{~W} 2, \mathrm{I} 4$ and N3 delineate the $M c s 1 b$ locus to a $\sim 1.8 \mathrm{Mb}$ interval on chromosome 2 between markers $A 12 v$ and $A 120 o$. This markedly reduces the number of transcripts to be functionally tested to thirteen.

As anticipated, the rat ortholog to the human breast cancer-associated $280 \mathrm{~kb}$ haplotype block lies within this $1.8 \mathrm{Mb}$ region. It is likely that a heritable element(s) lying in this region is controlling rat mammary carcinoma susceptibility in a manner potentially analogous to the human orthologous locus. Therefore, the rat may be used to identify the mechanism of action and translate this to human disease susceptibility. The human block contains 2 known transcripts, $M I E R 3$ and $M A P 3 K 1$, as well as a predicted gene transcript, C5ORF35 (Figure 3). Nearby, but lying outside of the human haplotype block associated with $r s 889312$, are approximately 7 other annotated transcripts (Figure 8). The rat contains orthologs to these transcripts within the $M c s l b$ locus although the orientation of the rat $M c s 1 b$ locus is reverse of the human locus (Figure 5). I anticipate that one or a combination of the three transcripts lying inside the human haplotype ortholog is causative for the mammary carcinoma susceptibility phenotype. However, all of the transcripts within the $M c s 1 b$ locus, including those lying outside the human haplotype block orthologous interval, must be tested as they cannot be ruled out as causal.

The Mcs $1 b$ locus was mapped with the use of polymorphic microsatellite markers published in the RGD public database (Dwinell et al., 2009). However, many of these were not informative between WF and COP genotypes. Targeted resequencing of regions in the narrowed $M c s I b$ locus uncovered 4 SNPs polymorphic between the WF and COP strains. Using these new SNP markers, the Mcs $1 b$ locus was mapped to a 
shorter interval between markers $A 12 v$ and $A 120 o$. Also, short genomic regions rich in di- and tri-nucelotide repeats within the $M c s 1 b$ interval were sequenced; however, no polymorphisms were identified between WF and COP alleles. Taken together these data suggest that WF and COP share similar haplotypes. However, since these newly identified SNPs are positioned at the ends of the Mcslb interval, it is unlikely that they are causative; therefore, more polymorphisms likely exist in this region.

Breeding of line $\mathrm{T}$ led to the development of another congenic line, 1b-11. Line $1 \mathrm{~b}-11$ has a COP genotype from marker D2Rat16 to marker g2UL2-30. This interval crosses the $0.66 \mathrm{Mb}$ region from $g 2 U L 1-5$ to A12oo making it useful in identifying if this area of potential recombination is contributing to the resistance phenotype (Figure 5). Tumor multiplicity between homozygous $1 \mathrm{~b}-11$ female rats and homozygous WF littermates was not statistically different (Table 2 ). These data imply that this 0.66 region from D2Rat16 to A12oo does not contain the element responsible for the Mcs $1 b$ phenotype. It stands to reason, then, that the element conferring resistance to mammary carcinoma development resides in the interval from $A 12 v$ to $D 2 R a t 16$, thereby narrowing the Mcslb locus to $\sim 1.01 \mathrm{Mb}$.

These data signify that a more complex mechanism may be at work. Tumor multiplicity data for line I4 and WF littermate control rats was $9.3 \pm 3.0$ and $7.9 \pm 3.7$, respectively. Although the difference is not significant, it suggests a trend that may indicate that the mechanism controlling susceptibility in the Mcs $1 b$ locus is much more complex than originally thought. A resistant phenotype could be compensated for by the action of another risk allele lying outside of the Mcs $1 b$ locus on the distal side. Indeed, the GWAS by Easton et al. reported two additional alleles identified upstream of SNP 
$r s 889312$ and an additional breast cancer-associated allele was identified on human chromosome 5 in a subsequent breast cancer-GWAS (Easton et al., 2007; Turnbull et al., 2010). SNPs $r s 981782$ and $r s 30099$ reported by Easton et al. are positioned on human chromosome 5 at bases 45321475 and 52454339 , respectively. SNP rs981782 has a minor allele frequency of 0.47 in the SEARCH population studied and associates with a reduction in breast cancer incidence with an $\mathrm{OR}$ of $0.92(95 \% \mathrm{CI}=0.87-0.97)$ when homozygous for the minor allele (Easton et al., 2007). SNP rs30099 has a minor allele frequency of 0.08 in the SEARCH population and associates with an increase in breast cancer incidence with an OR of $1.09(95 \% \mathrm{Cl}=0.96-1.24)$ when homozygous for the minor allele (Easton et al., 2007). The magnitude of the affect associated with these loci is much smaller than what is seen for SNP $r s 889312$. Regardless, the results from the 1b11 phenotype suggest that an additional risk allele may be present in the $1 \mathrm{~b}-11$ locus. To address this, I used a bioinformatics approach to identify rat orthologs of human SNPs rs 981782 and $r s 30099$. I determined that LD blocks associated with these SNPs have orthologs on rat chromosome 2 (Figure 9A). SNP rs981782 lies in a 56kb haplotype block on human chromosome 5 and is in LD with one other SNP, $r s 4866929$ (Figure 9B). This haplotype block has a rat ortholog that spans from base pairs (bp) 49907723 to 49954324 on chromosome 2 (Figure 9A). SNP rs30099 is also in LD with only one other SNP, rs30727 and lies in a $3 \mathrm{~kb}$ haplotype block on human chromosome 2 (Figure 9C). The haplotype block has a rat ortholog that lies on chromosome 2 from bases 46916997 to 46918680 (Figure 9A). SNP rs 9790879 reported by Turnbull et al. lies within a 488 kb haplotype block on the $p$ arm of human chromosome 5 and is in LD with approximately 100 other SNPs (Figure 9D). There is a rat ortholog to this block 
A

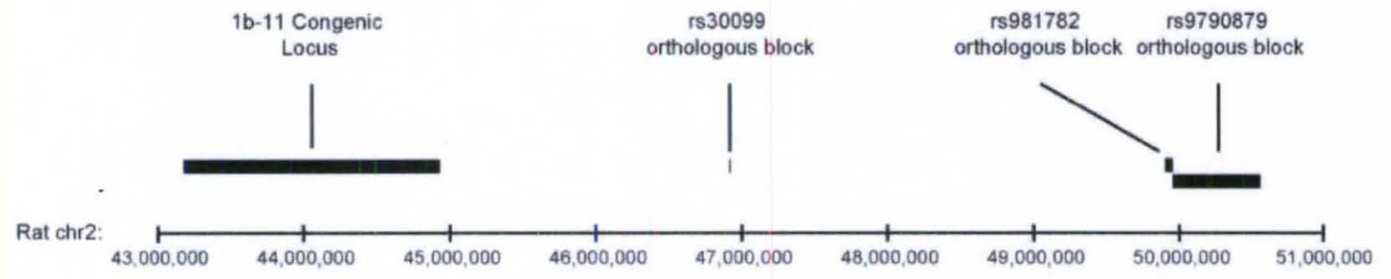

B

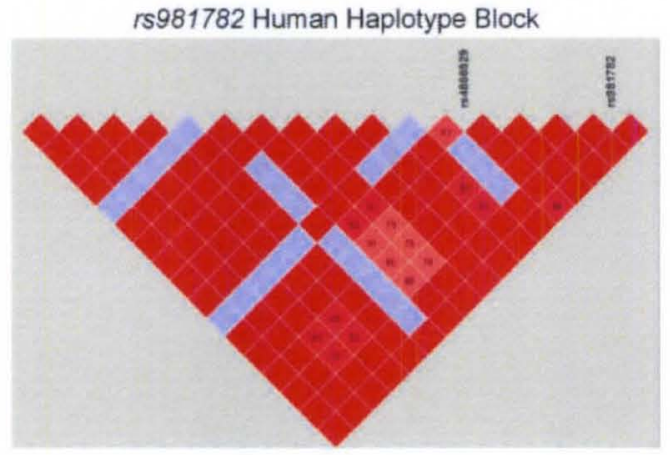

C

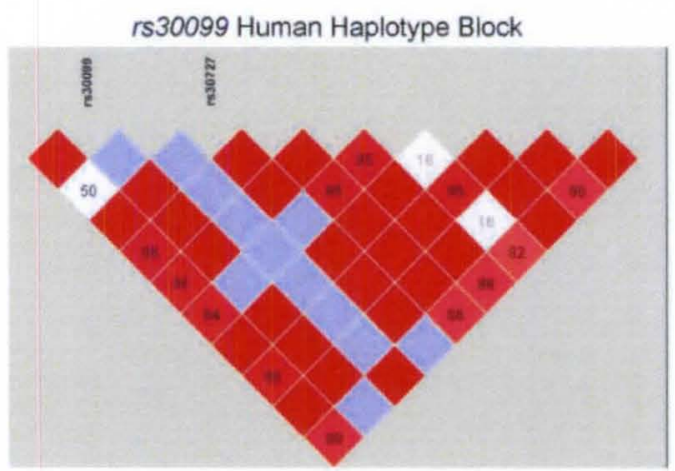

D

rs9790879 Human Haplotype Block

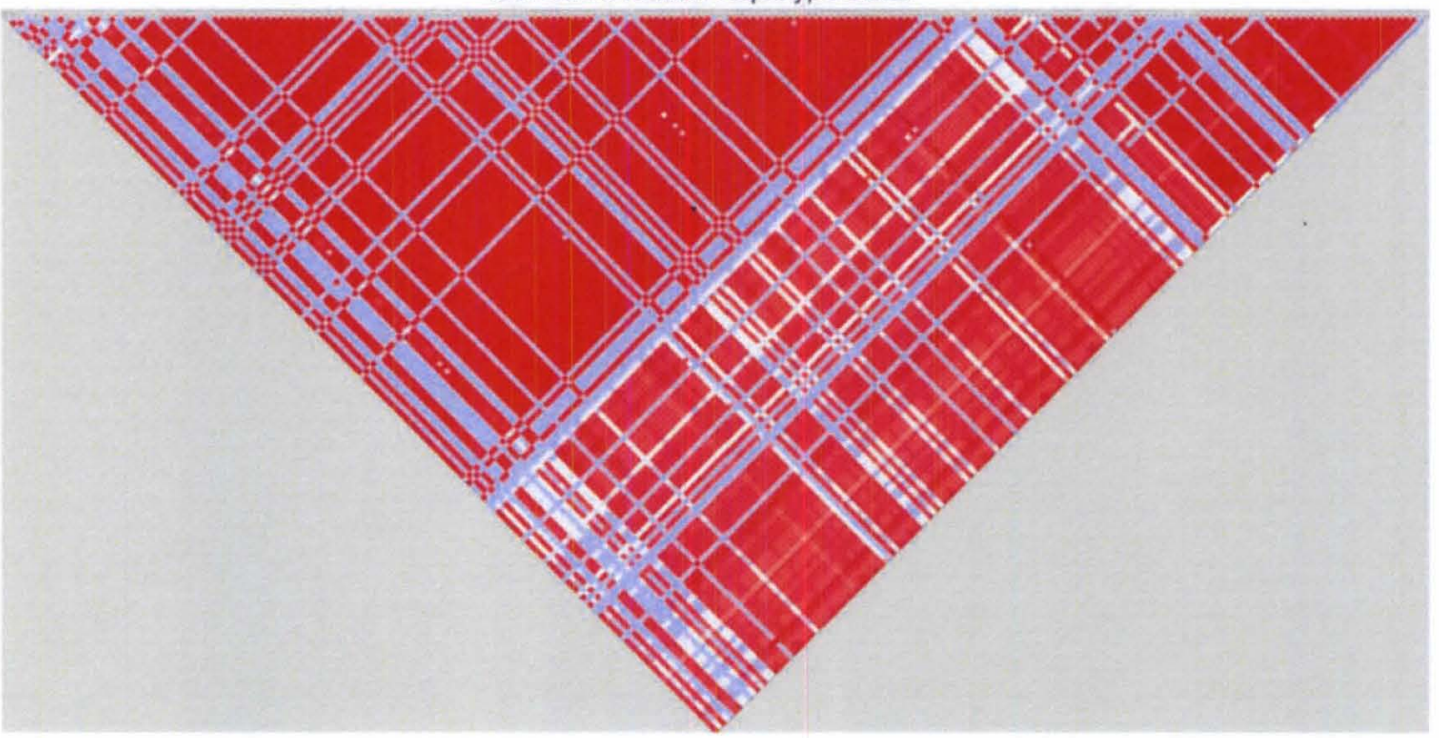


Figure 9: Additional breast cancer-associated loci identified in GWAS with rat chromosome 2 orthologs. A; Map of line 1b-11 interval on rat chromosome 2 showing positions of blocks orthologous to human haplotype blocks in LD with other breast cancer-associated SNPs. B - D; Haploview images of human haplotype blocks associated with individual breast cancer associated SNPs. Lower panel shows pairwise $\mathrm{r}^{2}$ values for LD; light blue represents lower disequilibrium, white is intermediate, and red represents strong disequilibrium. An $\mathrm{r}^{2}$ threshold of 0.80 was used to determine SNPs in LD. SNPS in LD are shown for $r s 981782$ and $r s 30099$. There are too many SNPs in LD with $r s 9790879$ to maintain resolution; therefore, these are not shown. 
delimited by bp 49961197 and 50564367 (Figure 9A). When mapped to the rat genome, none of these rat orthologs lie within the $1 \mathrm{~b}-11$ congenic region (Figure 9A). This negates the notion that there is a second allele that is offsetting the affect of the Mcs $I b$ locus. However, this is based on the assumption that there must be a human ortholog. It is possible that a second risk allele is present in this region but is specific to the rat or that an ortholog to a human risk allele lies within the $1 \mathrm{~b}-11$ locus yet unidentified in human studies. Testing this idea would require the development of new congenic lines containing varying portions of the distal end of line $1 \mathrm{~b}-11$ to define the specific region for this phenotype. However, this would be meaningless to human breast cancer susceptibility if the effect is rat-specific.

Another potential explanation for the susceptible phenotype seen in the $1 \mathrm{~b}-11$ line is that $M c s I b$ is a compound locus, i.e., resulting from the action of two or more variants working in concert to give rise to the phenotype. Line N3 shows a $\sim 56 \%$ reduction in tumor multiplicity compared to WF controls (Table 1). This indicates that the N3 allele contains the necessary variants required to give rise to the resistant phenotype. The $1 \mathrm{~b}-$ 11 interval overlaps the distal end of the N3 interval. Therefore, the $1 \mathrm{~b}-11$ allele could harbor a variant that is insufficient alone and requires an additional variant lying upstream. This variant would be present in N3 but lost in 1b-11, thereby eliminating the resistance phenotype in $1 \mathrm{~b}-11$ congenic rats. Work here is not conclusive, but other complex QTLs were identified that operate this way (Samuelson et al., 2007). To further study this potential mechanism would require modifying the current approach by incorporating cross-breeding of different congenic lines to determine whether the resistant phenotype can be restored, i.e., breeding of $\mathrm{U} 2$ or $\mathrm{W} 2$ rats to $1 \mathrm{~b}-11$ rats and 
phenotyping offspring containing both $\mathrm{U} 2 / \mathrm{W} 2$ and $1 \mathrm{~b}-11$ alleles. If $M c s l b$ is a complex locus, these offspring should exhibit a resistant phenotype.

Gould and colleagues show that line T heterozygous female rats average $7.6 \pm 0.8$ $(\mathrm{N}=18)$ mammary carcinomas per rat (Haag et al., 2003). This result was not statistically different from WF-homozygous littermates $(8.3 \pm 0.8, \mathrm{~N}=18)$ but was significantly increased $(\mathrm{P}<0.0001)$ compared to $\mathrm{COP}$-homozygous line $\mathrm{T}$ rats $(3.5 \pm 0.5, \mathrm{~N}=21)$. These data suggest that the WF allele at the line $\mathrm{T}$ locus exerts a dominant phenotype in a Mendelian sense. On the other hand, Line N3 heterozygous and COP-homozygous littermates develop $5.4 \pm 3.6(\mathrm{~N}=15)$ and $3.4 \pm 2.0(\mathrm{~N}=25)$ carcinomas per rat, respectively (Table 1). Both of these values are statistically reduced compared to WFhomozygous line $\mathrm{N} 3$ littermate female rats $(7.8 \pm 3.1, \mathrm{~N}=25)$. Tumor multiplicity in line N3 heterozygous rats is approximately half compared to COP-homozygous littermates signifying neither the WF nor the COP allele is acting dominantly. Taken together, these figures show that there is a decided difference between the line $\mathrm{T}$ and N3 alleles. This substantiates the notion that there is a more complex mechanism occurring at this locus. The simplest explanation is that there is an epistatic allele placed in either the proximal or distal regions flanking the $\mathrm{N} 3$ allele; however, other mechanisms may be at play. A compound locus could exist as described above. Moreover, it may be that there is a cisinteraction requiring a second element that is present in the line $\mathrm{T}$ interval but is absent in line N3. My data are insufficient to favor one scenario over another but suggest that the mechanism of action at this locus is much more complex than originally anticipated.

In addition to the $\mathrm{COP}$ rat, the $\mathrm{BN}$ rat strain is also almost completely resistant to developing spontaneous, chemically-induced, and oncogene-induced mammary 
carcinomas. As previously mentioned, the BN sequence has been completely sequenced. It is currently not known whether the resistant phenotype exhibited by the BN rat is associated with the Mcs $1 b$ locus. The BN rat was tested to determine if the mammary carcinoma resistant phenotype is controlled in part by the $M c s 1 b$ locus. If it is, the $\mathrm{BN}$ could be an additional tool to help discover SNPs that may be involved in the COP $M c s I b$ phenotype. A WF.BN $M c s I b$ congenic line had been initiated and I attempted to establish this line in order to determine if the $\mathrm{BN} M c s l b$ region confers resistance to developing mammary carcinomas in similar manner to the COP strain.

Results of WF.BN phenotype studies were to be applied to further characterize the Mcslb locus in the COP line as well as determine whether the Mcslb locus is involved in controlling mammary carcinoma susceptibility in the BN line. By examining the phenotype of the $\mathrm{BN}$ allele a 3-way haplotype analysis would rule in/out specific polymorphisms in the Mcs $1 b$ locus for their contribution to the mammary carcinoma resistance phenotype. The BN haplotype carries a set of SNPs that is shared with the $\mathrm{COP}$ allele ( $\mathrm{BN}=\mathrm{COP}$ at $A 12 v$ and $A 12 o o$ ) and can, therefore, be used to develop the $M c s 1 b$ locus with a BN haplotype. Markers between WF and COP are infrequent in the $M c s 1 b$ interval; however, the $\mathrm{BN}$ strain has been sequenced and many more markers have been identified. Therefore, these may be used to identify markers that may be used to narrow the Mcs $I b$ locus.

Unfortunately, however, the line was lost before the N10 generation and the $M c s I b$ region narrowed. Regardless, data from initial studies with the WF.BN congenic line at the N4F1 generation imply that the mammary carcinoma resistance exhibited by BN rats is, at least in part, controlled by the $M c s 1 b$ locus. This cannot be stated with 
complete confidence as the proximal end of the WF.BN rats contains a region of potential recombination that overlaps with the $M c s l c$ locus. Therefore, the $M c s l c$ locus could be involved. Additionally, these WF.BN congenics have large intervals of BN sequence that flank the distal side of the $M c s I b$ locus. These rats potentially harbor other risk alleles that are controlling mammary carcinoma susceptibility, i.e. the rat orthologs of the human haplotype blocks associated with SNPs $r s 981782$, rs30099 or rs 9790879 . With the loss of the WF.BN congenic line further investigation of the $\mathrm{BN}$ phenotype is not practical at this time.

Overall, the data delineate the $M c s 1 b$ locus to a $1.01 \mathrm{Mb}$ region flanked by SNP markers $A 12 v$ and D2Rat16. The variant(s) contributing to the Mcs $1 b$-conferred resistance phenotype likely lies inside this interval unless the mechanism is much more complex, as discussed. To determine the specific variant(s) contributing to mammary carcinoma susceptibility deep sequencing of this region is needed to identify new variants and test them accordingly. If no variant is identified lying inside the region delineated by $A 12 v$ and $D 2$ rat 16 , this strongly implicates that $M c s l b$ requires additional cooperative loci or that the 1b-11 line contains an epistatic allele downstream.

One of the goals for this project was to shorten the interval containing the Mcs $I b$ locus to minimize the list of potential gene candidates that would need to be functionally tested. This was achieved by mapping the $M c s l b$ locus to $1.01 \mathrm{Mb}$. Within the $1.01 \mathrm{Mb}$ Mcs $1 b$ locus there are five potential gene candidates: Actbl2, Gpbpl, Mier3, C5orf35 and Map3k1. Mier3, C5orf35 and Map3kI all lie inside the region orthologous to the rs 889312 LD block. Although these three transcripts are the most likely candidates, any one or combination of the genes contained within $M c s l b$ may be involved in controlling 
mammary carcinoma susceptibility and they must be tested further to determine their role. 


\section{CHAPTER III}

\section{RAT MCS1B ACTS IN A MAMMARY GLAND CELL-AUTONOMOUS MANNER TO CONTROL MAMMARY CARCINOMA SUSCEPTIBILITY}

\section{Introduction}

The etiology of mammary carcinogenesis is complex, involving events that occur within the mammary parenchyma as well as events that are external to the mammary gland. In both humans and rats, mammary tumors arise primarily in the cells that line the mammary duct, the ductal epithelial cells. One would expect, then, that genetic differences that give rise to sustained proliferation and carcinogenesis would originate in these cells. Likewise, it would be likely that anti-oncogenic properties, such as the phenotype conferred by the Mcs $1 b$ COP allele, also lie within these cells. Indeed, there are both oncogenes and tumor suppressor genes expressed in the ductal epithelia that affect tumor development; however, as mentioned previously, different groups have shown that other cell types external to the mammary gland can affect pathogenesis (Pollard, 2004; Sica et al., 2006; Smits et al., 2011b; Trimboli et al., 2009).

Consonant with data illustrating mammary tumors originating in the mammary epithelial cell (MEC) population, it has been shown previously that the effects of the Mcs1 COP locus are generally intrinsic to the mammary gland (Zhang et al., 1990). However, in these studies a slight mammary gland-external effect was observed, likely due to mixed effects of multiple QTLs: $M c s l a, b$ and $c$. It is necessary, then, to test the 
$M c s I b$ allele independently of the other COP Mcs alleles. This is critical to functionally analyze potential candidate genes. For instance, results of these mammary gland autonomy studies will guide us as to what tissues or cell types to examine when assessing transcript levels or protein expression. Moreover, knowing the site of function of the $M c s I b$ allele may lend some insight into a viable gene candidate or a plausible role that a gene may be performing to control mammary carcinoma susceptibility. There are five potential gene candidates lying in the narrowed Mcs Ib locus; Actbl2, Gpbp1, Mier3, C5orf35 and Map $3 k 1$. Of these, only Map $3 k 1$ has been fully characterized. Therefore, identifying the tissue or cell type that the Mcslb locus is conferring resistance to mammary carcinoma development may provide leads as to potential functions of these transcripts in the context of mammary carcinogenesis. Further, these mammary gland cell-autonomy studies are critical in that they provide the basis for all functional studies of the Mcslb locus and will potentially provide needed insight into the etiology of mammary carcinogenesis.

\section{Design and Methods}

\section{Whole Mammary Gland Transplant Assays}

Donor nulliparous female homozygous WF and line N3 WF.COP congenic rats ( $N=\sim 25$ animals per group) were euthanized at 30-35 days of age and their abdominal and inguinal mammary glands excised. The glands were scissor-minced and, using an IACUC-approved protocol, transplanted into the interscapular white fat pad of agematched nulliparous female rats in a reciprocal manner; i.e. WF into WF.COP and WF.COP into WF. Also, WF into WF and WF.COP into WF.COP transplants were 
performed as controls. At 50-55 days of age, recipient animals were administered DMBA dissolved in sesame oil $(20 \mathrm{mg} / \mathrm{ml})$ by oral gavage $(65 \mathrm{mg} / \mathrm{kg}$ body mass) to induce mammary carcinogenesis. At 15 weeks post-DMBA administration, recipient rats were necropsied and interscapular fat pads were examined for tumor development (Figure 10). Fat pads were whole mounted onto glass slides and stored in $70 \%$ ethanol for two weeks to allow for clearing of lipids from the pad. After two weeks, the whole mounts were fixed with 1 part glacial acetic acid and 3 parts $100 \%$ ethanol for 1 hour, hydrated by serially-decreasing ethanol washes $(70 \%, 50 \%, 40 \%$, distilled water) for 15 minutes each, and stained in aluminum carmine for four days. Aluminum carmine was prepared by dissolving $2.5 \mathrm{~g}$ alum potassium sulfate (Spectrum Chemical, New Brunswick, NJ) and $1 \mathrm{~g}$ Carmine stain (TCI America, Portland, OR) in $500 \mathrm{ml}$ distilled water, boiling for 20 minutes and filtering out precipitate. Once stained, whole mounts were dehydrated again with serially-increasing ethanol washes $(50 \%, 70 \%, 100 \%)$ and finally a xylene wash. Whole mounts were stored long term in mineral oil. To verify properly mammary gland development in the transplant site whole mounts were examined for proper mammary gland duct elongation and TEB formation. Whole mounted fat pads were examined microscopically for frank carcinoma, ductal carcinoma in situ (DCIS) and hyperplasia development. Tumor outcome was analyzed using logarithmic regression analysis. DCIS and hyperplasia multiplicity data were analyzed by Mann-Whitney rank test.

\section{Mammary Gland Cell Preparation and Injection into Interscapular Fatpad}




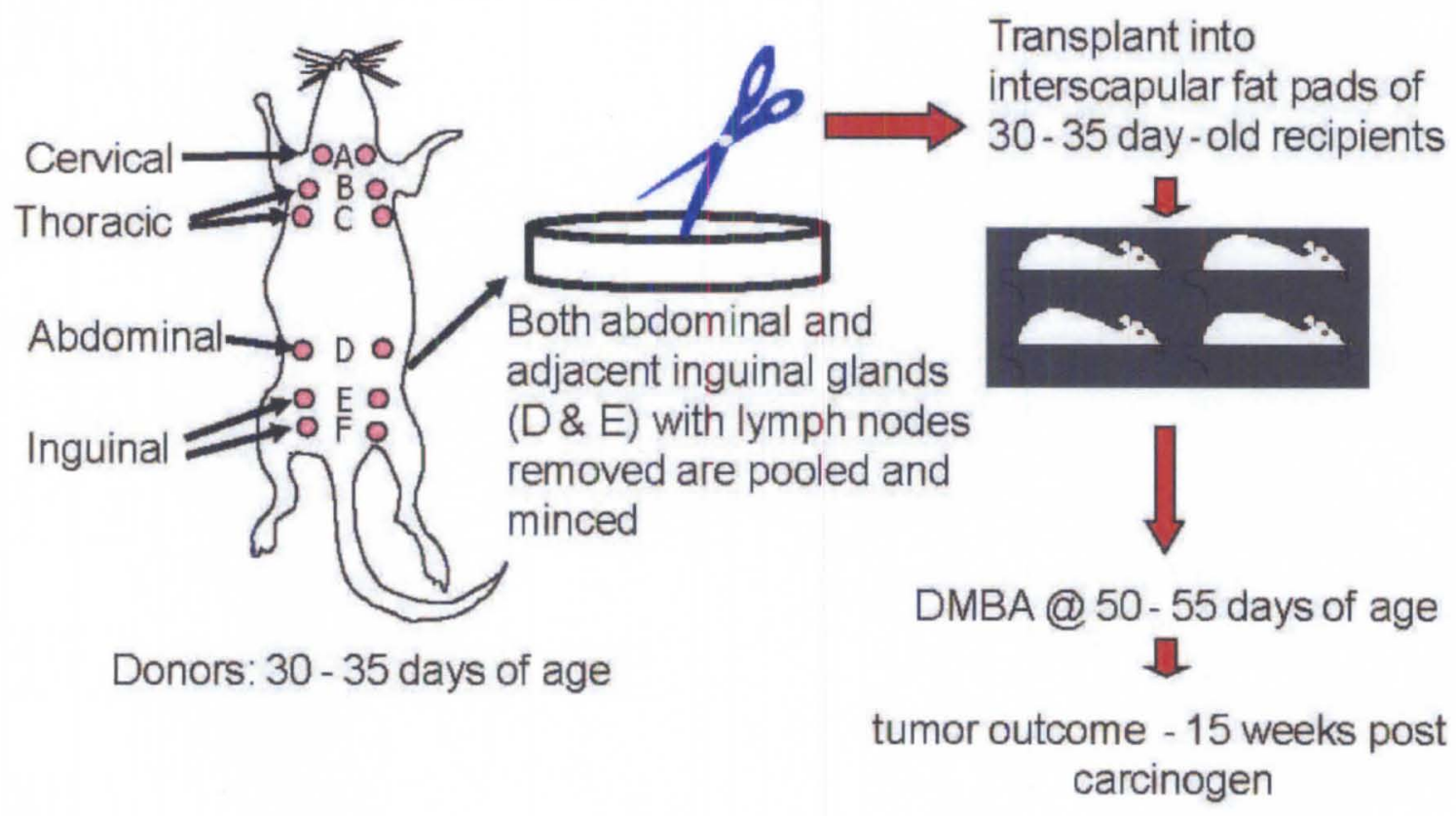

Figure 10: Flow diagram of mammary gland grafting assay. 
Donor nulliparous female homozygous WF and line N3 WF.COP congenic rats $(\mathrm{N}=\sim 25$ animals per group) were euthanized at 30-35 days of age and their abdominal and inguinal mammary glands excised. The glands were scissor minced and epithelial cells were enriched by enzymatic digestion in collagenase III (Worthington, Lakewood, $\mathrm{NJ}$ ) for 3-4 hours at $37^{\circ} \mathrm{C}$. Details of this method have been previously published (Gould and Clifton, 1985). The mammary epithelial cell-enriched suspensions (hereafter referred to as MEC preparations) were injected into the interscapular white fat pads of 30-35 day old nulliparious WF female recipients $\left(\mathrm{N}=16\right.$ animals per group) at $1 \times 10^{6}$ cells per animal in $100 \mu \mathrm{l}$ of DMEM/F12 (Life Technologies, ) media ( $1 \times 10^{7}$ cells $\left./ \mathrm{ml}\right)$. Whole mammary gland transplants from WF donors were performed as positive controls.

Tumor development in the ectopic mammary glands was examined 15 weeks following DMBA administration to determine the mammary carcinoma susceptibility of the ectopic mammary gland. Transplants that did not develop tumors were examined by whole mounting the fat pad and staining with aluminum carmine to confirm that the transplanted gland developed properly in the fat pad, as previously described. Fat pads that failed to develop mammary glands greater than $3 \times 3 \mathrm{~mm}^{2}$ were excluded from further analysis. Previous studies utilizing this method have reported that a very small percentage of recipient rats develop multiple tumors in the transplant site. Therefore, tumor outcome was assessed as a binary response and analyzed by logistic regression.

\section{Mammary Gland Cell Injections supplemented with Adherent Cell Fraction}

Mammary gland cell preparations were made from WF donors using the mammary gland cell preparation protocol described above. Adherent cells left in the 
flask were removed with Trypsin +EDTA (Life Technologies) for 5 minutes and placed into $15 \mathrm{ml}$ centrifuge tubes. Approximately $250 \mu \mathrm{FBS}$ was gently added to the bottom of each tube to form a distinct layer for the cells to be centrifuged into. The cell solution was centrifuged briefly to collect cells and the supernatant discarded. The cell pellet was resuspended in media and counted and herein referred to as adherent cell fraction (ACF) preparations. ACF preparations were added to MEC preparations at 1:10 ratio resulting in a final MEC concentration of $1 \times 10^{7}$ cells $/ \mathrm{ml}$ plus a final ACF concentration of $1 \times 10^{6}$ cells $/ \mathrm{ml}$. MEC/ACF preparations were injected into the interscapular fat pads of nulliparious WF female rats. MEC preps alone were injected into the fat pads of nulliparous WF female rats as a control. At 12 weeks of age all recipient rats were administered DMBA. Recipient rats were necropsied 15 weeks post-DMBA and examined for tumor development in the ectopic site. Fat pads were mounted and processed and stained with aluminum carmine. Stained fat pads were evaluated for proper mammary gland development and hyperplasia and DCIS development was assessed. Tumor, hyperplasia and DCIS data were analyzed using a Mann-Whitney rank test.

\section{$\underline{\text { Results }}$}

\section{Whole Mammary Gland Transplants Suggest Rat Mcs1b Is Mammary Cell}

\section{Autonomous}

To determine if the $M c s 1 b$ COP allele reduces mammary carcinoma susceptibility in a manner that is intrinsic to the mammary gland, rats were transplanted with whole mammary gland tissue into their interscapular fat pads and exposed to DMBA (Figure 
10). All of the donor and recipient female rats used had a WF background and only differed at the Mcs $I b$ locus having either a resistant COP or susceptible WF allele. We expected that there would be no rejection of the graft as both genotypes have the same major histocompatibility complex (MHC) type and, thus, have compatible immune systems. To verify this, we determined that recipients did not reject mammary tissue grafts from donors of different genotypes by microscopic assessment of whole mounted fat pads. There was no statistically significant association $(\mathrm{P}=0.1869)$ between the outcome of ectopic mammary gland development and donor or recipient genotype (Table $6)$.

Data from ectopic mammary gland-positive fat pad whole mounts were analyzed for associations of donor and recipient genotypes with ectopic carcinoma development. Ectopic mammary glands formed from mammary gland tissue from $M c s l b$ resistant donors, when grafted into either resistant or susceptible recipients, resulted in fewer ectopic tumors forming compared to ectopic mammary glands from susceptible donors when grafted into animals of either genotype (Figure 11). The genotype of the donor was significantly associated $(\mathrm{P}=0.0019)$ with ectopic mammary carcinoma outcome while there was no effect seen from the recipient genotype (Table 7). These data signify that the resistant phenotype conferred by the $M c s 1 b$ COP allele is acting in a mammary glandautonomous manner. Based on this, future studies to functionally characterize the $M c s 1 b$ locus should be focused on the mammary gland.

Enzymatically-Isolated Mammary Gland Cells Injected into the Interscapular Fat Pad Reconstitute a Normal Mammary Gland in the Ectopic Site 
Table 6:

Graft site MG development outcome (dependent) and Mcs $1 b$ donor and recipient genotypes (independent)

\begin{tabular}{lccc}
\hline & Coefficient & P value & $\begin{array}{c}\text { Odds Ratio } \\
\text { (95\% CI) }\end{array}$ \\
\hline Donor Effect & 0.99 & 0.1869 & $\begin{array}{c}2.69 \\
(0.62-11.68) \\
0.27 \\
\text { Recipient Effect }\end{array}$ \\
Intercept & -1.31 & 0.116 & $(0.05-1.38)$ \\
\hline
\end{tabular}




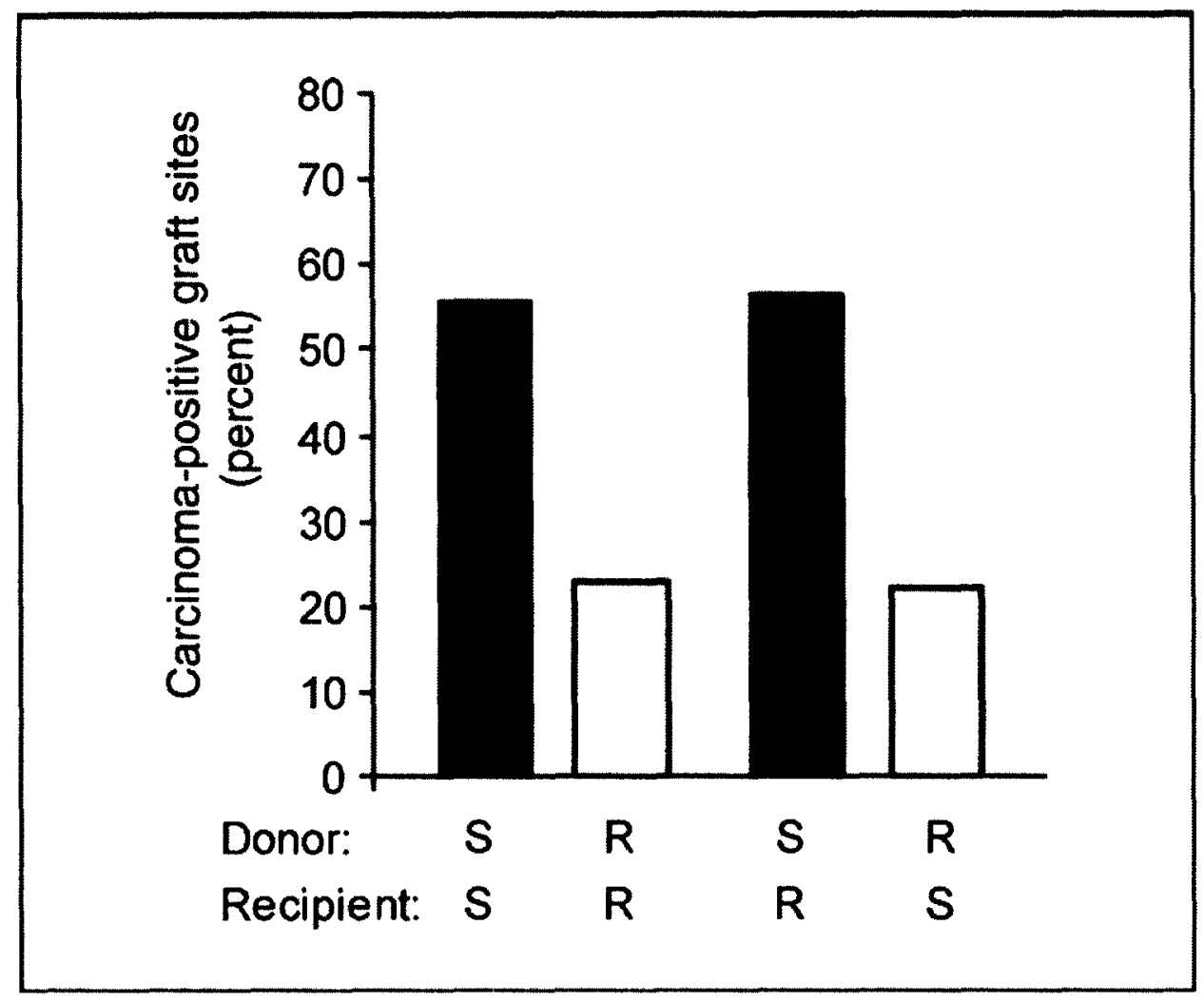

Figure 11: Rat $M c s 1 b$ is mammary gland autonomous. Percentage of mammary glandgraft-positive recipients that developed ectopic mammary gland carcinomas are shown for each susceptible (S) and $M c s 1 b$-resistant (R) donor:recipient group. Groups with $\mathrm{S}$ donors are shown as filled bars, and groups with $\mathrm{R}$ donors are shown as unfilled bars. The total number of mammary gland-graft-positive recipients that were evaluated for tumor outcome in each group were, respectively, 27, 22, 23, and 18 for $S: S, R: R, S: R$, and $\mathrm{R}: \mathrm{S}$. 
Table 7:

$\underline{\text { MG graft site tumor outcome (dependent) and } M c s 1 b \text { donor and recipient genotypes }}$ (independent)

\begin{tabular}{lccc}
\hline & Coefficient & P value & $\begin{array}{c}\text { Odds Ratio } \\
\text { (95\% CI) }\end{array}$ \\
\hline Donor Effect & 1.48 & $0.0019^{*}$ & $\begin{array}{c}4.4 \\
(1.73-11.18) \\
0.96 \\
\text { Recipient Effect }\end{array}$ \\
Intercept & -0.04 & 0.9381 & $(0.39-2.36)$ \\
\hline
\end{tabular}


The $M c s 1 b$ COP resistance effect is intrinsic to a mammary gland cell type. As mentioned, the majority of rat mammary tumors arise in the epithelial cells lining the duct. In addition to these ductal epithelial cells there are myoepithelial cells that surround the duct and TEB and multipotent cap cells that can differentiate into myoepithelial cells or move inward and differentiate into luminal cells (Williams and Daniel, 1983). Based on the fact that mammary tumors originate in the cells lining the mammary gland duct we hypothesized that one or more of these epithelial cell types are responsible for Mcs $1 b$ COP-conferred resistance. However, there is evidence that other cell types in the mammary mesenchyme affect tumor development (Pollard, 2004; Sica et al., 2006; Smits et al., 2011b). To test which cell type the Mcs $1 b$ COP allele is acting on to control mammary carcinoma development I used a MEC-enriched clonogen transplant approach that had been previously developed (Gould and Clifton, 1985). This method is used to enrich for MECs and non-adherent leukocytes and lymphocytes by removing mammary gland adipocytes, fibroblasts, and other strongly adherent cells by negative selection. These cells are injected into the interscapular fat pad of recipients to induce development of an ectopic mammary gland similar to the whole mammary gland transplantation experiments.

Enzymatic preparation of mammary glands to enrich for MECs is the standard protocol. However, this method is time-consuming and I wished to decrease the time required to dissociate mammary glands. To do this, I compared cells dissociated mechanically using a Medicon tissue dissociator to cells from enzymatic preparations to determine whether they recapitulated a morphologically normal mammary gland in the transplant site. Rats receiving mechanically-dissociated MECs did not develop 
mammary glands in the ectopic site while $75 \%$ of the rats injected with enzymaticallyisolated MECs developed ectopic mammary glands when a 1:1 donor:recipient ratio was used (Table 8). Mammary glands grown in the ectopic site looked morphologically normal (Figure 12). Based on these results, subsequent MEC preparations were performed using the enzymatic method.

To estimate the number of rats to use for these studies we used standard deviations (SD) data from previous mammary cell autonomy experiments resulting in SD of approximately 0.6 ectopic mammary tumors. For a $95 \%$ confidence interval and using a tolerable error of \pm 0.15 of the mean ectopic mammary tumors with a SD of 0.6 , approximately 60 animals must be tested. Given we achieved $75 \%$ transplant efficiency in the pilot study, to be successful 80 animals per group are required.

\section{Mammary Gland Cell Preparations Are Not Sufficient to Allow for Efficient Mammary Carcinoma Development at Ectopic Graft Sites}

Since injection of MECs into the interscapular fat pad resulted in mammary glands formed in the ectopic transplant site, I intended to use this method to determine if the Mcs $1 b$ locus was controlling mammary carcinoma susceptibility in MECs. MECs isolated from susceptible WF $(\mathrm{N}=16)$ or resistant WF.COP $(\mathrm{N}=14)$ rats and injected into the fat pads of age-matched susceptible WF female rats to determine the effect of the genotype on tumor outcome in the transplant site. Upon inspection of stained whole mounts, all recipients $(100 \%)$ exhibited mammary gland development in the ectopic site. However, tumor development was not significantly different $(\mathrm{P}=0.9221)$, as each group developed only one tumor each (Figure 13A). Tumor outcome in MEC-derived 
Table 8:

Mammary gland outcome in enzymatically-dissociated versus mechanically-dissociated mammary gland cell preparations.

\begin{tabular}{lccc}
\hline $\begin{array}{c}\text { MEC isolation } \\
\text { method }\end{array}$ & $\begin{array}{c}\text { \# Rats } \\
\text { Administered }\end{array}$ & $\begin{array}{c}\text { \# Ectopic Mammary Glands } \\
\text { formed }\end{array}$ & $\begin{array}{c}\% \\
\text { Positive }\end{array}$ \\
\hline Medicon & 3 & 0 & 0 \\
Collagenase Digestion & 4 & 3 & 75 \\
\hline
\end{tabular}




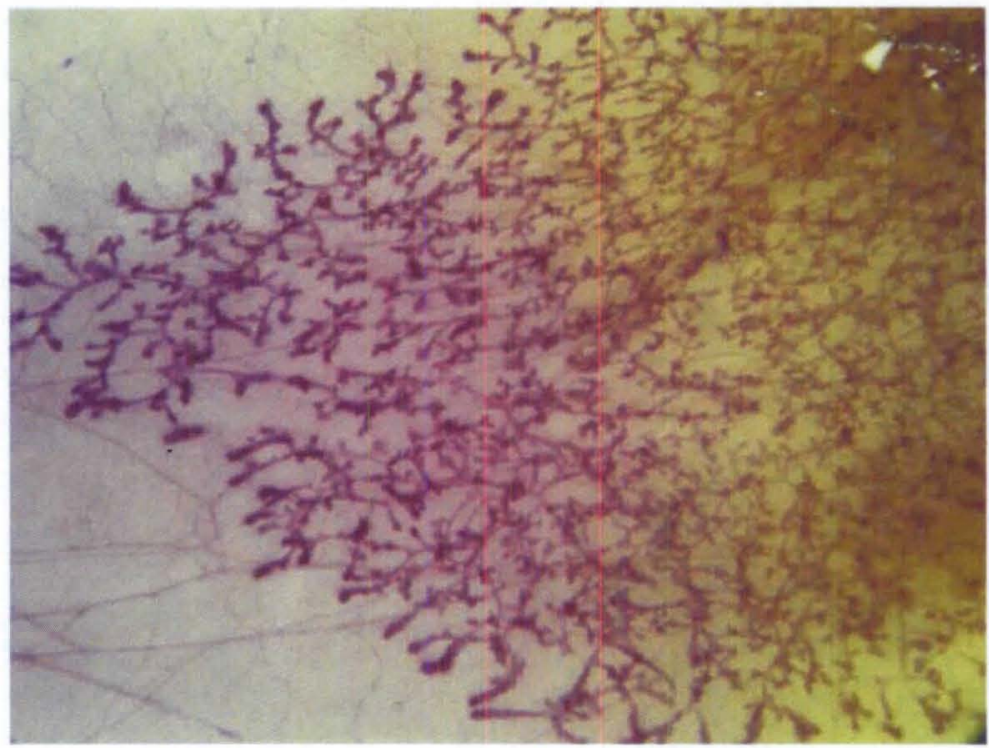

Figure 12: Representative whole mounted interscapular fat pad from MEC pilot study. 10X magnified image of aluminum carmine stained mammary gland formed in the fat pad following injection of MECs isolated via collagenase digestion. 
mammary glands was determined to be significantly reduced when analyzed against whole mammary gland transplant controls $(\mathrm{P}=0.0007$, Figure $13 \mathrm{~A})$.

Since tumor development was considerably reduced in animals receiving mammary gland cell preparations compared to whole mammary gland transplants, I examined the fat pads microscopically to determine if there were any gross morphological changes compared to ectopic mammary glands developed from whole mammary gland transplants. There did not appear to be gross morphological differences in ductal branching or TEB formation at the microscopic level; however, hyperplasia and DCIS formation was detected in the majority of whole mounts (Figure 12B). When quantified, hyperplasia were not significantly different $(\mathrm{P}=0.4884$, Kruskal-Wallis test $)$ between ectopic mammary glands from recipients of WF, WF.COP, or whole mammary gland transplants (Figure 12C). DCIS development was not significantly different $(\mathrm{P}=0.3545$, Mann-Whitney test) between ectopic mammary glands formed from MECs from either genotype (Figure 13C). However, DCIS formation in whole mammary gland transplants was significantly greater ( $\mathrm{P}=0.0003$, Mann-Whitney test) than for ectopic mammary glands formed from MECs of both genotypes.

\section{Injection of Mammary Gland Cell Preparations Along With Adherent Cell Fractions}

\section{Does Not Allow for Mammary Carcinoma Development at Ectopic Graft Sites}

Since there was no distinction in tumor outcome between ectopic mammary glands from susceptible and resistant MEC preparations and the ability to form frank carcinomas was nearly ablated, I sought to determine what was missing in cell preparations that was necessary for tumorigenesis. The MEC isolation method I 

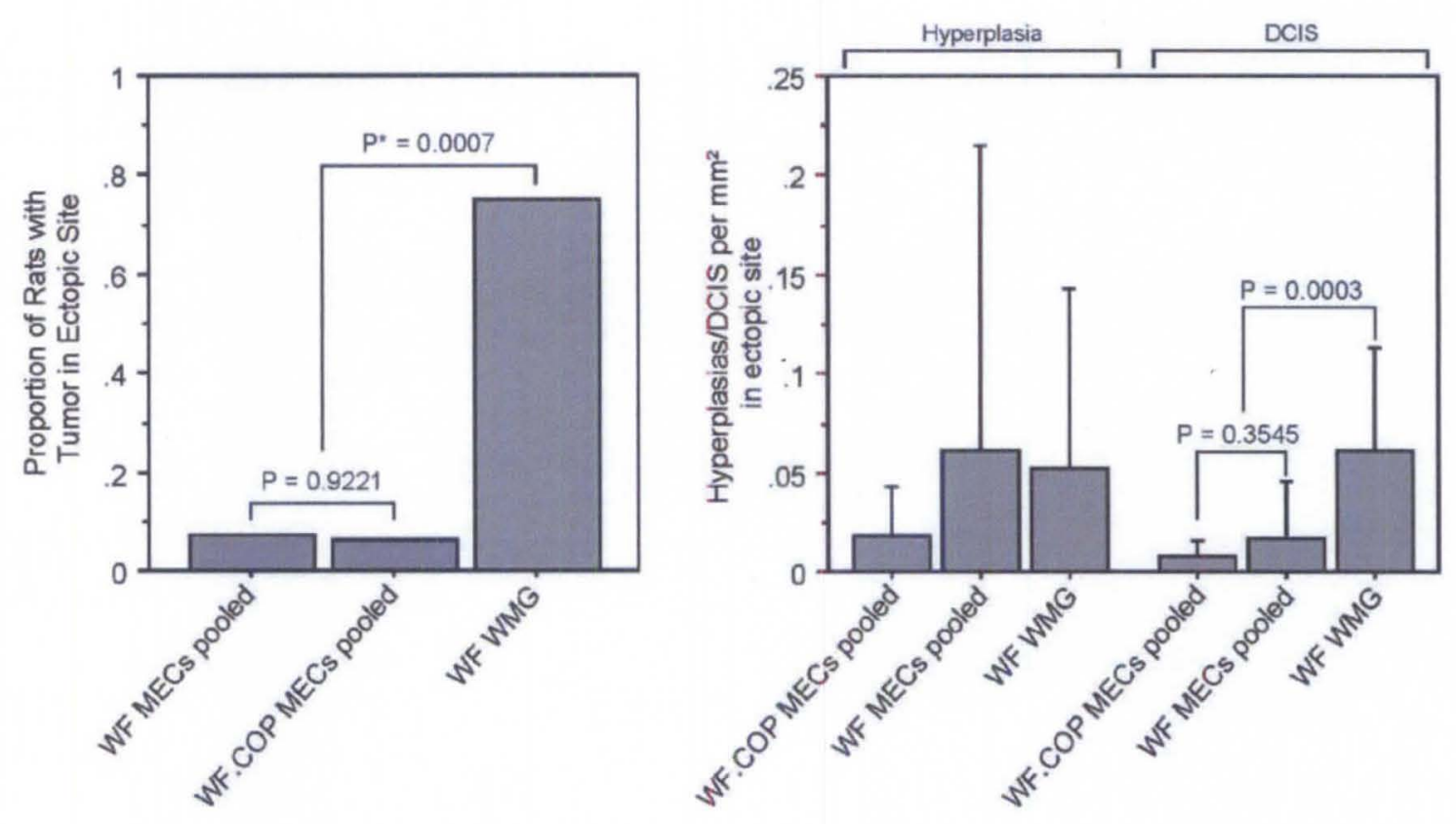

B

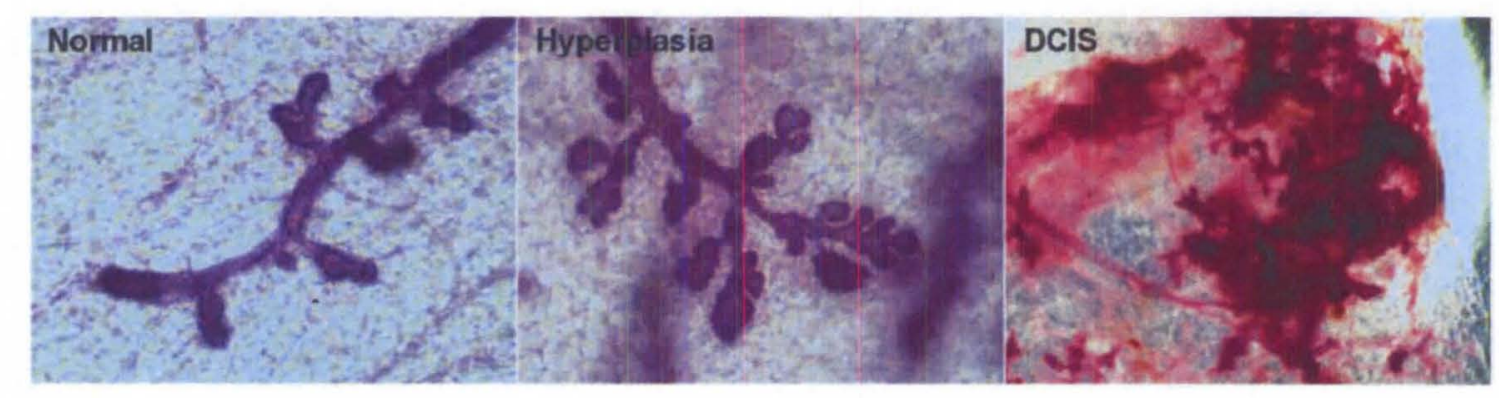

Figure 13: Tumor outcome in ectopic mammary glands derived from MECs. A; Proportion of interscapular fat pads with ectopic mammary glands exhibiting tumor development. P values based on logistic regression analysis. B; Microscopic images of aluminum carmine-stained normal TEB, hyperplastic TEB, and precancerous lesion stained from interscapular fat pad whole mounts. C; Quantification of hyperplasia and DCIS formed per $\mathrm{mm}^{2}$ in ectopic mammary glands. P values based on nonparametric Mann-Whitney test. 
originally employed enriches MECs by negative selection removing most adherent cells, i.e., fibroblasts, macrophages, mast cells, as well as, adipocytes, which remain on the top layer when centrifuged. These cell types play roles in mammary gland function; therefore, we hypothesized that the absence of one or more of these cell types resulted in retarding tumor development. To test this I adapted the original MEC isolation method to include the ACF that was discarded in the original protocol. These ACFs were retained and injected along with MECs. MEC-only injections and whole mammary gland transplants were performed as controls. I was attempting to restore the DMBA-induced mammary tumorigenesis susceptibility phenotype seen previously with WF whole mammary gland transplants; therefore, resistant cells were not considered and only WF donor cells were used.

I anticipated that co-injecting the adherent cells lost during dissociation along with MECs would restore mammary tumor development in the transplant site. Surprisingly, there was no difference $(\mathrm{P}=0.6664)$ in ectopic tumor outcome in WF rats receiving WF MECs $(\mathrm{N}=9)$ and WF MECs co-injected with the AHC $(\mathrm{N}=9)$ but, combined, these were significantly different from those receiving whole mammary gland transplants demonstrating that inclusion of the ACF was not sufficient to restore tumorigenesis (Figure 14A). Additionally, I quantified hyperplasia and DCIS formation microscopically by counting hyperplastic TEBs or DCIS foci. I noted no significant difference $(\mathrm{P}=0.8345$, Kruskal-Wallis test $)$ in hyperplasia formation between ectopic glands formed from MECs, MEC plus ACF cells or whole mammary gland transplants. DCIS development did not statistically differ between rats receiving WF MECs and those getting WF MECs plus the adherent cell fraction. However, DCIS formation was 

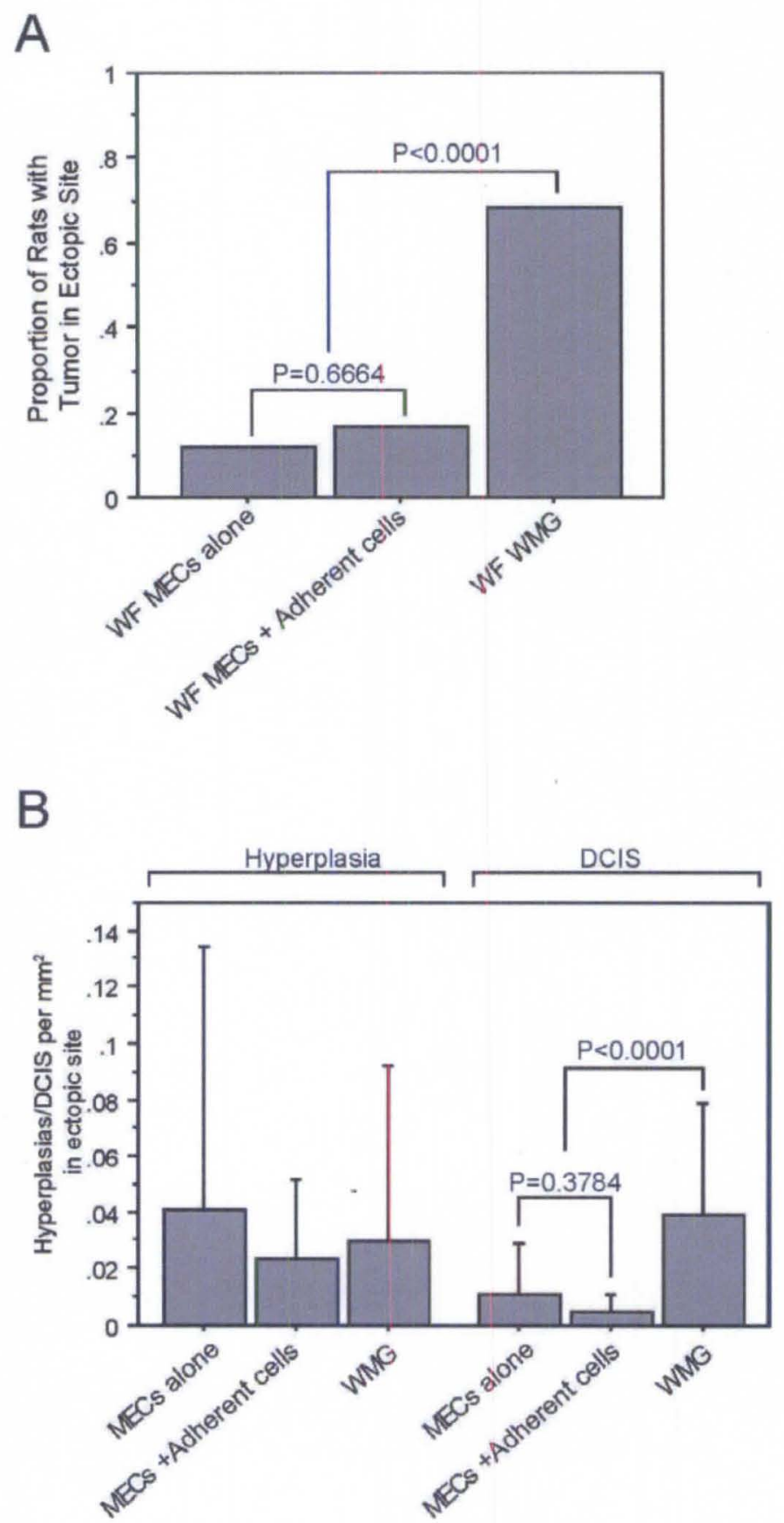

Figure 14: Tumor outcome in ectopic mammary glands derived from MECs or MECs supplemented with adherent cells. A; Proportion of interscapular fat pads with ectopic mammary glands exhibiting tumor development. P values based on logistic regression analysis. B; Quantification of hyperplasia and DCIS formed per $\mathrm{mm}^{2}$ in ectopic mammary glands. P values based on nonparametric Mann-Whitney test. 
significantly increased $(\mathrm{P}<0.0001)$ in whole mammary gland transplants compared to either cell injection (Figure 14B).

DMBA Susceptibility Is Delayed In Ectopic Mammary Glands Formed from Injection of Mammary Gland Cell Preparations

Injection of cellular components of mammary glands into the interscapular white fat pad failed to form tumors in the resulting ectopically-formed mammary gland. Coinjecting the AHC along with MECs again showed no difference in formation of frank carcinomas, DCIS or hyperplasia suggesting that there was a more complex process taking place. Within the process of dissociation of the mammary gland for MEC enrichment adipocytes are removed. Adipocytes are critical in mammary gland development and could be the missing link in efficient tumor formation that is lost in the mammary cell preparation transplants. However, there is an adequate concentration of host adipocytes within the interscapular fat pad cellular milieu making this less likely. Additionally, extracellular matrix (ECM) proteins are removed during MEC enrichment by collagenase digestion. The ECM provides an essential substrate required for normal mammary gland development; therefore, ECM may be required for tumor growth in these ectopic mammary glands. However, reintegrating MEC preparations with the adherent cells would restore fibroblasts which are responsible for ECM deposition, making this scenario less likely as well. DMBA-induced mammary carcinogenesis in the rat has been established to be dependent on a distinct mammary gland developmental window of 5055 days of age; however, this window is based on fully developed mammary glands (Russo and Russo, 1994). We hypothesized that a different time span is required for 
dissociated mammary gland cells to reconstitute a functional mammary gland than for whole gland transplants, i.e., the DMBA-susceptibility window is shifted.

To test this idea I used the original MEC isolation protocol, including susceptible and resistant MEC preparations only and administered DMBA at $40-45,50-55,60-65$, and 70-75 days of age days. By including susceptible and resistant donor MECs, I would be able to identify whether the Mcs $1 b$ COP resistant phenotype is acting in the MEC population.

Interestingly, only $32 \%$ ( 8 of 25 ) of female rats receiving DMBA at the $40-45$ day susceptibility window developed a mammary gland in the interscapular fat pad while those receiving DMBA at $50-55,60-65$, or $70-75$ days of age developed $81 \%, 74 \%$, and $68 \%$, respectively (Figure $15 \mathrm{~A}$ ). This may suggest that administering DMBA too early after MEC injection may affect the efficiency of the graft to develop into a proper mammary gland.

Tumor outcome in the ectopic site was higher at the 40-45, 60-65, and 70-75 day susceptibility windows compared to the 50-55 day window (Figure 15B). These results are without regard to genotype of the MEC transplant as no genotype effect was detected (Figure 15B, bottom). Results for the $40-45$ and $60-65$ day windows were not statistically different from that of the original 50-55 day window $(\mathrm{P}=0.2352$ and 0.0659 , respectively). However, fat pad whole mounts from rats that received DMBA at 70-75 days exhibited a significantly higher tumor outcome when compared to the original 50-55 day DMBA window $(\mathrm{P}=0.0041)$. Furthermore, tumor outcome for the $70-75$ day window was not significantly different than what was observed in whole mammary gland transplants $(\mathrm{P}=0.2025)$. These results suggest a shift in the susceptibility window of 
A

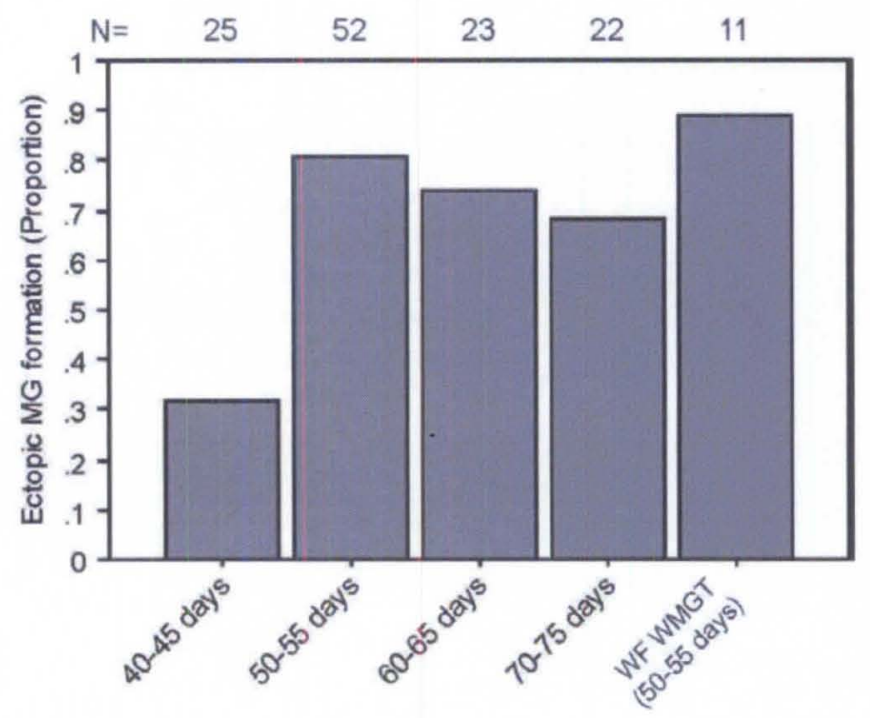

B

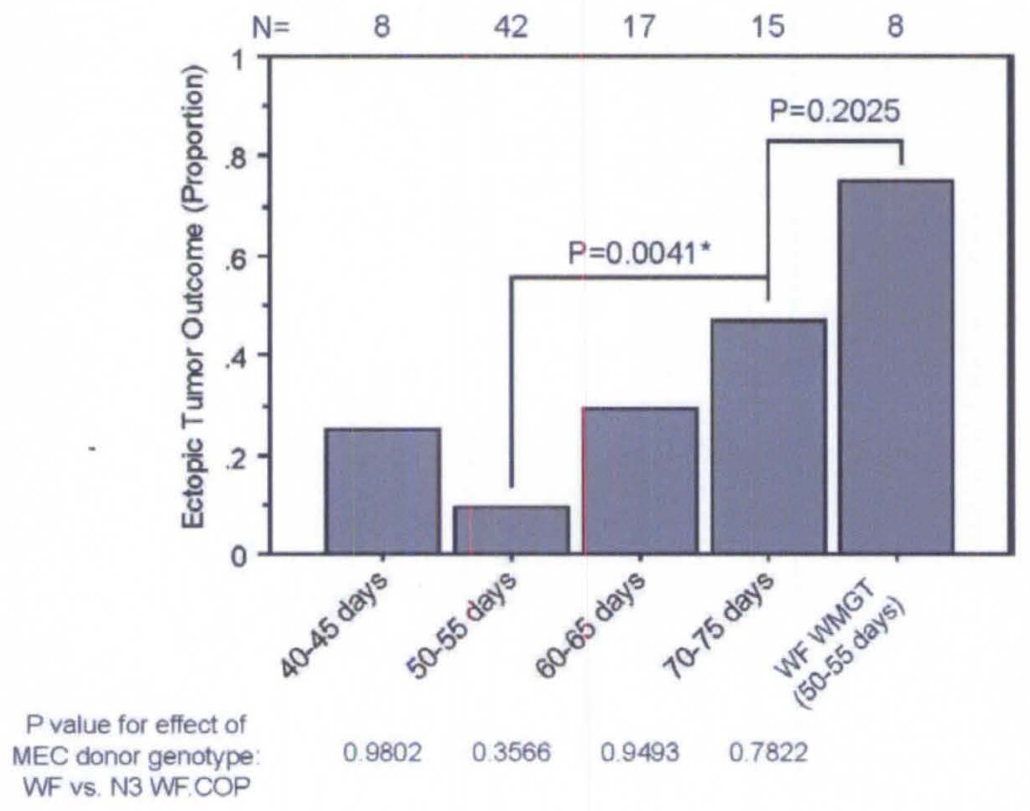

Figure 15: Results of DMBA susceptibility window studies with MEC injections. A; Mammary gland development outcome in the interscapular fat pad. Bars represent the proportion of rats injected that developed a mammary gland in the injection site. B; Tumor outcome in the ectopic mammary gland at varying windows of DMBAadministration. P values are based on logistic regression analysis. 
ectopic mammary glands derived from MECs compared to those from whole mammary glands. Also, since no genotype effect was observed it is likely that the action of the $M c s I b$ locus to control mammary carcinogenesis is not intrinsic to a mammary epithelial cell type.

\section{$\underline{\text { Discussion }}$}

Carcinogenesis in the mammary gland is complex involving multiple cell types and signaling mechanisms. On these lines, we set out to determine if the resistance to mammary carcinoma development conferred by the $M c s 1 b$ locus was acting in a mammary gland-intrinsic or extrinsic manner. The majority of mammary carcinomas arising in the rat originate in the ductal epithelium; thus, we hypothesized that the $M c s I b$ locus was acting within a mammary gland cell type to confer resistance.

Transplanting immature mammary glands into the interscapular fat pad results in the development of a properly branched and formed mammary gland (Gould and Clifton, 1985). We used this approach and induced mammary carcinogenesis with a single dose of DMBA. The results of these experiments supported our hypothesis that the Mcs $1 b$ conferred phenotype is mammary gland intrinsic.

Transcripts within the Mcslb locus remain to be functionally tested and these results indicate that the mammary gland should be the focus of these assays, e.g., gene expression profiles and splice variant analysis. Moreover, these data focus on a cell type of interest. Cells from the immune system can have an effect on mammary carcinogenesis in the tumor microenvironment (Pollard, 2004; Sica et al., 2006; Smits et al., 2011b; Trimboli et al., 2009). Herein, we have shown that this is not likely the case 
and focused on the mammary gland cellular milieu. The mammary gland is composed of a variety of cell types all of which have roles in normal gland development as well as progression to a neoplastic and malignant state (Lanigan et al., 2007). Therefore, identifying a candidate cell type will afford a greater understanding of the etiology of mammary cancer.

Given that rat mammary tumors arise predominantly in the epithelial cells lining the ducts, I hypothesized that MECs were responsible for the $M c s l b$-conferred phenotype. To test this I modified the original transplant protocol by injecting MECenriched cell preparations and tracking the phenotype. I anticipated that approximately twice as many WF MEC-derived ectopic mammary glands would exhibit tumor development than glands developed from WF.COP MEC donors, similar to what was seen in whole mammary gland transplant assays. However, the ability to form tumors in the transplant site was lost, suggesting that there is a component missing necessary for tumor development.

I estimated that 80 animals of each genotype would have to be tested to estimate a mean tumor outcome to within \pm 0.15 ; however, I only tested 16 homozygous WF and 14 resistant congenic WF.COP rats in these studies. Although this is below the target number, the ability to form tumors in the fat pad was nearly completely ablated in all animals. Moreover, the lack of tumor development was not an artifact of poor transplant efficiency since the majority of recipients $(97 \%)$ developed mammary glands in the interscapular fat pad. These ectopic glands appeared to have developed normally and, upon microscopic inspection, exhibited hyperplasia and DCIS development suggesting that DMBA was sufficiently initiating carcinogenesis within the ectopic site. Hyperplasia 
and DCIS were quantified to determine if there was a difference between the MEC and the whole mammary gland transplants. No significant difference in hyperplasia was identified between ectopic mammary glands formed by MECs from the two genotypes, nor were these different than hyperplasia in whole mammary gland transplants. Similarly, DCIS formation was not statistically different in ectopic glands formed from congenic WF.COP MECs compared to ectopic glands from homozygous WF MECs. However, DCIS formation in ectopic mammary glands from either genotype was much lower than what was observed in whole mammary gland transplants suggesting carcinogenesis was being initiated in the MEC-derived glands but something involved in the MEC-isolation process removed a component required for progression to a frank carcinoma.

My hypothesis was that a cellular component was removed during MEC-isolation that was required for complete tumor-induction by DMBA. To address this hypothesis, I first attempted to restore the ability to form tumors by reclaiming the cells removed during MEC enrichment and re-introducing them along with MECs into the fat pad and following the phenotype. However, including these adherent cells with MECs had no effect on tumor outcome compared to MECs alone, suggesting that the mechanism is more complex. The loss of tumorigenisis could be explained by the absence of adipocytes since these were discarded during MEC-enrichment. However, this seemed unlikely since the recipient fat pad contains adequate adipocyte content and allowed for mammary gland development in the majority of recipients. Therefore, it appeared that this effect could not be explained simply by a single missing cellular component.

Whole WF mammary gland transplants were performed as controls in these 
experiments and these exhibited a normal ectopic tumor outcome. Also, hyperplasia and DCIS development was quantified in ectopic glands from MEC-, MEC plus adherent cell-, and whole mammary gland-induced grafts. All grafts exhibited hyperplasia development, but there was no statistical effect associated with MECs, MECs plus adherent cells, or whole mammary gland transplants. Moreover, DCIS formation was not statistically different between ectopic mammary glands formed from MEC or MEC plus adherent cell grafts; however, again the level of DCIS formation in either cell-derived ectopic gland was significantly reduced compared to whole mammary gland grafts.

Taken together, these data suggest that DMBA is driving neoplastic events to form hyperplasia and precancerous DCIS lesions but is insufficient for progression to development of frank carcinomas. We hypothesized that there was a developmental gap in these ectopic mammary glands affecting their susceptibility to DMBA-induced carcinogenesis. I sought to determine if there was a difference in susceptibility to DMBA in WF MEC-grafted ectopic mammary glands at different developmental windows. DMBA was administered in previous experiments at 50-55 days of age of the recipient rat; approximately 20 days after cell injections were performed. Rather than adjust the age at which cell injections were performed, we chose to keep this constant at 30-35 days and, instead, administer DMBA at 4 different time points: 40-45 days, 50-55 days, 60-65 days, and 70-75 days. If there was an effect from the developmental stage of the ectopic mammary gland, it was anticipated that one of these windows would return the mammary carcinogenesis phenotype towards a level similar to that seen in the whole mammary gland transplant studies. One may predict that an earlier DMBA window would likely result in an increase in tumor formation as these cells would be expected to be more 
mitotic and, therefore, more vulnerable to DNA damaging agents. However, outcomes of the susceptibility window tests showed that female rats receiving DMBA at the 70-75 day window developed significantly more tumors than rats receiving DMBA at the $40-45,50$ 55 , and 60-65 day windows. Tumor outcome in the 70-75 day group was not statistically different than that observed in whole mammary gland transplants, suggesting a developmental dependency for mammary carcinogenesis induction by DMBA. This may hold true for other PHCs as well.

In addition, efficiency of proper mammary gland development in the interscapular fat pad was retarded at the 40-45 day DMBA window. This implies that DMBA may have an effect on mammary gland development. At this time point, DMBA was administered only ten days following cell injections. DMBA causes acute immunotoxicity immediately after it is given (Gao et al., 2005). It may be that immune cells necessary for appropriate ductal branching and elongation are not present, resulting in atrophy of the mammary gland in the ectopic site. However, the high dose of DMBA administered to rats is not physiologically relevant; therefore, this effect is not applicable to human health but has implications on future rat studies. More studies will need to assess the mechanism by which DMBA affects mammary gland growth in these studies, e.g. measure immunological cells in the plasma and in the ectopic mammary gland.

The shift in the DMBA-susceptibility window suggests that at earlier stages of mammary gland development, DNA-damaging agents, such as PHCs, have less effect on mammary carcinogenesis. It is not clear what the implications of this are. These data may suggest a reduced role for mammary "stem" cells in progression to full carcinoma development. Cancer stem cells have been proposed to be principal mediators in primary 
tumor development and relapse following therapy. My data suggest, however, at earlier stages the stem cell niche may not be capable of driving full carcinogenesis and, rather, a more differentiated cell type is requisite for tumor development. Still, it could be argued that these undifferentiated cells may require more than a single dose of DMBA to develop frank carcinomas. Indeed, DMBA-induced carcinogenesis relies on steroidal hormone activation, such as estrogens, as a "second hit" to drive carcinogenesis and potentially these stem niche cells lack ample ER expression. We currently have no information regarding the cell populations present or how they act within the $60-65$ or 7075 day window. Therefore, there is no evidence for or against a role for a particular mammary gland cell type in DMBA-induced mammary carcinogenesis.

Previous studies have demonstrated that the clonogen transplantation method is sufficient to induce mammary gland formation in an ectopic site; yet, to date, no one has shown a capacity to stimulate mammary cancer development in these ectopic glands. These experiments are important because they provide insight into mammary carcinogenesis in the context of environmental exposures. Herein, I show that the mammary carcinogenic effects of DMBA are dependent on a specific stage of mammary gland development. Although the data imply that the $M c s I b$-conferred phenotype is not intrinsic to a mammary epithelial cell population, work to identify a specific cell type responsible for $M c s l b$-induced resistance, regrettably, remains inconclusive. Regardless, the initial goal of this aim was to identify a tissue type involved in $M c s l b$-conferred resistance to mammary carcinoma development. My data show that the action of the $M c s 1 b$ COP allele is specific to the mammary gland. Future efforts to functionally test gene candidates within the $M c s 1 b$ locus will, consequently, be focused on the mammary 
gland as a target tissue. Although these studies do not provide definitive answers as to what the specific factors are affecting tumor development, they afford a means by which to study these effects further. On the whole, my data identify that the Mcs $1 b$ COP allele is acting in mammary gland-autonomous manner and indicate that the effectiveness of DMBA to induce mammary carcinogenesis is dependent on the developmental stage of the mammary gland. 


\section{CHAPTER IV}

\section{MIER3 IS A CANDIDATE BREAST CANCER-ASSOCIATED GENE}

\section{Introduction}

There are thirteen transcripts annotated within the $1.8 \mathrm{Mb}$ Mcs $1 \mathrm{~b}$ locus delineated by lines $\mathrm{N} 3, \mathrm{~W} 2, \mathrm{U} 2$, and $\mathrm{I} 4$ and six of these transcripts reside in the $1 \mathrm{Mb}$ region delineated by addition of line $1 \mathrm{~b}-11$ (Figure 5). All of these genes are also annotated to the human breast cancer-associated locus marked by SNP rs889312, and any combination of these transcripts could be the responsible gene(s) involved in Mcs lb COP-conferred mammary carcinoma resistance (Figure 8). The most plausible breast cancer candidate gene within this region is Map3kl. However, no direct evidence is available to support Map3kl as the causal gene. It is relevant, then, to test the transcripts annotated to this region. We hypothesize that a genotypic variant between $\mathrm{WF}$ and COP in the $M c s l b$ locus lies within the coding region of one or more of these transcripts and results in an amino acid change that gives rise to a differential mammary carcinoma susceptibility phenotype. This approach is important in that SNP haplotype maps in the rat are incomplete; thus, identifying variants will increase current knowledge of rat genetics and potentially identify causative SNPs. Many cancers are caused by detrimental mutations or variants that alter protein function and this may be the basis the $M c s 1 b$ phenotype.

All of the human SNPs tagged along with rs889312 lie outside of annotated transcribed genes (Figure 3). Consequently, it is possible that the inherited element(s) 
controlling the risk phenotype are non-coding regulatory elements. Indeed, many low penetrance alleles associated with disease phenotypes are now being found to involve non-coding variations. There may be additional SNPs not yet identified that lie in transcribed regions that may be causative; thus, it is important to first test for changes in the coding region.

To not bias the approach towards any specific transcript(s), I first set out to resequence the open reading frames (ORFs) of each transcript lying in the $1.8 \mathrm{Mb} \mathrm{Mcslb}$ locus as it was delineated from marker $A 12 \mathrm{v}$ (ch2:42364155) to marker al2oo (ch2:44210175). The mechanism by which the Mcs $1 b$ locus is controlling mammary carcinogenesis susceptibility is not clear; therefore, including all of the transcripts in this interval reduces the probability of missing the causative factor.

In the past decade there has been increasing interest in regulatory microRNAs (miRNAs) in cancer research. These miRNAs are short pieces of untranslated RNA transcribed by the cell that can recognize and bind specific target sequence sites on translated mRNAs and mark them for degradation. They regulate rormal processes within the cell and have been shown to be dysregulated in some cancers. Differential effects can result either through sequence variation in the miRNA target sequence or through alterations in the sequence or the expression of the miRNA itself. These miRNA target sequences are principally located in the 3' untranslated regions (UTRs) of the mRNA to be regulated. Therefore, in addition to resequencing the ORFs, I also resequenced the 3' UTRs of these transcripts.

Sequence differences between WF and COP sequence in any of the transcripts will be analyzed in silico to determine if the variation(s) disrupts the normal structure of 
the protein, e.g., results in an amino acid change or introduces a premature stop codon. It may be that variation between the two rat lines results in differences in stability of a transcript. Efforts will be focused on determining transcript expression levels between susceptible WF and resistant WF.COP line T and N3 rats. Conversely, if no variation is identified in the coding region of any of these transcripts, it will be tacit that the mechanism of the $M c s 1 b$-conferred phenotype is regulatory in nature. In this case, gene expression of all the transcripts will be tested. In each case, gene expression levels will be first assessed in mammary gland tissue as we found $M c s 1 b$ COP-conferred mammary carcinoma resistance to be autonomous to the mammary gland. Other tissues will be tested to establish whether the effect is mammary gland-specific.

\section{Design and Methods}

\section{Resequencing Mcs1b Transcripts}

Spleen or thymus tissue from WF/Hsd and WF.COP lines N3 and T rats was excised and total RNA was extracted using TriReagent (Molecular Research Center) and standard chloroform/isopropanol precipitation. RNA samples were treated with TURBO DNase (Life Technologies) to reduce DNA contamination and cDNA was made using Superscript III reverse transcriptase (Life Technologies). Sequences were not attainable from cDNA in some instances; therefore, genomic DNA, extracted from frozen spleen or liver tissues using standard phenol-chloroform/isopropanol precipitation, was used. Samples were PCR amplified using Accuprime HiFi Taq polymerase (Life Technologies) and, subsequently, cleaned with the QIAquick PCR Purification Kit (Qiagen) to remove unincorporated nucleotides. Amplified samples were sequenced using the BigDye 
Terminator v.3.1 Cycle Sequencing Kit (Life Technologies and purified with Agencourt CleanSeq magnetic beads (Beckman Coulter). These were submitted for analysis to the University of Louisville Center for Genetics \& Molecular Medicine DNA Core using an ABI PRISM 3130XL Sequence Detection System (Life Technologies). Primer sequences for amplifying and sequencing Mcs $1 b$ ORFs and 3'-UTRs are in Table 9. Nucleic acid sequences were submitted to NCBI/GenBank and assigned accession numbers JQ013728 through JQ013739.

\section{Gene Expression Assays}

Tissues were excised from WF/Hsd and WF.COP lines N3 and $\mathrm{T}$ rats that had been treated with DMBA or not and flash-frozen in liquid nitrogen. Total RNA was extracted with TRI-Reagent (Molecular Research Center) followed by standard chloroform/ethanol precipitation. To reduce possible solvent and DNA contamination RNA samples were further processed by a $1 / 10 \mathrm{v} / \mathrm{v} 3 \mathrm{M}$ sodium acetate and $2.5 \mathrm{x} v / \mathrm{v}$ $100 \%$ ethanol wash on ice for 10 minutes followed by $80 \%$ ethanol wash followed by Turbo DNase (Life Technologies) treatment. Total RNA quantity and quality were measured with a Nanodrop 1000 (Fisher Scientific) and a Bioanalyzer with RNA 6000 NanoChips (Agilent). cDNA was made by reverse transcription reactions using (20 $\mu \mathrm{l}$ f.v.) $1 \mu \mathrm{g}$ total RNA, $0.5 \mathrm{x}$ RNAsecure, $5 \mu \mathrm{M}$ random hexamers, $25 \mathrm{ng} / \mu \mathrm{L}$ oligo $\left(\mathrm{dT}_{18}\right)$, and $0.5 \mathrm{mM}$ dNTPs were incubated 5 minutes at $65^{\circ} \mathrm{C}$ prior to adding $1 \times$ first strand buffer, $100 \mathrm{mM}$ DTT, and $1 \mu \mathrm{L}$ Superscript III (Life Technologies). Reactions were incubated 5 $\mathrm{m}$ at $25^{\circ} \mathrm{C}, 1 \mathrm{~h}$ at $50^{\circ} \mathrm{C}$, and $15 \mathrm{~m}$ at $70^{\circ} \mathrm{C}$. TaqMan QPCR primers and MGB probes (Applied Biosystems) were designed by using Primer Express v 2.0 (Applied 
rubic 9

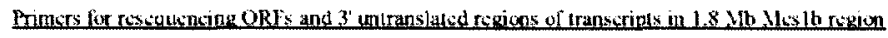

\begin{tabular}{|c|c|c|c|c|c|}
\hline $\begin{array}{c}\text { Gene } \\
\text { (GienBank Arression \#) }\end{array}$ & $\begin{array}{l}\text { Cenkank accession } \\
\text { WF and } C O P \\
\text { ref sequence }\end{array}$ & $\begin{array}{l}\text { Implifincation Primer } \\
\text { Lab ID }\end{array}$ & Ansplification Primer & $\begin{array}{l}\text { Nested Sequencing Primer } \\
\text { I,ab ID }\end{array}$ & Nested Primer \\
\hline Thap3ki & 10013733 & Map36:1-F14 & 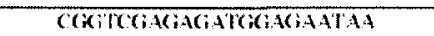 & Map3K-1-SqI & ACTCAAKCOACCTETAK \\
\hline \multirow[t]{5}{*}{ (AV $(15.1842)$} & & Wapik $1-R I$ & Moceacicateratcanci & Map.3k1.Sq2 & CTGACTCC TTCAAACECOA \\
\hline & & & & Map,3k1-Sq! & COCCATCETATGGIALAAAAT \\
\hline & & & & Mapp $3 k 1-S y 4$ & CGACTTCGGACTTGIAAGTGA \\
\hline & & & & Map $3 k 1-S_{4} 5$ & GCTCGITGGCTGCTIATTCT \\
\hline & & & & Map $3 k$ 1-Sy6 & 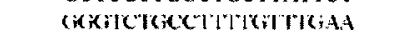 \\
\hline Niers & 10013732 & Mier3-Fi & ATGGCCACACCCCAOGTCOCAGTO & Micr3-Syl & CTGATCAGGAATGGOCTCAG \\
\hline \multirow[t]{3}{*}{ (NM 001168000$)$} & & Mier $3-\mathrm{RI}$ & TGAGAACGGTTCAACTTCC & Mier3-Syz & TCACCACTGTACTCGCCAAG \\
\hline & & & & Mier 3.54 ? & ACCCAGCALAGAACAAAGG \\
\hline & & & & Mier3-S44 & GAACATGCATGGATTTGTOC \\
\hline Giphpt & 50013734 & Gobpl-ti & AACACCTTCTOTOCAGTCC & Giplypl-Syl & CAGCATGOCTTCATCTICA \\
\hline (NM $00110 \times 410)$ & & Gplpl-R] & CATCTTCT LATGGGGTAGGTCA & (iphpl-Syz & OTGTGTOTC TGGGCOTGTAC: \\
\hline Inkrdss & $J(013731$ & WNKRDSS-F1-1 & GKGCACAWATGCLATHOCKG & WKRDISS-FZ-1 & GTITCOCOACGTCAGTGOA \\
\hline \multirow[t]{12}{*}{ (XM 342195) } & & ANKRDS5-R7-1 & AGCAGCAAACATGGCACAL & ANKRDS5-F3-1 & GAGGCTTCRCATCGICCTGCT \\
\hline & & & & INKRDSSFHA-1 & MGOTCCCCG MOTGT LACCTO \\
\hline & & & & WNKRDS-FS-1 & TGACATCATCGCCACCTTTO \\
\hline & & & & WKRDSS.FG.1 & TCAGALLOCCGLACGAC \\
\hline & & & & WNKRDSS-F7-1 & 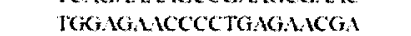 \\
\hline & & & & INKRDS5-F-1 & TGIGCOATGTTRGCTGCTIT \\
\hline & & & & MKRDSS-KI-I & 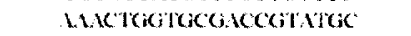 \\
\hline & & & & WKK155-k2-1 & 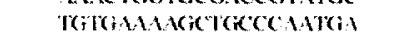 \\
\hline & & & & ANRK1DS5-K3-1 & 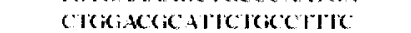 \\
\hline & & & & WKRDDS $-k-4-1$ & 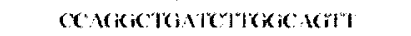 \\
\hline & & & & WKKDSS-RS-1 & 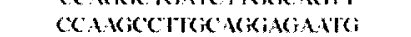 \\
\hline & & & & AVhR1555-116-1 & 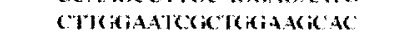 \\
\hline $116 \mathrm{se}$ & $1(x) 13730$ & Ilest mRVA a amplifi star $f$ & Miningidiclitiachetrilla & $116 s t+2-1$ & 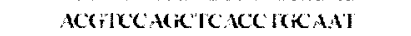 \\
\hline \multirow[t]{13}{*}{ MV omo0x725) } & & 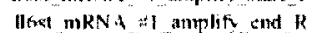 & 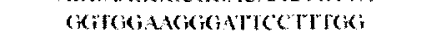 & If $6: 1-73-1$ & 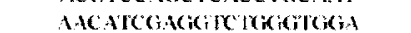 \\
\hline & & & & II $6 s_{1-1}: 4 \mid$ & 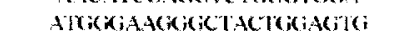 \\
\hline & & & & II 6 si-Fs-1 & THEACACCOCCATCT AAACC \\
\hline & & & & ILost-Fon-1 & GGTCCACATGGCAGCATACA \\
\hline & & & & $116 \times 1-1-7-1$ & COCCCAAGICACAATTITAAC \\
\hline & & & & a $6 \times 1 \cdot R 2-1$ & GGTGOEOTGGGTTTCACTIT \\
\hline & & & & 12.6st-R3-1 & GAAACTROGCGCTTKGATT; \\
\hline & & & & II 6 -6st-R4=1 & TCTGGGATGCAGGGTGAGTT \\
\hline & & & & Il ost-R5-1 & AAAGTGAATTCCOOCCCATC \\
\hline & & & & 1-6st-R6-1 & CCAAGGATTICAGOTCATCTGG \\
\hline & & & & $0.6 \mathrm{~s} 1-\mathrm{R} 7-1$ & CCTGOCTTGACCTTICGAACT \\
\hline & & & & likst mRNA $\$ 1$ amplify and $F$ & TRCTMGGCCCACACATCTCCA \\
\hline & & & & ll6st mRRA 1 anmplify start $R$ & CAAACCTOAATTGACCCATKC \\
\hline & $J 0013736$ & ||131 ra-mouseortho2-F| & TTCTCTAOCCTCOCTTCCAG & H.31R.L-F2-1 & CAGCTACACCGTGAGGATGACTT \\
\hline \multirow[t]{7}{*}{ (XXY 226759) } & & IL31R:-RT-1 & TCATTTCALGGGGCGTCTOT & IL-31RA-F3-1 & COALGGOTCTRCALCTCA \\
\hline & & & & n31RA-F4-1 & ACTOCTTCTOTTOOOCCAGA \\
\hline & & & & IL3IRA-15-1 & AOCOANTECATCENGCTATO \\
\hline & & & & II $31 \mathrm{R} A-\mathrm{F} 6-1$ & CKGGLACCALCCGATTOA \\
\hline & & & & $1131 \mathrm{R} \lambda \mathrm{N}-1 ; 7.1$ & 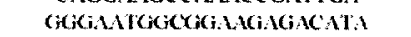 \\
\hline & & & & II3IRA-R2-1 & Tetrowecorrectecti \\
\hline & & & & II.31R-A-R3-1 & AACTETOCACATCACCACCA \\
\hline
\end{tabular}


Tables 9 antimued

\begin{tabular}{|c|c|c|c|c|c|}
\hline \multicolumn{6}{|c|}{ Table 9 untinued } \\
\hline 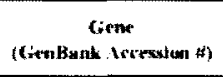 & 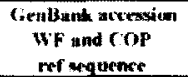 & $\begin{array}{l}\text { Ampluncattion Primer } \\
\text { 1,ant ID }\end{array}$ & Amplification Primer & $\begin{array}{l}\text { Yested Sequenetha Printr } \\
\text { 1.tab } 10\end{array}$ & Sinsted Primer \\
\hline & & & & IIIIRA-R4-1 & 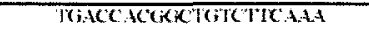 \\
\hline & & . & & $11,31 R A-R s-1$ & 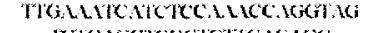 \\
\hline & & & & $1131 R A-R \theta-1$ & 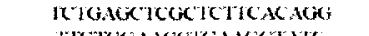 \\
\hline \multirow{14}{*}{ 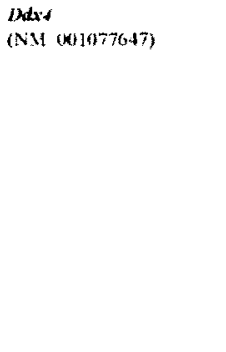 } & 10013737 & $x+9-1 x d x+\lambda=w-11$ & 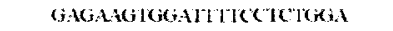 & $A 49-1) \mid x+x<w-54$ & 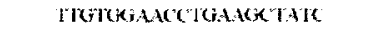 \\
\hline & & $D d X+R \&-1$ & 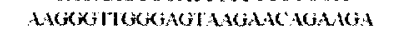 & (4) -1 dx+Now-ki & 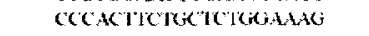 \\
\hline & & & & A4)-DUN-NGW-R2 & TCCACIAGCAAATCCACTICTC \\
\hline & & & & $149-1 \times 1 \times 4+N\left(m-R^{3}\right.$ & CATTCCATCTGQATACECO \\
\hline & & & & $1 \mathrm{ddx}+4 \cdot 3+1$ & ACKTGACACTCTOGTTCTOK \\
\hline & & & & $1 d x+1+1+1$ & DATACCCCTCCTCCACCAGit \\
\hline & & & & $10 \mathrm{dx} \times+\mathrm{F}-\mathrm{k}-1$ & 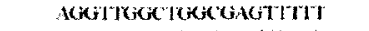 \\
\hline & & & & $1 . d x+1.7 .1$ & 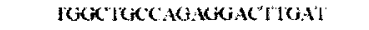 \\
\hline & & & & $D d x++F \times-1$ & 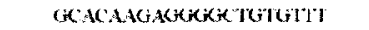 \\
\hline & & & & $n d x+R=-1$ & TOETKCATCATERCCAGATACTTC \\
\hline & & & & $D d x+R+1$ & KiTGCCCAACTGGOTTCCT \\
\hline & & & & $12 d \times 4 \cdot R s-1$ & 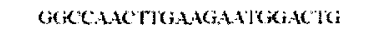 \\
\hline & & & & $D d x+R(n-1)$ & GCCC TGCCACTATTTCCACA \\
\hline & & & & $D A \times 4+R 7-1$ & 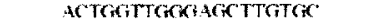 \\
\hline \multirow{5}{*}{$\begin{array}{l}\text { Ppap 2s } \\
\text { (NA1 022518) }\end{array}$} & $J(x) 1372 x$ & Ppap2arFil & 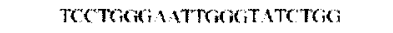 & P'ap 2a-R1-1 & TeT KeC Treactoc NGTT \\
\hline & & Ppap 2at-R+1 & TETTOGLAOCATTTTATATA & Ppap 2a+k2-1 & AGCOETRGAACCTTITTGT \\
\hline & & & & Prap2as Rz-1 & 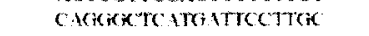 \\
\hline & & & & Prapiatis-1 & 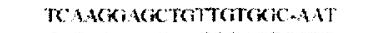 \\
\hline & & & & PpapzatR?-1 & 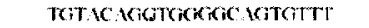 \\
\hline \multirow{10}{*}{ 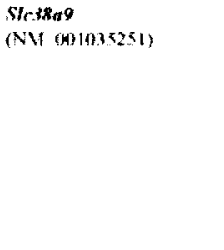 } & $9001 \times 729$ & $\operatorname{sic} 3 x+2 y+1.1$ & COACACICAAATCCOCATTC & $\operatorname{sic} 38.9-F 2-1$ & AGCYACATGCTOCTECt \\
\hline & & $\$ \$ 1<38 n \cdot R t+1$ & CAACATGCETTGACCCAACA & Slc:38ng-F:3-1 & TGCRGAAGTCCCGGIATT ATGA \\
\hline & & & & $\operatorname{Sic} 38 \times 9-\sqrt{4-1}$ & 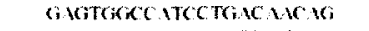 \\
\hline & & & & S163839-15-1 & TCCTGGTITTRCCTC.TTCC \\
\hline & & & & 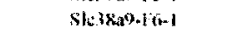 & 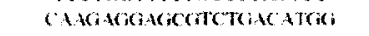 \\
\hline & & & & 51.3829 .21 .1 & 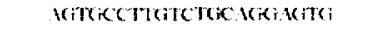 \\
\hline & & & & Ste?819.R2+1 & 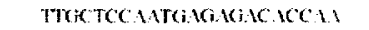 \\
\hline & & & & S1L38a9-R3-1 & 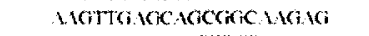 \\
\hline & & & & 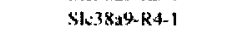 & 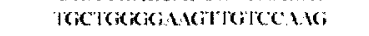 \\
\hline & & & & $\$ 1638 a 9-R \leq-1$ & TGCOACOTTCABACACA \\
\hline \multirow[t]{3}{*}{ Bap3k1-3"CTR } & & Map.31-31 TR-T! & 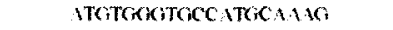 & & \\
\hline & & Map.3L1-31 TK-R1 & 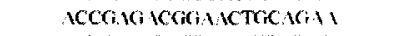 & & \\
\hline & & Stap 31 1-3t TK-R2 & 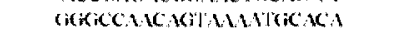 & & \\
\hline \multirow[t]{8}{*}{ Wiers-3ettA } & & Vier3-37TR-11 & ICGCKGCCTTCATTCKGK & & \\
\hline & & Mker?-3T TR-R & COTCTCOTCATTOCOCKCK & & \\
\hline & & Mier?-3l TR-12-2 & Mentacouceconce & & \\
\hline & & Mest3-3UTRE-R2-2 & 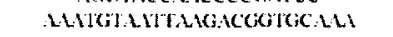 & & \\
\hline & & Stier $3-37$ TR-F2-3 & IOTOGLATCACATGTTTICAGG & & \\
\hline & & Mier,3-3UTR-R2-3 & 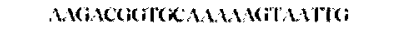 & & \\
\hline & & Vher3-3tTR-13 & 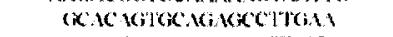 & & \\
\hline & & Mtore3.3LTR-K3 & 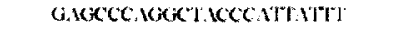 & & \\
\hline \multirow[t]{12}{*}{ Ciptopl-3tTK } & & (ippp 1-34 TRR-FI & cokrsectoctrouch & & \\
\hline & & (ippol-31 TR-12 & 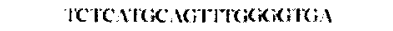 & & \\
\hline & & Giptpi-3UTR-13 & 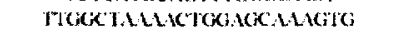 & & \\
\hline & & Gptp1-31/TR-F4 & 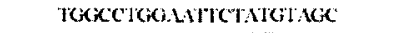 & & \\
\hline & & (jphp1-37 TR-15 & 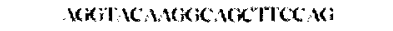 & & \\
\hline & & Gptep 1-3บTR-16 & 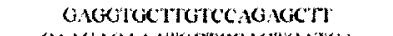 & & \\
\hline & & Cipppl-3I TR-RI & 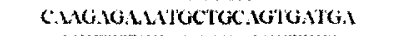 & & \\
\hline & & Gphpl-3LTR.R2 & AGCTCRGACAKGCOCTOCI & & \\
\hline & & Gpbpl-3l IR-R3 & 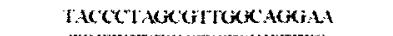 & & \\
\hline & & Giphpl-37 TR-R4-1 & 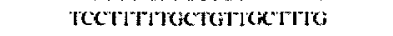 & & \\
\hline & & (iphpl-3U TR-R+2 & 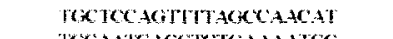 & & \\
\hline & & Gintpl-3T TR-Rs & TCCAAICACCICTGAAAATCC & & \\
\hline
\end{tabular}


Biosystems). Primer and probe sequences are provided in Table 10. One $\mu$ l of the dilution $(\approx 12.5 \mathrm{ng}$ of RNA-equivalent cDNA) was used in a 16- $\mu \mathrm{l}$ TaqMan QPCR. The reaction components were $1 \times T a q$ Man Buffer A (Applied Biosystems); $5.5 \mathrm{mM} \mathrm{MgCl}$; dATP, dCTP, dGTP, and dTTP at $400 \mu \mathrm{M}$ each; experimental primers at $300 \mathrm{nM}$ each; 200 nM TaqMan experimental probe (Applied Biosystems); Rplp2 primers at $100 \mathrm{nM}$ each, $200 \mathrm{nM}$ rodent Rplp2 probe; and 0.4 units of Taq Gold DNA Pol (Applied Biosystems). Real-time QPCR was run on an ABI PRISM 7900HT real-time PCR machine. Real-time QPCR cycling conditions were $50^{\circ} \mathrm{C}$ for $2 \mathrm{~min}$ and $95^{\circ} \mathrm{C}$ for $10 \mathrm{~min}$, followed by 40 cycles of $95^{\circ} \mathrm{C}$ for $15 \mathrm{sec}$ and $60^{\circ} \mathrm{C}$ for $1 \mathrm{~min}$. FAM (Mcs $1 \mathrm{~b}$ region target gene probe) and VIC (Rodent Rplp2 probe; Applied Biosystems) fluorescence values were measured by using Applied Biosystems SDS v 2.3 software; quantities of transcripts were measured by comparison of cycle threshold values with a standard curve calculated from serial dilutions. Sample measurements are an average of four replicates per sample and were standardized by dividing the quantity of rodent $R p l p 2$. Data were analyzed by Mann-Whitney tests.

\section{Comparative Genomics}

Human and mouse sequences for genes annotated to the Mcs $1 b$ locus were

obtained using the UCSC Genome Browser using the Homo sapiens version GRCh37/hg19, Rattus norvegicus version 3.4/rn4, and Mus musculus version NCBI37/mm9 genome assemblies. Sequences were aligned using the DNAStar SeqMan sequence analysis program (DNAStar) to identify orthologous regions. Primers were 
Table 10:

Rat Mcs lh Target and Rplp2 Primers and Probe Sequences Used for OPCR

\begin{tabular}{|c|c|c|c|c|}
\hline Assay Name & Forward Primer Sequence (5' to 3') & Reverse Primer Sequence (5' to 3') & Probe Sequence ( $5^{\prime}$ to $3^{\prime}$ ) & NCBI Gene Reference ID \\
\hline Rplp2 & TGAACGACTCAACAAGGTCATCA & CAACACCCTGAGCGATGACA & VIC-CTGAATGGAAAGAATATTGAG & NM 001030021.1 \\
\hline Map3kl & TCCTCATATTGTCAGTACCGATGTC & TTGCAAGGCAAAGGCTAAGAG & 6FAM-CCTGTTGAAATCAGGTATAA & NM 053887 \\
\hline Mier3 & CGAAAGGTACTGCTGTAATGGAAA & GCACTCCTCTTCAGTCCAAGCT & GFAM-CCTCTC AAGAAGGAATG & VM 001168000.1 \\
\hline Gobpl & GAGTAGAAGAGGAGCATGAAGATGAA & TGGTGAGTACTATTGCTGTTATGCAA & 6FAM-CTCAGAGAAGGATGACGAC & NM 001106410.1 \\
\hline IJ6st & GACAACGCTGCTGGGAGTCI & GGAGITAAAATTGTGCCTTGGG & 6HAM-AAACACATCRGGCCGAAT & NM_. 001008725.3 \\
\hline Ankrd55 & ATGGTGCCAAGCACAACATC & GATGAGAAGCCTCACATCAGGTT & 6FAM-CAGATAAAAATGGCCGCCTG & $\mathrm{XM} 342195.4$ \\
\hline $1131 \mathrm{ra}$ & GCCAAAGAAAAAGCTCCAACAT & ACGGCTGTCTCAAACCAATG & 6FAM-TCCCAMTACCACGGTGAA & XM 226759.4 \\
\hline $\operatorname{Dd} \times 4$ & CTGGGAAGACGGCAGCTTT & GCAGTTATTCCATCCCTCATCATA & 6FAM-CTCTTGCCTATTTTOOCTC & NM 001077647.1 \\
\hline Slc389 & GGTTTGCGCCAACAGAATTT & CCCTGTCAGCTGTGG AACTG & 6FAM-TTGTACCAGAGATAAGAGC & NM 001035251.1 \\
\hline Ppap2a & TGTCAAGGGAATGAACAGAAGGT & AACAGCATGCAGTACATAGAGAATGA & 6FAM-AAGGCAGGTTGTCCTI & NM 022538.2 \\
\hline
\end{tabular}


designed against the rat orthologous sequence using Primer3 (Rozen and Skaletsky, 2000 ) and used to test for alternate start sites for $M c s 1 b$ transcripts in rat cDNA samples.

\section{Genomics and Statistical Analysis}

Mammary carcinoma multiplicity phenotypes were compared by nonparametric Mann-Whitney tests. Results from mammary gland grafting experiments were analyzed using logistic regression. Donor and recipient genotypes were incorporated as dependent variables. In independent models, graft site tumor outcome and grafting ability were used as independent variables. Quantitative PCR (QPCR) data were analyzed using ANOVAs with $\log _{2}$ (Target quantity/Rplp2 quantity) as the dependent variable. Independent variables for comparing mammary gland transcript levels were $M c s l b$ genotype and DMBA exposure. Mcs $l b$ genotype and tissue source were independent variables for mammary carcinoma and non-diseased mammary tissue QPCRs. Fisher's PLSD tests were used to compare groups following a significant F-test $(\alpha \leq 0.05)$. Statview software (SAS Institute) was used.

\section{Cloning of Mier3 Splice Variants}

Splice variants for rat Mier3 were amplified from susceptible and Mcs $1 b$ resistant rat mammary gland cDNAs by standard PCR and cloned into a pCR ${ }^{\circledR} 2.1-$ TOPO $^{\circledR}$ vector (Life Technologies) according to manufacturer specifications. Clones were transformed into chemically-competent DH5 $\alpha$ E. coli cells, plated on Miller's LB Agar (Amresco) plates containing $50 \mu \mathrm{g} / \mathrm{ml} \mathrm{Kanamycin} \mathrm{and} \mathrm{coated} \mathrm{with} 40 \mu \mathrm{l}$ of $40 \mathrm{mg} / \mathrm{ml} \mathrm{X}$-gal, and incubated at $37^{\circ} \mathrm{C}$ overnight. White colonies were picked from each plate and inoculated 
into individual wells of a 96 well plate containing $40 \mu 1$ Miller's LB broth (Amresco) and incubated for 2 hours while shaking. $0.2 \mu 1$ of inoculated media was amplified by PCR using M13 reverse and T7 promoter primers specific for flanking regions of the multiple cloning site within the $\mathrm{pCR}^{\circledR}$ 2.1-TOPO vector. PCR products were resolved on $1 \%$ agarose TBE gels and stained with SYBR Gold. Large and small bands corresponding to full-length and spliced Mier3, respectively, were counted for each sample. Analysis was performed on the proportion of full-length bands in the total number counted. Proportions were arcsine-transformed and analyzed by a student's t-test.

\section{Results}

Mcs1b Potential Candidate Open Reading Frame Sequences Yields Are Not Different Between WF and COP

As shown in Figure 5, rat Mcs $1 b$ was found to contain thirteen potential candidate gene transcripts as well as sequence orthologous to human $5 q 11.2$, a GWAS-identified breast cancer risk associated allele marked by SNP rs889312 (Easton et al., 2007). To prioritize potential candidates, I resequenced conserved protein coding ORFs that were within the $1.8 \mathrm{Mb}$ interval that delimited $M c s l b$, and based on RT-PCR gel electrophoresis, were expressed in mammary glands of susceptible WF and $M c s l b$ resistant females (lines N3 and T). Transcripts from Gpbp1, Map3k1, Mier3, Ankrd55, Il6st, Il31ra, Ddx4, Slc38a9, and Ppap2a genes were detected in mammary gland total RNA pools from each genotype by RT-PCR. No genetic variants were identified between susceptible WF and $M c s I b$ resistant genotype ORFs or 3' UTRs for these transcripts. Nucleotide sequences were submitted to NLM-NCBI and the GenBank 
accession numbers are provided in Table 9.

Four of the $M c s l b$ candidate genes are predicted transcripts based on sequence containing gene-coding properties, e.g. intron-exon boundaries and polyadenylation signals, or sequence similarity to known expressed transcripts: Actbl2, ENSRNOG00000013098, C5orf35 and U6 snRNA (labeled with asterisks in Figure 5). Rat $A c t b l 2$ was identified as a pseudogene and is located outside rat genomic sequence orthologous to the human $5 q 11.2$ haplotype block that associates with breast cancer risk. Predicted transcript ENSRNOG00000013098 was listed on the Ensembl genome browser (Flicek et al., 2011). I found no evidence by RT-PCR of a transcript from Actbl2 or ENSRNOG00000013098 in total RNA samples from multiple susceptible and Mcslb resistant mammary glands or in rat mixed tissue total-RNA samples that included embryo, brain, testes, ovary, thymus, spleen, and liver. Since cDNA was not attainable and $A c t b l 2$ was predicted to be a single-exon transcript, we sequenced genomic DNA spanning this predicted pseudogene and found no sequence differences between WF and COP alleles.

Rat C5orf35 is an ortholog of human C5ORF35. This gene was recently identified to have a SET domain and renamed SETD9 (SET domain containing 9). SET family members are histone methyltransferases involved in regulating chromatin structure by methylation of lysine residues on tails of histones. Dysregulation of SET proteins plays roles in the progression of many cancer types. For instance, the SET member $E Z H 2$ has been implicated in the development of breast and prostate cancer (Kleer et al., 2003; Yu et al., 2007). Although the human C5ORF35 protein has not been fully characterized it could be a potential candidate based on its sequence similarity to 
SET proteins. However, rat C5orf35 was not present in any total RNA samples tested from various rat tissues. Additionally, I successfully amplified C5ORF35 from human thymus, spleen, and ovary, but not human breast tissue cDNA (Figure 16A) suggesting that human C5ORF35 is not highly expressed in the breast. However, in an Oncomine (Rhodes et al., 2004) database search I found that other groups have reported detection of

C5ORF35 in human breast carcinoma and non-diseased breast tissue. Using bioinformatic analysis I discovered that the annotated 5'- and 3'- UTRs of human C5ORF35 are poorly conserved between humans and rodents (Figure 16B); therefore, we concluded that C5ORF35 is a human, but not a rat transcript.

A predicted small nuclear RNA (snRNA) at rat position Chr2:4376581143765918 named U6 or ENSRNOG00000034909 is estimated to be 108 bp on the forward strand. I noted that ENSRNOG00000034909 sequence aligned to approximately 100 distinct regions of the rat genome using both NCBI/BLAST and UCSC/BLAT (den Dekker et al., 2012; van Boxtel et al., 2011) (Table 11). Because of the highly repetitive nature of the sequence, I was unable to design specific probes to determine if this predicted single exon gene was transcribed from rat $M c s l b$.

\section{Mcs1b Potential Candidate Gene Expression Levels Uncover Mier3 as a Potential Gene Candidate}

Rat $M c s 1 b$ did not contain any protein coding genetic variation between $M c s l b$ susceptible and resistant alleles; therefore, rat $M c s l b$ may contain variation in one or more non-protein-coding regulatory elements that differentially control gene expression between mammary cancer-susceptible and resistant genotypes. To test this hypothesis, I 


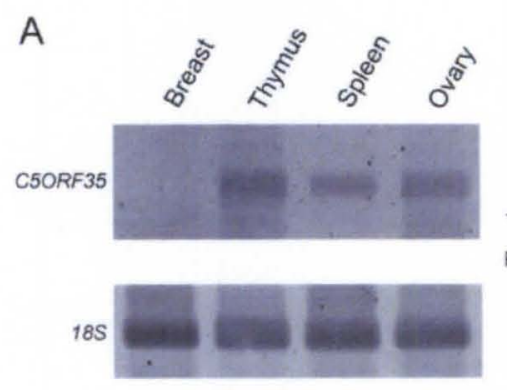

B

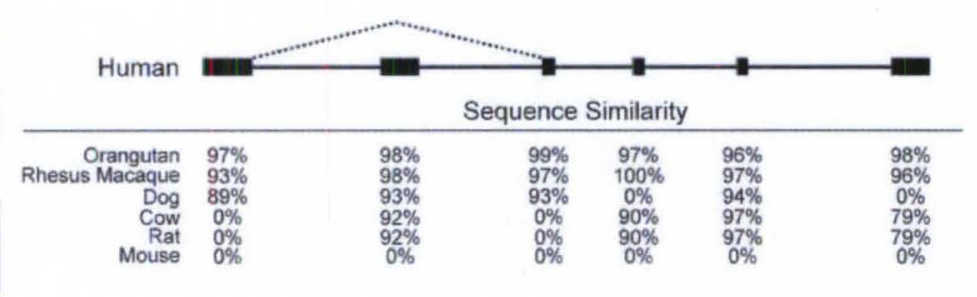

Figure 16: Predicted human C5ORF35 is not conserved in rats. A; Predicted C5ORF35 transcript was detected in multiple human tissues by RT-PCR. B; Splice variants of C5ORF35 detected in human tissue and sequence similarity between human C5ORF35 exons and other species. A C5ORF35 splice variant that did not contain exon 2 was detected in human thymus and spleen samples. C5ORF35 is not conserved between primates and rodents based on degeneration of 5' and 3'UTR sequences in rodents. 
Table 11:

BLAT alignment of ENSRNOG00000034909 sequence against UCSC Genome Browser Rat DNA build (Bavlor 3.4m4 assembly)

\begin{tabular}{|c|c|c|c|c|c|c|c|c|}
\hline Transcript ID & Transcript Description & Chromosome & Start Position & End Position & Orientation & Score & $\%$ ID & Span (bps) \\
\hline No rat transcripts annotated in browser & & Chr:2 & 43765811 & 43765918 & + & 108 & 100.00 & 108 \\
\hline RefSeq ID: NM 153738 & Rat Pripn intronic region & Chr: $1^{-}$ & 43789233 & 43789333 & $=$ & 82 & 88.20 & 101 \\
\hline No rat transcripts annotated in browser & & Chr:o & 136766481 & 136766580 & - & 79 & 88.70 & 100 \\
\hline RefSeq ID: NM 001 108397.1 & Rat $S$ frs 14 intronic region & Chr:lo & 19667327 & 19667415 & - & 78 & 93.10 & 89 \\
\hline RefSeq ID: NM 001191653.1 & Rat Tanc2 intronic region & Chr: 10 & 95198700 & 95198796 & - & 78 & 89.40 & 97 \\
\hline No rat transcripts annotated in browser & & Chr:- & 98699371 & 98699471 & - & 77 & 91.10 & 101 \\
\hline RefSeq ID: NM 173101.1 & Rat Myole intronic region & Chr:s & 74882368 & 74882468 & - & 75 & 89.90 & 101 \\
\hline No rat transcripts annotaled in browser & & Chr: & 110618884 & 110618978 & - & 75 & 92.80 & 95 \\
\hline No rat transcripts annotated in browser & & Chr:ll & 63198076 & 63198175 & - & 75 & 89.70 & 100 \\
\hline No rat transeripts annotated in browser & & Chr:l & 103440155 & 103440263 & $\therefore$ & 75 & 90.50 & 109 \\
\hline No rat Iranscripts annotated in browser & & Chr:l & 75525663 & 75525763 & 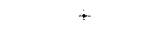 & 75 & 89,90 & 101 \\
\hline No rat transeripts annotated in browser & & Chr:3 & 69993031 & 69993128 & - & 74 & 90.70 & 98 \\
\hline No rat transcripts annotaled in browser & & Chr:2 & 19495997 & 19496085 & - & 74 & 90.70 & 89 \\
\hline No rat transcripts annotated in browser & & Chr:l & 245228475 & 245228564 & . & 74 & 91.20 & 90 \\
\hline RefSeq ID: NM 031337 & Rat Siat9 intronic region & Chr:4 & 105395193 & 105395722 & $=$ & 73 & 95.20 & 530 \\
\hline No rat transcripts annotated in browser & & Chr:- & 79649136 & 79649236 & & 73 & 88.80 & 101 \\
\hline No rat transeripts annotated in browser & & Chr:20 & 29978369 & 29978469 & 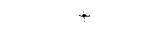 & 73 & 88.80 & 101 \\
\hline No rat transeripts annotated in browser & & Chr:l & 25409944 & 25410031 & . & 73 & 90.60 & 88 \\
\hline RefSeq ID: NM 001011915 & Rat Plekhc1 intronic region & Chr:15 & 21384589 & 21384682 & ; & 73 & 88.80 & 94 \\
\hline RefSeq ID: NM 001011915.1 & Rat Fermt 2 intronic region & $" \quad "$ & $" \quad "$ & $" \quad "$ & '"' & $m "$ & $"$ & " " \\
\hline No rat transeripts annotated in browser & & Chr:2 & 173740365 & 173740453 & ; & 72 & 89.60 & 89 \\
\hline No rat transcripts annotated in browser & & Chr:9 & 112012955 & 112013153 & t. & 71 & 94.00 & 199 \\
\hline No rat transcripts annotated in browser & & $C h r: 2$ & 124284322 & 124284416 & . & 71 & 90.40 & 95 \\
\hline No rat transcripts annot ated in browser & & Chr:ll & 70013852 & 70013937 & + & 71 & 90.40 & 86 \\
\hline RefSeq ID: NM 001135761.1 & & Chr:3 & 17548151 & 17548246 & - & 70 & 93.90 & 96 \\
\hline No rat transcripts annotated in browser & & Chr:? & 135345735 & 135345826 & + & 70 & 88.10 & 92 \\
\hline No rat transcripts annotated in browser & & Chr: 18 & 27228793 & 27228894 & . & 70 & 86.70 & 102 \\
\hline No rat transeripts annotated in browser & & Chr: 8 & 31027110 & 31027202 & - & 69 & 90.20 & 93 \\
\hline No rat transcripts annotated in browser & & Chr:5 & 15608653 & 15608741 & - & 69 & 92.30 & 89 \\
\hline No rat transcripts annotated in browser & & Chr:2 & 240633692 & 240633780 & - & 69 & 92.30 & 89 \\
\hline Rat mRNA from GenBank, ID: BC166504 & Rat Faml 89a2 intronic region & Chr:I & 227408075 & 227408163 & - & 69 & 92.30 & 89 \\
\hline No rat transcripts annotated in browser & & Chr:l & 18291369 & 18291554 & . & 69 & 92.70 & 186 \\
\hline RefSeq ID: NM 001170548.1 & Rat Thumpd 3 intronic region & Chr:A & 148933048 & 148933143 & + & 68 & 85.60 & 96 \\
\hline Rat mRNA from GenBank, ID: $\mathrm{FQ} 230829$ & No identified bomolog & Chr:19 & 41214051 & 41214136 & + & 68 & 84.40 & 86 \\
\hline No rat transeripts annotated in browser & & Chr:14 & 18935943 & 18936031 & ' & 68 & 87.30 & 89 \\
\hline No rat transcripts annotaled in browser & & Chr:9 & 47499507 & 47499595 & - & 67 & 91.00 & 89 \\
\hline
\end{tabular}


Table 11 continued

\begin{tabular}{|c|c|c|c|c|c|c|c|c|}
\hline Transeript ID & Transeript Description & Chromosome & Start Position & End Position & Orientation & Score & \%ID & Span (bps) \\
\hline RefSeq ID: NM 001197907.1 & Rat Oxrl intronic region & Chr:- & 77197714 & 77197802 & - & 67 & 91.00 & 89 \\
\hline RefSeq ID: NM 001171177.2 & Rat Tmtc2 intronic region & $\mathrm{Chr}: ?$ & 43891984 & 43892076 & - & 67 & 84.50 & 93 \\
\hline No rat transcripts annotated in browser & & Chr: 7 & 11235427 & 11235515 & - & 67 & 91.00 & 89 \\
\hline RefSeq ID: NM 031057 & Rat Aldh6al intronic region & Chr:6 & 108710588 & 108710676 & - & 67 & 91.00 & 89 \\
\hline No rat transcripts annotated in browser & & Chr:6 & 93546629 & 93546717 & - & 67 & 91.00 & 89 \\
\hline No rat transcripts annotated in browser & & Chr:6 & 72766763 & 72766851 & - & 67 & 91.00 & 89 \\
\hline RefSeq ID: NM 001134463.1 & Rat Cankmt intronic region & Chr:t & 8704054 & 8704142 & - & 67 & 91.00 & 89 \\
\hline No rat transcripts annotated in browser & & Chr:s & 151444756 & 151444844 & - & 67 & 91.00 & 89 \\
\hline RetSeq ID: NM 001108005.1 & Rat Mast 2 intronic region & Chr:5 & 136630633 & 136630721 & - & 67 & 91.00 & 89 \\
\hline No rat transeripts annotated in browser & & Chr:5 & 60215398 & 60215486 & - & 67 & 91.00 & 89 \\
\hline No rat transcripts annotated in browser & & Chrs & 56854404 & 56854492 & - & 67 & 91.00 & 89 \\
\hline No rat transcripts annotated in browser & & Chr:3 & 36733997 & 36734085 & - & 67 & 91.00 & 89 \\
\hline No rat transcripts annotaled in browser & & Chr:? & 35650885 & 35650973 & - & 67 & 91.00 & 89 \\
\hline No rat transcripts annotated in browser & & Chr:2 & 233090377 & 233090465 & - & 67 & 91.00 & 89 \\
\hline No rat transcripts annotated in browser & & Chr:2 & 168539521 & 168539609 & - & 67 & 91.00 & 89 \\
\hline RefSeq ID: NM 001034961 & Rat Sohlh2 intronic region & Chr:2 & 144422171 & 144422259 & - & 67 & 91.00 & 89 \\
\hline No rat transcripts annotated in browser & & Chr:2 & 96996138 & 96996226 & - & 67 & 91.00 & 89 \\
\hline No rat transcripts annotated in browser & & Chr:2 & 35329196 & 35329284 & - & 67 & 91.00 & 89 \\
\hline No rat transcripts annotated in browser & & Chr: 18 & 13043912 & 13044000 & - & 67 & 88.90 & 89 \\
\hline RefSeq ID: NM 001004020.1 & Rat Tmprss 11 b intronic region & Chr:lt & 23014788 & 23014876 & - & 67 & 91.00 & 89 \\
\hline No rat transcripts annotated in browser & & Chr:14 & 9590393 & 9590481 & - & 67 & 91.00 & 89 \\
\hline No rat transcripts annotated in browser & & Chr:10 & 60526129 & 60526217 & - & 67 & 91.00 & 89 \\
\hline ReISeq ID: NM 001191634.1 & Rat Gbfl intronic region & Chr:l & 251394207 & 251394295 & - & 67 & 91.00 & 89 \\
\hline Rat mRNA from GenBank, ID: FQ232940 & No identified homolog & Chr:l & 23671516 & 23671604 & - & 67 & 91.00 & 89 \\
\hline No rat transcripts annotated in browser & 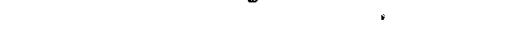 & $(h r)$ & 18892950 & 18893050 & - & 67 & 85.40 & 101 \\
\hline RefSeq ID: NM 012774.1 & Rat Gpe3 intronic region & Chr:X & 139315241 & 139315329 & + & 67 & 91.00 & 89 \\
\hline No rat transcripts annotated in browser & & $C h r:-$ & 127637150 & 127637238 & . & 67 & 91.00 & 89 \\
\hline No rat transcripts annotated in browser & & Chr:6 & 72766085 & 72766173 & $=$ & 67 & 91.00 & 89 \\
\hline RefSeq ID: NM 001134628.1 & Rat RGD1564943 (Predicted) intronic region & Chr:5 & 148336835 & 148336923 & + & 67 & 91.00 & 89 \\
\hline No rat transcripts annotated in browser & & Chr:A & 102436898 & 102436986 & 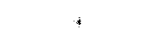 & 67 & 91.00 & 89 \\
\hline RerSeq ID: NM 022217.1 & Rat Amph intronic region & Chr:l? & 53682453 & 53682541 & + & 67 & 91.00 & 89 \\
\hline No rat transcripts annotated in browser & & Chr:l- & 48877826 & 48877914 & + & 67 & 91.00 & 89 \\
\hline No rat transcripts annotated in browser & & Chr:lt & 111622041 & 111622131 & 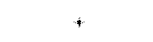 & 67 & 89.90 & 91 \\
\hline No rat transcripts annotated in browser & & Chr:10 & 89929380 & 89929468 & ; & 67 & 91.00 & 89 \\
\hline No rat transcripts annotated in browser & & Chr:X & 132443461 & 132443547 & + & 66 & 91.00 & 87 \\
\hline RefSeq ID: NM 001106706.1 & Rat Ttc27 intronic region & Chr: 6 & 20562368 & 20562456 & - & 65 & 89.70 & 89 \\
\hline No rat transcripts annotated in browser & & Chr: & 26126909 & 26126997 & - & 65 & 91.80 & 89 \\
\hline No rat transcripts annot ated in browser & & Chr:2 & 216457973 & 216458061 & - & 65 & 89.70 & 89 \\
\hline No rat transeripts annotated in browser & & Chr:ls & 33879457 & 33879556 & $=$ & 65 & 92.40 & 100 \\
\hline
\end{tabular}


Table 11 continued

\begin{tabular}{|c|c|c|c|c|c|c|c|c|}
\hline Transcript ID & Transcript Description & Chromosome & Start Position & End Position & Orientution & Score & \%1D & Span (bps) \\
\hline RefSeq ID: NM 001007145 & Rat Catnal intronic region & Chr:ls & 27639818 & 27639906 & - & 65 & 89.70 & 89 \\
\hline No rat transcripts annotated in browser & & Chr:lo & 265348 & 265436 & - & 65 & 89.70 & 89 \\
\hline No rat transcripts annotated in browser & & Chr:l4 & 40179238 & 40179338 & - & 65 & 84.30 & 101 \\
\hline No rat transcripts annotated in browser & & Chr:ll & 32577174 & 32577262 & - & 65 & 89.70 & 89 \\
\hline No rat transcripts annotated in browser & & Chr:? & 113696949 & 113697045 & . & 65 & 92.30 & 89 \\
\hline No rat transcripts annotated in browser & & $C h r: 2$ & 190279006 & 190279094 & : & 65 & 89.70 & 89 \\
\hline No rat transcripts annotated in browser & & chr:ls & 16341735 & 16341823 & + & 65 & 89.70 & 89 \\
\hline No rat transcripts annotated in browser & & Chr: & 231873185 & 231873266 & + & 65 & 86.60 & 82 \\
\hline Rat mRNA from GenBank. ID: FQ227174 & No identified homolog & Chr: & 15279892 & 15279979 & - & 64 & 89.50 & 88 \\
\hline No rat transcripts annotated in browser & & Chr:lt & 77213446 & 77213534 & - & 64 & 89.50 & 89 \\
\hline Rat mRNA from GenBank. ID: DQ100481 & INNE-1 retrotransposon nucleic acid binding protein mRNA & Chr:l & 25793445 & 25793533 & - & 64 & 84.10 & 89 \\
\hline No rat transcripts annotated in browser & & Chr:o & 116937744 & 116937821 & $:$ & 64 & 91.10 & 78 \\
\hline No rat transeripts annotated in browser & & Chr:lt & 32819567 & 32819654 & - & 63 & 89.10 & 88 \\
\hline RefSeq ID: NM 001107047.1 & Rat lkzt3 intronic region & (hr: 10 & 87362236 & 87362320 & - & 63 & 90.50 & 85 \\
\hline RefSeq ID: NM 001007630.1 & Rat $\angle$ fand 6 intronic region & Chr:l & 140906354 & 140906442 & - & 63 & 88.40 & 89 \\
\hline No rat transeripts annotated in browser & & Chr:I & 56584008 & 56584096 & - & 63 & 88.40 & 89 \\
\hline No rat transcripts annotated in browser & & Chr:9 & 103107997 & 103108085 & . & 63 & 88.40 & 89 \\
\hline No rat transcripts annotaled in browser & & Chr:3 & 104572110 & 104572198 & + & 63 & 88.40 & 89 \\
\hline No rat transeripts annotated in browser & & chr:l & 194118835 & 194118923 & ; & 63 & 88.40 & 89 \\
\hline RefSeq ID: NM 001135718.1 & Rat Prexl intronic region & Chr:3 & 157849079 & 157849155 & + & 62 & 89.20 & 77 \\
\hline No rat transuripts annotated in browser & & Chr:/6 & 36604669 & 36604747 & - & 61 & 91.40 & 79 \\
\hline No rat transeripts annotated in browser & & Chr:It & 54341337 & 54341425 & - & 60 & 90.00 & 89 \\
\hline No rat transcripts annotated in browser & & Chr:l & 232461672 & 232461742 & - & 60 & 91.20 & 71 \\
\hline RefSeq ID: NM 001191669.1 & Rat Thsd7b intronic region & Chr:ls & 42749088 & 42749194 & $\therefore$ & 60 & 97.00 & 107 \\
\hline
\end{tabular}

(Hits with score $\geqslant 60$ ) 
measured mammary gland transcript levels of genes located at Mcs Ib in 12-week old virgin female rats that were exposed to DMBA at 50-55 days and age matched controls without DMBA. These studies focused on mammary gland transcript levels due to the mammary gland autonomous nature of $M c s 1 b$ previously discussed. Twelve-week old animals were used because this is the age after the acute phase for DMBA-toxicity and before induced frank mammary carcinomas are detectable in susceptible strains.

Differences in expression between genotypes were analyzed by non-parametric Mann-Whitney tests and the effects of Mcslb genotype and DMBA exposure on candidate gene transcript levels were analyzed by two-way ANOVA (Table 12). Transcript levels of Ankrd55, Il3Ira, Ddx4, Slc38a9, or Ppap2a were not significantly different between $M c s 1 b$ resistant and susceptible mammary glands exposed to DMBA or not. However, the effect of $M c s 1 b$ genotype was statistically significant $(\mathrm{P}<0.05)$ for Gpbp1, Mier3, and Map3k1. Furthermore, there was a significant effect of DMBA exposure on Map $3 k 1$ transcript levels $(\mathrm{P}=0.0003)$ and the interaction between $M c s 1 b$ genotype and DMBA exposure approached statistical significance for Map3k1 $(\mathrm{P}=0.0588)$. Additionally, there was an effect of $M c s 1 b$ genotype on Il6st expression by ANOVA; however, there was no difference in expression between genotypes in mammary glands not exposed to DMBA $(\mathrm{P}=0.1137)$ and expression only approached significance in glands exposed to DMBA $(\mathrm{P}=0.0734)$. When $M c s 1 b$ genotypes were compared by DMBA exposure, mammary gland transcript levels were significantly different for Gpbpl, Mier3, and Map3k1 between Mcslb resistant and susceptible mammary glands that were not exposed to DMBA. However, significant expression differences between $M c s l b$ resistant and susceptible genotypes were sustained only for 


\section{Table 12:}

Analysis and statistics of $M c s 1 b$ potential candidate gene mammary gland transcript levels in $M c s 1 b$-resistant and susceptible genotypes at 12 weeks of age

Two-way ANOVA $F$ test $P$ values

\begin{tabular}{|c|c|c|c|c|c|c|c|}
\hline \multirow[b]{2}{*}{ Target } & & \multirow[b]{2}{*}{ Exposure } & & \multirow[b]{2}{*}{ P-value ${ }^{a}$} \\
\hline & Mcs1b Genotype & Exposure & $\mathbf{G X E}$ & & Susceptible & $M c s 1 b$ Resistant & \\
\hline \multirow[t]{2}{*}{ Gpbpl } & 0.0101 & 0.209 & 0.6422 & Control & $0.586 \pm 0.600(34)$ & $0.044 \pm 0.734(29)$ & 0.002 \\
\hline & & & & DMBA & $0.281+1.309(45)$ & $0.097 \pm 1.246(42)$ & 0.1716 \\
\hline \multirow[t]{2}{*}{ MIER3 } & 0.0023 & 0.7911 & 0.6682 & Control & $0.115 \pm 0.594$ & $-0.522+1.278(34)$ & 0.0104 \\
\hline & & & & DMBA & $0.154 \pm 1.557(45)$ & $0.688 \quad 1.943(48)$ & 0.024 \\
\hline \multirow[t]{2}{*}{ Map3kl } & 0.0002 & 0.0003 & 0.0588 & Control & $-0.092 \pm 0.818(34)$ & $-0.725 \pm 0.767(32)$ & 0.0019 \\
\hline & & & & DMBA & $0.105+0.564(47)$ & $-0.104 \pm 0.651(45)$ & 0.1036 \\
\hline \multirow[t]{2}{*}{ Ankrd55 } & 0.4694 & 0.2025 & 0.9019 & Control & $-0.691 \pm 0.678(24)$ & $-0.826 \pm 1.108(22)$ & 0.618 \\
\hline & & & & DMBA & $-0.377 \pm 1.296(17)$ & $-0.567 \pm 1.006(22)$ & 0.609 \\
\hline \multirow[t]{2}{*}{ Il6st } & 0.0199 & 0.1744 & 0.8435 & Control & $-0.066 \pm 0.755(36)$ & $-0.418 \pm 1.054(33)$ & 0.1137 \\
\hline & & & & DMBA & $0.189 \pm 1.006(44)$ & $-0.227 \pm 1.181(48)$ & 0.0734 \\
\hline \multirow[t]{2}{*}{ Ilsira } & 0.2869 & 0.8674 & 0.9928 & Control & $-0.331+1.072(24)$ & $-0.559+0.761(23)$ & 0.4072 \\
\hline & & & & DMBA & $-0.368 \pm 0.942(20)$ & $-0.592 \pm 1.159(23)$ & 0.4949 \\
\hline \multirow[t]{2}{*}{$D d x 4$} & 0.0555 & 0.5442 & 0.4045 & Control & $-0.107 \pm 0.983(36)$ & $0.359 \pm 0.911(33)$ & 0.2748 \\
\hline & & & & DMBA & $-0.055 \pm 1.122(18)$ & $-0.690 \pm 1.575(17)$ & 0.1769 \\
\hline \multirow[t]{2}{*}{ Slc38a9 } & 0.1008 & 0.3929 & 0.973 & Control & $-0.285 \pm 0.600(24)$ & $-0.575 \pm 0.681(23)$ & 0.1284 \\
\hline & & & & DMBA & $-0.144 \pm 0.970$ & $-0.422 \pm 0.954(23)$ & 0.3499 \\
\hline \multirow[t]{2}{*}{ Ppap2a } & 0.3918 & 0.8314 & 0.5788 & Control & $-0.385 \pm 0.632(24)$ & $-0.447 \pm 0.765(23)$ & 0.7629 \\
\hline & & & & DMBA & $-0.315 \pm 1.357(20)$ & $-0.605 \pm 1.029(23)$ & 0.4315 \\
\hline
\end{tabular}

${ }^{a}$ Fisher PLSD test $P$-values from comparing susceptible and $M c s / b$-resistant genotypes by exposure. 
Mier3 when females given DMBA were compared between genotypes $(\mathrm{P}=0.024)$. Transcript levels of Gpbpl and Map3kl were not different between genotypes when DMBA-exposed females were evaluated.

Mammary gland transcript levels were lower in $M c s 1 b$ resistant genotype females

for all genes with a significant difference between genotypes: Gpbp1, Mier3, and Map3k1. Mier3 mean transcript levels were approximately 4.5-fold lower in Mcs $1 b$ resistant compared to susceptible genotype mammary glands whether animals were exposed to DMBA or not (Table 12). Thus, exposure to DMBA had no appreciable effect on Mier3 differences between susceptible and Mcs $I b$ resistant genotype females. No significant differences in Mier3 transcript levels were detected between $M c s / b$ resistant and susceptible genotypes in spleen, thymus, ovary, or brain tissues (Figure 17). This suggests that Mier3 transcript level differences between $M c s l b$ alleles may be specific to mammary gland tissue.

\section{Mier3 Is Expressed as Three Different Variants in the Rat Mammary Gland}

Gene expression studies identified Mier 3 as a potential rat mammary carcinoma susceptibility-related gene. Interestingly, I also noted that Mier3 migrated as 2 bands on electrophoretic gels suggesting that different variants of Mier3 may be being expressed; therefore, I sequenced these variants. Moreover, I also sought to check if these Mier3 variants were expressed in the mammary gland and whether they were expressed at the same level between WF and COP tissue.

Mier3 was cloned into a TOPO vector and many clones were sequenced. Sequencing showed that both Mier 3 variants were expressed in the mammary gland 


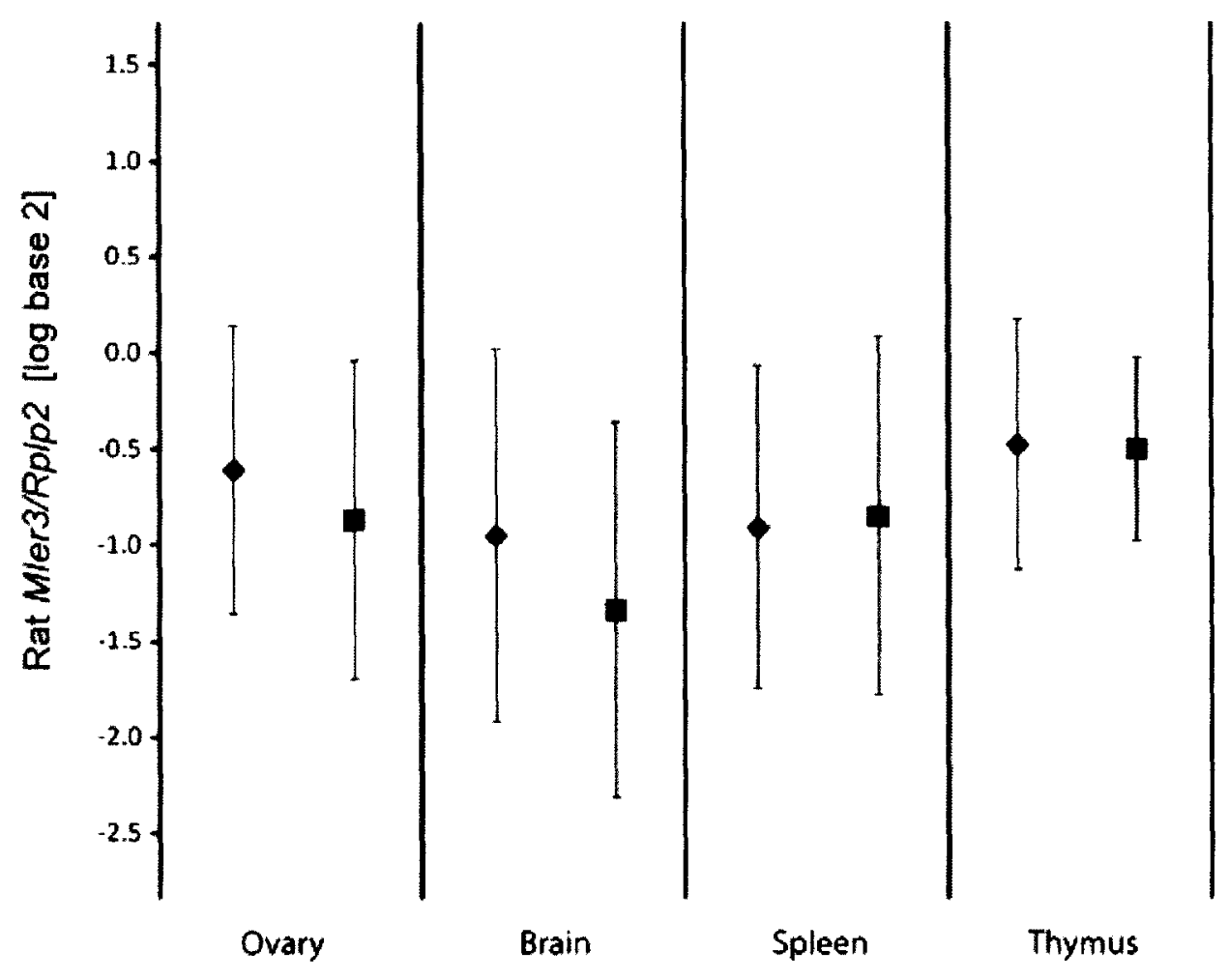

Figure 17: Mier3 is not differentially expressed between WF susceptible and Mcs Ib resistant WF.COP congenic females in rat ovary, brain, spleen, or thymus tissues. Expression (QPCR) levels are represented as $\log$ base 2 mean quantities of rat Mier3 relative to rat Rplp2 expression $+/-\mathrm{SD}$ for each tissue listed on the $\mathrm{X}$ axis. Comparisons between WF susceptible ( $\bullet$ ) and $M c s l b$ resistant $(\bullet)$ females for each tissue were not statistically significant in one-way ANOVA with post hoc Fischer's PLSD test (Ovary: $\mathrm{P}=0.4755, \mathrm{~N}=8 \mathrm{WF}, \mathrm{N}=13$ Mcs $1 b$ resistant line N3; Brain: $\mathrm{P}=0.3788, \mathrm{~N}=11 \mathrm{WF}, \mathrm{N}=10$ $M c s 1 b$ resistant line N3; Spleen: $\mathrm{P}=0.8854, \mathrm{~N}=8 \mathrm{WF}, \mathrm{N}=13 M c s 1 b$ resistant line N3; Thymus: $\mathrm{P}=0.9277, \mathrm{~N}=8 \mathrm{WF}, \mathrm{N}=12$ Mcs $1 b$ resistant line $\mathrm{N} 3$ ). 
(Figure 18A). One sequence identified the full length Mier3 transcript (Figure 18B). The other showed intra-exonal splicing of exon 1 from bp 587 to bb 950 resulting in a 3611 bp splice variant. This variant disrupts the normal ATG start site for translation and results in a new start site at position 1013 in the transcript. This new start site is in frame and would result in a 23 amino acid truncation at the $\mathrm{N}$ terminus of the protein (Figure 18C). It is not clear what the consequences of this truncation are on the function of the Mier3 protein. Additionally, it is not known whether this splice variant is conserved between species.

I attempted to design Taqman gene expression assays to test whether there is a difference in expression of these splice variants between WF and COP mammary gland tissue samples; however, due to the constraints of the Taqman assay design this was not possible. Instead, I resorted to cloning Mier3 amplicons and counting clones (Table 13). Mier3 amplicons were cloned into a pCR2.1-TOPO vector, clones were picked and amplified and run on agarose gels. 96 clones were counted per sample and analyzed as the proportion of full length Mier3 clones out of the total number of clones (containing full length Mier3 plus spliced Mier3) counted. Clone assay results demonstrated there was not a significant difference in Mier3 splice variant expression versus the full length variant expression between WF and resistant WF.COP mammary glands (Figure 18D). Overall, the full length Mier3 transcript expression was much higher than that of the splice variant ( $76 \%$ and $79 \%$ for resistant $\mathrm{N} 3$ and susceptible WF samples, respectively).

Further bioinformatic analysis showed that Mier3 sequence in the mouse and human have an alternatively used exon upstream of exon 1 that results in an alternative start site. The alternate exon usage transcribes three short exons and excludes 
A

C FL SV

B
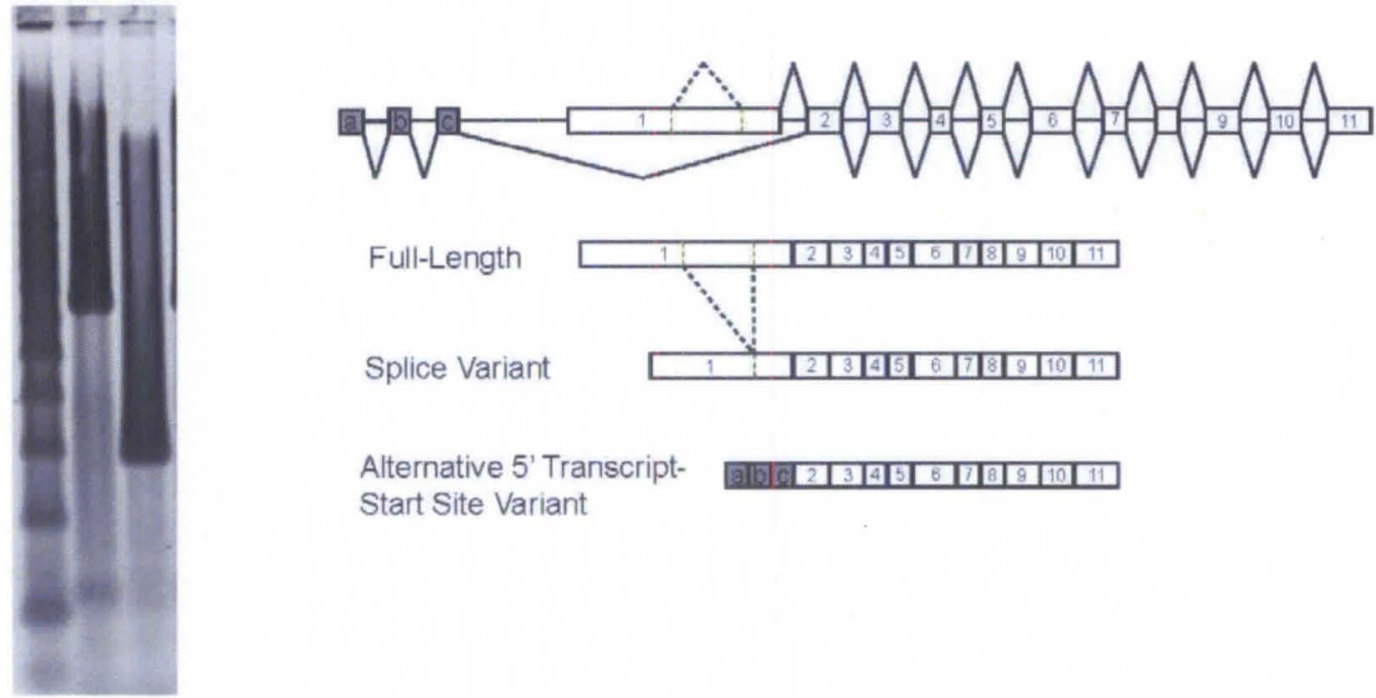

Full-Length

Splice Variant

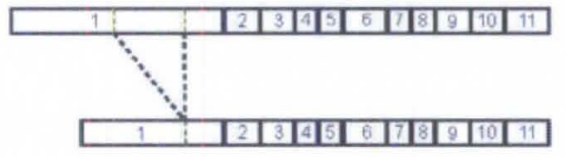

Alternative 5' Transcript-

Start Site Variant

C

Mier3 Full Length

Mier3 Splice Variant

---UTLCFIPVGSLSSEDHDFDPTAEMCVHDYDDERTLEEEELUGDGKNFSSE IEDLER.MW-60.66 kD - MITIGSLSSEDHDFDPTAEMLVHDYDDERTIEEEETMGD GRNFSSE IEDLEK MW-60.41 kD

Mier3 Alternate 5' Start Variant MAEASFGSS SFVGSL SSEDHDFDPTAEMLVHDYDDERTLEEZELMGDGKANFSSE IEDLER MW=61.11 kD

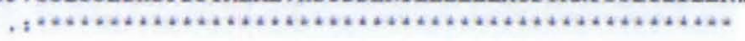

D

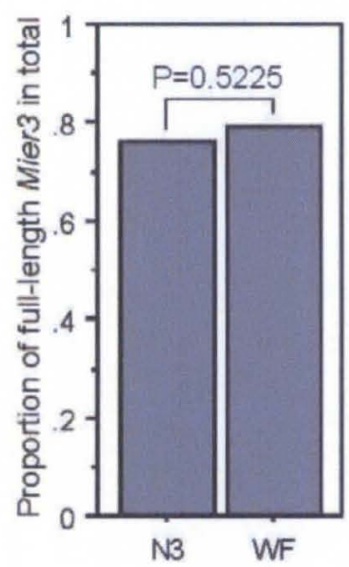

$\mathrm{E}$
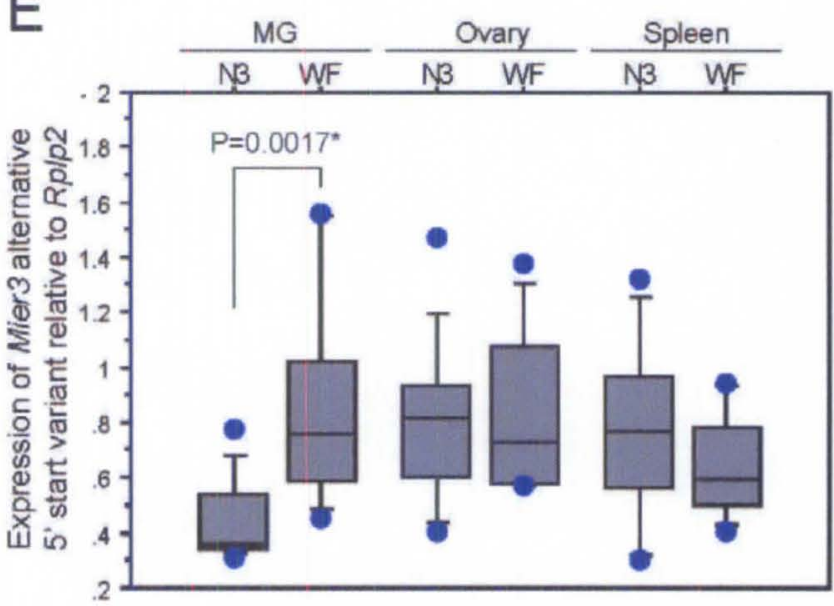
Figure 18: Three different Mier 3 variants are expressed in the rat mammary gland. A; Image of electrophoretic gel of full-length Mier3 and Mier3 splice variant. B; Diagram of gene structure of full-length, spliced, and alternative 5'-transcript start variant of Mier3. C; Amino acid sequence comparison of the three different variants at the Nterminus. Molecular weights of each protein are predicted based on sequence. D; Expression levels of full-length Mier 3 compared to total Mier3 expressed. Levels depicted as proportion of full-length Mier3 in total. P values based on t-test of Arcsinetransformed proportion values. E; Expression levels of alternative 5'-transcription start Mier3 variant. Expression is relative to Rplp2 expression. P values based on results of ANOVA and Fisher PLSD post-hoc test. Abbreviations: C, 100 base pair DNA ladder standard; FL, full length rat Mier3; SV, rat Mier3 splice variant. 


\section{Table 13:}

Primer and Probe Sequences Used for Analysis of Rat Mier3 Variants

\begin{tabular}{|c|c|c|}
\hline Assay Name & Primer/Probe Name & Primer/Probe Sequence \\
\hline \multirow[t]{2}{*}{ Mier3 Splice Variant Cloning and Sequencing } & Mier3-F3 & AGCCCGTGTATTCCAGACAG \\
\hline & Mier3-R3 & TGCAACCGTTGGAATTGTAG \\
\hline \multirow[t]{2}{*}{ Mier3 Alternate 5' Transcription Start Site Sequencing } & Mier3-alt-5'-end-F3 & CTGGCGATTGGCTCAGG \\
\hline & Mier3-alt-5'-end-R3 & TGTCTAGGACTTTTTCATTTCCA \\
\hline \multirow[t]{6}{*}{ Mier3 Alternate 5' Transcription Start Site Taqman Expression } & Mier3-alt5'-1-F & ATGGCGGAGGCTTCCTTT \\
\hline & Mier3-alt5'-1-R & TCAAAATCATGATCCTCAGAAGACA \\
\hline & Mier3-alt5'-1-Probe & AGCCCAGTTGGGTCT \\
\hline & Mier3-alt5'-2-F & AGCCCAGTTGGGTCTTTGTCT \\
\hline & Mier3-alt5'-2-R & TCATAGTCATGGACCAGCACTCA \\
\hline & Mier3-alt5'-2-Probe & CTGAGGATCATGATTTTG \\
\hline
\end{tabular}


exon 1 (Figure 18B). I designed primers to the orthologous rat sequence and amplified and sequenced these in rat mammary glands (Table 13). To further determine whether there were expression differences in the alternative start form of Mier 3 compared to full length Mier3, I designed Taqman assays (Table 13). The results indicate a differential expression of the alternative start end variant between WF and COP untreated mammary glands $(\mathrm{P}=0.0017)$ (Figure 18E). Moreover, differential expression of the alternative 5'start variant appears to be specific to the mammary gland as differential expression was not observed in ovary and spleen tissue (Figure 18E). The alternative start site variant results in a transcription start site beginning upstream of the normal start site; however, the alternate transcription start site stays in frame with the downstream coding of Mier3. Although, the amino acid sequence is slightly altered at the N-terminus of the protein, there is no evidence suggesting this change should disrupt translation (Figure 18C). It is not clear what the implication of this alternative sequence has for Mier 3 function, but expression differences may be implicated in the reduced expression of total Mier 3 seen in previous gene expression assays.

\section{Mcs1b Genotype Exhibits Pleiotropic Effects on Body Weight}

GWA studies have identified several breast cancer susceptibility loci; however, little is known about how the relative risks associated with these regions are affected by the established reproductive, behavioral, and anthropometric risk factors for breast cancer (often referred to collectively as environmental factors, although some, such as height, are in part genetically determined). Travis et al. detected a significant association between human breast cancer risk associated SNP rs889312 and stature in women (Travis 
et al., 2010). To determine if rat Mcs $1 b$ might also exhibit pleiotropy, we analyzed rat body weight, which is information we routinely collect and relevant because body weight is genetically correlated to stature in humans (Czerwinski et al., 2007). Significant effects of $M c s 1 b$ genotype $(\mathrm{P}<0.0001)$ and DMBA exposure $(\mathrm{P}=0.0014)$ on body weight at 12 weeks of age were detected (Figure 19). The interaction between Mcs $1 b$ genotype and DMBA exposure was also significant $(\mathrm{P}=0.0004)$. Females with the $M c s 1 b$ resistant genotype had mean \pm SD body weights of $200 \pm 11$ grams with DMBA $(\mathrm{N}=47)$ and 201 \pm 7.7 grams without $(\mathrm{N}=33)$, which were not significantly different $(\mathrm{P}=0.7880)$. Comparatively, mammary cancer susceptible females had higher $(\mathrm{P}<0.0001)$ mean $\pm \mathrm{SD}$ body weight at $192 \pm 11$ grams with DMBA $(\mathrm{N}=45)$ than unexposed susceptible females $(\mathrm{N}=34)$ who had a mean $\pm \mathrm{SD}$ body weight of $180 \pm 12$ grams.

\section{Rat Mammary Carcinomas Express Higher Mier3 Transcript Levels Compared to Normal Rat Mammary Gland Tissue}

There was a significant difference in expression of Mier3, Gpbp1, and Map3k1 between untreated N3 and WF mammary glands. However, only Mier3 exhibited a significant effect after DMBA induction. Therefore, I next sought to determine whether there was an effect of $M c s 1 b$ genotype on levels of any of these transcripts in mammary carcinoma tissue. Further, Il6st was also included because it had been reported to be higher in rat mammary carcinomas compared to normal mammary gland tissues although in our studies it narrowly missed statistical significance (Qiu et al., 2003b).

Mier3 mRNA expression was measured in DMBA-induced mammary carcinomas from $M c s 1 b$ resistant genotype $(\mathrm{N}=25)$ and susceptible $(\mathrm{N}=28)$ mammary glands by 


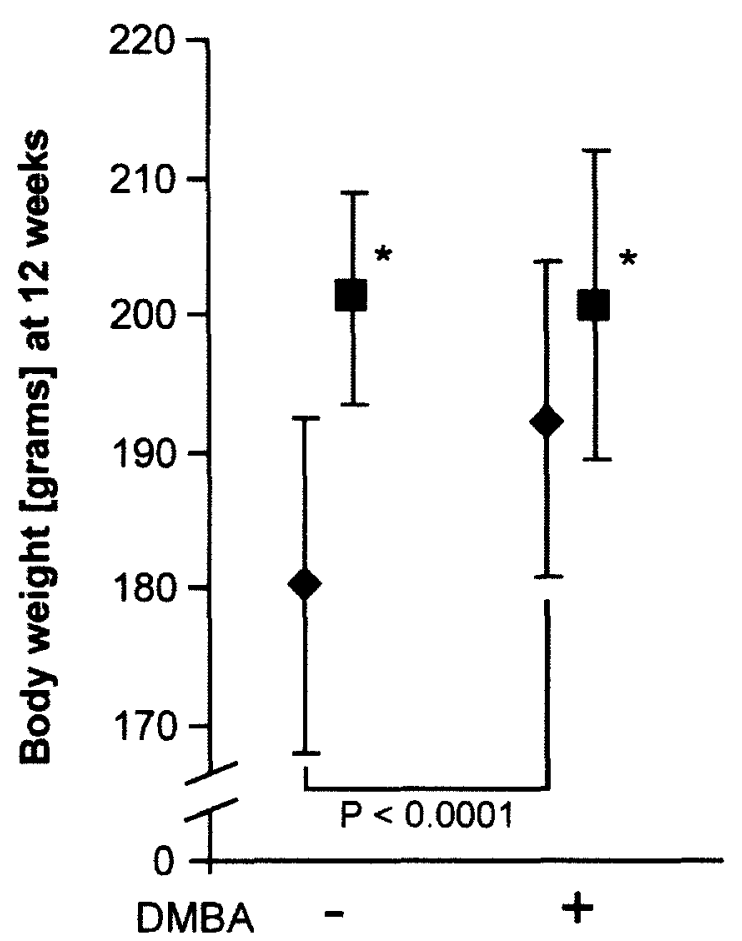

Figure 19: Rat $M c s l b$-resistant genotype is associated with higher body weight. Lower body weight at 12 weeks of age was observed in mammary carcinoma susceptible $(\bullet)$ compared with $M c s l b$-resistant females ( $\square)$ with DMBA and without $(P<0.0001$ and $\mathrm{P}=0.0007$, respectively). Body weight was significantly higher in susceptible females that received DMBA compared to females not receiving DMBA $(P<0.0001)$. 
QPCR and compared to adjacent "non-diseased" mammary glands. We collected total RNA from DMBA-induced mammary carcinomas $(\mathrm{N}=1$ or 2 per rat) and adjacent nondiseased mammary gland tissue from 21-23 week old females $(\mathrm{N}=6$ per genotype). There were no statistically significant differences in mammary carcinoma transcript levels between $M c s 1 b$ genotypes for any of the four genes tested (Figure 20A). However, Mier3 transcript levels were significantly higher $(1.8$-fold $)$ in mammary carcinomas compared to non-diseased mammary tissue. We also observed that Il6st was potentially different between mammary carcinomas and non-diseased mammary glands but did not meet statistical significance (Figure 20A).

To determine whether Mier3 expression differences had been recognized in human samples, I queried The Cancer Genome Atlas (cancergenome.nih.gov) gene expression database by using the Oncomine (Rhodes et al., 2004) data-mining platform. Results show that levels of human MIER3 were, respectively, 1.33 and 1.20 fold higher in invasive ductal $(\mathrm{N}=392)$ and invasive lobular $(\mathrm{N}=36)$ breast carcinoma samples compared to pathologically normal breast tissues $(\mathrm{N}=61)\left(\mathrm{P}=2.8 \times 10^{-13}\right.$, ductal; $\mathrm{P}=6.3 \times 10^{-}$ ${ }^{4}$, lobular; t-tests, Figure 19B). Thus, both human/rat MIER3/Mier3 levels are higher in breast/mammary carcinoma compared to non-diseased breast/mammary tissues.

\section{$\underline{\text { Discussion }}$}

Within the interval delineated by SNP markers $\mathrm{A} 12 \mathrm{v}$ and $\mathrm{A} 12 \mathrm{oo}$ lie thirteen transcripts on rat chromosome 2 (Figure 5). To determine the mechanism by which the $M c s 1 b$ locus confers resistance to developing mammary carcinomas when induced with DMBA I sought to, first, determine if any SNPs in the coding regions of these transcripts 
A

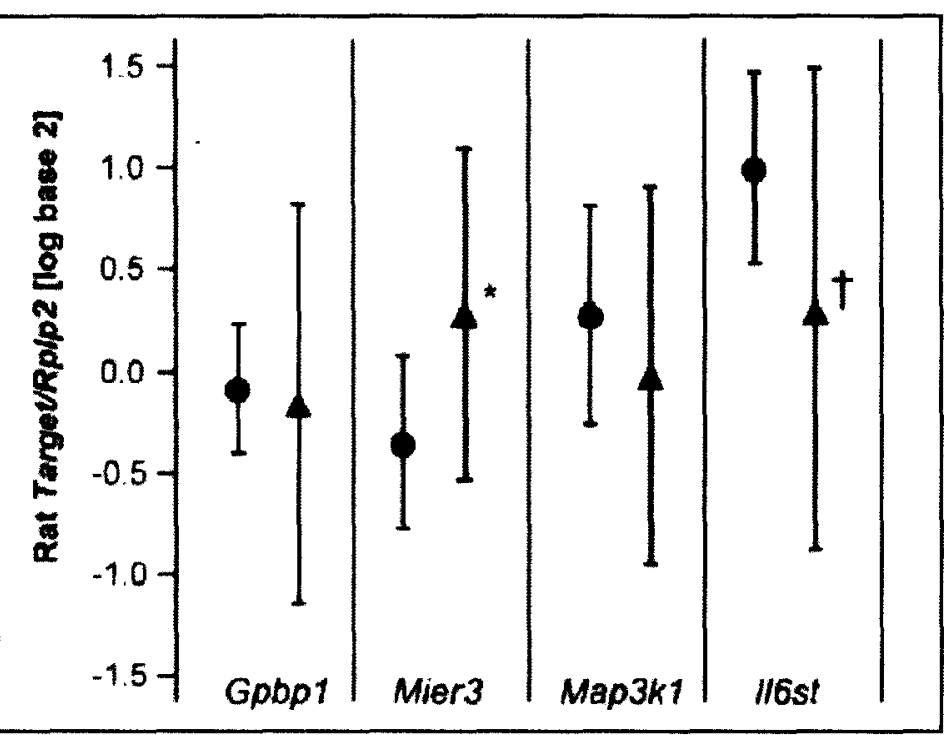

B

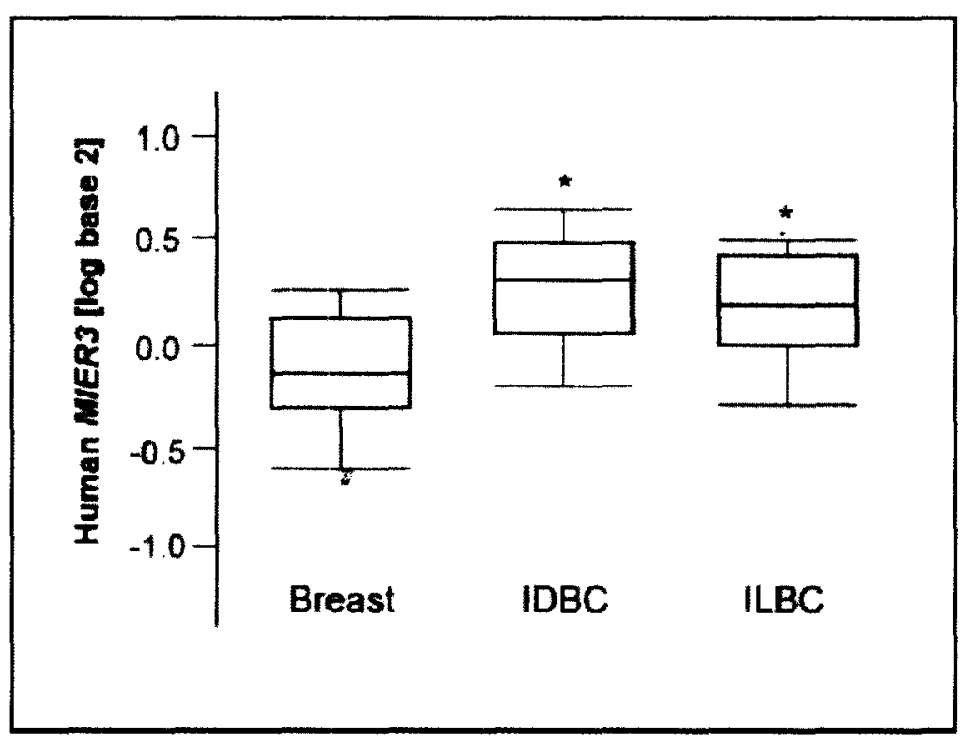


Figure 20: Rat Mier3 transcript levels were significantly higher in DMBA-induced mammary carcinomas compared to non-diseased mammary gland tissue and human MIER3 was significantly higher in breast carcinomas compared with pathologically normal breast tissues. $\mathrm{A}$; Mean $\pm \mathrm{SD}$ are graphed for each variable. Expression of Mier3 mammary carcinoma $(\mathbf{\Delta})$ is significantly different than in adjacent non-diseased mammary gland tissue $(\bullet ; *, \mathrm{P}=0.0120 ; \uparrow, \mathrm{P}=0.0569) . \quad \mathrm{B}$, Oncomine (www.oncomine.org) was used to query The Cancer Genome Atlas (cancergenome.nih.gov) gene expression database. Box plots of $\log _{2}$ median centered MIER3 transcript levels are shown for invasive ductal breast carcinomas (IDBC, $\mathrm{N}=392$ ) and invasive lobular breast carcinomas (ILBC, $\mathrm{N}=36$ ) compared with pathologically normal breast tissues (Breast, $\mathrm{N}=61$ ). MIER3 transcript levels are significantly elevated in both tumor types compared to normal tissue $(*, \mathrm{P}<0.05)$. 
existed between susceptible and resistant lines. Of the thirteen, 9 were amplified and sequenced in rat spleen and thymus tissue: Gpbpl, Map3k1, Mier3, Ankrd55, Il6st, Il31ra, Ddx4, Slc38a9, and Ppap2a. The other four were predicted transcripts and had not been validated. These were identified to be pseudogenes upon further investigation using bioinformatic tools. Sequencing results showed that there was no variation between susceptible WF and resistant WF.COP cDNAs. This suggests that the basis for $M c s 1 b$ COP-conferred resistance is regulatory in nature; therefore, further studies would be needed to identify potential gene candidates.

There are many mechanisms that could be responsible for the $M c s l b$-conferred mammary carcinoma resistance phenotype, e.g., a variant in a promoter or enhancer disturbing transcription or a variant in a splice site disrupting mRNA processing. To investigate this mechanism the most suitable starting point was to examine expression levels of the transcripts. Gene expression of the nine validated transcripts was measured in mammary glands from animals treated with DMBA or not. Three transcripts were differentially expressed between susceptible and resistant untreated mammary gland tissue samples: Gpbpl, Mier3, and Map3k1. However, only Mier3 was statistically different between susceptible and resistant mammary glands after DMBA was administered. Moreover, Il6st was previously reported to be upregulated in mammary carcinomas versus non-diseased mammary gland tissue (Qiu et al., 2003a) but missed statistical significance in my studies. The interaction between $M c s l b$ genotype and DMBA exposure approached statistical significance for Map3kl; however, Map3kI expression was not differentially expressed in DMBA-treated mammary glands. The loss in statistical significance between DMBA-exposed susceptible and Mcs $1 b$ resistant 
females for Map $3 k 1$ was due to an increase in mean level of Map3kl $(\mathrm{P}=0.0003)$ in the $M c s 1 b$ resistant genotype females with DMBA compared to age-matched controls of the same genotype without DMBA (Table 13). Map3kl levels were not different $(\mathrm{P}=0.2038)$ between susceptible WF mammary glands with or without DMBA. Regardless, Map3kl expression was not different between genotypes in DMBA-exposed mammary glands suggesting it was not a likely candidate. Hence, Mier 3 stood out as the most likely candidate. Indeed, further study of Mier3 in rat tumor samples showed that Mier3 expression was also increased in mammary carcinomas compared to adjacent nondiseased mammary gland tissue. This was reinforced by Oncomine microarray data confirming significantly elevated MIER3 expression in human breast cancer samples compared to normal breast tissue.

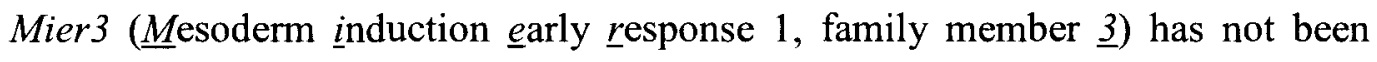
characterized. However, based on sequence similarity, human MIER3 has been identified as having two key domains: ELM2 and SANT (2012). The MIER3 ELM2 domain is from amino acid position 174 to 272 . The ELM2 (Egl-27 and MTA1 homology 2) domain was initially identified in the protein MTA1 a component of the NuRD chromatin regulatory complex and is involved in recruiting HDAC leading to changes in chromatin structure and resulting in transcriptional repression (Ding et al., 2003; Solari et al., 1999). The SANT domain is located from amino acid position 277 to 329. SANT domains are present in nuclear receptor co-repressor proteins and in the subunits of many chromatin-remodeling complexes (Aasland et al., 1996). Moreover, SANT domains are characterized by tandem repeats of three alpha-helices arranged in a helix-turn-helix motif, each alpha helix containing a bulky aromatic residue making them 
similar to the DNA-binding domain of Myb proteins involved in DNA binding and transcriptional repression (Grune et al., 2003; Vargova et al., 2011). Taken together, the presence of these domains suggests that Mier3 may bind DNA or regulate chromatin structure.

Another Mier family member, Mierl, has also been implicated in breast cancer progression (McCarthy et al., 2008a). Human and rat MIER3/Mier3 (GenBank ref) NP_689835.3 and NP_001161472.1) gene products share 93\% amino acid sequence identity, and human MIER3 and MIER1 (GenBank ref|NP_001071172.1) have 54\% identical amino acids based on BLAST (van Boxtel et al., 2011). Mierl is a transcriptional regulator that was discovered during a screen for fibroblast growth factor response genes (Paterno et al., 1997; Paterno et al., 1998; Thorne et al., 2005). Notably, MIER1 physically interacts with ER $\alpha, \mathrm{Sp} 1$, and Creb-binding protein (Blackmore et al., 2008; Ding et al., 2004b; McCarthy et al., 2008b). The impact of hormone receptors and co-regulators on breast cancer development has been noted. Of particular interest, MIER1 contains a C-terminal LxxLL motif referred to as LXD (McCarthy et al., 2008a). The LXD is a highly conserved sequence shown to interact with variety of hormone receptors (Heery et al., 1997). Moreover, studies have shown that different arrangements of the LXD confer varying specificities for different hormone receptors. For example, whereas a single LXD is sufficient for activation by ER $\alpha$, different combinations of two, appropriately spaced, LXDs are required for actions of the thyroid hormone, retinoic acid, peroxisome proliferator-activated, or progesterone receptors (McInerney et al., 1998b). Strikingly, MIER1 contains a single C-terminal LXD while MIER3 contains 2 LXDs. Concordant with this, studies demonstrate that loss of MIERI may contribute to 
breast cancer progression while our data indicate that reduction in Mier3 expression is protective against mammary carcinoma development. Taken together, a potential functional difference between MIERI and MIER3 may be that a difference in the number of LXD motifs between them results in physical interactions with different nuclear hormone receptors.

Amplification of Mier3 in rat thymus cDNA for sequencing resulted in two distinct bands on agarose gel. I further examined these bands by, first, confirming their expression in mammary tissue and sequencing. Sequencing revealed a full-length transcript and a splice variant in which a 363 bp segment was internally spliced out of exon 1. Additionally, the human and mouse MIER3/mier3 annotated sequences indicate that additional exons may exist upstream of exon 1 . To test this, I successfully amplified and sequenced the rat orthologous region. Sequencing identified alternative usage of three short exons upstream of the original exon 1 being transcribed and skipping exon 1 . Further, I measured gene expression of both variants. No differences were identified between the full-length and splice variant forms; however, the alternative start site variant was specifically down-regulated in resistant compared to susceptible mammary gland tissue. This was similar to the gene expression for all Mier3 isoforms performed previously, suggesting that the differences in total Mier3 expression may be defined by expression of the Mier 3 alternative transcription start site variant.

Both Mier3 variants have different translation start sites compared to the original full length Mier3 transcript. This would change the N-terminus of the Mier3 protein for each of these variants; however, neither rat Mier3 nor its human ortholog has been characterized and it is not clear what the consequence of these variants may be on protein 
function. Examining the N-terminus with The Eukaryotic Linear Motif resource (Dinkel et al., 2012) (http://elm.eu.org) shows that full length Mier3 contains a USP7-binding motif that is lost in the intra-exonal splice variant. Additionally, the alternative transcription start variant retains this USP7-binding domain and adds a Casein kinase 1 (CK1) phosphorylation site (Dinkel et al., 2012). USP7 is a deubiquitinating enzyme most commonly involved in regulation p53 regulation by deubiquitinating it and protecting it from MDM2-mediated degradation ( $\mathrm{Li}$ et al., 2002). CK1 is a serine/threonine kinase involved in a variety of cell signaling pathways with a myriad of targets; however, it has been recognized to be involved in the activation of the Wnt signaling pathway (Davidson et al., 2005). Therefore, the alternative start site form of Mier3 may be more mitogenic compared to the other isotypes. Taking everything together, Mier3 emerges as a positive regulator of cell proliferation affecting carcinogenesis in the mammary gland.

Travis et al. reported pleiotropic effects on stature in women associated with the breast cancer-associated SNP rs889312 (Travis et al., 2010). During the course of our experiments we routinely gather data on body mass of the rats. I used this to test whether a correlation existed between body mass and $M c s 1 b$ genotype since body mass can be used as a proxy for stature in humans. Lower body weight was observed in susceptible female rats compared to resistant congenic rats at 12 weeks of age. These animals differ essentially only at the Mcs $1 b$ locus and resistant female rats express less Mier 3 than female rats harboring a susceptible Mcs $1 \mathrm{~b}$ allele. Therefore, this seems counter-intuitive as it is believed that Mier3 is mitogenic and increased expression would result in increased weight gain. However, as previously mentioned, PR is known to attenuate 
effects of ER $\alpha$ (Hsueh et al., 1976; Tseng and Gurpide, 1975). What is more, loss of estrogen activity has been identified to associate with a reduction in catabolism and an increase in weight, femur length, and bone density in male mice (Ford et al., 2011). As discussed earlier in this chapter, human MIER3 contains 2 LXDs which may confer affinity for PR (McInerney et al., 1998b). Therefore, a likely scenario would be that in resistant congenic females expressing lower amounts of Mier3, there is less PR bound to Mier 3 and more that is available to inhibit ER $\alpha$ activity. ER $\alpha$ inhibition in these animals results in higher body mass compared to susceptible animals expressing higher amounts of Mier3. However, this is based on an assumption that Mier3 is binding to PR supported only by sequence similarity to Mierl and the presence of 2 LxxLL motifs. Therefore, this is entirely speculative and more empirical work is necessary to determine the mechanism by which Mier3 is working. 


\section{CHAPTER V \\ CONCLUDING REMARKS}

Rat mammary carcinoma susceptibility, like human breast cancer risk, is complex as both are controlled by multiple susceptibility alleles and environmental factors. We have mapped rat $M c s l b$ to a $1.1 \mathrm{Mb}$ region of rat chromosome 2 using multiple congenic lines. We found that rat $M c s 1 b$ is highly relevant to human breast cancer susceptibility as it contains genomic sequence orthologous to a low-penetrance breast cancer risk allele at human chromosome 5q11.2. This human susceptibility allele was first reported by Easton et al.(2007) in the first population-based breast cancer risk GWAS. Human $5 q 11.2$ has been confirmed to strongly associate with breast cancer risk in multiple independent studies of European- and Asian-descent populations (Antoniou et al., 2008; Broeks et al., 2011; Campa et al., 2011; Garcia-Closas et al., 2008a; Han et al., 2011; Zheng et al., 2010). This is the first report of a rodent complex disease susceptibility QTL with a GWAS-identified concordant human ortholog that had a probability of association below a stringent significance level of $P \leq 10^{-7}$, which is widely deemed to be required for genome-wide studies.

An experimental organism with a segregating concordant susceptibility allele implies that functional genetic studies may translate directly to human biology and disease. For example, Gould and colleagues reported that rat Mcs5a, a WKY strain resistance QTL that is concordant to human $M C S 5 A$, acted in a non-mammary cell- 
autonomous manner that involves immune cells (Smits et al., 2011a). Here, we used rat genetic lines to show that $M c s l b$ controls mammary cancer susceptibility by an undetermined mammary gland cell autonomous mechanism. While our result is in agreement with previous work that concluded a majority, but not all, of the COP rat strain resistance to mammary cancer is mammary gland autonomous (Zhang et al., 1990); it further highlights that the WKY and COP strains may achieve mammary carcinoma resistance through different genetically determined cellular and molecular mechanisms that are likely genetically determined in humans as well.

Further, Gould and colleagues developed a clonogenic transplant assay using rat MEC preparations to reconstitute mammary glands in the intrascapular fat pads of recipient rats (Gould and Clifton, 1985). To date no one has demonstrated an ability to induce carcinomas in these ectopic glands. The window for DMBA to induce carcinogenesis in endogenous rat mammary glands and whole mammary gland transplants was established to be at 50-55 days of age. Herein, I show that glands formed from the injection of MECs have a longer latency ${ }^{-}$compared to whole mammary gland grafts since DMBA-induced carcinogenesis is delayed to $70-75$ days of age. This is a significant result for future use of this protocol.

Most common genetic variation associated with human complex disease susceptibility appears to be located in non-protein-coding DNA. Since we found no genetic variation between susceptible and resistant allele $M c s 1 b$ ORFs, we conclude that $M c s 1 b$ is likely a noncoding gene regulatory element(s), such as a transcription factor binding site or noncoding RNA. This would be similar to the hypothesized identity of the human $5 q 11.2$ breast cancer risk associated element. Human polymorphisms that are 
contained in public databases and highly correlated with human $5 q 11.2$ breast cancer risk associated SNP rs889312 are in non-protein-coding DNA. There are no known noncoding RNAs in either the human or rat ortholog; therefore, another type of gene regulatory element is likely responsible for or associated to susceptibility differences.

Our studies suggest that MIER3 is a strong candidate breast cancer susceptibility gene at human $5 q 11.2$. We identified Mier3 as a strong Mcs $1 b$ candidate gene in this study based on different Mier3 mammary gland transcript levels between susceptible and $M c s 1 b$ resistant genotypes. Lower levels of Mier3 in Mcslb resistant genotype females were genetically determined and not dependent on the induction of mammary carcinogenesis by DMBA. We also found Mier3 levels significantly lower in nondiseased rat mammary tissue compared to mammary carcinoma. Further, we queried The Cancer Genome Atlas gene expression database and noted that human MIER3 levels were higher in both ductal and lobular breast carcinomas compared to breast tissue.

MIER3 or mesoderm induction early response 1, family member 3 (GenBank ref(NM_152622) is an uncharacterized gene. We determined that MIER3 localized to the nucleus. Human and rat MIER3/Mier3 (GenBank ref] NP_689835.3 and NP_001161472.1) gene products share 93\% amino acid sequence identity, and human MIER3 and MIER1 (GenBank ref|NP_001071172.1) have 54\% identical amino acids based on BLAST (van Boxtel et al., 2011). MIER1 physically interacts with ER $\alpha, \mathrm{Sp} 1$, and Creb-binding protein (Blackmore et al., 2008; Ding et al., 2004b; McCarthy et al., 2008b). MIER1 contains one, while MIER3 has two conserved LxxLL sequences, which is a motif that facilitates nuclear hormone receptor interactions (Heery et al., 1997). A potential functional difference between MIER1 and MIER3 may be that a difference in 
the number of LxxLL motifs between them results in physical interactions with different nuclear hormone receptors (McInerney et al., 1998a). In addition, MIER1 and MIER3 harbor ELM2 and SANT domains. The ELM2 domain is involved in recruitment of HDAC activity, which leads to changes in chromatin structure and results in transcriptional repression (Ding et al., 2003). Likewise, the MIER1 SANT domain functions in gene repression by interacting with $\mathrm{Sp} 1$ and interfering with its ability to bind to its cognate site on responsive promoters (Ding et al., 2004a). The presence of two LxxLL motifs along with the ELM2 and SANT domains suggests that MIER3 is a potential transcriptional repressor whose activity is mediated by interaction with nuclear hormone receptors. To test this idea, more functional studies are necessary to determine the mechanism by which Mier3 is controlling mammary carcinogenesis in the rat.

In addition to MIER3, MAP3KI and C5ORF35 reside within the human $5 q 11.2$ haplotype block that associates with breast cancer risk. Even though there are no published studies in support, $M A P 3 K 1$ is often considered the candidate breast cancer susceptibility gene at $5 q 11.2$ due to its location within the breast cancer risk associated haplotype block and known function as a serine/threonine kinase. In our rat studies, Map3kl was differentially expressed between susceptible and $M c s l b$ resistant congenic rats that had not been induced to undergo mammary carcinogenesis; however, mammary glands that had been exposed to mammary carcinogen did not show a difference in Map3k1 levels between $M c l b$ alleles. An interesting result in our study with respect to Map3kl, which may have important implications for human studies of potential genotype-environment interactions, is exposure to mammary carcinogen resulted in increased mammary gland Map3kI levels for the $M c s I b$ resistant, but not the susceptible 
genotype. We found no evidence of a rat orthologous transcript to human C5ORF35 in multiple rat tissues. Further, exonic elements of C5ORF35 have not been conserved in the rat. Therefore, we conclude that MAP3KI and C5ORF35 are not as likely as MIER3 to be breast cancer susceptibility genes.

I also identified multiple variants of Mier 3 expressed in the rat mammary gland. One of these variants exhibits alternate exon usage compared to the annotated Mier3 transcript (GenBank ref|NM_152622). Similar to what was observed in the original Mier 3 gene expression studies, expression of the alternate exon variant is reduced in resistant Mcslb mammary gland tissue compared to susceptible controls. The sequence of the variant differs only at the 5 ' end of the transcript, but is predicted to introduce a casein kinase phosphorylation site at the N-terminus. However, the implications are not clear and more study is necessary to determine whether differential expression of this variant affects mammary carcinoma susceptibility. Regardless, differential expression of this variant adds to our evidence that Mier 3 is the most likely breast cancer gene.

We noted that both rat Mcs $1 b$ and human $5 q 11.2$ exhibit pleiotropy. Travis et al. reported that carriers of the increased risk allele at human $5 q 11.2$ were significantly shorter in height than non-carriers (Travis et al., 2010). In our study, high risk female rats had lower body weight than $M c s l b$ resistant females. There is a predicted rat body weight QTL named $B w l$ that overlaps $M c s 1 b$ and is associated with mesenteric body fat amount (Ogino et al., 2000). Both human and rat study results are counter intuitive as one might expect taller women and heavier rats to be at greater cancer risk. Thus, it is important to note that, as expected with low-penetrance alleles, the quantitative difference between the means for each human genotype were subtle with overlapping 
distributions. Mean height difference was $7 \mathrm{~mm}$ between non-carriers and carriers of the increased risk allele. In our study, we analyzed only body weight, and not specific components of body weight, such as bone density or fat. Thus, better descriptive traits would likely be more informative. It is notable that the pleitropic effects of these alleles opens the possibility that other experimental organisms, approaches, and study designs without focus on breast or mammary cancer may be useful to functionally characterize breast cancer risk associated genetic variation at 5 q11.2.

In conclusion, rat $M c s I b$ contains a mammary gland-autonomous allele and a non-protein-coding genetic element that is orthologous to the GWAS-identified human $5 q 11.2$ breast cancer susceptibility locus. We propose that MIER 3 is a strong candidate breast cancer susceptibility gene. 


\section{REFERENCES}

(2005). A haplotype map of the human genome. Nature 437, 1299-320.

(2012). Reorganizing the protein space at the Universal Protein Resource (UniProt). Nucleic Acids Res 40, D71-5.

Aasland, R., Stewart, A. F. and Gibson, T. (1996). The SANT domain: a putative DNA-binding domain in the SWI-SNF and ADA complexes, the transcriptional corepressor N-CoR and TFIIIB. Trends Biochem Sci 21, 87-8.

Ahmed, S. Thomas, G. Ghoussaini, M. Healey, C. S. Humphreys, M. K. Platte, R. Morrison, J. Maranian, M. Pooley, K. A. Luben, R. et al. (2009). Newly discovered breast cancer susceptibility loci on 3p24 and 17q23.2. Nat Genet 41, 585-90.

Aitman, T. J., Critser, J. K., Cuppen, E., Dominiczak, A., Fernandez-Suarez, X. M., Flint, J., Gauguier, D., Geurts, A. M., Gould, M., Harris, P. C. et al. (2008). Progress and prospects in rat genetics: a community view. Nat Genet 40, 516-22.

Altekruse SF, K. C., Krapcho M, Neyman N, Aminou R, Waldron W, Ruhl J, Howlader N, Tatalovich Z, Cho H, Mariotto A, Eisner MP, Lewis DR, Cronin K, Chen HS, Feuer EJ, Stinchcomb DG, Edwards BK (eds). (2010). SEER Cancer Statistics Review, 1975-2007. In National Cancer Institute., (ed. Bethesda, MD.

Altshuler, D., Daly, M. J. and Lander, E. S. (2008). Genetic mapping in human disease. Science (New York, N Y) 322, 881-8.

American Cancer Society. (2011). Breast Cancer Facts \& Figures 2011-2012, (ed. Atlanta: American Cancer Society.

American Cancer Society. (2012). Cancer Facts and Figures 2012, (ed. Atlanta: American Cancer Society.

Anderson, G. L., Chlebowski, R. T., Aragaki, A. K., Kuller, L. H., Manson, J. E., Gass, M., Bluhm, E., Connelly, S., Hubbell, F. A., Lane, D. et al. (2012). Conjugated equine oestrogen and breast cancer incidence and mortality in postmenopausal women with hysterectomy: extended follow-up of the Women's Health Initiative randomised placebo-controlled trial. Lancet Oncol 13, 476-86.

Anderson, W. F. and Devesa, S. S. (2005). Breast carcinoma in men. Cancer 103, 4323; author reply 433. 
Antoniou, A., Pharoah, P. D., Narod, S., Risch, H. A., Eyfjord, J. E., Hopper, J. L., Loman, N., Olsson, H., Johannsson, O., Borg, A. et al. (2003). Average risks of breast and ovarian cancer associated with BRCA1 or BRCA2 mutations detected in case Series unselected for family history: a combined analysis of 22 studies. Am J Hum Genet 72 , 1117-30.

Antoniou, A. C., Spurdle, A. B., Sinilnikova, O. M., Healey, S., Pooley, K. A., Schmutzler, R. K., Versmold, B., Engel, C., Meindl, A., Arnold, N. et al. (2008). Common breast cancer-predisposition alleles are associated with breast cancer risk in BRCA1 and BRCA2 mutation carriers. Am J Hum Genet 82, 937-48.

Antoniou, A. C. Wang, X. Fredericksen, Z. S. McGuffog, L. Tarrell, R. Sinilnikova, O. M. Healey, S. Morrison, J. Kartsonaki, C. Lesnick, T. et al. (2010). A locus on 19 p13 modifies risk of breast cancer in BRCA1 mutation carriers and is associated with hormone receptor-negative breast cancer in the general population. Nat Genet 42, 885-92.

Badano, J. L. and Katsanis, N. (2002). Beyond Mendel: an evolving view of human genetic disease transmission. Nat Rev Genet 3, 779-89.

Bahia, H., Ashman, J. N., Cawkwell, L., Lind, M., Monson, J. R., Drew, P. J. and Greenman, J. (2002). Karyotypic variation between independently cultured strains of the cell line MCF-7 identified by multicolour fluorescence in situ hybridization. Int J Oncol 20, 489-94.

Barrett, J. C. and Cardon, L. R. (2006). Evaluating coverage of genome-wide association studies. Nat Genet 38, 659-62.

Barrett, J. C., Fry, B., Maller, J. and Daly, M. J. (2005). Haploview: analysis and visualization of LD and haplotype maps. Bioinformatics 21, 263-5.

Bartella, V., Rizza, P., Barone, I., Zito, D., Giordano, F., Giordano, C., Catalano, S., Mauro, L., Sisci, D., Panno, M. L. et al. (2012). Estrogen receptor beta binds Spl and recruits a corepressor complex to the estrogen receptor alpha gene promoter. Breast Cancer Res Treat.

Beatson, G. T. (1896). On the treatment of inoperable cases of carcinoma of the mamma : suggestions for a new method of treatment, with illustrative cases. Glasgow: Printed by Alex. Macdougall.

Beral, V. (2003). Breast cancer and hormone-replacement therapy in the Million Women Study. Lancet 362, 419-27.

Bevier, M., Sundquist, K. and Hemminki, K. (2011). Risk of breast cancer in families of multiple affected women and men. Breast Cancer Res Treat.

Blackmore, T., Mercer, C., Paterno, G. and Gillespie, L. (2008). The transcriptional cofactor MIER 1-beta negatively regulates histone acetyltransferase activity of the CREBbinding protein. BMC Research Notes 1, 68. 
Boice, J. D., Jr., Bigbee, W. L., Mumma, M. T. and Blot, W. J. (2003). Cancer incidence in municipalities near two former nuclear materials processing facilities in Pennsylvania. Health Phys 85, 678-90.

Bonde, J. P., Hansen, J., Kolstad, H. A., Mikkelsen, S., Olsen, J. H., Blask, D. E., Harma, M., Kjuus, H., de Koning, H. J., Olsen, J. et al. (2012). Work at night and breast cancer - report on evidence-based options for preventive actions. Scand J Work Environ Health.

Boonyaratanakornkit, V., Scott, M. P., Ribon, V., Sherman, L., Anderson, S. M., Maller, J. L., Miller, W. T. and Edwards, D. P. (2001). Progesterone receptor contains a proline-rich motif that directly interacts with SH3 domains and activates c-Src family tyrosine kinases. Mol Cell 8, 269-80.

Boyd, N. F., Connelly, P., Byng, J., Yaffe, M., Draper, H., Little, L., Jones, D., Martin, L. J., Lockwood, G. and Tritchler, D. (1995). Plasma lipids, lipoproteins, and mammographic densities. Cancer Epidemiol Biomarkers Prev 4, 727-33.

Broeks, A. Schmidt, M. K. Sherman, M. E. Couch, F. J. Hopper, J. L. Dite, G. S. Apicella, C. Smith, L. D. Hammet, F. Southey, M. C. et al. (2011). Low penetrance breast cancer susceptibility loci are associated with specific breast tumor subtypes: findings from the Breast Cancer Association Consortium. Human Molecular Genetics 20, 3289-303.

Bulun, S. E., Chen, D., Moy, I., Brooks, D. C. and Zhao, H. (2012). Aromatase, breast cancer and obesity: a complex interaction. Trends Endocrinol Metab 23, 83-9.

Burdall, S. E., Hanby, A. M., Lansdown, M. R. and Speirs, V. (2003). Breast cancer cell lines: friend or foe? Breast Cancer Res 5, 89-95.

Campa, D., Kaaks, R., Le Marchand, L., Haiman, C. A., Travis, R. C., Berg, C. D., Buring, J. E., Chanock, S. J., Diver, W. R., Dostal, L. et al. (2011). Interactions between genetic variants and breast cancer risk factors in the breast and prostate cancer cohort consortium. Journal of the National Cancer Institute 103, 1252-63.

Campeau, P. M., Foulkes, W. D. and Tischkowitz, M. D. (2008). Hereditary breast cancer: new genetic developments, new therapeutic avenues. Hum Genet 124, 31-42.

Cardiff, R. D. and Kenney, N. (2007). Mouse mammary tumor biology: a short history. Adv Cancer Res 98, 53-116.

Carmichael, A. R. (2006). Obesity and prognosis of breast cancer. Obes Rev 7, 333-40.

Casey, M. L., MacDonald, P. C. and Andersson, S. (1994). 17 beta-Hydroxysteroid dehydrogenase type 2: chromosomal assignment and progestin regulation of gene expression in human endometrium. $J$ Clin Invest 94, 2135-41.

Cavalieri, E. L., Rogan, E. G. and Chakravarti, D. (2002). Initiation of cancer and other diseases by catechol ortho-quinones: a unifying mechanism. Cell Mol Life Sci 59, 665-81. 
Chlebowski, R. T., Anderson, G. L., Gass, M., Lane, D. S., Aragaki, A. K., Kuller, L. H., Manson, J. E., Stefanick, M. L., Ockene, J., Sarto, G. E. et al. (2010). Estrogen plus progestin and breast cancer incidence and mortality in postmenopausal women. $J A M A$ : the journal of the American Medical Association 304, 1684-92.

Chlebowski, R. T., Hendrix, S. L., Langer, R. D., Stefanick, M. L., Gass, M., Lane, D., Rodabough, R. J., Gilligan, M. A., Cyr, M. G., Thomson, C. A. et al. (2003). Influence of estrogen plus progestin on breast cancer and mammography in healthy postmenopausal women: the Women's Health Initiative Randomized Trial. JAMA : the journal of the American Medical Association 289, 3243-53.

Christou, M., Moore, C. J., Gould, M. N. and Jefcoate, C. R. (1987). Induction of mammary cytochromes P-450: an essential first step in the metabolism of 7,12dimethylbenz[a]anthracene by rat mammary epithelial cells. Carcinogenesis 8, 73-80.

Christou, M., Savas, U., Schroeder, S., Shen, X., Thompson, T., Gould, M. N. and Jefcoate, C. R. (1995). Cytochromes CYP1A1 and CYP1B1 in the rat mammary gland: cell-specific expression and regulation by polycyclic aromatic hydrocarbons and hormones. Mol Cell Endocrinol 115, 41-50.

Collaborative Group on Hormonal Factors in Breast Cancer. (2001). Familial breast cancer: collaborative reanalysis of individual data from 52 epidemiological studies including 58,209 women with breast cancer and 101,986 women without the disease. Lancet 358, 1389-99.

Costantino, J. P., Gail, M. H., Pee, D., Anderson, S., Redmond, C. K., Benichou, J. and Wieand, H. S. (1999). Validation studies for models projecting the risk of invasive and total breast cancer incidence. J Natl Cancer Inst 91, 1541-8.

Cotroneo, M. S., Merry, G. M., Haag, J. D., Lan, H., Shepel, L. A. and Gould, M. N. (2006). The Mcs7 quantitative trait locus is associated with an increased susceptibility to mammary cancer in congenic rats and an allele-specific imbalance. Oncogene 25, 5011-7.

Coussens, L., Yang-Feng, T. L., Liao, Y. C., Chen, E., Gray, A., McGrath, J., Seeburg, P. H., Libermann, T. A., Schlessinger, J., Francke, U. et al. (1985). Tyrosine kinase receptor with extensive homology to EGF receptor shares chromosomal location with neu oncogene. Science (New York, NY) 230, 1132-9.

Cox, A., Dunning, A. M., Garcia-Closas, M., Balasubramanian, S., Reed, M. W., Pooley, K. A., Scollen, S., Baynes, C., Ponder, B. A., Chanock, S. et al. (2007). A common coding variant in CASP8 is associated with breast cancer risk. Nat Genet 39, 352-8.

Cui, J., Germer, K., Wu, T., Wang, J., Luo, J., Wang, S. C., Wang, Q. and Zhang, X. (2012). Cross-talk between HER2 and MED1 regulates tamoxifen resistance of human breast cancer cells. Cancer Res 72, 5625-34.

Czerwinski, S. A., Lee, M., Choh, A. C., Wurzbacher, K., Demerath, E. W., Towne, B. and Siervogel, R. M. (2007). Genetic factors in physical growth and development and 
their relationship to subsequent health outcomes. American journal of human biology : the official journal of the Human Biology Council 19, 684-91.

Darbre, P. D. and Charles, A. K. (2010). Environmental oestrogens and breast cancer: evidence for combined involvement of dietary, household and cosmetic xenoestrogens. Anticancer Res 30, 815-27.

Davidson, G., Wu, W., Shen, J., Bilic, J., Fenger, U., Stannek, P., Glinka, A. and Niehrs, C. (2005). Casein kinase 1 gamma couples Wnt receptor activation to cytoplasmic signal transduction. Nature 438, 867-72.

de Jong, M. M., Nolte, I. M., te Meerman, G. J., van der Graaf, W. T., Oosterwijk, J. C., Kleibeuker, J. H., Schaapveld, M. and de Vries, E. G. (2002). Genes other than BRCA1 and BRCA2 involved in breast cancer susceptibility. J Med Genet 39, 225-42.

de Lorimier, A. A. (2000). Alcohol, wine, and health. Am J Surg 180, 357-61.

De Stefani, E., Ronco, A., Mendilaharsu, M., Guidobono, M. and Deneo-Pellegrini, H. (1997). Meat intake, heterocyclic amines, and risk of breast cancer: a case-control study in Uruguay. Cancer Epidemiol Biomarkers Prev 6, 573-81.

Deitz, A. C., Zheng, W., Leff, M. A., Gross, M., Wen, W. Q., Doll, M. A., Xiao, G. H., Folsom, A. R. and Hein, D. W. (2000). N-Acetyltransferase-2 genetic polymorphism, well-done meat intake, and breast cancer risk among postmenopausal women. Cancer Epidemiol Biomarkers Prev 9, 905-10.

den Dekker, A. D., Xu, X., Vaughn, M. D., Puckett, A. H., Gardner, L. L., Lambring, C. J., Deschenes, L. and Samuelson, D. J. (2012). Rat Mcs $1 b$ is concordant to the human GWAS-identified breast cancer risk locus at $5 q 11.2$ and Mier 3 is a candidate susceptibility gene Submitted.

Ding, Z., Gillespie, L. L., Mercer, F. C. and Paterno, G. D. (2004a). The SANT domain of human MI-ER1 interacts with $\mathrm{Spl}$ to interfere with GC box recognition and repress transcription from its own promoter. $J$ Biol Chem 279, 28009-16.

Ding, Z., Gillespie, L. L., Mercer, F. C. and Paterno, G. D. (2004b). The SANT Domain of Human MI-ER1 Interacts with Sp1 to Interfere with GC Box Recognition and Repress Transcription from Its Own Promoter. Journal of Biological Chemistry 279, 28009-28016.

Ding, Z., Gillespie, L. L. and Paterno, G. D. (2003). Human MI-ER1 alpha and beta function as transcriptional repressors by recruitment of histone deacetylase 1 to their conserved ELM2 domain. Mol Cell Biol 23, 250-8.

Dinkel, H., Michael, S., Weatheritt, R. J., Davey, N. E., Van Roey, K., Altenberg, B., Toedt, G., Uyar, B., Seiler, M., Budd, A. et al. (2012). ELM--the database of eukaryotic linear motifs. Nucleic Acids Res 40, D242-51.

Dobrzycka, K. M., Townson, S. M., Jiang, S. and Oesterreich, S. (2003). Estrogen receptor corepressors -- a role in human breast cancer? Endocr Relat Cancer 10, 517-36. 
Dunning, W. F. and Curtis, M. R. (1952). The incidence of diethylstilbestrol-induced cancer in reciprocal $\mathrm{F}$ hybrids obtained from crosses between rats of inbred lines that are susceptible and resistant to the induction of mammary cancer by this agent. Cancer Res 12, 702-6.

Dunning, W. F., Curtis, M. R. and Segaloff, A. (1947). Strain Differences in Response to Diethylstilbestrol and the Induction of Mammary Gland and Bladder Cancer in the Rat. Cancer Res 7, 511-521.

Dunning, W. F., Curtis, M. R. and Segaloff, A. (1953). Strain differences in response to estrone and the induction of mammary gland, adrenal, and bladder cancer in rats.

Cancer Res 13, 147-52.

Dutertre, M. and Smith, C. L. (2000). Molecular mechanisms of selective estrogen receptor modulator (SERM) action. The Journal of pharmacology and experimental therapeutics 295, 431-7.

Dwinell, M. R., Worthey, E. A., Shimoyama, M., Bakir-Gungor, B., DePons, J., Laulederkind, S., Lowry, T., Nigram, R., Petri, V., Smith, J. et al. (2009). The Rat Genome Database 2009: variation, ontologies and pathways. Nucleic Acids Res 37, D744-9.

Easton, D. F. (1999). How many more breast cancer predisposition genes are there? Breast Cancer Res 1, 14-7.

Easton, D. F. Pooley, K. A. Dunning, A. M. Pharoah, P. D. Thompson, D. Ballinger, D. G. Struewing, J. P. Morrison, J. Field, H. Luben, R. et al. (2007). Genome-wide association study identifies novel breast cancer susceptibility loci. Nature 447, 1087-93.

Erkko, H., Xia, B., Nikkila, J., Schleutker, J., Syrjakoski, K., Mannermaa, A., Kallioniemi, A., Pylkas, K., Karppinen, S. M., Rapakko; K. et al. (2007). A recurrent mutation in PALB2 in Finnish cancer families. Nature 446, 316-9.

Euhus, D. M., Leitch, A. M., Huth, J. F. and Peters, G. N. (2002). Limitations of the Gail model in the specialized breast cancer risk assessment clinic. Breast $J$ 8, 23-7.

Evans, D. G. and Howell, A. (2007). Breast cancer risk-assessment models. Breast Cancer Res 9, 213.

Falany, J. L. and Falany, C. N. (1996). Regulation of estrogen sulfotransferase in human endometrial adenocarcinoma cells by progesterone. Endocrinology 137, 1395 401 .

Fernandez, S. V. and Russo, J. (2010). Estrogen and xenoestrogens in breast cancer. Toxicol Pathol 38, 110-22.

Fisher, B., Costantino, J. P., Redmond, C. K., Fisher, E. R., Wickerham, D. L. and Cronin, W. M. (1994). Endometrial cancer in tamoxifen-treated breast cancer patients: findings from the National Surgical Adjuvant Breast and Bowel Project (NSABP) B-14. $J$ Natl Cancer Inst 86, 527-37. 
Flemming, J., Madarnas, Y. and Franek, J. A. (2009). Fulvestrant for systemic therapy of locally advanced or metastatic breast cancer in postmenopausal women: a systematic review. Breast Cancer Res Treat 115, 255-68.

Fletcher, O. and Houlston, R. S. (2010). Architecture of inherited susceptibility to common cancer. Nat Rev Cancer 10, 353-61.

Flicek, P., Amode, M. R., Barrell, D., Beal, K., Brent, S., Chen, Y., Clapham, P., Coates, G., Fairley, S., Fitzgerald, S. et al. (2011). Ensembl 2011. Nucleic Acids Research 39, D800-D806.

Ford, D., Easton, D. F. and Peto, J. (1995). Estimates of the gene frequency of BRCA1 and its contribution to breast and ovarian cancer incidence. Am J Hum Genet 57, 145762 .

Ford, D., Easton, D. F., Stratton, M., Narod, S., Goldgar, D., Devilee, P., Bishop, D. T., Weber, B., Lenoir, G., Chang-Claude, J. et al. (1998). Genetic heterogeneity and penetrance analysis of the BRCA1 and BRCA2 genes in breast cancer families. The Breast Cancer Linkage Consortium. Am J Hum Genet 62, 676-89.

Ford, J., Hajibeigi, A., Long, M., Hahner, L., Gore, C., Hsieh, J. T., Clegg, D., Zerwekh, J. and Oz, O. K. (2011). GPR30 deficiency causes increased bone mass, mineralization, and growth plate proliferative activity in male mice. $J$ Bone Miner Res $\mathbf{2 6}$, 298-307.

Foulkes, W. D., Ghadirian, P., Akbari, M. R., Hamel, N., Giroux, S., Sabbaghian, N., Darnel, A., Royer, R., Poll, A., Fafard, E. et al. (2007). Identification of a novel truncating PALB2 mutation and analysis of its contribution to early-onset breast cancer in French-Canadian women. Breast Cancer Res 9, R83.

Frazer, K. A., Murray, S. S., Schork, N. J. and Topol, E. J. (2009). Human genetic variation and its contribution to complex traits. Nat Rev Genet 10, 241-51.

Gail, M. H., Brinton, L. A., Byar, D. P., Corle, D. K., Green, S. B., Schairer, C. and Mulvihill, J. J. (1989). Projecting individualized probabilities of developing breast cancer for white females who are being examined annually. $J$ Natl Cancer Inst 81, 187986.

Gao, J., Lauer, F. T., Dunaway, S. and Burchiel, S. W. (2005). Cytochrome P450 1B1 is required for 7,12-dimethylbenz(a)-anthracene (DMBA) induced spleen cell immunotoxicity. Toxicol Sci 86, 68-74.

Garcia-Closas, M. Hall, P. Nevanlinna, H. Pooley, K. Morrison, J. Richesson, D. A. Bojesen, S. E. Nordestgaard, B. G. Axelsson, C. K. Arias, J. I. et al. (2008a). Heterogeneity of Breast Cancer Associations with Five Susceptibility Loci by Clinical and Pathological Characteristics. PLoS Genetics 4, e1000054.

Garcia-Closas, M. Hall, P. Nevanlinna, H. Pooley, K. Morrison, J. Richesson, D. A. Bojesen, S. E. Nordestgaard, B. G. Axelsson, C. K. Arias, J. I. et al. (2008b). 
Heterogeneity of breast cancer associations with five susceptibility loci by clinical and pathological characteristics. PLoS Genet 4, el000054.

Geurts, A. M., Cost, G. J., Freyvert, Y., Zeitler, B., Miller, J. C., Choi, V. M., Jenkins, S. S., Wood, A., Cui, X., Meng, X. et al. (2009). Knockout rats via embryo microinjection of zinc-finger nucleases. Science (New York, NY) 325, 433.

Gossen, M. and Bujard, H. (1992). Tight control of gene expression in mammalian cells by tetracycline-responsive promoters. Proceedings of the National Academy of Sciences of the United States of America 89, 5547-51.

Gottardis, M. M., Robinson, S. P., Satyaswaroop, P. G. and Jordan, V. C. (1988). Contrasting actions of tamoxifen on endometrial and breast tumor growth in the athymic mouse. Cancer Res 48, 812-5.

Gould, K. A., Tochacek, M., Schaffer, B. S., Reindl, T. M., Murrin, C. R., Lachel, C. M., VanderWoude, E. A., Pennington, K. L., Flood, L. A., Bynote, K. K. et al. (2004). Genetic determination of susceptibility to estrogen-induced mammary cancer in the ACI rat: mapping of Emcal and Emca2 to chromosomes 5 and 18. Genetics 168, 2113-25.

Gould, M. N. (1986). Inheritance and site of expression of genes controlling susceptibility to mammary cancer in an inbred rat model. Cancer Res 46, 1199-202.

Gould, M. N. (1995). Rodent models for the study of etiology, prevention and treatment of breast cancer. Semin Cancer Biol 6, 147-52.

Gould, M. N. and Clifton, K. H. (1985). Clonogen transplantation assay of mammary and thyroid epithelial cells. In Cell Clones: Manual of Mammalian Techniques, (ed. C. S. Potten and J. H. Hendry), pp. 128-137. Edinburgh, London, Melbourne and New York: Churchill Livingstone.

Grune, T., Brzeski, J., Eberharter, A., Clapier, C. R., Corona, D. F., Becker, P. B. and Muller, C. W. (2003). Crystal structure and functional analysis of a nucleosome recognition module of the remodeling factor ISWI. Mol Cell 12, 449-60.

Guy, C. T., Cardiff, R. D. and Muller, W. J. (1992a). Induction of mammary tumors by expression of polyomavirus middle $T$ oncogene: a transgenic mouse model for metastatic disease. Mol Cell Biol 12, 954-61.

Guy, C. T., Webster, M. A., Schaller, M., Parsons, T. J., Cardiff, R. D. and Muller, W. J. (1992b). Expression of the neu protooncogene in the mammary epithelium of transgenic mice induces metastatic disease. Proceedings of the National Academy of Sciences of the United States of America 89, 10578-82.

Haag, J. D., Hsu, L. C., Newton, M. A. and Gould, M. N. (1996). Allelic imbalance in mammary carcinomas induced by either 7,12-dimethylbenz[a]anthracene or ionizing radiation in rats carrying genes conferring differential susceptibilities to mammary carcinogenesis. Mol Carcinog 17, 134-43. 
Haag, J. D., Newton, M. A. and Gould, M. N. (1992). Mammary carcinoma suppressor and susceptibility genes in the Wistar-Kyoto rat. Carcinogenesis 13, 1933-5.

Haag, J. D., Shepel, L. A., Kolman, B. D., Monson, D. M., Benton, M. E., Watts, K. T., Waller, J. L., Lopez-Guajardo, C. C., Samuelson, D. J. and Gould, M. N. (2003). Congenic rats reveal three independent Copenhagen alleles within the Mcs1 quantitative trait locus that confer resistance to mammary cancer. Cancer Res 63, 5808-12.

Hall, J. M. and McDonnell, D. P. (1999). The estrogen receptor beta-isoform (ERbeta) of the human estrogen receptor modulates ERalpha transcriptional activity and is a key regulator of the cellular response to estrogens and antiestrogens. Endocrinology 140, 5566-78.

Han, W., Woo, J. H., Yu, J. H., Lee, M. J., Moon, H. G., Kang, D. and Noh, D. Y. (2011). Common genetic variants associated with breast cancer in Korean women and differential susceptibility according to intrinsic subtype. Cancer epidemiology, biomarkers \& prevention : a publication of the American Association for Cancer Research, cosponsored by the American Society of Preventive Oncology 20, 793-8.

Hankinson, S. E., Willett, W. C., Manson, J. E., Hunter, D. J., Colditz, G. A., Stampfer, M. J., Longcope, C. and Speizer, F. E. (1995). Alcohol, height, and adiposity in relation to estrogen and prolactin levels in postmenopausal women. $J$ Natl Cancer Inst 87, 1297-302.

Hardell, L. and Sage, C. (2008). Biological effects from electromagnetic field exposure and public exposure standards. Biomed Pharmacother 62, 104-9.

Heery, D. M., Kalkhoven, E., Hoare, S. and Parker, M. G. (1997). A signature motif in transcriptional co-activators mediates binding to nuclear receptors. Nature 387, 733-6.

Hill, S. M., Blask, D. E., Xiang, S., Yuan, L., Mao, L., Dauchy, R. T., Dauchy, E. M., Frasch, T. and Duplesis, T. (2011). Melatonin and associated signaling pathways that control normal breast epithelium and breast cancer. J Mammary Gland Biol Neoplasia 16, 235-45.

Hindorff, L., Institute), J. M. E. B., Wise, A., Junkins, H., Hall, P., Klemm, R. and Manolio, T. (2009). A Catalog of Published Genome-Wide Association Studies, (ed.

Hoover, R., Gray, L. A., Sr., Cole, P. and MacMahon, B. (1976). Menopausal estrogens and breast cancer. The New England journal of medicine 295, 401-5.

Horwitz, K. B., Mockus, M. B. and Lessey, B. A. (1982). Variant T47D human breast cancer cells with high progesterone-receptor levels despite estrogen and antiestrogen resistance. Cell 28, 633-42.

Hsu, L. C., Kennan, W. S., Shepel, L. A., Jacob, H. J., Szpirer, C., Szpirer, J., Lander, E. S. and Gould, M. N. (1994). Genetic identification of Mcs-1, a rat mammary carcinoma suppressor gene. Cancer Res 54, 2765-70. 
Hsueh, A. J., Peck, E. J., Jr. and Clark, J. H. (1976). Control of uterine estrogen receptor levels by progesterone. Endocrinology 98, 438-44.

Hunter, D. J. (2005). Gene-environment interactions in human diseases. Nat Rev Genet 6, 287-98.

Hutchinson, J. N. and Muller, W. J. (2000). Transgenic mouse models of human breast cancer. Oncogene 19, 6130-7.

Isaacs, J. T. (1986). Genetic control of resistance to chemically induced mammary adenocarcinogenesis in the rat. Cancer Res 46, 3958-63.

Isaacs, J. T. (1988). Inheritance of a genetic factor from the Copenhagen rat and the suppression of chemically induced mammary adenocarcinogenesis. Cancer Res 48, 220413.

Jostins, L. and Barrett, J. C. (2011). Genetic risk prediction in complex disease. Hum Mol Genet 20, R182-8.

Justenhoven, C., Hamann, U., Pierl, C. B., Baisch, C., Harth, V., Rabstein, S., Spickenheuer, A., Pesch, B., Bruning, T., Winter, S. et al. (2009). CYP2C19*17 is associated with decreased breast cancer risk. Breast Cancer Res Treat 115, 391-6.

Kelsey, J. L., Gammon, M. D. and John, E. M. (1993). Reproductive factors and breast cancer. Epidemiol Rev 15, 36-47.

Kent, W. J., Sugnet, C. W., Furey, T. S., Roskin, K. M., Pringle, T. H., Zahler, A. M. and Haussler, D. (2002). The human genome browser at UCSC. Genome Res 12, 9961006.

Kester, M. H., Bulduk, S., Tibboel, D., Meinl, W., Glatt, H., Falany, C. N., Coughtrie, M. W., Bergman, A., Safe, S. H., Kuiper, G. G. et al. (2000). Potent inhibition of estrogen sulfotransferase by hydroxylated PCB metabolites: a novel pathway explaining the estrogenic activity of PCBs. Endocrinology 141, 1897-900.

Kleer, C. G., Cao, Q., Varambally, S., Shen, R., Ota, I., Tomlins, S. A., Ghosh, D., Sewalt, R. G., Otte, A. P., Hayes, D. F. et al. (2003). EZH2 is a marker of aggressive breast cancer and promotes neoplastic transformation of breast epithelial cells.

Proceedings of the National Academy of Sciences of the United States of America 100, 11606-11.

Kleinberg, D. L., Ruan, W., Catanese, V., Newman, C. B. and Feldman, M. (1990). Non-lactogenic effects of growth hormone on growth and insulin-like growth factor-I messenger ribonucleic acid of rat mammary gland. Endocrinology 126, 3274-6.

Klinge, C. M. (2000). Estrogen receptor interaction with co-activators and co-repressors. Steroids 65, 227-51.

Kronenberg, H. and Williams, R. H. (2008). Williams textbook of endocrinology. Philadelphia, PA: Saunders/Elsevier. 
Kuiper, G. G., Carlsson, B., Grandien, K., Enmark, E., Haggblad, J., Nilsson, S. and Gustafsson, J. A. (1997). Comparison of the ligand binding specificity and transcript tissue distribution of estrogen receptors alpha and beta. Endocrinology 138, 863-70.

Kurz, S. G., Hansen, K. K., McLaughlin, M. T., Shivaswamy, V., Schaffer, B. S., Gould, K. A., McComb, R. D., Meza, J. L. and Shull, J. D. (2008). Tissue-specific actions of the Ept1, Ept2, Ept6, and Ept9 genetic determinants of responsiveness to estrogens in the female rat. Endocrinology 149, 3850-9.

Lalloo, F. and Evans, D. G. (2012). Familial Breast Cancer. Clin Genet.

Lan, H., Kendziorski, C. M., Haag, J. D., Shepel, L. A., Newton, M. A. and Gould, M. N. (2001). Genetic loci controlling breast cancer susceptibility in the Wistar-Kyoto rat. Genetics 157, 331-9.

Land, C. E., Tokunaga, M., Koyama, K., Soda, M., Preston, D. L., Nishimori, I. and Tokuoka, S. (2003). Incidence of female breast cancer among atomic bomb survivors, Hiroshima and Nagasaki, 1950-1990. Radiat Res 160, 707-17.

Lange, C. A. (2008). Challenges to defining a role for progesterone in breast cancer. Steroids 73, 914-21.

Lanigan, F., O'Connor, D., Martin, F. and Gallagher, W. M. (2007). Molecular links between mammary gland development and breast cancer. Cell Mol Life Sci 64, 3159-84.

Lawther, P. J. and Waller, R. E. (1976). Coal fires, industrial emissions and motor vehicles as sources of environmental carcinogens. IARC Sci Publ, 27-40.

Lee, S. H., Wray, N. R., Goddard, M. E. and Visscher, P. M. (2011). Estimating missing heritability for disease from genome-wide association studies. Am J Hum Genet 88, 294-305.

Lemoine, N. R., Staddon, S., Dickson, C., Barnes, D. M. and Gullick, W. J. (1990). Absence of activating transmembrane mutations in the c-erbB-2 proto-oncogene in human breast cancer. Oncogene 5, 237-9.

Leygue, E., Dotzlaw, H., Watson, P. H. and Murphy, L. C. (1998). Altered estrogen receptor alpha and beta messenger RNA expression during human breast tumorigenesis. Cancer Res 58, 3197-201.

Li, M., Chen, D., Shiloh, A., Luo, J., Nikolaev, A. Y., Qin, J. and Gu, W. (2002). Deubiquitination of p53 by HAUSP is an important pathway for p53 stabilization. Nature 416, 648-53.

Lichtenstein, P., Holm, N. V., Verkasalo, P. K., Iliadou, A., Kaprio, J., Koskenvuo, M., Pukkala, E., Skytthe, A. and Hemminki, K. (2000). Environmental and heritable factors in the causation of cancer--analyses of cohorts of twins from Sweden, Denmark, and Finland. The New England journal of medicine 343, 78-85.

Liehr, J. G. (1990). Genotoxic effects of estrogens. Mutat Res 238, 269-76. 
Liehr, J. G. and Roy, D. (1990). Free radical generation by redox cycling of estrogens. Free Radic Biol Med 8, 415-23.

Lydon, J. P., DeMayo, F. J., Funk, C. R., Mani, S. K., Hughes, A. R., Montgomery, C. A., Jr., Shyamala, G., Conneely, O. M. and O'Malley, B. W. (1995). Mice lacking progesterone receptor exhibit pleiotropic reproductive abnormalities. Genes Dev 9, 226678.

MacMahon, B., Cole, P., Lin, T. M., Lowe, C. R., Mirra, A. P., Ravnihar, B., Salber, E. J., Valaoras, V. G. and Yuasa, S. (1970). Age at first birth and breast cancer risk. Bull World Health Organ 43, 209-21.

Maddison, K. and Clarke, A. R. (2005). New approaches for modelling cancer mechanisms in the mouse. J Pathol 205, 181-93.

Maguire, P., Margolin, S., Skoglund, J., Sun, X. F., Gustafsson, J. A., BorresenDale, A. L. and Lindblom, A. (2005). Estrogen receptor beta (ESR2) polymorphisms in familial and sporadic breast cancer. Breast Cancer Res Treat 94, 145-52.

Marcotte, R. and Muller, W. J. (2008). Signal transduction in transgenic mouse models of human breast cancer--implications for human breast cancer. J Mammary Gland Biol Neoplasia 13, 323-35.

Marian, A. J. (2012). Elements of 'missing heritability'. Curr Opin Cardiol 27, 197-201.

Markel, P., Shu, P., Ebeling, C., Carlson, G. A., Nagle, D. L., Smutko, J. S. and Moore, K. J. (1997). Theoretical and empirical issues for marker-assisted breeding of congenic mouse strains. Nat Genet 17, 280-4.

Martin, P. M., Horwitz, K. B., Ryan, D. S. and McGuire, W. L. (1978). Phytoestrogen interaction with estrogen receptors in human breast cancer cells. Endocrinology 103, 1860-7.

Masson, L. F., Sharp, L., Cotton, S. C. and Little, J. (2005). Cytochrome P-450 1 A1 gene polymorphisms and risk of breast cancer: a HuGE review. Am J Epidemiol 161, 901-15.

Mathers, C., Fat, D. M., Boerma, J. T., World Health Organization. and ebrary Inc. (2008). The global burden of disease 2004 update, (ed. Geneva, Switzerland: World Health Organization.

McCarthy, P. L., Mercer, F. C., Savicky, M. W., Carter, B. A., Paterno, G. D. and Gillespie, L. L. (2008a). Changes in subcellular localisation of MI-ER1 alpha, a novel oestrogen receptor-alpha interacting protein, is associated with breast cancer progression. Br J Cancer 99, 639-46.

McCarthy, P. L., Mercer, F. C., Savicky, M. W. J., Carter, B. A., Paterno, G. D. and Gillespie, L. L. (2008b). Changes in subcellular localisation of MI-ER1 [alpha], a novel oestrogen receptor-[alpha] interacting protein, is associated with breast cancer progression. Br J Cancer 99, 639-646. 
McInerney, E. M., Rose, D. W., Flynn, S. E., Westin, S., Mullen, T. M., Krones, A., Inostroza, J., Torchia, J., Nolte, R. T., Assa-Munt, N. et al. (1998a). Determinants of coactivator LXXLL motif specificity in nuclear receptor transcriptional activation. Genes \& Development 12, 3357-68.

McInerney, E. M., Rose, D. W., Flynn, S. E., Westin, S., Mullen, T. M., Krones, A., Inostroza, J., Torchia, J., Nolte, R. T., Assa-Munt, N. et al. (1998b). Determinants of coactivator LXXLL motif specificity in nuclear receptor transcriptional activation. Genes Dev 12, 3357-68.

Meijers-Heijboer, H., van den Ouweland, A., Klijn, J., Wasielewski, M., de Snoo, A., Oldenburg, R., Hollestelle, A., Houben, M., Crepin, E., van Veghel-Plandsoen, M. et al. (2002). Low-penetrance susceptibility to breast cancer due to CHEK2(*)1100delC in noncarriers of BRCA1 or BRCA2 mutations. Nat Genet 31, 55-9.

Mersch-Sundermann, V., Mochayedi, S., Kevekordes, S., Kern, S. and Wintermann, F. (1993). The genotoxicity of unsubstituted and nitrated polycyclic aromatic hydrocarbons. Anticancer Res 13, 2037-43.

Migliaccio, A., Piccolo, D., Castoria, G., Di Domenico, M., Bilancio, A., Lombardi, M., Gong, W., Beato, M. and Auricchio, F. (1998). Activation of the Src/p21 ras/Erk pathway by progesterone receptor via cross-talk with estrogen receptor. EMBOJ 17, 2008-18.

Milne, R. L., Benitez, J., Nevanlinna, H., Heikkinen, T., Aittomaki, K., Blomqvist, C., Arias, J. I., Zamora, M. P., Burwinkel, B., Bartram, C. R. et al. (2009). Risk of estrogen receptor-positive and -negative breast cancer and single-nucleotide polymorphism 2q35-rs13387042. J Natl Cancer Inst 101, 1012-8.

Miyata, M., Furukawa, M., Takahashi, K., Gonzalez, F. J. and Yamazoe, Y. (2001). Mechanism of 7,12-dimethylbenz[a]anthracene-induced immunotoxicity: role of metabolic activation at the target organ. Jpn J Pharmacol 86, 302-9.

Miyata, M., Kudo, G., Lee, Y. H., Yang, T. J., Gelboin, H. V., Fernandez-Salguero, P., Kimura, S. and Gonzalez, F. J. (1999). Targeted disruption of the microsomal epoxide hydrolase gene. Microsomal epoxide hydrolase is required for the carcinogenic activity of 7,12-dimethylbenz[a] anthracene. J Biol Chem 274, 23963-8.

Modi, B. G., Neustadter, J., Binda, E., Lewis, J., Filler, R. B., Roberts, S. J., Kwong, B. Y., Reddy, S., Overton, J. D., Galan, A. et al. (2012). Langerhans cells facilitate epithelial DNA damage and squamous cell carcinoma. Science (New York, NY) 335, 104-8.

Mokbel, K. (2002). The evolving role of aromatase inhibitors in breast cancer. Int J Clin Oncol 7, 279-83.

Moore, C. J., Bachhuber, A. J. and Gould, M. N. (1983). Relationship of mammary tumor susceptibility, mammary cell-mediated mutagenesis, and metabolism of polycyclic aromatic hydrocarbons in four types of rats. J Natl Cancer Inst 70, 777-84. 
Narod, S. A. and Salmena, L. (2011). BRCA1 and BRCA2 mutations and breast cancer. Discov Med 12, 445-53.

Nathanson, K. L. and Weber, B. L. (2001). "Other" breast cancer susceptibility genes: searching for more holy grail. Hum Mol Genet 10, 715-20.

Neuhausen, S., Gilewski, T., Norton, L., Tran, T., McGuire, P., Swensen, J., Hampel, H., Borgen, P., Brown, K., Skolnick, M. et al. (1996). Recurrent BRCA2 6174delT mutations in Ashkenazi Jewish women affected by breast cancer. Nat Genet 13, 126-8.

Nevanlinna, H. and Bartek, J. (2006). The CHEK2 gene and inherited breast cancer susceptibility. Oncogene 25, 5912-9.

Nijman, I. J., Kuipers, S., Verheul, M., Guryev, V. and Cuppen, E. (2008). A genome-wide SNP panel for mapping and association studies in the rat. BMC Genomics 9, 95 .

O'Malley, B. W. and Kumar, R. (2009). Nuclear receptor coregulators in cancer biology. Cancer Res 69, 8217-22.

Oddoux, C., Struewing, J. P., Clayton, C. M., Neuhausen, S., Brody, L. C., Kaback, M., Haas, B., Norton, L., Borgen, P., Jhanwar, S. et al. (1996). The carrier frequency of the BRCA2 6174delT mutation among Ashkenazi Jewish individuals is approximately 1\%. Nat Genet 14, 188-90.

Ogino, T., Wei, S., Wei, K., Moralejo, D. H., Kose, H., Mizuno, A., Shima, K., Sasaki, Y., Yamada, T. and Matsumoto, K. (2000). Genetic evidence for obesity loci involved in the regulation of body fat distribution in obese type 2 diabetes rat, OLETF. Genomics 70, 19-25.

Onami, S., Ozaki, M., Mortimer, J. E. and Pal, S. K. (2010). Male breast cancer: an update in diagnosis, treatment and molecular profiling. Maturitas 65, 308-14.

Osborne, C. K., Hobbs, K. and Trent, J. M. (1987). Biological differences among MCF-7 human breast cancer cell lines from different laboratories. Breast Cancer Res Treat 9, 111-21.

Osborne, C. K., Shou, J., Massarweh, S. and Schiff, R. (2005). Crosstalk between estrogen receptor and growth factor receptor pathways as a cause for endocrine therapy resistance in breast cancer. Clin Cancer Res 11, 865s-70s.

Paech, K., Webb, P., Kuiper, G. G., Nilsson, S., Gustafsson, J., Kushner, P. J. and Scanlan, T. S. (1997). Differential ligand activation of estrogen receptors ERalpha and ERbeta at AP1 sites. Science (New York, NY) 277, 1508-10.

Pan, C., Kumar, C., Bohl, S., Klingmueller, U. and Mann, M. (2009). Comparative proteomic phenotyping of cell lines and primary cells to assess preservation of cell typespecific functions. Mol Cell Proteomics 8, 443-50. 
Paterno, G. D., Li, Y., Luchman, H. A., Ryan, P. J. and Gillespie, L. L. (1997). cDNA cloning of a novel, developmentally regulated immediate early gene activated by fibroblast growth factor and encoding a nuclear protein. $J$ Biol Chem 272, 25591-5.

Paterno, G. D., Mercer, F. C., Chayter, J. J., Yang, X., Robb, J. D. and Gillespie, L. L. (1998). Molecular cloning of human er1 cDNA and its differential expression in breast tumours and tumour-derived cell lines. Gene 222, 77-82.

Pelucchi, C., Tramacere, I., Boffetta, P., Negri, E. and La Vecchia, C. (2011). Alcohol consumption and cancer risk. Nutr Cancer 63, 983-90.

Peto, R. (2000). Cancer, genes, and the environment. The New England journal of medicine 343, 1495; discussion 1495-6.

Pettersson, K., Delaunay, F. and Gustafsson, J. A. (2000). Estrogen receptor beta acts as a dominant regulator of estrogen signaling. Oncogene 19, 4970-8.

Pettersson, K., Grandien, K., Kuiper, G. G. and Gustafsson, J. A. (1997). Mouse estrogen receptor beta forms estrogen response element-binding heterodimers with estrogen receptor alpha. Mol Endocrinol 11, 1486-96.

Pharoah, P. D., Antoniou, A., Bobrow, M., Zimmern, R. L., Easton, D. F. and Ponder, B. A. (2002). Polygenic susceptibility to breast cancer and implications for prevention. Nat Genet 31, 33-6.

Pharoah, P. D., Antoniou, A. C., Easton, D. F. and Ponder, B. A. (2008). Polygenes, risk prediction, and targeted prevention of breast cancer. The New England journal of medicine 358, 2796-803.

Phillips, D. H. (1999). Polycyclic aromatic hydrocarbons in the diet. Mutat Res 443, 13947.

Pijpe, A., Andrieu, N., Easton, D. F., Kesminiene, A., Cardis, E., Nogues, C., Gauthier-Villars, M., Lasset, C., Fricker, J. P., Peock, S. et al. (2012). Exposure to diagnostic radiation and risk of breast cancer among carriers of BRCA1/2 mutations: retrospective cohort study (GENE-RAD-RISK). BMJ (Clinical research ed) 345, e5660.

Pike, M. C., Krailo, M. D., Henderson, B. E., Casagrande, J. T. and Hoel, D. G. (1983). 'Hormonal' risk factors, 'breast tissue age' and the age-incidence of breast cancer. Nature 303, 767-70.

Pike, M. C., Spicer, D. V., Dahmoush, L. and Press, M. F. (1993). Estrogens, progestogens, normal breast cell proliferation, and breast cancer risk. Epidemiol Rev 15, 17-35.

Pollard, J. W. (2004). Tumour-educated macrophages promote tumour progression and metastasis. Nat Rev Cancer 4, 71-8.

Prentice, R. L., Huang, Y., Hinds, D. A., Peters, U., Pettinger, M., Cox, D. R., Beilharz, E., Chlebowski, R. T., Rossouw, J. E., Caan, B. et al. (2009). Variation in 
the FGFR2 gene and the effects of postmenopausal hormone therapy on invasive breast cancer. Cancer Epidemiol Biomarkers Prev 18, 3079-85.

Preston-Martin, S., Pike, M. C., Ross, R. K. and Henderson, B. E. (1993).

Epidemiologic evidence for the increased cell proliferation model of carcinogenesis.

Environ Health Perspect 101 Suppl 5, 137-8.

Pylkas, K., Vuorela, M., Otsukka, M., Kallioniemi, A., Jukkola-Vuorinen, A. and Winqvist, R. (2012). Rare copy number variants observed in hereditary breast cancer cases disrupt genes in estrogen signaling and TP53 tumor suppression network. PLoS Genet 8, e1002734.

Qiu, C., Yu, M., Shan, L. and Snyderwine, E. G. (2003a). Allelic imbalance and altered expression of genes in chromosome $2 q 11-2 q 16$ from rat mammary gland carcinomas induced by 2-amino-1-methyl-6-phenylimidazo[4,5-b]pyridine. Oncogene 22, 1253-60.

Qiu, C., Yu, M., Shan, L. and Snyderwine, E. G. (2003b). Allelic imbalance and altered expression of genes in chromosome $2 q 11-2 q 16$ from rat mammary gland carcinomas induced by 2-amino-1-methyl-6-phenylimidazo[1sqb]4,5-b[rsqb]pyridine. Oncogene 22, 1253-1260.

Rahman, N., Seal, S., Thompson, D., Kelly, P., Renwick, A., Elliott, A., Reid, S., Spanova, K., Barfoot, R., Chagtai, T. et al. (2007). PALB2, which encodes a BRCA2interacting protein, is a breast cancer susceptibility gene. Nat Genet 39, 165-7.

Rhodes, D. R., Yu, J., Shanker, K., Deshpande, N., Varambally, R., Ghosh, D., Barrette, T., Pandey, A. and Chinnaiyan, A. M. (2004). ONCOMINE: a cancer microarray database and integrated data-mining platform. Neoplasia 6, 1-6.

Ring, A. and Dowsett, M. (2004). Mechanisms of tamoxifen resistance. Endocr Relat Cancer 11, 643-58.

Roa, B. B., Boyd, A. A., Volcik, K. and Richards, C. S. (1996). Ashkenazi Jewish population frequencies for common mutations in BRCA1 and BRCA2. Nat Genet 14, 185-7.

Rogan, E. G., Badawi, A. F., Devanesan, P. D., Meza, J. L., Edney, J. A., West, W. W., Higginbotham, S. M. and Cavalieri, E. L. (2003). Relative imbalances in estrogen metabolism and conjugation in breast tissue of women with carcinoma: potential biomarkers of susceptibility to cancer. Carcinogenesis 24, 697-702.

Ronckers, C. M., Erdmann, C. A. and Land, C. E. (2005). Radiation and breast cancer: a review of current evidence. Breast Cancer Res 7, 21-32.

Rossouw, J. E., Anderson, G. L., Prentice, R. L., LaCroix, A. Z., Kooperberg, C., Stefanick, M. L., Jackson, R. D., Beresford, S. A., Howard, B. V., Johnson, K. C. et al. (2002). Risks and benefits of estrogen plus progestin in healthy postmenopausal 
women: principal results From the Women's Health Initiative randomized controlled trial. JAMA : the journal of the American Medical Association 288, 321-33.

Rozen, S. and Skaletsky, H. (2000). Primer3 on the WWW for general users and for biologist programmers. Methods Mol Biol 132, 365-86.

Ruan, W., Monaco, M. E. and Kleinberg, D. L. (2005). Progesterone stimulates mammary gland ductal morphogenesis by synergizing with and enhancing insulin-like growth factor-I action. Endocrinology 146, 1170-8.

Russo, J. and Russo, I. H. (1994). Toward a physiological approach to breast cancer prevention. Cancer Epidemiol Biomarkers Prev 3, 353-64.

Saar, K., Beck, A., Bihoreau, M. T., Birney, E., Brocklebank, D., Chen, Y., Cuppen, E., Demonchy, S., Dopazo, J., Flicek, P. et al. (2008). SNP and haplotype mapping for genetic analysis in the rat. Nat Genet 40, 560-6.

Safe, S. H. (1997). Is there an association between exposure to environmental estrogens and breast cancer? Environ Health Perspect 105 Suppl 3, 675-8.

Saitoh, M., Ohmichi, M., Takahashi, K., Kawagoe, J., Ohta, T., Doshida, M., Takahashi, T., Igarashi, H., Mori-Abe, A., Du, B. et al. (2005). Medroxyprogesterone acetate induces cell proliferation through up-regulation of cyclin D1 expression via phosphatidylinositol 3-kinase/Akt/nuclear factor-kappaB cascade in human breast cancer cells. Endocrinology 146, 4917-25.

Samuelson, D. J., Haag, J. D., Lan, H., Monson, D. M., Shultz, M. A., Kolman, B. D. and Gould, M. N. (2003). Physical evidence of Mes5, a QTL controlling mammary carcinoma susceptibility, in congenic rats. Carcinogenesis 24, 1455-60.

Samuelson, D. J., Hesselson, S. E., Aperavich, B. A., Zan, Y., Haag, J. D., TrenthamDietz, A., Hampton, J. M., Mau, B., Chen, K. S., Baynes, C. et al. (2007). Rat Mcs5a is a compound quantitative trait locus with orthologous human loci that associate with breast cancer risk. Proceedings of the National Academy of Sciences of the United States of America 104, 6299-304.

Santen, R. J., Brodie, H., Simpson, E. R., Siiteri, P. K. and Brodie, A. (2009). History of aromatase: saga of an important biological mediator and therapeutic target. Endocr Rev 30, 343-75.

Schechter, A. L., Hung, M. C., Vaidyanathan, L., Weinberg, R. A., Yang-Feng, T. L., Francke, U., Ullrich, A. and Coussens, L. (1985). The neu gene: an erbBhomologous gene distinct from and unlinked to the gene encoding the EGF receptor. Science (New York, $N Y$ ) 229, 976-8.

Seal, S., Thompson, D., Renwick, A., Elliott, A., Kelly, P., Barfoot, R., Chagtai, T., Jayatilake, H., Ahmed, M., Spanova, K. et al. (2006). Truncating mutations in the Fanconi anemia J gene BRIP1 are low-penetrance breast cancer susceptibility alleles. Nat Genet 38, 1239-41. 
Shepel, L. A., Lan, H., Haag, J. D., Brasic, G. M., Gheen, M. E., Simon, J. S., Hoff, P., Newton, M. A. and Gould, M. N. (1998). Genetic identification of multiple loci that control breast cancer susceptibility in the rat. Genetics 149, 289-99.

Shull, J. D., Pennington, K. L., Reindl, T. M., Snyder, M. C., Strecker, T. E., Spady, T. J., Tochacek, M. and McComb, R. D. (2001). Susceptibility to estrogen-induced mammary cancer segregates as an incompletely dominant phenotype in reciprocal crosses between the ACI and Copenhagen rat strains. Endocrinology 142, 5124-30.

Shull, J. D., Spady, T. J., Snyder, M. C., Johansson, S. L. and Pennington, K. L. (1997). Ovary-intact, but not ovariectomized female ACI rats treated with 17betaestradiol rapidly develop mammary carcinoma. Carcinogenesis 18, 1595-601.

Sica, A., Schioppa, T., Mantovani, A. and Allavena, P. (2006). Tumour-associated macrophages are a distinct $\mathrm{M} 2$ polarised population promoting tumour progression: potential targets of anti-cancer therapy. Eur J Cancer 42, 717-27.

Singletary, K. W. and McNary, M. Q. (1992). Effect of moderate ethanol consumption on mammary gland structural development and DNA synthesis in the female rat. Alcohol 9, 95-101.

Sinha, R., Gustafson, D. R., Kulldorff, M., Wen, W. Q., Cerhan, J. R. and Zheng, W. (2000). 2-amino-1-methyl-6-phenylimidazo[4,5-b]pyridine, a carcinogen in hightemperature-cooked meat, and breast cancer risk. J Natl Cancer Inst 92, 1352-4.

Smith, P., McGuffog, L., Easton, D. F., Mann, G. J., Pupo, G. M., Newman, B., Chenevix-Trench, G., Szabo, C., Southey, M., Renard, H. et al. (2006). A genome wide linkage search for breast cancer susceptibility genes. Genes Chromosomes Cancer 45, 646-55.

Smits, B., Sharma, D., Samuelson, D., Woditschka, S., Mau, B., Haag, J. and Gould, · M. (2011a). The non-protein coding breast cancer susceptibility locus Mcs5a acts in a non-mammary cell-autonomous fashion through the immune system and modulates $\mathrm{T}$ cell homeostasis and functions. Breast Canicer Research 13, R81.

Smits, B. M., Sharma, D., Samuelson, D. J., Woditschka, S., Mau, B., Haag, J. D. and Gould, M. N. (2011b). The non-protein coding breast cancer susceptibility locus Mcs5a acts in a non-mammary cell-autonomous fashion through the immune system and modulates T-cell homeostasis and functions. Breast Cancer Res 13, R81.

Solari, F., Bateman, A. and Ahringer, J. (1999). The Caenorhabditis elegans genes egl27 and egr-1 are similar to MTA1, a member of a chromatin regulatory complex, and are redundantly required for embryonic patterning. Development 126, 2483-94.

Stacey, S. N., Manolescu, A., Sulem, P., Thorlacius, S., Gudjonsson, S. A., Jonsson, G. F., Jakobsdottir, M., Bergthorsson, J. T., Gudmundsson, J., Aben, K. K. et al. (2008). Common variants on chromosome $5 \mathrm{p} 12$ confer susceptibility to estrogen receptor-positive breast cancer. Nat Genet 40, 703-6. 
Stewart, T. A., Pattengale, P. K. and Leder, P. (1984). Spontaneous mammary adenocarcinomas in transgenic mice that carry and express $\mathrm{MTV} / \mathrm{myc}$ fusion genes. Cell 38, 627-37.

Struewing, J. P., Abeliovich, D., Peretz, T., Avishai, N., Kaback, M. M., Collins, F. S. and Brody, L. C. (1995). The carrier frequency of the BRCA1 185delAG mutation is approximately 1 percent in Ashkenazi Jewish individuals. Nat Genet 11, 198-200.

Thomas, D. (2010). Methods for investigating gene-environment interactions in candidate pathway and genome-wide association studies. Annu Rev Public Health 31, 21 36.

Thomas, G., Jacobs, K. B., Kraft, P., Yeager, M., Wacholder, S., Cox, D. G., Hankinson, S. E., Hutchinson, A., Wang, Z., Yu, K. et al. (2009). A multistage genome-wide association study in breast cancer identifies two new risk alleles at $1 \mathrm{p} 11.2$ and 14q24.1 (RAD51L1). Nat Genet 41, 579-84.

Thorne, L. B., Grant, A. L., Paterno, G. D. and Gillespie, L. L. (2005). Cloning and characterization of the mouse ortholog of mi-erl. DNA Seq 16, 237-40.

Todorovic, R., Ariese, F., Devanesan, P., Jankowiak, R., Small, G. J., Rogan, E. and Cavalieri, E. (1997). Determination of benzo[a]pyrene- and 7,12-

dimethylbenz[a]anthracene-DNA adducts formed in rat mammary glands. Chem Res Toxicol 10, 941-7.

Travis, R. C., Reeves, G. K., Green, J., Bull, D., Tipper, S. J., Baker, K., Beral, V., Peto, R., Bell, J., Zelenika, D. et al. (2010). Gene-environment interactions in 7610 women with breast cancer: prospective evidence from the Million Women Study. Lancet 375, 2143-51.

Trimboli, A. J., Cantemir-Stone, C. Z., Li, F., Wallace, J. A., Merchant, A., Creasap, N., Thompson, J. C., Caserta, E., Wang, H., Chong, J. L. et al. (2009). Pten in stromal fibroblasts suppresses mammary epithelial tumours. Nature 461, 1084-91.

Tseng, L. and Gurpide, E. (1975). Effects of progestins on estradiol receptor levels in human endometrium. J Clin Endocrinol Metab 41, 402-4.

Tsutsui, T., Maizumi, H., McLachlan, J. A. and Barrett, J. C. (1983). Aneuploidy induction and cell transformation by diethylstilbestrol: a possible chromosomal mechanism in carcinogenesis. Cancer Res 43, 3814-21.

Tsutsui, T., Tamura, Y., Suzuki, A., Hirose, Y., Kobayashi, M., Nishimura, H., Metzler, M. and Barrett, J. C. (2000). Mammalian cell transformation and aneuploidy induced by five bisphenols. Int J Cancer 86, 151-4.

Turnbull, C., Ahmed, S., Morrison, J., Pernet, D., Renwick, A., Maranian, M., Seal, S., Ghoussaini, M., Hines, S., Healey, C. S. et al. (2010). Genome-wide association study identifies five new breast cancer susceptibility loci. Nat Genet 42, 504-7. 
Vahteristo, P., Bartkova, J., Eerola, H., Syrjakoski, K., Ojala, S., Kilpivaara, O., Tamminen, A., Kononen, J., Aittomaki, K., Heikkila, P. et al. (2002). A CHEK2 genetic variant contributing to a substantial fraction of familial breast cancer. Am J Hum Genet 71, 432-8.

van Boxtel, R., Kuiper, R. V., Toonen, P. W., van Heesch, S., Hermsen, R., de Bruin, A. and Cuppen, E. (2011). Homozygous and Heterozygous p53 Knockout Rats Develop Metastasizing Sarcomas with High Frequency. The American Journal of Pathology 179, $1616-1622$.

Vargova, K., Curik, N., Burda, P., Basova, P., Kulvait, V., Pospisil, V., Savvulidi, F., Kokavec, J., Necas, E., Berkova, A. et al. (2011). MYB transcriptionally regulates the miR-155 host gene in chronic lymphocytic leukemia. Blood 117, 3816-25.

Wang, B., Kennan, W. S., Yasukawa-Barnes, J., Lindstrom, M. J. and Gould, M. N. (1991). Overcoming the activity of mammary carcinoma suppressor gene in Copenhagen rats by v-H-ras oncogene transfer into mammary epithelial cells in situ. Cancer Res 51, 5298-303.

Wang, S., Saboorian, M. H., Frenkel, E., Hynan, L., Gokaslan, S. T. and Ashfaq, R. (2000). Laboratory assessment of the status of Her-2/neu protein and oncogene in breast cancer specimens: comparison of immunohistochemistry assay with fluorescence in situ hybridisation assays. $J$ Clin Pathol 53, 374-81.

Wang, T. C., Cardiff, R. D., Zukerberg, L., Lees, E., Arnold, A. and Schmidt, E. V. (1994). Mammary hyperplasia and carcinoma in MMTV-cyclin D1 transgenic mice.

Nature 369, 669-71.

Williams, J. M. and Daniel, C. W. (1983). Mammary ductal elongation: differentiation of myoepithelium and basal lamina during branching morphogenesis. Dev Biol 97, 274$90^{\circ}$

Wooster, R. and Weber, B. L. (2003). Breast and ovarian cancer. The New England journal of medicine 348, 2339-47.

Yu, J., Rhodes, D. R., Tomlins, S. A., Cao, X., Chen, G., Mehra, R., Wang, X., Ghosh, D., Shah, R. B., Varambally, S. et al. (2007). A polycomb repression signature in metastatic prostate cancer predicts cancer outcome. Cancer Res 67, 10657-63.

Zhang, R., Haag, J. D. and Gould, M. N. (1990). Site of expression and biological function of the rat mammary carcinoma suppressor gene. Carcinogenesis 11, 1765-70.

Zhang, Y. and Chen, H. (2011). Genistein, an epigenome modifier during cancer prevention. Epigenetics 6, 888-91.

Zheng, W., Deitz, A. C., Campbell, D. R., Wen, W. Q., Cerhan, J. R., Sellers, T. A., Folsom, A. R. and Hein, D. W. (1999). N-acetyltransferase 1 genetic polymorphism, cigarette smoking, well-done meat intake, and breast cancer risk. Cancer Epidemiol Biomarkers Prev 8, 233-9. 
Zheng, W., Gustafson, D. R., Sinha, R., Cerhan, J. R., Moore, D., Hong, C. P., Anderson, K. E., Kushi, L. H., Sellers, T. A. and Folsom, A. R. (1998). Well-done meat intake and the risk of breast cancer. $J$ Natl Cancer Inst 90, 1724-9.

Zheng, W. and Lee, S. A. (2009). Well-done meat intake, heterocyclic amine exposure, and cancer risk. Nutr Cancer 61, 437-46.

Zheng, W., Wen, W., Gao, Y. T., Shyr, Y., Zheng, Y., Long, J., Li, G., Li, C., Gu, K., Cai, Q. et al. (2010). Genetic and clinical predictors for breast cancer risk assessment and stratification among Chinese women. Journal of the National Cancer Institute 102, 972-81.

Zheng, W., Wen, W. Q., Gustafson, D. R., Gross, M., Cerhan, J. R. and Folsom, A. R. (2002). GSTM1 and GSTT1 polymorphisms and postmenopausal breast cancer risk. Breast Cancer Res Treat 74, 9-16. 
APPENDIX I 


\section{Introduction}

During the course of my dissertation work some supporting experiments were not completed or were omitted into dissertation chapters as to not distract from the focus of the project. Despite this, some of the experiments and resulting data not included in the main body of this dissertation contributed to the conclusions of the work presented here and therefore are shown in the appendix. Below, these data will be explained in the context of the previous work described.

\section{Endogenous Control for Taqman Gene Expression Studies}

\section{Introduction}

Early attempts to measure gene expression of rat $M c s l b$ transcripts were performed using Gapdh as an endogenous control. However, the variability in Gapdh expression was high between samples and this was believed to be due to poor RNA quality. RNA quality was within acceptable limits $(\sim 1.8)$ for absorbance ratios at $260 \mathrm{~nm}$ and $280 \mathrm{~nm}$ when analyzed on a Nanodrop 2000 (Thermo Fisher Scientific); regardless, attempts were made to improve RNA isolation times and conditions but resulted in no change in the high variability in Gapdh expression between samples. I further analyzed RNA quality using RNA Pico Chips and an Agilent Bioanalyzer (Agilent, Santa Clara, CA). Based on RNA Integrity Numbers (RIN) determined by Bioanalyzer data, RNA was determined to not be compromised and that variability was due to variations in Gapdh expression itself and was not a good endogenous control candidate. Thus, I sought to identify a better endogenous control by measuring a panel of endogenous control gene candidates in WF and resistant WF.COP mammary glands. 
Materials and Methods

\section{$R N A$ clean-up using sodium acetate}

As stated above, Gapdh expression was highly variable between samples in initial gene expression assays. I initially believed this was due to degraded or contaminated RNA. To improve RNA quality I made some changes to the RNA isolation protocol. First, fewer samples were isolated in individual sessions to reduce the amount of time the RNA isolates were on ice. Total RNA was extracted with TRI-Reagent (Molecular Research Center) followed by standard chloroform/ethanol precipitation. To reduce possible solvent and DNA contamination RNA samples were further processed by a $1 / 10$ $\mathrm{v} / \mathrm{v} 3 \mathrm{M}$ sodium acetate and $2.5 \mathrm{x}$ v/v $100 \%$ ethanol wash on ice for 10 minutes followed by $80 \%$ ethanol wash followed by Turbo DNase (Life Technologies) treatment. Total RNA quantity and quality were measured with a Nanodrop 1000 (Fisher Scientific) and a Bioanalyzer with RNA 6000 NanoChips (Agilent).

\section{Endogenous Control Array}

To determine a good endogenous control candidate for the $M c s l b$ gene expression studies, I used an endogenous control array micro-fluidic card (Life Technologies). The card contains 16 different endogenous control probe/primer sets (Table S1) preloaded into the card. cDNA was prepared from four mammary carcinoma resistant WF.COP and four susceptible WF mammary gland samples and normalized to $2 \mu \mathrm{g}$. They were loaded in triplicate into the endogenous control array card according to manufacturer specifications, centrifuged and run on an ABI Prism 7900 HT using standard conditions. 


\section{Statistical Analysis}

Endogenous control data were analyzed using SDS software (Life Technologies) and StatView (SAS Institute). Geometric means were calculated for each sample and Mann-Whitney tests were used to analyze expression between genotypes.

\section{$\underline{\text { Results }}$}

$3 M$ sodium acetate results in higher quality $R N A$

Total RNA had higher RIN and 28S and $18 \mathrm{~S}$ bands were stronger when analyzed with the Bioanalyzer. However, differences in Gapdh expression between WF and resistant mammary gland samples did not change following reducing time on ice or by sodium acetate cleanup.

Rplp2 is a good endogenous control gene for gene expression assays in rat mammary gland samples

- Expression levels were highly variable between the genes in the panel (Figure $\mathrm{S} 1$ ). Ct values for most of the $M c s l b$ target transcripts were in a range of $\sim 20-25$ cycles (data not shown). Therefore, genes with mean $\mathrm{Ct}$ values outside the range of $20-25$ cycles were omitted from further analysis. Candidate control genes were further analyzed for variance between replicates and between samples. Samples with SD higher than $0.5 \mathrm{Ct}$ were excluded.

Seven genes in the panel fell inside the allowable $\mathrm{Ct}$ range: $A c t b l 2, A r b p, B 2 m$, Ppia, Ppib, Rplp2, and Ubc (Table S2). Of these, only Rplp2 and Ubc had SDs below 0.5 cycles for both genotype groups. Ubc was differentially expressed between WF and 


\section{Table S1:}

Endogenous control genes contained on ABI Endogenous Control Array MicroFluidic Card

\begin{tabular}{lcc}
\hline Name (Alternate Name) & Gene Symbol & ABI product reference number \\
\hline 18S & $18 \mathrm{~S}$ & Hs 99999901 \\
Beta-actin & Actb & Rn00667869 \\
Attachment region binding protein & Arbp & Rn00821065 \\
Beta-2 microglobulin & B2m & Rn00560865 \\
Glyceraldehyde-3-phosphate dehydrogenase & Gapdh & Rn99999916 \\
Beta-glucuronidase & Gusb & Rn00566655 \\
Hydroxymethylbilane synthase & Hmbs & Rn00565886 \\
Hypoxanthine phosphoribosyltransferase 1 & Hprt & Rn01527840 \\
Phosphoglycerate kinase 1 & Pgk1 & Rn00821429 \\
Peptidylprolyl isomerase A (Cyclophilin A) & Ppia & Rn00690933 \\
Peptidylprolyl isomerase B (Cyclophilin B) & Ppib & Rn00574762 \\
Ribosomal protein, large, P2 & Rplp2 & Rn014799271 \\
TATA box binding protein & Tbp & Rn01455648 \\
Transferrin receptor (p90, CD71) & Tfrc & Rn01474695 \\
Ubiquitin C & Ubc & Rn017898121 \\
Tyrosine 3-monooxygenase/tryptophan 5-monooxygenase activation protein. zeta polypeptide & Ywhaz & Rn00755072 \\
\hline
\end{tabular}




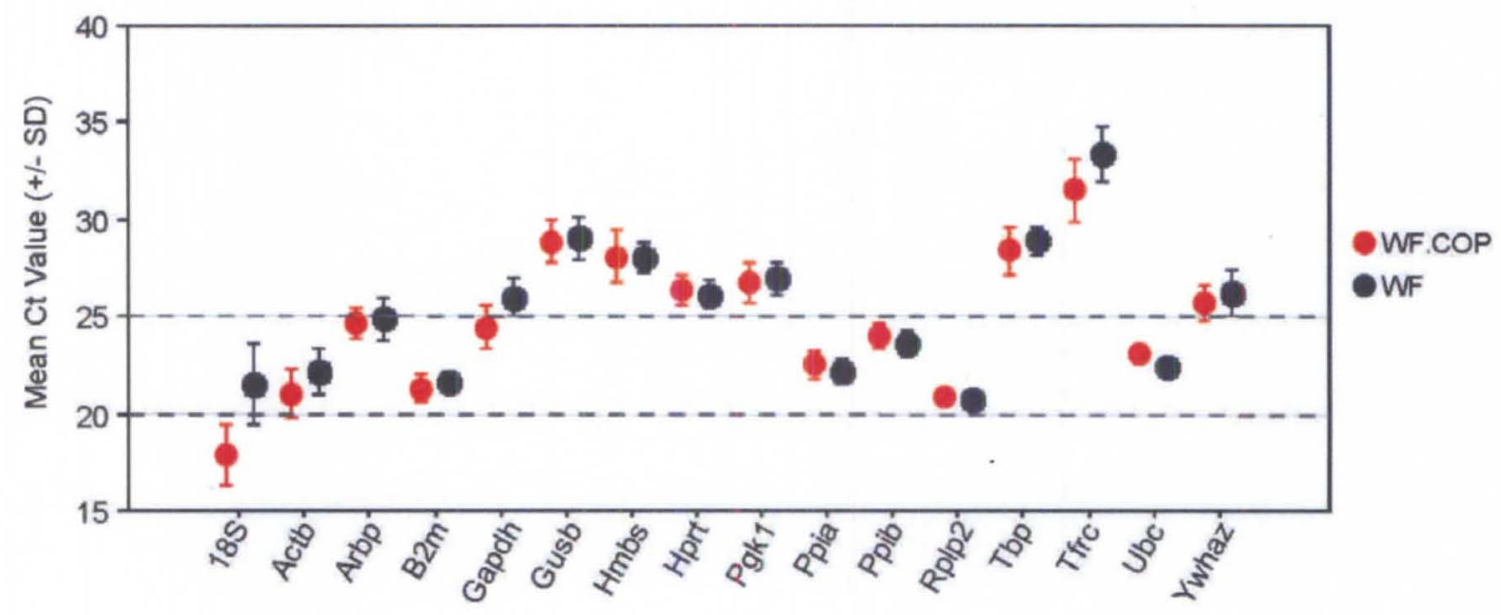

Figure S1: Mean Ct Values for Genes tested in ABI Endogenous Control Array. The geometric mean of the $\mathrm{Ct}$ value for each genotype is plotted. Error bars represent SD. The dashed line delineates the $\mathrm{Ct}$ value range desired for an optimal endogenous control. 
Table S2:

Results of ABI Endogenous Control Array Micro-Fluidic Assay

\begin{tabular}{cccc}
\hline & \multicolumn{2}{c}{ Mean Ct value \pm S.D. } & \\
\cline { 2 - 3 } Gene Symbol & WF & WF.COP & P value $^{\mathbf{a}}$ \\
\hline 18S & $21.5 \pm 2.3$ & $17.9 \pm 1.8$ & 0.0433 \\
Actb & $22.2 \pm 1.3$ & $21.0 \pm 1.3$ & 0.2482 \\
Arbp & $24.8 \pm 1.2$ & $24.6 \pm 0.8$ & 0.3865 \\
B2m & $21.6 \pm 0.6$ & $21.3 \pm 0.8$ & 0.7728 \\
Gapdh & $26.0 \pm 1.1$ & $24.4 \pm 1.2$ & 0.1489 \\
Gusb & $29.0 \pm 1.2$ & $28.8 \pm 1.2$ & $>.9999$ \\
Hmbs & $28.0 \pm 0.9$ & $28.1 \pm 1.5$ & 0.7728 \\
Hprt & $26.1 \pm 0.7$ & $26.4 \pm 0.9$ & 0.7728 \\
Pgk1 & $26.9 \pm 1.0$ & $26.7 \pm 1.1$ & 0.7728 \\
Ppia & $22.2 \pm 0.7$ & $22.5 \pm 0.8$ & 0.7728 \\
Ppib & $23.6 \pm 0.7$ & $24.0 \pm 0.7$ & 0.3865 \\
Rplp2 & $20.7 \pm 0.5$ & $20.8 \pm 0.4$ & 0.7728 \\
Tbp & $28.9 \pm 0.7$ & $28.4 \pm 1.3$ & $>.9999$ \\
Tfrc & $33.3 \pm 1.6$ & $31.5 \pm 1.8$ & 0.1489 \\
Ubc & $22.4 \pm 0.5$ & $23.0 \pm 0.2$ & 0.0433 \\
Ywhaz & $26.2 \pm 1.3$ & $25.7 \pm 1.0$ & 0.7728 \\
\hline
\end{tabular}

${ }^{a}$ Non-parametric Mann-Whitney test 
resistant WF.COP samples $(\mathrm{P}=0.0433)$; however, Rplp2 was not different between genotypes $(\mathrm{P}=0.7728)$. Therefore, Rplp2 was used in all subsequent gene expression experiments. 
APPENDIX II 


\section{LIST OF ABBREVIATIONS AND SYMBOLS}

\begin{tabular}{|c|c|}
\hline $17 \beta$-HSD & $17 \beta$-hydroxysteroid dehydrogenase \\
\hline $18 S$ & $18 \mathrm{~S}$ \\
\hline $\mathrm{ACF}$ & Adherent cell fraction \\
\hline $\mathrm{ACI}$ & $\mathrm{ACl}$ rat strain \\
\hline$A c t b$ & Beta-actin \\
\hline $\mathrm{AI}$ & Aromatase inhibitor \\
\hline Ankrd55 & Ankyrin repeat domain 55 \\
\hline$A r b p$ & Attachment region binding protein \\
\hline $\mathrm{B}[\mathrm{a}] \mathrm{P}$ & Benzo(a)pyrene \\
\hline$B 2 m$ & Beta-2 microglobulin \\
\hline $\mathrm{BAC}$ & Bacterial artificial chromosome \\
\hline $\mathrm{BC}$ & Backcross generation \\
\hline $\mathrm{BN}$ & Brown Norway rat strain \\
\hline bp & Base pairs \\
\hline BPA & Bisphenol A \\
\hline C5orf35 & $\begin{array}{l}\text { Chromosome } 5 \text { open reading frame } 35 \text { (SET domain- } \\
\text { containing protein 9) }\end{array}$ \\
\hline cDNA & Complementary DNA \\
\hline gDNA & Genomic DNA \\
\hline$C h r$ & Chromosome \\
\hline $\mathrm{CI}$ & Confidence Interval \\
\hline
\end{tabular}




\begin{tabular}{|c|c|}
\hline CK1 & Casein kinase 1 \\
\hline $\mathrm{cM}$ & CentiMorgan \\
\hline $\mathrm{CNV}$ & Copy number variant \\
\hline $\mathrm{COP} / \mathrm{Hsd}$ & Copenhagen (Harlan Sprague Dawley) rat strain \\
\hline CYP & Cytochrome P450 \\
\hline DCIS & Ductal carcinoma in situ \\
\hline$D d x 4$ & DEAD (Asp-Glu-Ala-Asp) box polypeptide 4 \\
\hline DES & Diethylstilbestrol \\
\hline DMBA & 7,12 Dimethylbenz(a)anthracene \\
\hline DMEM & Dulbecco's Modified Eagle Medium \\
\hline DNase & Deoxyribonuclease I \\
\hline dATP & Deoxyadenosine triphosphate \\
\hline dCTP & Deoxycytidine triphosphate \\
\hline dGTP & Deoxyguanosine triphosphate \\
\hline dTTP & Deoxythymidine triphosphate \\
\hline dNTP & Deoxynucelotide triphosphate \\
\hline E1 & Estrone \\
\hline E2 & Estradiol \\
\hline E3 & Estriol \\
\hline ECM & Extracellular matrix \\
\hline EDTA & Ethylenediaminetetraacetic acid \\
\hline ELM2 & Egl-27 and MTA1 homology 2 domain \\
\hline
\end{tabular}




\begin{tabular}{|c|c|}
\hline ERE & Estrogen Response Element \\
\hline $\mathrm{ER} \alpha$ & Estrogen receptor alpha \\
\hline $\mathrm{ER} \beta$ & Estrogen receptor beta \\
\hline Gapdh & Glyceraldehyde-3-phosphate dehydrogenase \\
\hline Gpbpl & GC-rich promoter binding protein 1 \\
\hline Gusb & Beta-glucuronidase \\
\hline GWAS & Genome-wide association study \\
\hline $\mathrm{HCA}$ & Heterocyclic amine \\
\hline HDAC & Histone deacetylase \\
\hline$H m b s$ & Hydroxymethylbilane synthase \\
\hline Hprt & Hypoxanthine phosphoribosyltransferase 1 \\
\hline HR & Hazard Ratio \\
\hline HRT & Hormone replacement therapy \\
\hline IACUC & Institutional Animal Care and Use Committee \\
\hline IDBC & Invasive ductal breast carcinoma \\
\hline Il $31 r a$ & Interleukin 31 receptor $\mathrm{A}$ \\
\hline Il6st & Interleukin 6 signal transducer \\
\hline ILBC & Invasive lobular breast carcinoma \\
\hline indel & Nucleotide Insertion/Deletion variant \\
\hline LD & Linkage disequilibrium \\
\hline LTR & Long terminal repeat \\
\hline LxxLL & Leu-Xaa-Xaa-Leu-Leu domain \\
\hline
\end{tabular}




\begin{tabular}{|c|c|}
\hline MAF & Minor allele frequency \\
\hline Map $3 k 1$ & Mitogen-activated protein kinase kinase kinase 1 \\
\hline Mcs & Mammary carcinoma susceptibility locus \\
\hline MEC & Mammary epithelial cell \\
\hline $\mathrm{MHC}$ & Major histocompatibility complex \\
\hline Mier3 & Mesoderm induction early response 1 , family member 3 \\
\hline miRNA & MicroRNA \\
\hline MMTV & Murine mammary tumor virus \\
\hline MTA1 & Metastatic tumor antigen 1 \\
\hline MWS & Million Women Study \\
\hline $\mathrm{NCI}$ & National Cancer Institute \\
\hline nitro-PAH & Nitrated polycyclic aromatic hydrocarbon metabolite \\
\hline NMU & $N$-Nitroso- $N$-methylurea \\
\hline NSABP & National Surgical Adjuvant Breast and Bowel Project \\
\hline NuRD & Nucleosome remodeling and histone deacetylase complex \\
\hline OR & Odds ratio \\
\hline ORF & Open reading frame \\
\hline PAH & Polycyclic aromatic hydrocarbon \\
\hline PCB & Polychlorinated biphenyl \\
\hline PCR & Polymerase chain Reaction \\
\hline $\operatorname{Pgkl}$ & Phosphoglycerate kinase 1 \\
\hline PhIP & 2-amino-1-methyl-6-phenylimidazo(4,5-b)pyridine \\
\hline
\end{tabular}


PI3K

Ppap2a

Ppia

Ppib

PR-A

PR-B

PyV mT

QPCR

QTL

$\mathrm{R}$

RF

RIN

Rplp2

RR

S

SANT

$\mathrm{SD}$

SERM

SETD 9

Slc38a9

SNP

snRNA
Phosphatidylinositol 3' kinase

Phosphatidic acid phosphatase type 2A

Peptidylprolyl isomerase A (Cyclophilin A)

Peptidylprolyl isomerase B (Cyclophilin B)

Progesterone receptor A

Progesterone receptor B

Polyoma virus middle $T$ antigen

Quantitative polymerase chain reaction

Quantitative trait locus

Resistant mammary carcinoma phenotype

Radiofrequency/microwave radiowave emissions

RNA integrity number

Ribosomal protein, large, P2

Relative Risk

Susceptible mammary carcinoma phenotype

Swi3, Ada2, N-Cor, and TFIIIB domain

Standard deviation

Selective Estrogen Receptor Modulator

SET domain containing 9

Solute carrier family 38 , member 9

Single Nucleotide Polymorphism

Small nuclear RNA 


\section{SS}

STAR

$T b p$

TEB

tet

Tfrc

$\mathrm{U}$

U6

$U b c$

UTR

$\mathrm{WF} / \mathrm{Hsd}$

(WF X COP) F1

WHI

WKy

WMG

Ywhaz
Dahl/Salt-Sensitive rat strain

Specific Targeted Research Project

TATA box binding protein

Terminal end bud

Tetracycline

Transferrin receptor ( $\mathrm{p} 90, \mathrm{CD} 71)$

Undetermined mammary carcinoma phenotype

Spliceosomal small non-coding nuclear RNA component

Ubiquitin C

Untranslated region

Wistar Furth (Harlan Sprague Dawley) rat strain

First generation from a Wistar Furth/Copenhagen mating

Women's Health Initiative

Wistar Kyoto rat strain

Whole mammary gland transplant

Tyrosine 3-monooxygenase/tryptophan 5-monooxygenase activation protein, zeta polypeptide 


\title{
CURRICULUM VITAE
}

\author{
Aaron denDekker \\ University of Louisville School of Medicine \\ Department of Biochemistry and Molecular Biology \\ Louisville, Kentucky 40201 \\ 502-852-7798 \\ Addend01@exchange.louisville.edu
}

\section{EDUCATION}

University of Louisville, Louisville, KY

Ph.D. in Biochemistry and Molecular Biology

2013

Mentor: David J. Samuelson, PhD

Dissertation Title: "Mapping and Characterization of Rat Mammary

Carcinoma Susceptibility QTL Mcs $1 b$ "

University of Louisville, Louisville, KY

M.S. in Biochemistry and Molecular Biology

University of Louisville, Louisville, KY

B.S. in Chemistry

Area of Concentration: Biochemistry

\section{RESEARCH EXPERIENCE}

University of Louisville, Department of Biochemistry and Molecular Biology

Predoctoral Research with David Samuelson, PhD

2008-2012

Dissertation Project: Genetic Mapping and Mechanism of Action of Rat QTL $M c s I b$ 
Research Focus: My research focused on studying a quantitative trait locus in a congenic rat model that associates with an increased susceptibility to developing mammary carcinomas. This locus is identified as mammary carcinoma susceptibility locus $\underline{1 \mathrm{~b}}$, or $M c s 1 b$. This work was composed of three independent parts that focused on 1) fine-mapping the interval of the $M c s 1 b$ QTL on chromosome 2 using congenic rat lines containing a resistant $M c s 1 b$ allele on a susceptible background, 2) identifying whether the $M c s 1 b$-conferred susceptibility phenotype is intrinsic to the mammary gland, and 3) developing a transcript map of genes lying in the locus by sequencing and analyzing gene expression profiles.

Methods/Techniques: Standard molecular biology techniques (i.e. DNA / RNA isolation from tissues and cells, PCR, western blots, northern blots, etc.), cloning, Sanger DNA sequencing, flow cytometry, histology, RT-PCR, gene expression assays, primary cell isolation and culture, radiolabeling, genotyping, animal usage and handling, survival and non-survival surgical techniques.

University of Louisville, Department of Biochemistry and Molecular Biology

Research Technologist in the Zebrafish Mapping Facility in the lab of Ronald Gregg, PhD

2006-2008

Research Focus: My research focused on mapping and cloning mutations in Zebrafish in collaboration with outside investigators. Single point mutations were induced using N-ethyl-N-nitrosurea (ENU) and phenotypes were screened by collaborators. Selected mutations were mapped by our group using microsatellite and single nucleotide polymorphism (SNP) genotyping markers. Once intervals were fine mapped, gene candidates were obtained, cloned and sequenced to identify specific mutations leading to the phenotype.

Methods/Techniques: Positional mapping, PCR, microsatellite and SNP genotyping, cloning, DNA / RNA isolation, Sanger DNA sequencing, pyrosequencing, allelic discrimination assays (real time-PCR based).

University of Louisville, Department of Microbiology and Immunology Research Technologist in the lab of Jill Suttles, PhD

2002-2004

Research Focus: My research focused on two separate projects studying fatty-acid binding proteins (FABPs) and inflammation. The first project focused on the FABP adipocyte protein $2(\mathrm{aP} 2)$ and a role in cholesterol trafficking and inflammatory activity of macrophages. The second project focused on macrophage FABPs and a role in the onset and progression of murine experimental auto-immune encephalitis (EAE), a mouse model of multiple sclerosis.

Methods/Techniques: Cell culture, primary cell isolation, animal usage and handling, murine bone marrow isolation, mouse brain and spinal cord microdissection, histology, immunohistochemistry, ELISAs, flow cytometry, western blots, northern blots, arginase assays, cell proliferation assays. 
University of Louisville, Department of Ophthalmology

Research Technologist in the lab of Maureen McCall, PhD

2001-2002

Research Focus: My work focused on a project studying the gamma aminobutyric acid $\mathrm{C}(\mathrm{GABA}-\mathrm{C})$ receptor in the retina and its role in excitatory responses in vision.

Methods/Techniques: Animal usage and handling, mouse retina and brain microdissection, histology, immunohistochemistry, PCR, genotyping, electroretinograms, DNA / RNA isolation, northern blots.

University of Louisville, Department of Biochemistry and Molecular

Biology

Undergraduate Research project in the lab of Thomas Wheeler, PhD

Research Focus: This undergraduate project focused on studying the ability of fructose-1, 6-bisphosphate (FBP) to permeate artificial multilamellar vesicles as a model for uptake in cardiomyocytes.

Methods/Techniques: Liposome preparation from phosphatidyl choline, radiolabeled-FBP uptake assays, enzymatic assays to measure inorganic phosphate.

\section{RELATED EXPERIENCE}

Baptist Hospital East, Louisville KY

Histotechnologist

2004-2006

Duties: Assist pathologist with gross anatomy of surgical and dermatological specimens, basic histology of specimens, i.e. process, embed, cut and stain, provide general support for the clinical histology laboratory.

Jewish Hospital, Louisville KY

Histotechnologist

2004-2006

Duties were the same as described above.

AWARDS

- Homberger Scholarship

- NIEHS T-32 Predoctoral Training Fellowship 2012 Title: UofL Environmental Health Sciences Training Program PI/Project Leader: David W. Hein, PhD

- Integrated Program in Biological Sciences (IPIBS) Fellowship

2008-2010 


\section{LEADERSHIP}

- Biochemistry and Molecular Biology Student President

2009-2010

- Biochemistry and Molecular Biology Student Vice-President

2010-2011

Graduate Executive Committee Representative

2009-2010

- Training of Undergraduate Summer Students

2009-2011

\section{PUBLICATIONS}

denDekker AD, Xu X, Vaughn MD, Puckett AH, Gardner LL, Lambring CJ, Deschenes L, Samuelson DJ. Rat $M c s l b$ is concordant to the genome wide association identified breast cancer locus at human $5 q 11.2$ and Mier 3 is a candidate cancer susceptibility gene. Cancer Research 2012; 72(22):6002-12. PMID: 22993404

Thomas JL, Vihtelic TS, denDekker AD, Willer G, Luo X, Murphy TR, Gregg RG, Hyde DR, Thummel R. The loss of vacuolar protein sorting 11 (vps11) causes retinal pathogenesis in a vertebrate model of syndromic albinism. Invest Ophthalmol Vis Sci. 2011; 52(6):3119-28. PMID: 21622697.

Wheeler TJ, McCurdy JM, denDekker AD, Chien S. Permeability of fructose-1,6bisphosphate in liposomes and cardiac myocytes. Mol Cell Biochem. 2004; 259(12):105-14. PMID: 15124914

\section{INVITED TALKS}

Cold Spring Harbor, NY

Rat Genomics and Models Meeting, 2009

Title: Rat $M c s I b$ is a mammary gland autonomous QTL and an ortholog of a breast cancer susceptibility locus on human chromosome $5 \mathrm{q}$.

\section{ABSTRACTS PRESENTED}

Samuelson DJ, Xu X, denDekker AD, Sanders J, Kemper AF, Kalbfleisch T. Congenic Mapping and Functional Characterization of a Rat Mammary Carcinoma Susceptibility QTL, $M c s 1 b$, Identifies $M A P 3 K 1$ and MIER3 as Candidate Breast Cancer Susceptibility Genes. Quantitative Genetics \& Genomics Gordon Research Conference, 2011.

Samuelson DJ, Xu X, denDekker AD, Sanders J, Vaughn MD, Lambring CJ, Puckett AH. Comparative Environmental Genomics of Common Breast Cancer Susceptibility Alleles. NIH/NIEHS Center Director's Meeting, 2010. 
denDekker AD, Puckett AH, Vaughn MD, Lambring CJ, and Samuelson DJ. Rat $M c s I b$ is a mammary gland autonomous QTL and an ortholog of a breast cancer susceptibility locus on human chromosome 5q. Rat Genomics \& Models Annual Meeting, Cold Spring Harbor Laboratory, 2009.

Samuelson DJ, denDekker AD, and Puckett AH. Rat Mcs $1 b$ is a comparative genetic model of a human breast cancer susceptibility locus. Poster Presentation, - Annual NIEHS P30 Directors' Meeting, University of Pennsylvania, Philadelphia, 2008. 